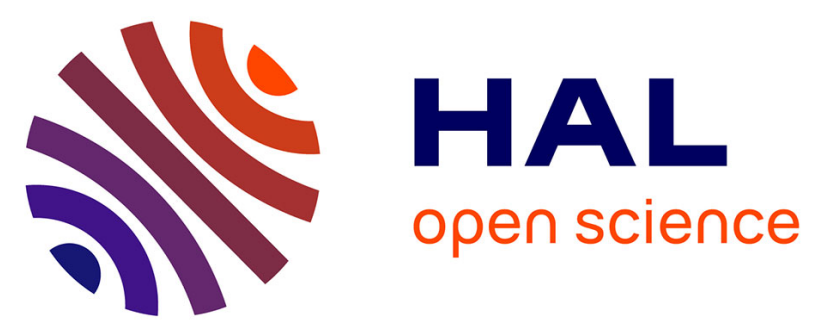

\title{
L'habitat campaniforme de La Noue à Saint-Marcel (Saône-et-Loire) : éléments de définition du groupe bourguignon-jurassien
}

Laure Salanova, Franck Ducreux, Alain Argant, Fabien Convertini, Odette Gros, André-Charles Gros, Sylvie Saintot, Martine Gardini, Françoise Jeudy, Christine Oberlin, et al.

\section{To cite this version:}

Laure Salanova, Franck Ducreux, Alain Argant, Fabien Convertini, Odette Gros, et al.. L'habitat campaniforme de La Noue à Saint-Marcel (Saône-et-Loire): éléments de définition du groupe bourguignon-jurassien. Gallia Préhistoire - Archéologie de la France préhistorique, 2005, 47, pp.33146. 10.3406/galip.2005.2047 . hal-01313071

\section{HAL Id: hal-01313071 \\ https://hal.science/hal-01313071}

Submitted on 21 Jan 2020

HAL is a multi-disciplinary open access archive for the deposit and dissemination of scientific research documents, whether they are published or not. The documents may come from teaching and research institutions in France or abroad, or from public or private research centers.
L'archive ouverte pluridisciplinaire HAL, est destinée au dépôt et à la diffusion de documents scientifiques de niveau recherche, publiés ou non, émanant des établissements d'enseignement et de recherche français ou étrangers, des laboratoires publics ou privés.

\section{(ㅇ)(1) $\$$}

Distributed under a Creative Commons Attribution - NonCommercial - NoDerivatives| 4.0 


\title{
L'HABITAT CAMPANIFORME DE LA NOUE À SAINT-MARCEL (SAÔNE-ET-LOIRE)
}

\section{Éléments de définition du groupe bourguignon-jurassien}

\author{
Sous la direction de Laure SALANOVA et Franck Ducreux \\ Alain Argant, Fabien Convertini, Odette et André-Charles Gros, Sylvie SAIntot \\ Avec la collaboration de Martine GARDInI, Françoise JEUdY, \\ Christine ObERLIN et Yvan VirlogeuX
}

\author{
Introduction (p. 33-36) Franck Ducrevx \\ Présentation du site (p. 36-5l) \\ Localisation, Franck DuCREux \\ Le contexte géologique, Franck Ducrecx \\ La structuration de l'habitat, Franck DucreuX \\ Les restes de faune, Alain ArGanT \\ Datations absolues, Christine OberLin \\ Analyse du mobilier (p. 51-111) \\ La céramique \\ Composition et répartition du corpus, Laure SAIANOVA \\ Caractéristiques techniques des vases, Laure SALANOvA \\ Typologie des formes, Laure SAIANOva \\ Modification des surfaces : décors en creux et éléments en relief, Laure SAIANOVA \\ Analyse spatiale, Laure SAIANOvA \\ Les matières premières et les inclusions d'origine anthropique, Fabien CONVERTINI \\ Composantes culturelles des industries siliceuses taillées, Sylvie SAINTơ \\ Bilan sur l'organisation du mobilier sur le site, Fabien Convertini, Sylvie Saintot et Laure Salanova \\ Le site de La Noue dans le contexte régional et extra-régional (p. 111-141) \\ Le Campaniforme en Bourgogne orientale \\ Historique des recherches, Franck Ducrfux \\ Le Campaniforme en Saône-et-Loire et dans le Chalonnais, Franck Ducreux, Odette et André-Charles Gros \\ Le Campaniforme dans le Dijonnais, Franck Ducreux, Odette et André-Charles Gros, Françoise JEudy \\ et Yvan ViRLogeux \\ Étude des séries de comparaison, Fabien Convertini et Laure Salanova \\ La place de La Noue au sein du Campaniforme bourguignon, Laure Salanova et Sylvie SAINTOT \\ Éléments de définition du groupe bourguignon-jurassien \\ Historique des recherches, Laure SALANOva \\ Approvisionnement en matières premières, Sylvie SaInTOT et Fabien Convertini \\ Le mobilier, Laure SAlanova et Sylvie SAINTOT \\ Les architectures et l'organisation des sites, Franck Ducreux \\ Datations absolues, Laure SAI.ANOVA \\ Caractéristiques du groupe bourguignon-jurassien, Laure SALANOVA \\ Conclusion (p. 141-142) COLLECTIF
}


Alain ARGANT, CNRS, UMR 6636, Institut Dolomieu, 15 rue Maurice-Gignoux, F-38031 Grenoble Cedex. Fabien Convertini, INRAP Méditerranée/UMR 6636 (MMSH/ESEP Aix-en-Provence), 54 avenue du Pont-Juvénal, F-34000 Montpellier.

Franck Ducrfux, INRAP Bourgogne, ZAE de la route du Bassin, Rue A. Berge, F-21800 Sennecey-les-Dijon. Martine GardinI, 34 avenue de l'Europe, F-94190 Villeneuve-Saint-Georges.

André-Charles Gros, L’Oustaou, 91 rue Lazare-Durif, F-07170 Villeneuve-de-Berg.

Françoise JEuDY, INRAP Bourgogne, ZAE de la route du Bassin, Rue A. Berge, F-21800 Sennecey-les-Dijon.

Christine OBERIIN, CNRS, UMR5138, Université Claude-Bernard - Lyon-II, 40 boulevard Niels-Bohr,

F-69622 Villeurbanne Cedex.

Sylvie SAINTOT, INRAP, 12 rue Maggiorini, F-69500 Bron.

Laure SAIANOVA, CNRS, UMR 7041, Protohistoire européenne, Maison de l'archéologie et de l'ethnologie, 21 allée de l'Université, F-92023 Nanterre Cedex.

Yvan Viri.oceux, INRAP Bourgogne, ZAE de la route du Bassin, Rue A. Berge, F-21800 Sennecey-les-Dijon.

Mots-clés. Campaniforme, Bourgogne, habitat, céramique, groupe bourguignon-jurassien.

Résumé. L'habitat campaniforme de La Noue, découvert en 1992 à l'occasion de fouilles préventives, renouvelle considérablement nos connaissances sur la période en Bourgogne orientale et, plus généralement, sur le Campaniforme en contexte domestique.

La répartition au sol du mobilier a permis de délimiter plusieurs unités, dont deux habitations fouillées en intégralité et un probable grenier. Mis à part cette dernière structure, bâtie sur poteaux de bois, les habitations semblent construites sur sablières basses, architecture propice au milieu humide dans lequel était implanté le site.

L'habitat a livré un nombre important de céramiques et une petite série lithique, dans laquelle les grattoirs et les pièces esquillées dominent. Le répertoire ornemental des vases décorés, majoritairement imprimés au peigne, correspond au standard bourguignon (lignes horizontales, bandes hachurées, échelles, cheorons). La céramique commune est quant à elle caractérisée par les types les plus fréquents en Europe (jarres lisses ou à cordon préoral), mais aussi par des types connus en Europe centrale (pichets et cruches, coupes polypodes, vase à anse horizontale). Si les matières siliceuses proviennent de l'environnement local, les analyses réalisées sur l'argile montrent qu'un nombre. significatif de vases proviennent de la rive droite de la Saône.

Ia Noue forme avec les sites de Noir Bois (Alle, Suisse), Champ-Vully (Rances, Suisse) et Derrière le Château (Géoureissiat, Ain) un ensemble de référence pour le groupe campaniforme bourguignon-jurassien qu'il est ainsi possible de définir plus précisément. La confrontation des données montre que La Noue se place dans une position intermédiaire entre Noir Bois, fortement soumis aux influences centre-européennes, et Derrière le Château, affecté par des courants méditerranéens.

Key-words. Bell Beaker culture, Burgundy, settlement site, ceramics.

Abstract. The Bell Beaker settlement site of La Noue, discovered in 1992 during rescue excavations, considerably renews knowledge on this period in the eastern part of the Burgundy region, as well contributing to the general question of Bell Beakers in a domestic context. The spatial distribution of archaeological remains revealed several units, including two complete houses and a probable granary. The latter structure was built on wooden uprights, while the houses seem to have been built on horizontal beams, a form of architecture adapted to local damp conditions.

The settlement produced a large quantity of pottery and a small lithic assemblage dominated by end scrapers and splintered pieces. Decoration patterns on vessels, mainly comb-impressed, are typical of the Burgundy region (horizontal lines, hatched bands, ladder motifs, cheorons). Coarse ware is characterized by types widely found in Europe (undecorated jars or jars with a cordon below the rim), as well as by central European types (jugs and flagons, polypod bowls, vessels with horizontal lugs). While flint raw materials were collected locally; clay analyses reveal that a significant number of vessels come from the right bank of the river Saone.

Together with the sites of Noir Bois and Champ-Vully in Switzerland and Derrière le Château in the Ain region, La Noue provides a representative group of finds for the Burgundy-Jura Bell Beaker group. Comparative analysis shous that La Noue occupies an intermediate position between Noir Bois, heavily influenced by central Europe, and Derrière le Château, related more to the Mediterranean.

Translated by M. Illet and M. Vanhaeren 


\begin{abstract}
Schlagwörter. Glockenbecherkultur, Burgund, Siedlung, Keramik.
Zusammenfassung. Die 1992 bei Präventivgrabungen entdeckte Glockenbechersiedlung von La Noue bringt einen erheblichen Wissenszuwachs über die betreffende Epoche in der östlichen Bourgogne und ganz allgemein über Siedlungen dieser Kulturgruppe. Anhand der räumlichen Verteilung des Fundmaterials ließen sich verschiedene Einheiten eingrenzen, darunter zwei vollständig durch die Ausgrabung erfaßte Wohngebäude und ein möglicher Speicherbau. Anders als dieser Pfostenbau sind die Wohngebäude anscheinend Schwellbalkenkonstruktionen. Diese Bauweise bot sich an, da die Siedlung im Feuchtmilieu errichtet war. Der Platz liefert eine große Zahl keramischer Funden und eine kleine Serie von Steingeräten (vorwiegend Kratzer und Abschläge). Der Musterschatz der (überwiegend durch Kammabdruck) verzierten Gefäße (horizontale Linien, strichgefüllte Bänder, Leiter- und Winkelbänder) entspricht dem, was aus der Bourgogne bisher bekannt wurde. Die Keramik läßt sich vorweg durch die in ganz Europa gängigen Formen (glattrandige Gefäße und solche mit Randleiste) charakterisieren, umfaßt aber auch typische zentraleuropäische Formen (Becher und Krüge, Vielfußtassen, Gefäße mit Horizontalhenkel). Während das Silexmaterial aus dem lokalen Umfeld kommt, zeigen die Tonanalysen, daß eine ganze Reihe von Keramikgefäßen vom rechten Ufer der Saône stammen.

Zusammen mit den Fundstellen von Noir Bois (Alle, Suisse), Champ-Vully (Rances, Suisse) und Derrière le Château (Géovreissiat, Ain) bildet die von La Noue eine Referenzserie für die Glockenbechergruppe in Burgund und im Jura, die sich nun auch besser definieren läßt. Im Vergleich zeigt sich, daß La Noue eine Mittlerstellung zuvischen dem stark zentraleuropäischen Einflüssen unterliegenden Noir Bois und dem von mediterranen Kulturströmungen geprägten Derrière le Château einnimmt.
\end{abstract}

Übersetzung: Stefan WIRTH

La fouille et l'étude du gisement de La Noue apportent non seulement des connaissances inédites sur un habitat de plaine attribué dans sa totalité à la culture campaniforme, mais aussi des données de premier ordre sur une période dont les rares témoignages étaient jusqu'alors fondus dans des séries concernant des groupes culturels à caractère local '. Cette découverte conduit à redessiner le paysage culturel et le dynamisme dù val de Saône à l'aube de la Protohistoire. Les nouvelles opérations de sauvetage mises en place depuis, en région dijonnaise notamment, apportent d'importants éléments permettant de compléter une typologie déjà riche et variée.

Le site de La Noue a été mis au jour lors de la phase de reconnaissance archéologique préliminaire aux travaux routiers réalisés sur la commune de Saint-Marcel (Saôneet-Loire) et visant à dévier la $\mathrm{RN} 73$ pour contourner l'agglomération par le nord. Le diagnostic, réalisé en 1992 (Roiné, 1992), avait abouti à la reconnaissance de 5 sites, toutes époques confondues, dispersés sur le tracé global du projet (fig. 1). Les sondages réalisés sur le site de La Noue, à l'extrémité sud du tracé, avaient révélé la présence d'un mobilier erratique, formé principalement de fragments de céramique protohistorique, de datation difficile,

\footnotetext{
1. Pour leur aide précieuse, nous tenons à remercier les membres du sevice régional de l'Archéologie de Bourgogne, en particulier Béatrice Bonnamour. Nous souhaitons également remercier Louis Bonnamour, conservateur du musée de Chalon-sur-Saône, pour le temps qu'il a bien voulu nous consacrer et les informations de premier ordre qu'il nous a communiquées.
}

et de quelques pièces lithiques atypiques. Le seul tesson attribué au Chalcolithique portait une série de lignes horizontales imprimées au peigne, provenant d'un gobelet campaniforme. Comme nous le verrons plus loin, ces sondages ont été réalisés hors des zones de forte concentration de mobilier ou structurées, ne permettant pas une bonne interprétation du site. En revanche, cette situation a permis la bonne conservation des zones archéologiques qui auraient pu être fortement endommagées si des sondages étaient venus les traverser. Afin de préciser la datation de l'ensemble des vestiges et leur éventuelle organisation, une fouille complémentaire du site a été mise en place par le Service archéologique de la région Bourgogne.

L'opération archéologique a été réalisée en novembre et décembre 1995, en simultanéité avec la fouille du site Sur le Bief, à l'extrémité nord-est du projet routier, concernant un petit bâtiment gallo-romain. Le décapage extensif de la totalité de la surface du gisement concernée par les futurs travaux, situé entre les profils 15 et 25 du projet routier (soit $10000 \mathrm{~m}^{2}$ ), a d'emblée révélé la présence de structures excavées et d'un niveau archéologique organisé en zones de fortes concentrations de mobilier. Le ramassage du mobilier hors stratigraphie laissait envisager un ensemble homogène, constitué de tessons décorés appartenant au Campaniforme et d'une céramique plus grossière identifiée comme de la céramique d'accompagnement. Ces vases étaient associés à une industrie lithique peu abondante, de la faune sous forme d'esquilles carbonisées dans le niveau archéologique 

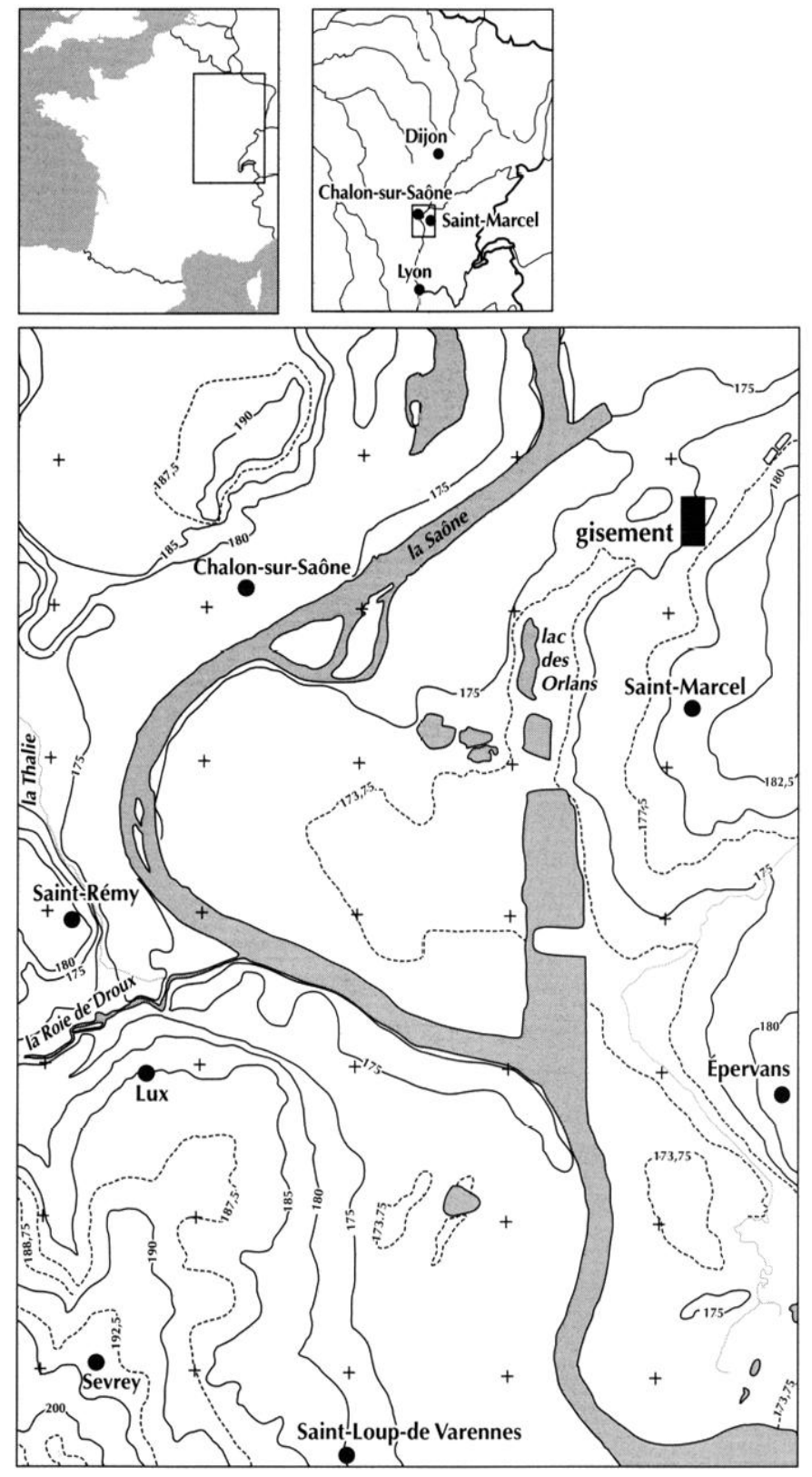

Fig. 1 - Situation géographique du gisement de La Noue (cartes F. Ducreux et Y. Amrane d'après carte IGN 1/25 000, $n^{\prime \prime} 3026$ ).

et sous forme de pièces mieux conservées dans les structures en creux, ainsi qu'à quelques fragments d'argile cuite.

Le niveau archéologique et les structures en creux ont été fouillés en totalité lors de l'opération archéologique, révélant un mobilier abondant, homogène et dans un état de conservation satisfaisant. L'ensemble du matériel archéologique découvert en position stratigraphique a fait l'objet d'un relevé de répartition en abscisses et en ordonnées pour aboutir à un plan d'ensemble permettant une bonne appréhension des zones de concentration. Toutes ces zones ont été soumises à des cotations en vue d'un nivellement général. La fouille a été réalisée de façon plus fine dans les secteurs à forte densité de vestiges tandis que les zones stériles, assez nombreuses, ont été traitées par un nettoyage plus rapide à la rasette. Plusieurs sondages d'une trentaine de centimètres de hauteur ont été réalisés afin de s'assurer de la stratigraphie (Ducreux, Amrane, 1996).

Le site de La Noue est le premier habitat structuré en plaine de Saône à s'associer pleinement au Campaniforme en Bourgogne. Il entre de ce fait en contradiction avec les habitats chalcolithiques connus jusqu'alors, associant toujours quelques rares tessons à décor campaniforme à une céramique de type régional plus abondante comme à Ouroux-sur-Saône (Thévenot et al., 1976), au Breuil à SaintMarcel (Bonnamour, Moyne, 1963) et au Camp à Chassey (Thévenot, 1990).

F. D.

\section{PRÉSENTATION DU SITE}

\section{LOCALISATION}

Le gisement est localisé en Saône-et-Loire entre les agglomérations de Chalon-sur-Saône et de Saint-Marcel, près des lieux-dits La Noue et Les Orlans, à quelques dizaines de mètres seulement au nord-est du lac des Orlans, probable bras fossile de la Saône (fig. 1). La proximité du lit de la Saône et la localisation du site dans une légère dépression, à une hauteur d'environ 174,5 m NGF, font que la zone est fréquemment inondée par les crues actuelles. Après la fouille du site, il est apparu que le niveau archéologique, intercalé entre un lit de graviers et une quarantaine de centimètres de terre végétale, n'a pas été gravement perturbé par ces inondations. Le mobilier de type charbon de bois, présent dans les structures en creux, est en revanche fortement délavé. Cette situation en zone inondable est inhabituelle pour les habitats des périodes néolithiques et chalcolithiques de la région. Si l'on se réfère aux exemples des sites du Breuil à Saint-Marcel, du Moulin de Droux à Lux et de la station du Mont à Crissey (Gros, 1968), il apparaît que ces implantations sont toutes situées au-delà ou en bordure de la cote de $177 \mathrm{~m}$, limite actuelle de la zone soumise aux crues. Hors de la région, cette constatation ne s'applique pas systématiquement aux sites campaniformes et l'on connaît un certain nombre d'exemples qui présentent le même type d'implantation qu'à La Noue (Billard et al., 1991). 
D’après les études géomorphologiques récentes de C. Ferrier, réalisées sur la vallée de la Saône au nord de Chalon-sur-Saône, le lit de la Saône n'aurait pas connu de déplacements majeurs dans la plaine chalonnaise depuis le Néolithique (Argant et al., 1996). La description du site naturel de Chalon-sur-Saône réalisée par B. Trémeau (1984) offre l'hypothèse d'une fréquence nettement moins élevée des crues au cours des périodes néolithiques et protohistoriques qu'au cours des périodes historiques, mais n'admet en aucun cas que la région du lac des Orlans ait été située en zone sèche au Néolithique. De son côté, M. Gardini suggère qu'au III $^{\mathrm{e}}$ millénaire avant J.-C., plusieurs bras devaient être présents, mais rien n'indique que le site de La Noue était localisé en zone inondable (Gardini, 2000) ${ }^{2}$.

Même si les éléments de certitude font défaut, en l'absence d'études paléoenvironnementales précises, le cadre naturel du site de La Noue pose plusieurs questions quant aux motivations ayant poussé une population à s'établir dans un tel milieu. D'emblée, plusieurs hypothèses nous viennent à l'esprit: la méconnaissance du milieu naturel local par une population exogène, un habitat temporaire mis en place en période estivale, un habitat durable en aire ouverte disposant d'une architecture adaptée aux crues.

La fouille intégrale du gisement pourrait apporter des éléments de réponses ${ }^{3}$.

F. D.

\section{LE CONTEXTE GÉOLOGIQUE}

Sur le plan géologique, l'ensemble du gisement se trouve en contexte alluvionnaire, correspondant au lit majeur de la Saône. Ces alluvions se retrouvent sous forme de niveaux de limons sableux séparés par des couches de graviers et de sables. Ce contexte géologique est homogène sur toute la partie décapée du site, malgré quelques irrégularités remarquées au niveau de la couche de graviers (fig. 2). La présence de lits de graviers superposés laisse supposer que le site est installé sur le sommet du comblement d'un ancien lit de la Saône, peut-être le bras fossile présumé des Orlans (fig. 3).

F. D.

2. Dans le cadre du projet de publication du site de La Noue, Martine Gardini s'est rendue sur le site et a ćtudié plusieurs coupes des sondages.

3. Le site n’a pas été fouillé sur sa surface entière, mais uniquement sur les zones menacées par le projet routier.

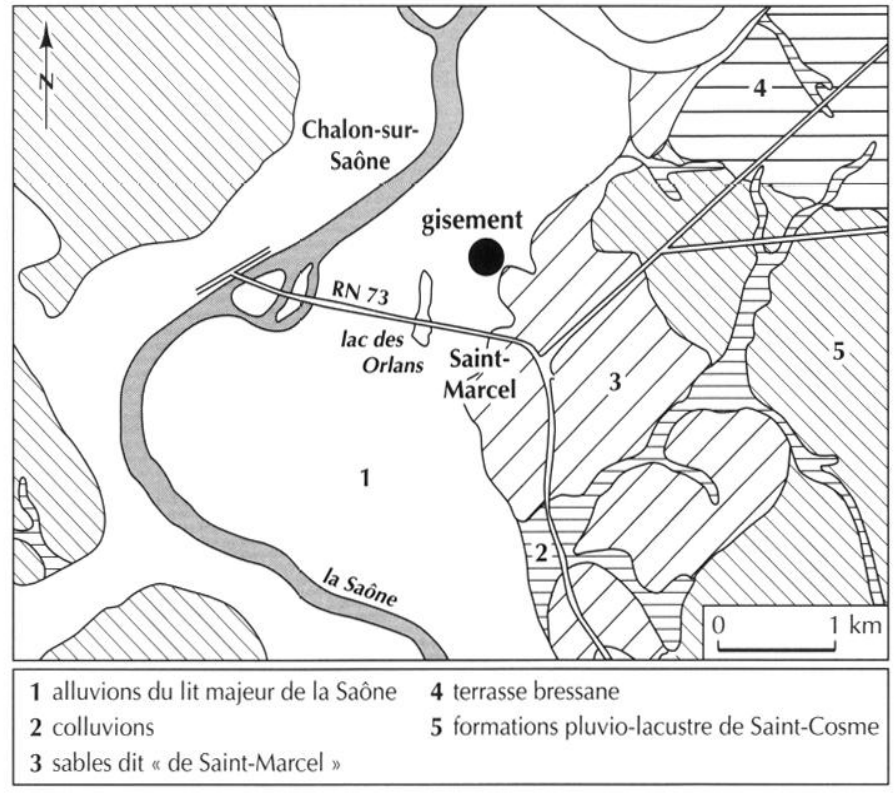

Fig. 2 - Formations géologiques autour de Saint-Marcel (carte F. Ducreux).

\section{LA STRUCTURATION DE L'HABITAT}

\section{LA STRATIGRAPHIE}

Le site se développe sur une surface de $3200 \mathrm{~m}^{2}$ entre les profils 20 et 23 du tracé routier (coordonnées Lambert $\mathrm{X}$ : 794,950, Y: 2201 et $7: 175 \mathrm{~m}$ ). Le niveau archéologique, épais de 2 à $3 \mathrm{~cm}$ au maximum, est implanté sur la dernière couche de graviers formant le sommet du sol géologique alluvionnaire. Il est recouvert par une couche d'une vingtaine de centimètres, formée d'un limon très fin de couleur beige, elle-même recouverte par une épaisseur de $20 \mathrm{~cm}$ environ de terre végétale fortement sableuse. Cette stratigraphie est observée sur l'ensemble du site. Un sondage profond, pratiqué dans une zone dépourvue de traces archéologiques (fig. 3), montre que les couches supérieures de graviers sur lesquelles est installé le sol archéologique ne sont épaisses que d'une trentaine de centimètres et alternent ensuite avec des matériaux sableux (fig. 2). Les formations graveleuses de surface ont sans doute joué le rôle de chape et protégé le sol d'occupation préhistorique de l'altération inévitable qui aurait eu lieu s'il avait été directement posé sur les sables fins. La présence de graviers a peut-être été ici déterminante dans le choix du secteur comme zone d'habitat. Les perturbations du niveau archéologique, visibles en certains endroits, sont par contre dues aux travaux agricoles, notamment la culture du maïs, qui ont engendré de rares sillons profonds. Ces sillons sont 
Coupe géologique

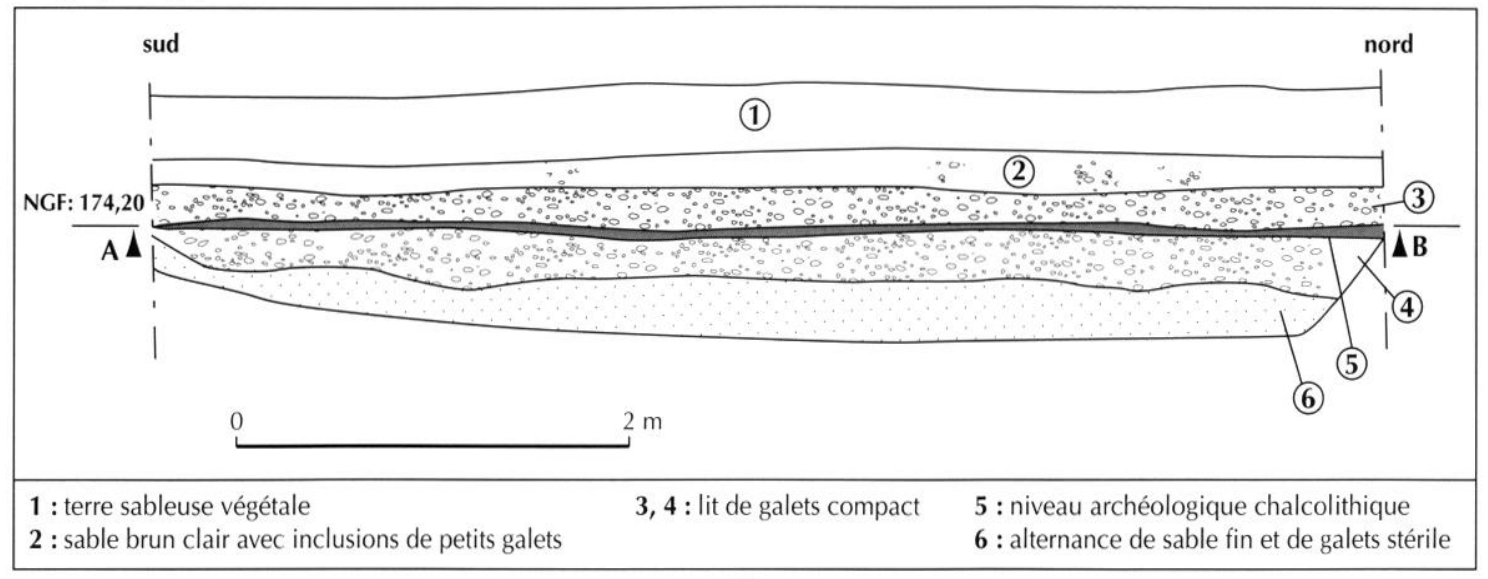

a

Stratigraphie générale schématique

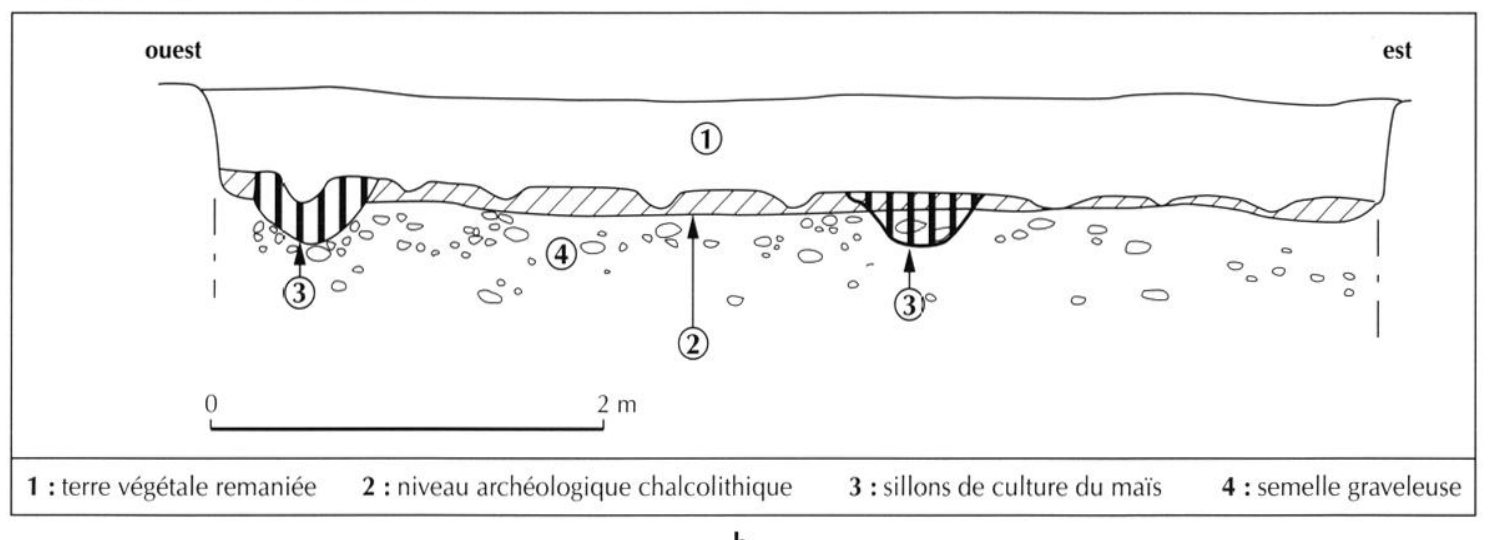

b

Fig. 3 - Formations géologiques du gisement de La Noue (l'implantation des coupes est indiquée fig. 4). Levé F. Ducreux.

comblés par une terre limoneuse brune contenant un mobilier protohistorique et gallo-romain peu abondant, caractérisé par des tessons très érodés.

\section{LES ZONES DE CONCENTRATION DE MOBILIER ET LES STRUCTURES EN CREUX}

I.es zones de concentration apparues au décapage se sont pleinement confirmées au cours de la fouille et de la réalisation du relevé général. Ce plan met en évidence cinq zones ou locus à fortes concentrations de mobilier, séparées par des espaces vides, ainsi qu'un sixième secteur situé à l'écart du niveau archéologique, comprenant des structures en creux. Sur ces six secteurs, deux concentrations ont pu être étudiées dans la quasi-totalité de leurs surfaces: elles seront nommées locus 1 et 2 . Les locus 4 et 6 , situés contre la bordure occidentale de la fouille, n'ont pu être étudiés que sur une très faible surface. Leur prolongement au-delà de la limite de fouille laisse supposer une poursuite du gisement vers l'ouest (fig. 4).

\section{Le locus 1}

Cet ensemble comporte une zone de forte concentration de mobilier comprenant des vases visiblement écrasés sur place, des tessons, quelques pièces lithiques et des esquilles osseuses carbonisées et dispersées sur une surface grossièrement rectangulaire de $12 \mathrm{~m}$ de longueur sur $8 \mathrm{~m}$ de largeur, soit $96 \mathrm{~m}^{2}$ (fig. 5). Le niveau repose sur une couche de graviers stable et le mobilier, contenu dans une gangue limoneuse très pure, apparaît dans un état de conservation satisfaisant. Deux fosses, contenant un mobilier archéologique important, s'associent à cette zone (UF 1 et UF 2). 


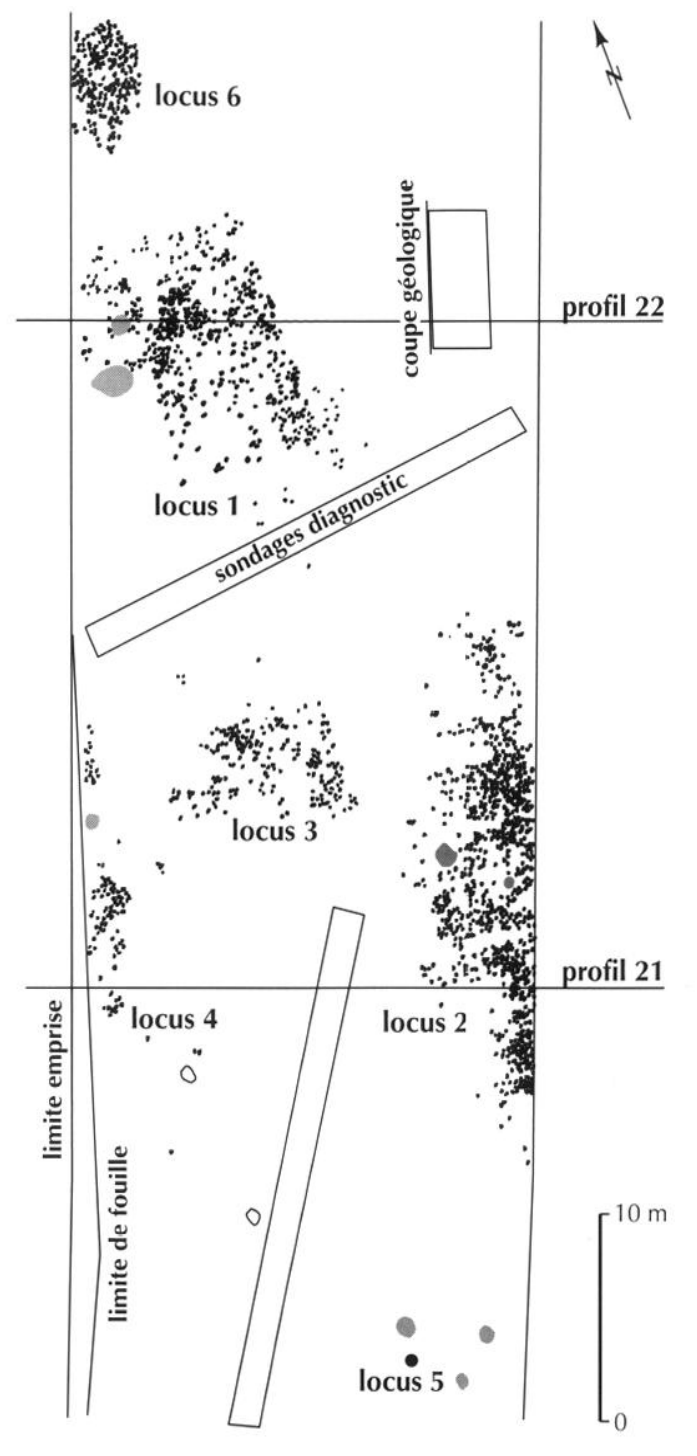

structures en creux pierres calcaires

Fig. 4 - Plan général du niveau archéologique et des structures (relevé F. Ducreux).

\section{La répartition du mobilier}

D'emblée, deux constatations s'imposent à la vue du plan de répartition du mobilier (fig. 5). La première concerne la dispersion irrégulière de la céramique, concentrée sur de faibles surfaces. La fouille a révélé que ces zones de concentration correspondaient aux emplacements de vases écrasés sur place, ce qui laisse supposer une bonne conservation du niveau archéologique. La seconde renvoie aux délimitations rectilignes et orthogonales distinguant les zones à mobilier des zones stériles. Ces délimitations provoquent des effets de parois assez. nets sur les côtés nord, est et ouest du locus. Une fois restitué en plan, cet ensemble définit un rectangle de $9 \mathrm{~m}$ de longueur sur une largeur de $7 \mathrm{~m}$, orienté nord-sud. Les structures en creux sont situées hors de cet espace, mais demeurent à proximité immédiate.

\section{Les structures en creux}

Deux fosses sont présentes sur le côté ouest du locus (UF 1 et UF 2).

La fosse UF l est un creusement de forme irrégulière, grossièrement ovoïde, de $2 \mathrm{~m}$ de longueur sur $1,4 \mathrm{~m}$ de largeur maximum, pour une profondeur de $0,8 \mathrm{~m}$. Son remplissage est formé d'un limon brun à beige, légèrement plus foncé que le substrat encaissant. Le mobilier céramique en rapport avec cette structure est fragmenté.

La fosse UF 2 se présente sous un aspect plus régulier que la précédente. La forme du creusement en surface est circulaire, de $40 \mathrm{~cm}$ de diamètre pour une profondeur de $30 \mathrm{~cm}$. De même que pour la fosse UF l, le remplissage de cette structure est formé d'un limon brun clair assez proche du sol géologique. Les tessons de 2 vases peu fragmentés ont été découverts dans cette fosse.

\section{Le locus 2}

Le locus 2 se situe dans la partie sud-est du site et occupe une surface de $24 \mathrm{~m}$ de longueur sur $8 \mathrm{~m}$ de largeur, soit $192 \mathrm{~m}^{2}$ (fig. 6). L'ensemble fouillé n'est pas complet puisque le niveau archéologique se prolonge vers le sud-est, hors de la limite de fouille. Le niveau archéologique se situe ici sur une couche de graviers régulière, plus épaisse que celle du locus 1 et apparaît entre les cotes NGF de 174,4 m et de 174,5 m. Ce niveau a livré un mobilier très abondant, surtout dans la partie située près de la limite est de la fouille.

\section{La répartition du mobilier}

Le mobilier se répartit plus équitablement que dans le locus 1 et, contrairement au locus 1 , des vases écrasés en place n'ont pas été identifiés. Toutefois, la présence de tessons appartenant à un même vase et relevés à proximité les uns des autres peut indiquer une structuration de l'espace semblable à celle du locus 1. Cette observation suggère une altération légèrement plus forte pour cet espace que pour le locus 1 . Le locus 2 dispose néanmoins de conditions de conservation suffisantes pour en permettre une étude spatiale. De même que pour le locus l, le plan de répartition du mobilier fait apparaitre plusieurs axes orthogonaux qui séparent des zones à forte proportion de 


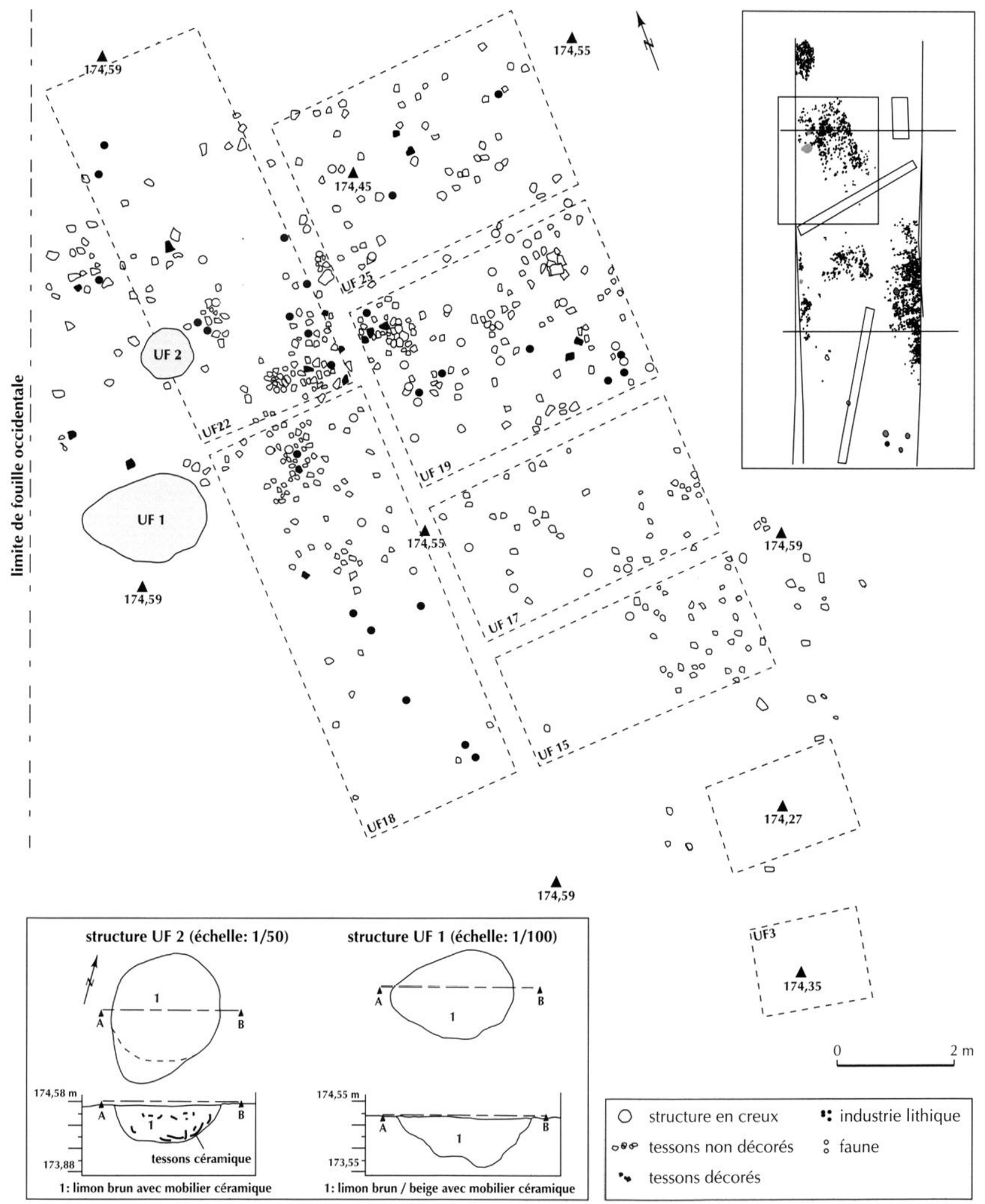

Fig. 5 - Plan général du locus 1 (relevé F. Ducreux). 

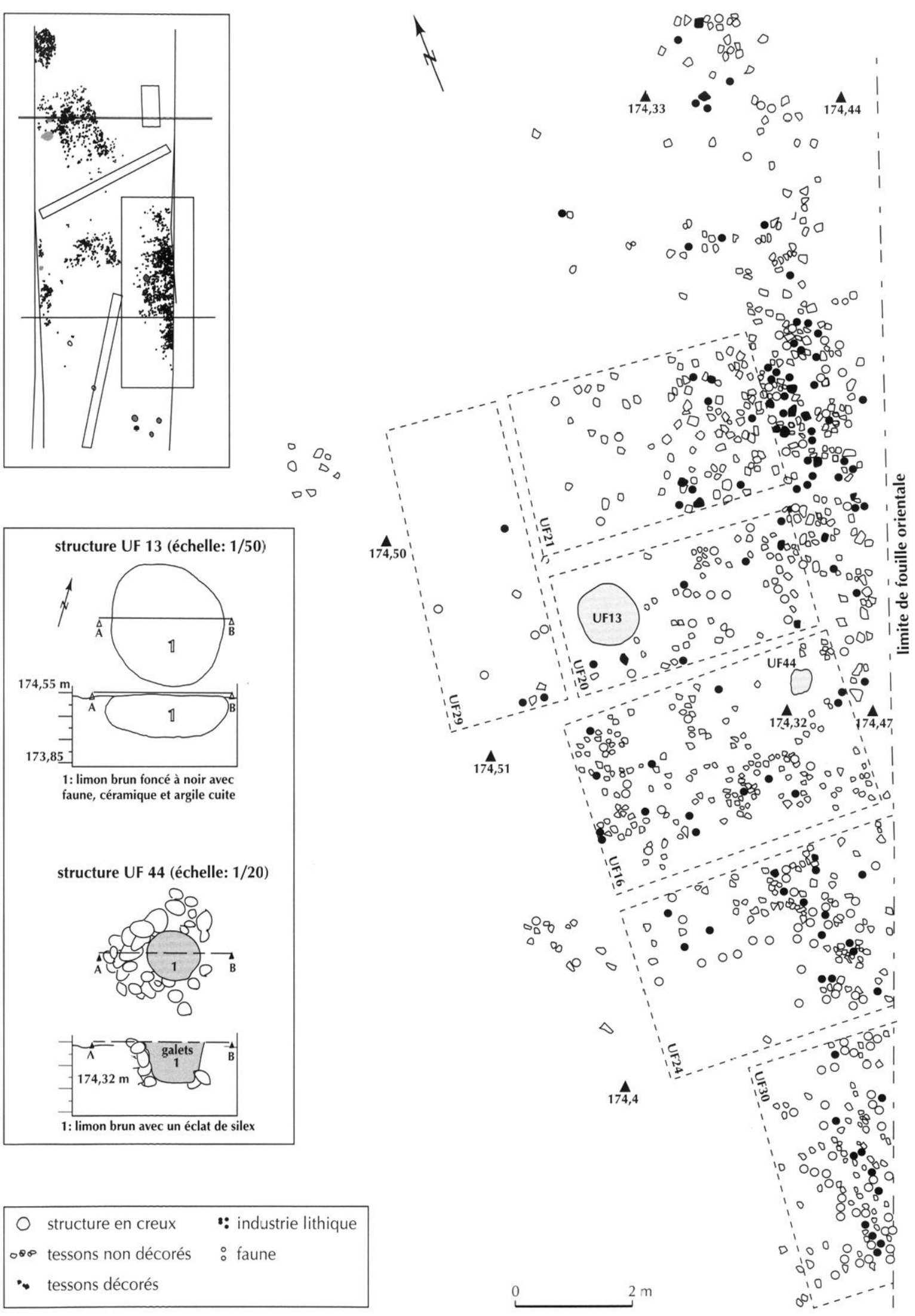

Fig. 6 - Plan général du locus 2 (relevé F. Ducreux). 

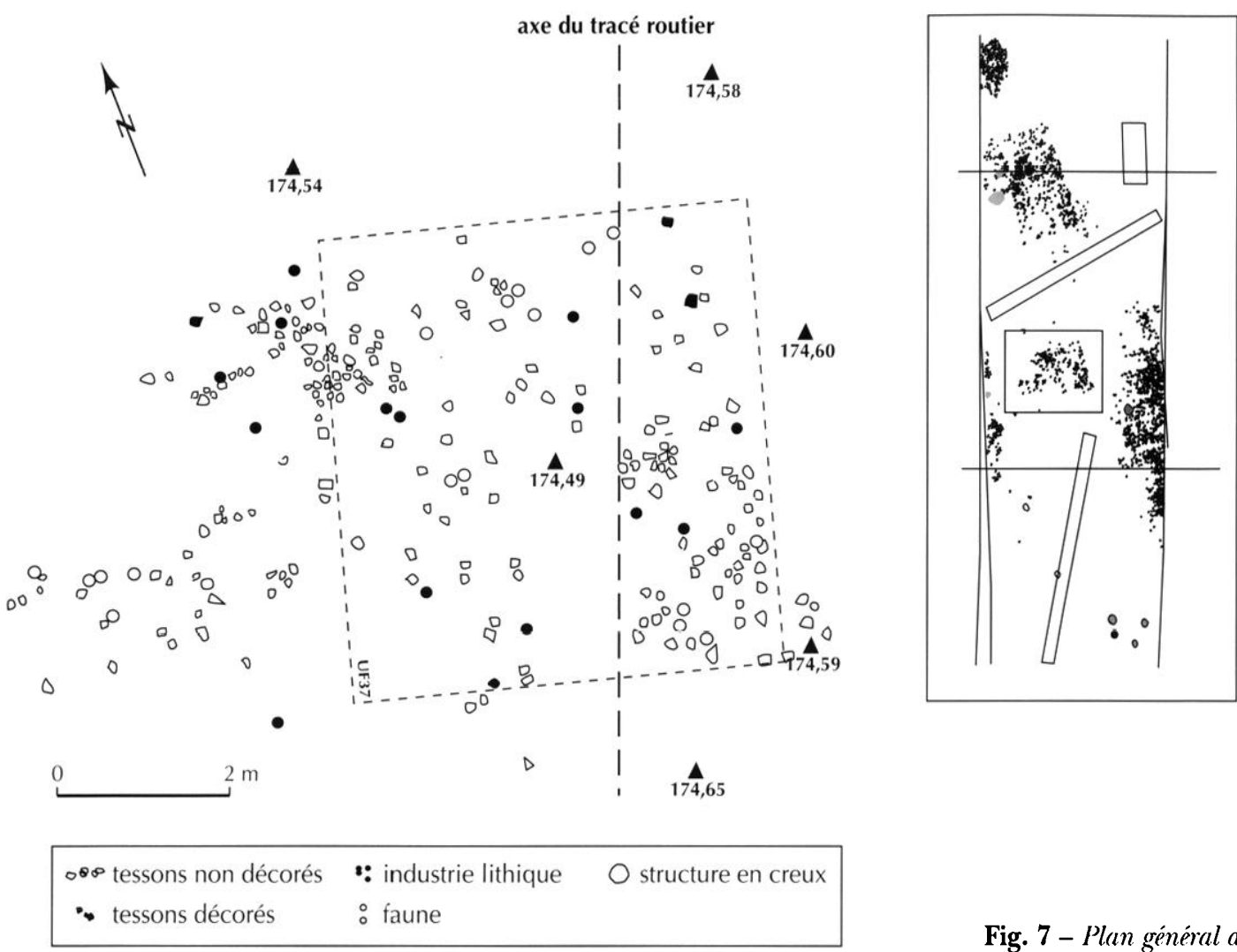

Fig. 7 - Plan général du locus 3 (relevé F. Ducreux).

mobilier de zones stériles. Ces effets de parois forment un rectangle de $10 \mathrm{~m}$ de longueur sur $7 \mathrm{~m}$ de largeur, orienté nord-sud, dans lequel sont situées les structures UF 13 et UF 44. D'autres axes, d'orientation semblable, se remarquent au nord et au sud, suggérant une organisation spatiale identique à celle de l'espace central.

\section{Les structures en creux}

La fosse UF 13 est un creusement de plan circulaire et régulier d'un diamètre de $90 \mathrm{~cm}$ à l'ouverture pour une profondeur de $35 \mathrm{~cm}$. Les parois de la fosse sont en forme de cloche, à la manière des silos protohistoriques, de telle sorte que le diamètre du fond de la structure est supérieur de $10 \mathrm{~cm}$ au diamètre d'ouverture. La faible profondeur du creusement nous empêche toutefois d'assimiler cette structure de façon sûre à un silo. Le remplissage est formé d'un limon brun foncé à gris qui se démarque assez nettement du sol encaissant. Le mobilier recueilli est formé pour l'essentiel de faune et de quelques tessons. La fonction finale de ce creusement est de façon évidente celle d'un dépotoir domestique.

La fosse UF 44, qui se démarque du sol géologique par un creusement de $20 \mathrm{~cm}$ de diamètre, est délimitée par une couronne de galets de module supérieur à celui des galets présents dans le substrat géologique du secteur. Le creusement est de plan circulaire, d'une profondeur de $12 \mathrm{~cm}$. Il présente des parois obliques et un fond régulier. Le remplissage de la structure est formé d'un limon très proche du substrat géologique et contient un éclat de silex. La couronne de galets peut s'apparenter à un calage de poteau. En raison des difficultés de lecture, le fond de la structure n'a pas forcément été atteint. L'hypothèse du calage d'un vase de stockage peut également être retenue.

\section{Le locus 3}

Le locus 3 se situe au centre du gisement et se trouve traversé par l'axe du projet routier, entre les profils 21 et 22 (fig. 7). Il se matérialise par un niveau archéologique, situé entre les cotes de $174,5 \mathrm{~m}$ et $174,65 \mathrm{~m}$, composé de tessons assez fragmentés pour l'essentiel. Les tessons sont posés sur une couche géologique où se mêlent graviers et limon. Il est aussi possible de remarquer la présence d'esquilles osseuses carbonisées et de quelques éclats de silex dispersés sur l'ensemble de la zone de répartition qui n'occupe qu'une quarantaine de mètres carrés. En comparaison avec les locus 1 

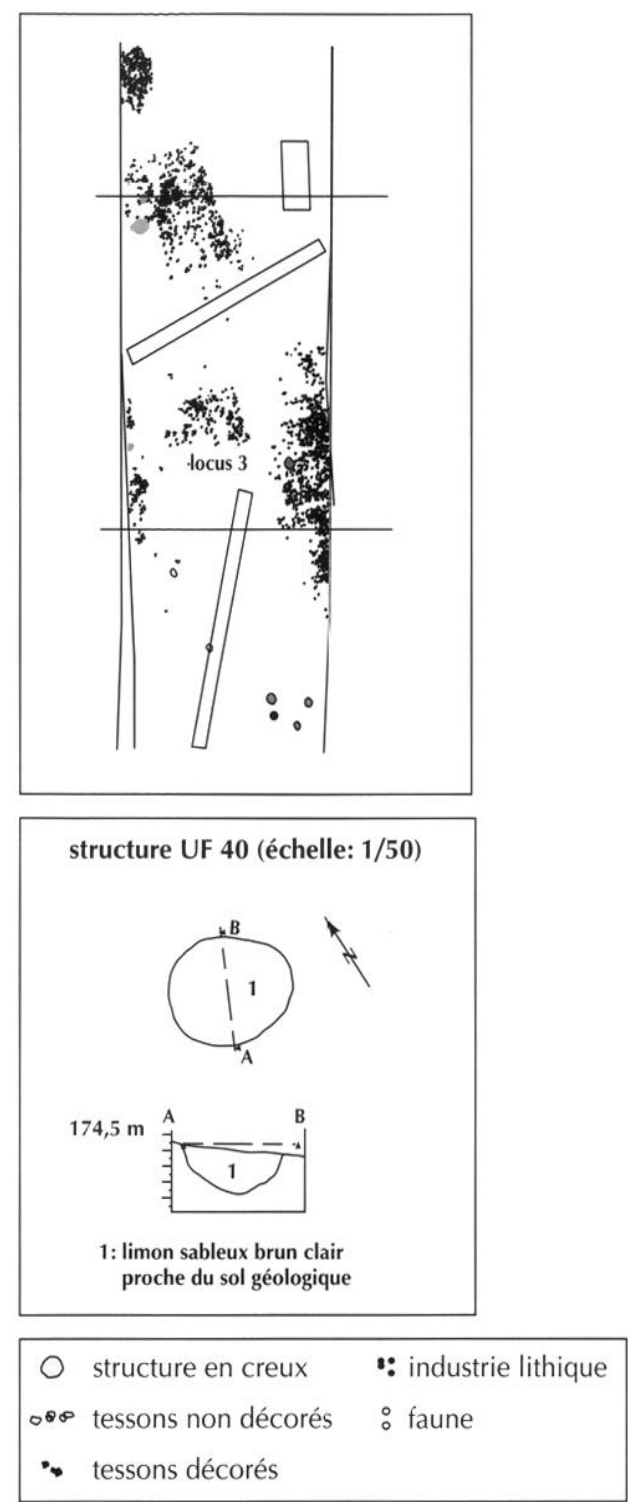

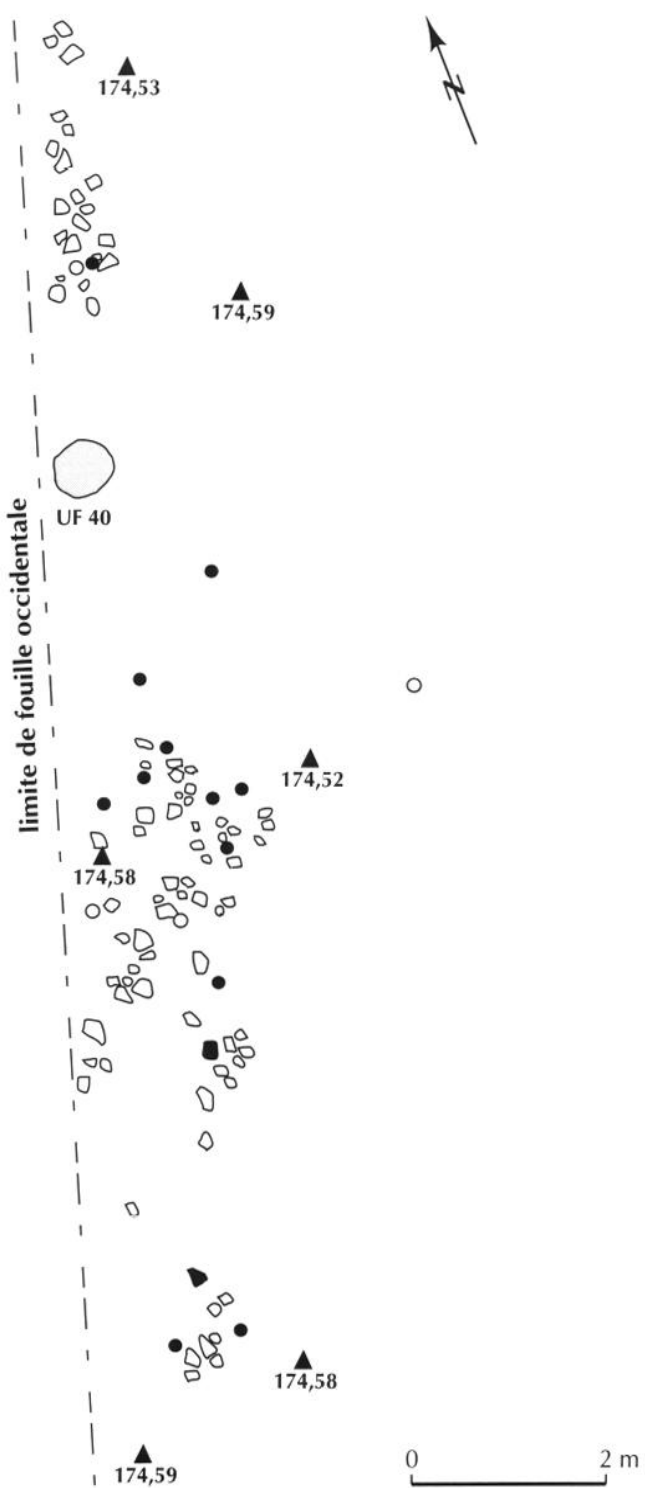

Fig. 8 - Plan général du locus 4 (relevé F. Ducreux).

et 2 , cette surface est faible et cette remarque nous incite à penser que nous nous trouvons cn présence d'un lambeau de couche archéologique ayant appartenu à une unité plus vaste. Cette hypothèse se trouve confirmée par le nivellement du secteur qui indique que la couche archéologique est déposée sur une zone légèrement plus haute que celle des autres locus.

De même que pour les deux locus précédents, deux axes, remarquables dans la répartition du mobilier, forment un angle à $90^{\circ}$ dans le coin nord-est du locus. Ces axes présentent la même orientation que celle des locus 1 et 2 .

\section{Le locus 4}

Le locus 4 est situé contre la limite occidentale de la fouille. Il n'a pu être appréhendé que sur une très faible surface (fig. 8). Le mobilier se répartit de façon régulière, au nord et au sud du locus. Une zone de moindre concentration est cependant remarquable au centre du locus, à la périphérie d'une structure en creux de type trou de poteau. Parmi le mobilier céramique, nous pouvons signaler la présence de plusieurs éléments appartenant à un récipient de type faisselle, apparemment écrasé sur place. La faune et 


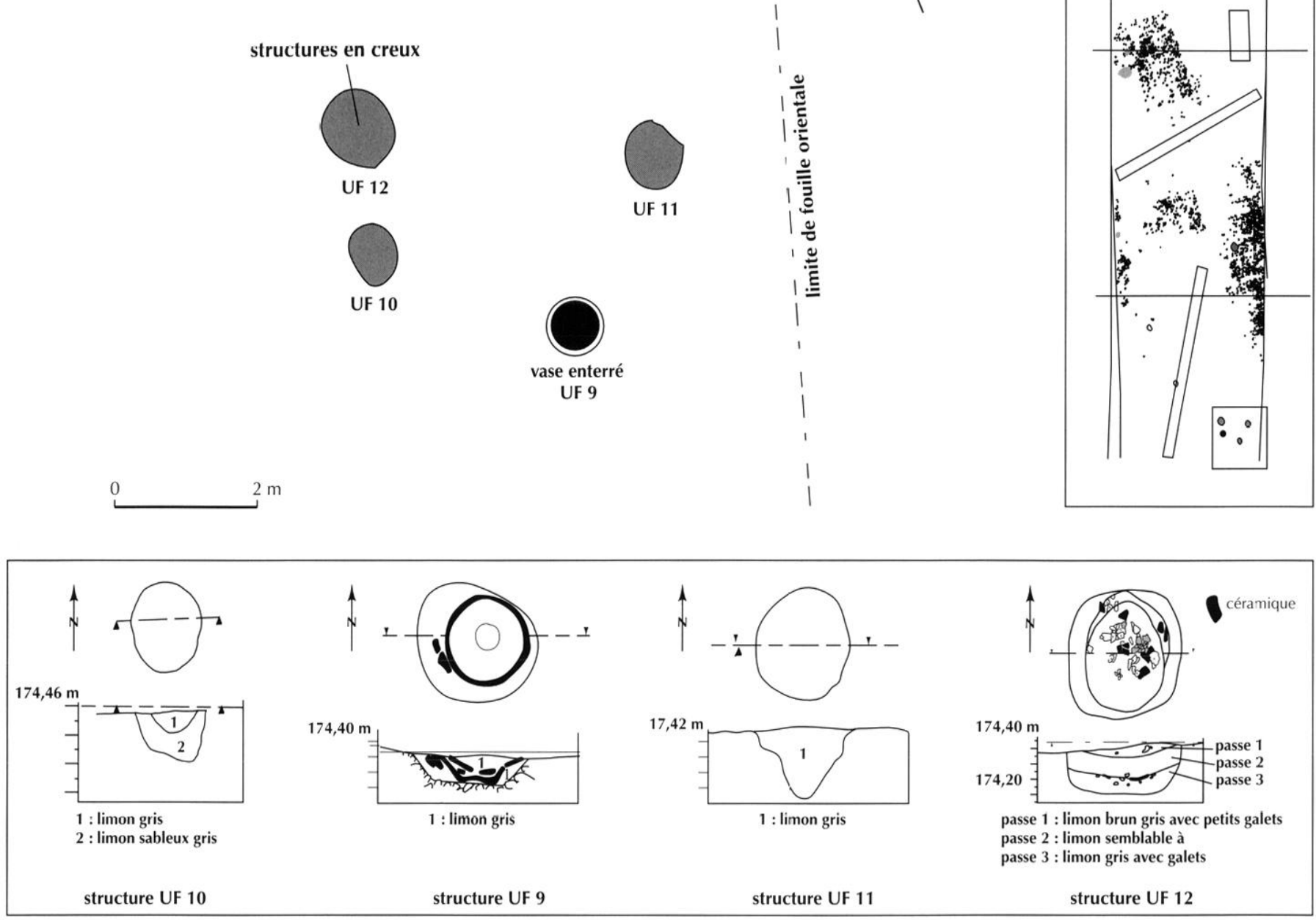

Fig. 9 - Plan général du locus 5 (relevé E. Ducreux).

l'industrie lithique sont essentiellement dispersées dans la partie sud du locus.

Un effet de paroi, orienté selon un axe nord/sud, est pressenti au niveau de la limite est du locus.

\section{Les structures en creux}

La fosse UF 40 est située au nord du locus, près de la limite de fouille occidentale. Il s'agit d'un creusement circulaire de $50 \mathrm{~cm}$ de diamètre pour une profondeur de $20 \mathrm{~cm}$. Le remplissage de ce creusement, proche du sédiment constituant le sol géologique, ne contient aucun mobilier. Ce type de structure reste difficile à interpréter.

\section{Le locus 5}

Ce locus est excentré par rapport aux locus précédents. Il présente également une organisation tout à fait différentc (fig. 9); aucun niveau de mobilier n'est en effet visible. Quatre structures en creux (UF 9, UF 10, UF 11 et UF 12) se rencontrent sur une surface relativement restreinte de $30 \mathrm{~m}^{2}$.

La structure UF 9 est constituée par le fond d'un vase d'une trentaine de centimètres de diamètre, enterré et conservé seulement sur une vingtaine de centimètres de hauteur. Le bord du vase à été retrouvé à l'intérieur de la structure, laissant suggérer une bonne conservation des 
vestiges dans cette zone. Ce type de structure est classique du Néolithique jusqu'à la fin de la Protohistoire et s'associe en règle générale au stockage de denrées alimentaires.

La structure UF 10 est un creusement circulaire irrégulier de $50 \mathrm{~cm}$ de diamètre, pour une profondeur de $35 \mathrm{~cm}$. Son remplissage est formé d'un limon sableux de couleur grise, tranchant nettement avec le substrat géologique. Ce sédiment ne contient que quelques éclats de silex.

La structure UF 11 est, quant à elle, matérialisée par un creusement circulaire de $65 \mathrm{~cm}$ de diamètre pour une profondeur de $40 \mathrm{~cm}$. Le creusement adopte un profil en " $\mathrm{V}$ " assez typique des trous de poteau. Le remplissage, semblable à celui de la structure UF 10, ne contenait qu'un éclat de silex. La morphologie du creusement de la structure peut inciter à interpréter cette structure comme un trou de poteau, ceci malgré un diamètre important.

Enfin, la structure UF 12 est un creusement circulaire de $80 \mathrm{~cm}$ de diamètre, pour une profondeur de $32 \mathrm{~cm}$. Les parois et le fond du creusement sont très réguliers. Le comblement est constitué d'une superposition de trois couches:

- couche 1: comblement final de la fosse, limon sableux gris à brun foncé, contenant des graviers;

- couche 2 : limon sableux semblable à celui de la couche 1 , mais plus clair;

- couche 3: limon sableux gris contenant quelques tessons, de la faune sous forme d'esquilles brûlées et une industrie lithique comprenant plusieurs éclats et un nucléus. Cette couche semble correspondre à un dépotoir.

Le locus 5 présente une organisation différente de celle des autres locus. Il semblerait ici qu'il puisse être rattaché à une zone de plein air comprenant des structures de stockage (vase silo, éventuel grenier de plan incomplet, matérialisé par des trous de poteaux, petite fosse dépotoir). La présence d'industrie lithique de type détritique dans le remplissage des structures semblerait indiquer qu'une activité liée à la taille du silex a été pratiquée dans le secteur.

\section{Le locus 6}

Ce locus est situé à $2 \mathrm{~m}$ environ au nord du locus 1 et peut constituer un prolongement de celui-ci (fig. 4). Le matériel est abondant, formé de céramique, d'industrie lithique et de rares fragments de faune. Contrairement aux autres locus, le locus 6 ne présente pas d'effets de parois, ni de vases écrasés en place et autres zones de dispersion inégale de mobilier. Ce fait peut être dû à une lecture au sol contrariée par une surface d'étude incomplète puisque le locus se prolonge sous la limite de fouille occidentale. Aucune structure n'est attestée dans sa partie visible.

La présentation du site laisse entrevoir un habitat dans un excellent état de conservation, chose rare dans la région pour des sites de plein air. La conservation du niveau archéologique et les effets de parois s'associant à la répartition spatiale du mobilier sont autant d'éléments permettant d'esquisser l'organisation spatiale et architecturale du site.

\section{L'ORGANISATION SPATIALE ET ARCHITECTURALE DU SITE}

L'essentiel du gisement de La Noue est formé d'un niveau archéologique comportant un mobilier chronologiquement homogène. Quelques structures en creux et une céramique enterrée viennent conférer à l'ensemble un statut de gisement en place. De fait, les hypothèses de mobilier rapporté, colluvionné, provenant d'un site voisin peuvent être écartées. Les difficultés d'interprétation du site n'en demeurent pas moins importantes et découlent pour beaucoup de l'étude du niveau archéologique. La structuration du niveau présente des zones de concentration de mobilier archéologique, des vases visiblement écrasés sur place et la distribution du mobilier montre des effets de parois souvent très nets (fig. 10 et 11). Ces effets de parois sont soumis à des constantes qui s'affirment dans l'orientation et dans les dimensions des surfaces quadrangulaires ainsi délimitées. La morphologie du niveau archéologique lui confère une épaisseur très faible, réduite à celle des tessons qui forment le niveau et qui ne se superposent que dans le cas des vases écrasés sur place. Les tessons apparaissent le plus fréquemment en position horizontale sur le sol de graviers. Ces caractères nous semblent davantage conformes à un niveau d'occupation associé à un site structuré qu'à un niveau formé de rejets en périphérie d'un site d'habitat. La distribution des éléments de faune, très inégale car ces éléments sont majoritairement situés dans les fosses dépotoir alors que le niveau archéologique ne recèle que de rares esquilles brûlées, plaide également en faveur de l'hypothèse d'un site d'habitat (fig. 12). L'étude de l'organisation spatiale du site est dès lors susceptible d'apporter des indications sur l'habitat.

\section{L'organisation spatiale du site}

L'étude du niveau archéologique laisse apparaître six locus, définis soit par une forte concentration de mobilier 


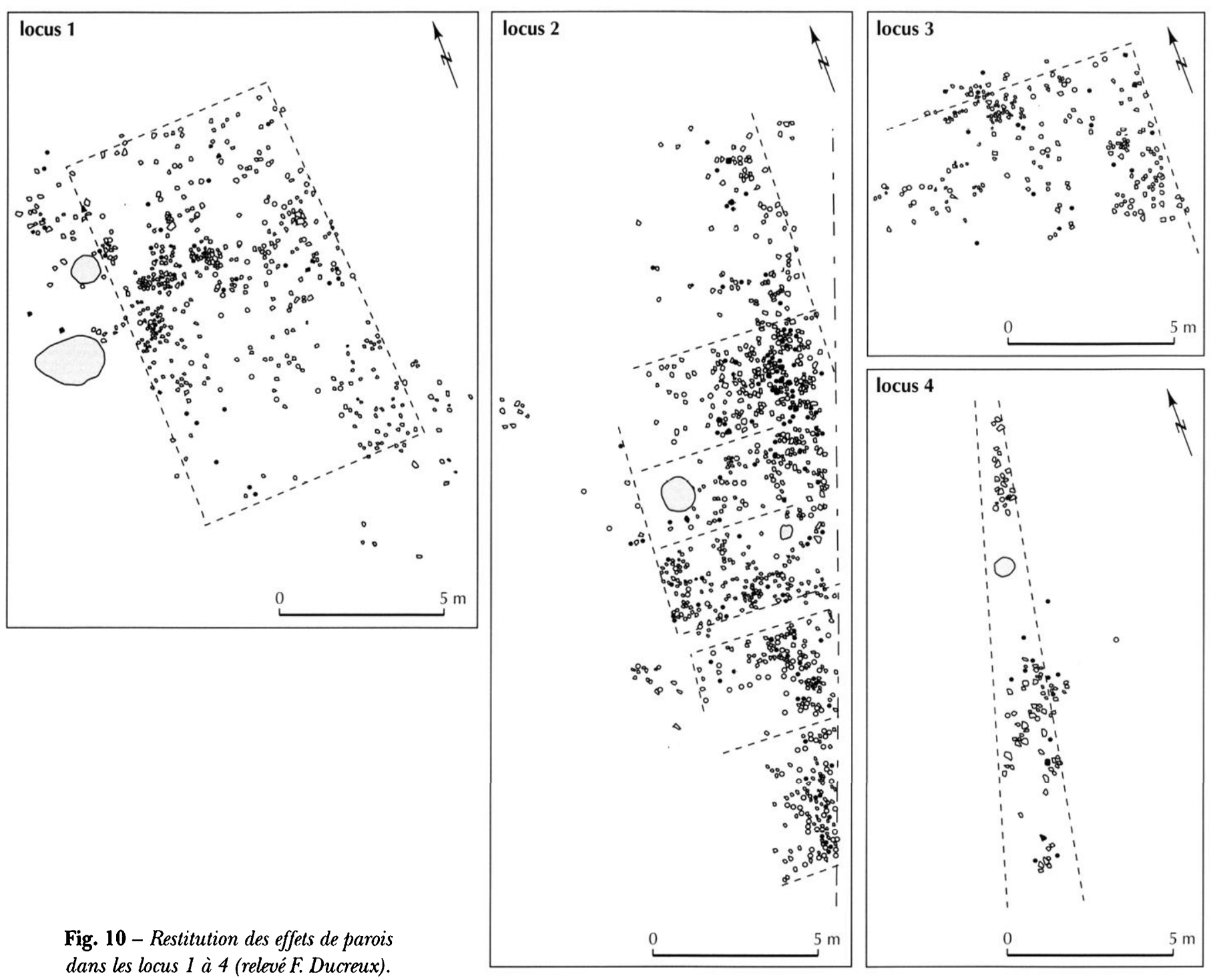

associée à des effets de parois très nets (locus 1 à 4 et 6 ), soit par une forte concentration de structures en creux, comme c'est le cas pour le locus 5 (fig. 9). Il est bien sûr tentant de voir dans les locus délimités par des effets de parois les traces de constructions. Le locus 5, qui dispose de quatre structures en creux, serait, quant à lui, une zone extérieure destinée à diverses activités, telles que le stockage (gros vase enterré encore en place, existence possible d'un grenier) ou la taille du silex (plusieurs éclats et un nucléus ont été retrouvés dans le remplissage des structures, constituant la majeure partie du mobilier). La figure 11 fait apparaître un certain nombre de constantes dans l'organisation générale des effets de parois de cinq des six locus, d'une part dans leur orientation et d'autre part dans les dimensions calculées lorsque celles-ci sont lisibles. Les surfaces isolées sont quadrangulaires; elles mesurent environ $10 \mathrm{~m}$ de longueur et $7 \mathrm{~m}$ de largeur. Elles sont orientées dans le sens de leur longueur, selon un axe nordsud, presque collées les unes aux autres sur trois rangées parallèles. Le module de quatre de ces espaces peut ĉtrc partiellement reconstitué, ceux des locus 1 et 2 étant les mieux conservés. Une telle organisation spatiale n'est pas sans rappeler celle des habitats situés en milieu humide. Pour exemple, nous citerons les sites du lac de Zurich, notamment celui de Mozartstrasse daté du Bronze ancien, dont l'organisation offre des comparaisons frappantes avec le site de La Noue (Ruoff, 1996). Ces comparaisons se situent aussi bien dans l'organisation spatiale du site (présence de grenier et zones de stockage en périphérie d'habitat) que dans la structuration de la zone habitée 


\section{Restitution des espaces}

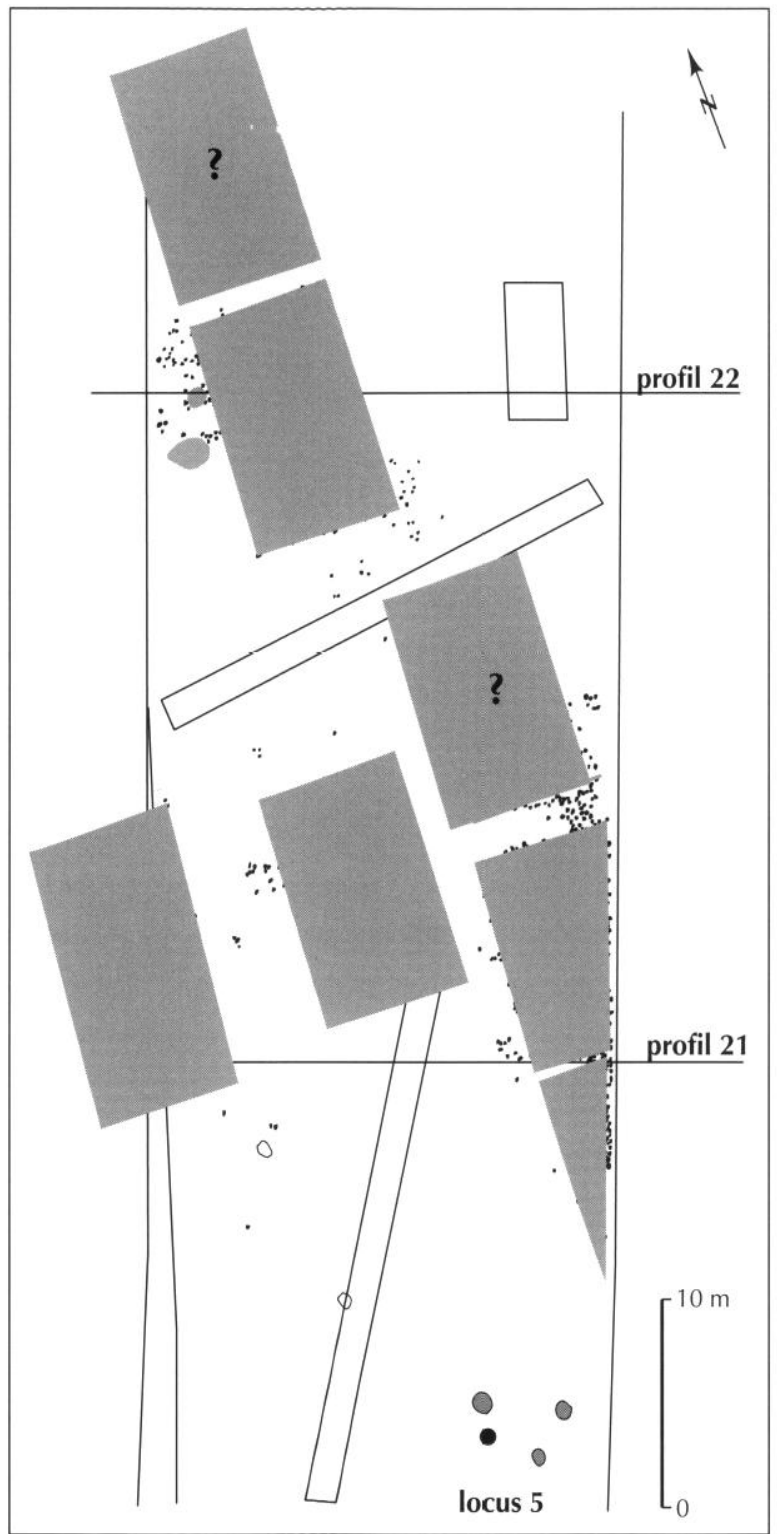

Effets de parois constatés

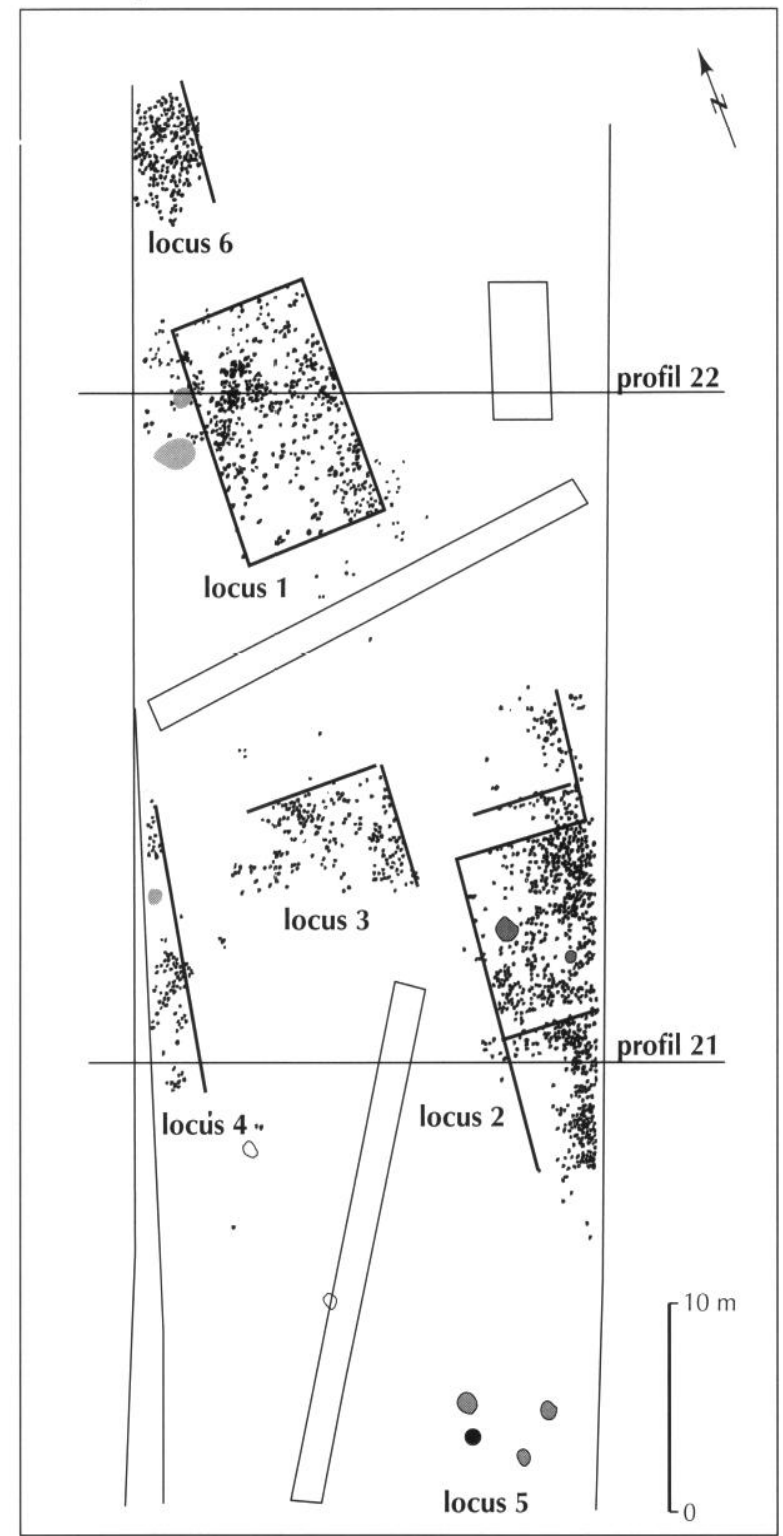

Fig. 11 - Plan interprétatif et restitution possible des espaces habités (relevé F. Ducreux).

(bandes parallèles de bâtiments collés les uns aux autres). Plus près du val de Saône, les villages implantés sur les berges du lac de Chalain (Jura), affichent également une organisation proche de celle du site de La Noue (Pétrequin dir., 1997).

La plupart des comparaisons se référant à des sites lacustres ou édifiés en milieu humide, il nous paraît nécessaire de rappeler que le site de La Noue présente les caractéristiques d'un site terrestre et des structures propres à ce type de gisement (fosses dépotoirs creusées dans le substrat géologique, vases silos enterrés). Néanmoins, l'adoption d'une architecture de milieu humide semble ici dictée par la proximité des berges du lac des Orlans ou d'un hypothétique bras de la Saône.

De façon générale, le site de La Noue tranche très nettement, de par son organisation spatiale, avec les modèles d'habitats terrestres campaniformes connus. Ceuxci, qu'ils soient régionaux ou extra-régionaux, témoignent dans la plupart des cas d'un habitat dispersé, ne regroupant guère plus de deux unités, pas toujours contemporaines. 


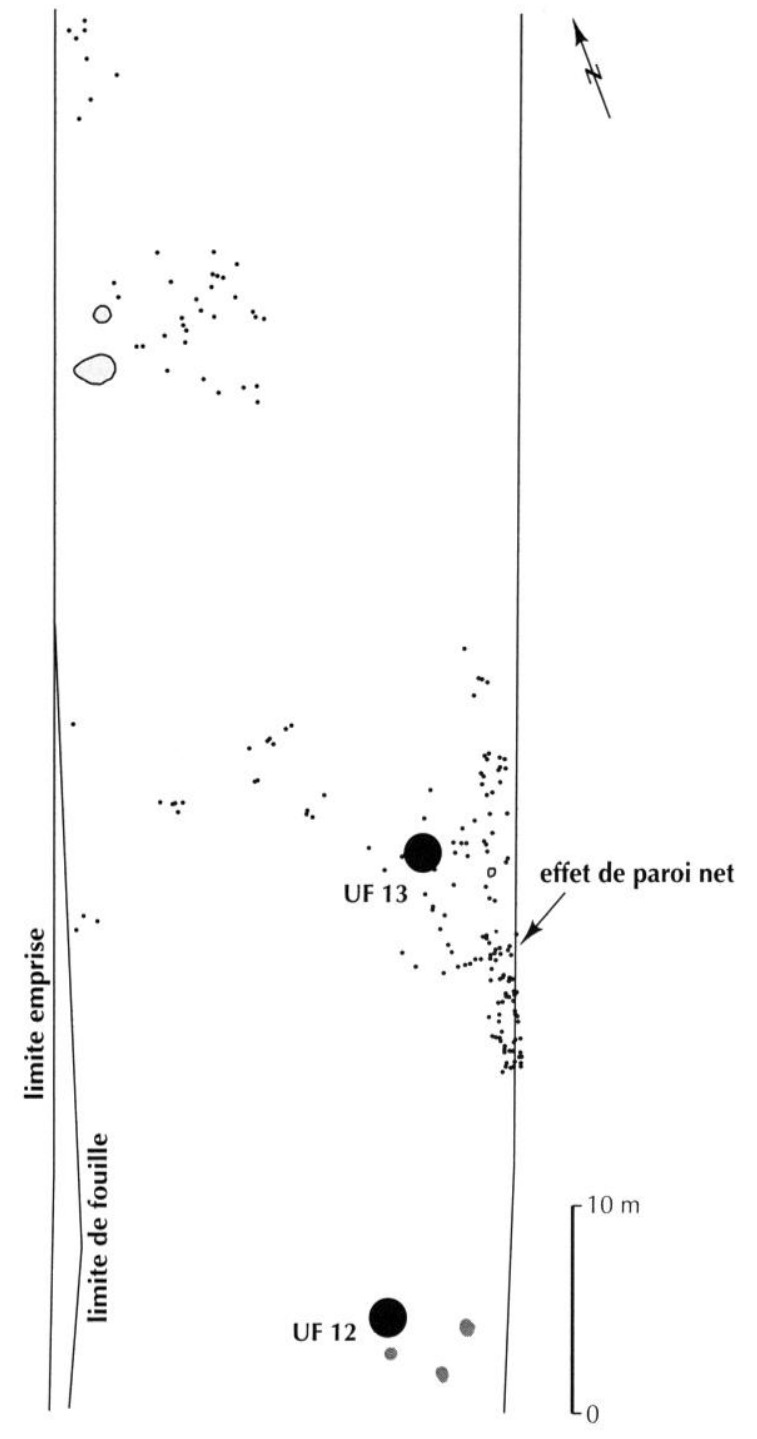

... mobilier sous forme d'esquilles osseuses carbonisées

plus de 10 fragments bien conservés et non brûlés (contexte de dépotoir)

Fig. 12 - Répartition de la faune dans les structures et dans le niveau archéologique.

C'est le cas pour les sites des Calades (Orgon, Bouches-duRhône), de Bois Sacré (Saint-Côme-et-Maruejols, Gard) (Barge-Mahieu, 1989; Roudil, 1992). Seul l'habitat de Champ-Vully (Rances, canton de Vaud, Suisse) est, avec le plan de 5 habitations, certaines accolées à d'autres, susceptible de présenter des comparaisons intéressantes autant dans l'organisation générale de l'habitat que dans son modèle architectural (Gallay et al., 1980).

\section{Étude architecturale}

Les 5 unités d'habitation de La Noue présentent des caractéristiques homogènes et répondent donc aux mêmes principes architecturaux. La caractéristique architecturale majeure de l'ensemble est l'absence de traces de poteaux et la présence d'effets de parois délimitant des espaces habités. La bonne lisibilité des structures en creux existantes nous conduit à rejeter l'hypothèse d'une absence de trous de poteaux consécutive à une mauvaise lecture au sol. Plusieurs techniques architecturales, mettant en œuvre des solutions alternatives à des poteaux fichés en terre peuvent dès lors être évoquées.

Une architecture légère, de type tentes, peut avoir été employée. La mise en œuvre d'une telle solution est simple (toile, branchages) mais ne permet pas d'établir une zone d'habitat durable. Il n'est pas certain, d'autre part, que les effets de parois aient pu être conservés dans ce cas. Un tel campement, par nature limité dans le temps, ne peut être qu'attribué à une population à caractère nomade ou seminomade. Or, comme nous le verrons dans l'étude du mobilier, l'abondance de la céramique (plus de 371 individus pour 5 unités d'habitation fouillées partiellement), plaide en faveur d'un habitat stable ayant eu une durée de vie assez longue.

La seconde solution retenant notre attention est une technique utilisée de la fin du Néolithique jusqu'au Bronze moyen, sur l'ensemble de l'arc alpin, aussi bien dans le domaine lacustre que dans le domaine terrestre. Cette technique consiste à édifier le bâtiment sur un cadre constitué de poutres de bois (troncs d'arbres ou poutres sablières) posées à même le sol. Les poteaux formant l'ossature du bâtiment sont alors fichés dans le cadre en place par l'intermédiaire de mortaises. Dans le locus 2, l'étude de la répartition des esquilles osseuses permet de mettre en évidence un effet de paroi interne au locus et perpendiculaire à sa longueur (fig. 12). Cette observation est importante car elle permet de mettre en évidence un découpage de l'espace interne au locus. Un plancher, également posé sur ce cadre, présente une isolation efficace du bâtiment contre les effets indésirables d'un sol gorgé d'eau, mais également contre les intrusions animales.

Cette méthode était donc très employée sur les habitats en milieu humide en raison de la protection qu'elle procure en cas d'inondation de faible amplitude. Elle permet l'édification de bâtiments à division interne, accolés les uns aux autres, disposant d'un toit à deux pans. Un exemple assez 
complet de ce type d'habitat est donné par le site lacustre de Zurich Mozartstrasse qui propose en outre une organisation spatiale proche de celle de La Noue (fig. 10). La même technique est également attestée sur le littoral du lac de Chalain (Pétrequin dir., 1997). Hors du domaine lacustre, l'habitat de Champ-Vully à Rances (canton de Vaud, Suisse), est formé de 5 maisons installées sur une terrasse morainique, sur des cadres de bois posés à même le sol et bloqués par des galets. Cette comparaison est d'autant plus intéressante que sa datation est contemporaine (chronologiquement et culturellement) de celle de La Noue.

\section{Campement de courte durée ou habitat durable sur ossature de bois?}

Pour apporter un début de réponse à la question de la durée de l'occupation, il est nécessaire de croiser les deux hypothèses émises plus haut avec l'inventaire global du mobilier. Comme nous l'avons déjà souligné, la quantité importante de céramique retrouvée en association directe avec les locus 1 à 5 , considérés comme des unités d'habitation, ne plaide pas en faveur d'un habitat de courte durée, sauf si celui-ci est voué à la fabrication et à la diffusion de céramique. Dans ce cas, des déchets de fabrication (céramiques déformées ou sur-cuites, parois de four) auraient inévitablement été découverts. Or, les seules céramiques portant des traces de sur-cuisson ont été brûlées après utilisation en foyer domestique. Les mêmes traces de feu se remarquent sur quelques éléments d'industrie lithique. La quantité d'objets lithiques et de restes fauniques contraste cependant avec le mobilier céramique (voir p. 51-52). En effet, le nombre d'outils retrouvés reste très faible et le nombre d'animaux identifiés l'est encore plus (voir p. 50). Cet aspect de l'ensemble mobilier s'accorde davantage à un site affecté à une occupation de courte durée, mais peut également trouver une explication dans la distribution spatiale du mobilier sur l'ensemble du gisement, qui, rappelons-le, n'a pas été fouillé sur toute sa superficie. Il est toutefois intéressant de noter que cette constatation s'accorde avec d'autres sites d'habitats chalcolithiques.

L'exemple du site fontbuxien de Boussargues (Argelliers, Hérault), dont le niveau d'occupation a été particulièrement bien conservé, montre une disproportion très nette entre le mobilier céramique et le reste du mobilier (faune et lithique) (Colomer et al., 1990). De même qu'à La Noue, l'industrie lithique est représentée à Boussargues par une très grande majorité d'éclats de taille. Le site d'habitat campaniforme de Muret (Haute-Garonne), malgré un nombre important d'éclats découverts dans le niveau d'habitat conservé, n'a livré qu'une armature de flèche, une vingtaine de grattoirs, 8 fragments de lames pour l'outillage le mieux représenté (Jolibert, 1988). La faune n'y est représentée que par 12 fragments concernant quatre espèces animales.

S'il est donc, dans l'état actuel des recherches, difficile de trancher nettement en faveur de l'une ou de l'autre hypothèse, notre préférence se tourne malgré tout vers un habitat à caractère durable, étudié pour résister aux crues, car une majorité d'arguments s'accorde avec ce type de site: - il est difficile d'établir un type de site de campement en zone humide et éventuellement inondée;

- le nombre important de céramiques est en désaccord avec un campement;

- la conservation des effets de parois est en désaccord avec un campement;

- le remplissage des structures en creux montre des phases de dépôt s'intercalant avec des phases de comblement naturel de puissance plus importante; ceci évoque un rythme d'occupation assez long, où les périodes d'occupation s'intercalent avec des phases naturelles pouvant évoquer des inondations.

Si l'hypothèse d'un habitat à caractère durable semble la plus probable, il est intéressant de discuter des modalités de son occupation. En effet, la présence d'habitations stables, construites en matériaux lourds, n'implique pas automatiquement une occupation continue. Une autre forme d'occupation, saisonnière, peut également être envisagée. L'agriculture peut être pratiquée sur le site en période estivale tandis que la période hivernale voit un repli de la population à l'abri des crues de la Saône. Ce rythme saisonnier a pu se maintenir jusqu'à ce que le site de La Noue soit rendu impraticable et de ce fait abandonné.

\section{Réflexions sur l'abandon et sur la conservation du site}

Si l'habitat de La Noue a été conçu pour durer, des éléments tels que l'abandon en place des céramiques de stockage, l'importance du mobilier céramique lié aux habitats, indiquant que le site n'a pas été nettoyé, suggèrent un abandon rapide et précipité du site. Une inondation plus importante que la moyenne, ayant submergé les habitations au-delà du niveau de leur plancher et ayant provoqué des dommages dont l'importance justifie l'abandon du site, est possible; ce type de phénomène survient encore régulière- 
Tabl. I - Détermination des restes osseux du site de La Noue.

Sus domesticus : porc domestique; Bos taurus : bœuf; Lepus europaeus : lièrere commun.

\begin{tabular}{|c|c|c|c|c|c|c|c|c|c|}
\hline Zones & $\begin{array}{l}\text { Référence } \\
\text { du site }\end{array}$ & $\begin{array}{l}\text { Nombre } \\
\text { de restes }\end{array}$ & $\begin{array}{c}\text { Masse totale } \\
\text { correspondante } \\
\text { (en g) }\end{array}$ & $\begin{array}{c}\text { Dont : nombre de } \\
\text { restes avec trace } \\
\text { de combustion }\end{array}$ & $\begin{array}{c}\text { Dont : nombre } \\
\text { de restes avec } \\
\text { traces } 3 \mathrm{D}\end{array}$ & $\begin{array}{c}\text { Dont : nombre } \\
\text { de restes } \\
\text { déterminables }\end{array}$ & $\begin{array}{l}\text { Leur masse } \\
\text { (en g) }\end{array}$ & Espèces & NMIE * \\
\hline Zone 2 & UF 13 & 29 & 122 & 5 & 0 & 8 & 74 & $\begin{array}{l}\text { Sus domesticus } \\
\text { Bos taurus }\end{array}$ & $\begin{array}{l}1 \\
1\end{array}$ \\
\hline Zone 2 & $\begin{array}{l}\text { Zone } 2 \\
\text { niveau } \\
\text { faune brûlée }\end{array}$ & 219 & 112 & tous très brûlés & 0 & 2 & 2 & Sus domesticus & 1 \\
\hline Zone 2 & $\begin{array}{l}\text { UF } 16 \\
\text { Passe } 3\end{array}$ & 1 & - & - & - & - & - & $\begin{array}{l}\text { fragment de tige } \\
\text { de Crinoïde, } \\
\text { calcaire secondaire }\end{array}$ & - \\
\hline Zone 2 & $\begin{array}{l}\text { UF } 29 \\
\text { Passe } 1\end{array}$ & 13 & 42 & 0 & 0 & 1 & 4 & Bos taurus & 1 \\
\hline Total zone 2 & & 262 & 276 & 224 & 0 & 11 & 80 & & 4 \\
\hline Zone 5 & UF 12 & 372 & 298 & 199 & 0 & 14 & 52 & $\begin{array}{l}\text { Sus domesticus } \\
\text { Bos taurus } \\
\text { Lepus europaeus } \\
\text { (charbons de bois) }\end{array}$ & $\begin{array}{l}2 \\
2 \\
1\end{array}$ \\
\hline Total & & 634 & 574 & 423 & 0 & 25 & 132 & & 9 \\
\hline
\end{tabular}

* Nombre minimum d'individus exprimé

ment de nos jours dans le secteur. La durée d'occupation réelle de cet habitat reste difficile à évaluer, mais peut être de l'ordre d'une ou plusieurs années.

Il est, à ce stade de l'étude du gisement, légitime de s'interroger sur les conditions ayant permis la conservation d'un site d'habitat avec son niveau archéologique dans un milieu soumis à des inondations continuelles. Celles-ci peuvent entrainer le délavement complet d'un site et la dispersion du mobilier sur une grande surface.

La situation du gisement dans une dépression est un premier élément de réponse. Les effets de l'érosion naturelle y sont certainement moins sensibles que si le site s'était trouvé en bordure de terrasse ou en limite de zone inondable. Les dépôts de limons dus aux inondations ont, dans ce cas précis, très bien pu sceller le gisement et ainsi éviter l'altération du niveau archéologique. Cet argument ne permet pas à lui seul d'expliquer l'excellente conservation du niveau archéologique. Les effets de parois, reconnus sur toutes les unités d'habitation, se sont formés alors que la base des bâtiments était encore en place. Les planchers des habitations ont dû se dégrader rapidement après l'abandon du site, permettant ainsi le blocage du mobilier à l'intérieur du cadre constituant la base du bâti. Les dépôts de limon par les inondations ont certainement permis la conservation du mobilier dans cet état.

F. D.

\section{LES RESTES DE FAUNE}

\section{INVENTAIRE ET DÉTERMINATION}

Les restes de faune relevés sur le site de La Noue ne constituent pas une série importante: 634 restes pour un poids total de $574 \mathrm{~g}$ (tabl. I). La faune n'a été découverte que dans deux zones: la zone 2 et la zone 5 . Les déterminations, réalisées avant l'étude du site pour permettre l'envoi rapide d'échantillons à dater, ont permis de dresser un premier inventaire, présenté ici par espèce ${ }^{4}$.

\section{- Bos taurus}

1 phalange 2 postérieure de petite taille, épiphysée, un peu endommagée,

1 fragment brûlé d'émail de molaire indéterminable,

des fragments de molaires indéterminables (sauf un de M3/g. sûre),

$1 \mathrm{M} / 3 \mathrm{~g}$., individu assez âgé,

$1 \mathrm{M} / \mathrm{lg}$., fragment de paroi interne.

- Sus domesticus

1 omoplate d. partielle,

1 fragment d'omoplate g., jeune individu,

4. 31) signific traces de dépeçage, découpe, dé carnisation; d. = droit, g. = gauche.

Pour une dent, par exemple: $I 2 /=2^{c}$ incisive supérieure, $I / 2=2^{\prime}$ incisive inférieure. 
1 diaphyse d'humérus $\mathrm{d}$. cassée (épiphysation impossible à voir),

1 phalange 3 de doigt latéral indéterminable; très fortement brûlée,

1 fragment d'extrémité distale de métapode indéterminable, très fortement brûlé,

1 fragment d'extrémité distale de phalange 2 très brûlée, $1 \mathrm{I} 2 / \mathrm{g}$. de bonne taille, avec usure sensible,

1 fragment de maxillaire sup. g. avec $\mathrm{Ml} /, \mathrm{M} 2 /$; grande taille et un peu d'usure,

1 fragment de M3/g., partie postérieure,

$1 \mathrm{M} / 2$ complète, $\mathrm{d}$., non usée,

1 fragment d'émail de $12 / \mathrm{d}$. (dent définitive),

$1 \mathrm{M} / 3$, cassée, non usée,

$1 \mathrm{PM} / 3 \mathrm{~d}$., entière, un peu usée,

$1 \mathrm{PM} 2 / \mathrm{g}$. entière.

- Lepus europaeus

1 fragment de diaphyse de tibia d., jeune individu.

- Herbivore indéterminable: fragments de molaire.

\section{QUELQUES COMMENTAIRES}

Le matériel souvent très brûlé rend pratiquement impossible l'observation d'éventuelles traces de surface. La recherche systématique d'informations de tracéologie n'apporte donc rien dans ce cas et les résultats négatifs sur le tableau I ne sont pas significatifs.

La faune déterminable se résume aux deux espèces domestiques souvent rencontrées, le porc et le bœuf. Aucune confusion n'est possible ici avec Sus scrofa, le sanglier, ni avec Bos primigenius, l'aurochs. Une indication unique de lièvre commun (Lepus europaeus) dans l'UF 12 apporte une preuve de la pratique de la chasse, au moins au petit gibier. Bien que l'on ne dispose que de peu d'éléments, la part de l'élevage devait être quand même largement majoritaire à La Noue. Il est difficile de proposer un nombre minimum d'individus consommés, comme on le. fait d'habitude en archéozoologie. Les restes de porc domestique (Sus domesticus) peuvent tout à fait provenir du même animal et non pas de quatre animaux différents. De même, les restes de bœuf (Bos taurus) n'apportent pas de preuve objective de la présence de plusieurs individus. Comme pour le porc, tous les éléments de bœuf peuvent correspondre au même animal car toutes les informations quant à la taille, à la latérisation des pièces, au degré d'usure des dents restent compatibles avec la présence d'un unique individu.
Il semble difficile d'aller au-delà de ces simples commentaires avec si peu de matériel déterminable et d'impossibles observations taphonomiques dues à une combustion intense.

A. A.

\section{DATATIONS ABSOLUES}

Au total, 6 échantillons d'os de faune ont été traités. Après un nettoyage de surface par brossage et grattage, et un passage aux ultrasons dans des solutions d'eau distillée, les échantillons sont séchés puis broyés. Le protocole d'extraction de la matière organique, le collagène, mis en œuvre est inspiré de celui de Longin (1971). Ce protocole assure une élimination efficace du carbone contaminant qu'il soit d'origine minérale ou organique.

Les 4 premiers échantillons ont été traités en appliquant ce protocole rigoureusement et n'ont pas donné de collagène. Pour les 2 derniers échantillons, le protocole a été un peu modifié: l'étape de décontamination par passage dans un bain de soude n'a pas été appliquée. Le poids de collagène obtenu a été, dans les deux cas, très faible. La datation des échantillons par SMA (spectrométrie de masse couplée à un accélérateur) a quand même été tentée: les échantillons ont été envoyés au laboratoire d'Oxford (Angleterre).

Les datations obtenues sont:

- Lyon-2736(OxA) : $1555 \pm 40 \mathrm{BP}$, soit de 419 à 601 apr. J.-C.

- Lyon-2737(OxA) : $1965 \pm 35 \mathrm{BP}$, soit de 42 av. J.C. à 125 apr. J.-C.

Ces deux dates sont beaucoup trop jeunes pour correspondre à du Campaniforme. Le rajeunissement constaté peut être expliqué en grande partie par le fait que le collagène a été très mal conservé et que la décontamination n'a pas pu être faite en totalité.

C. $\mathrm{O}$.

\section{ANALYSE DU MOBILIER}

\section{LA CÉRAMIQUE}

\section{COMPOSITION ET RÉPARTITION DU CORPUS}

La série céramique découverte sur le site de La Noue comprend 2592 tessons qui représentent un peu plus de $28 \mathrm{~kg}$ (tabl. II). Elle a en outre livré des fragments de boule d'argile (fig. 13, n 2).

Les tessons ont été répartis en deux catégories: l'une correspond aux individus vases que nous avons pu isoler, 
Tabl. II - Composition du corpus céramique de La Noue.

\begin{tabular}{|c|c|c|c|c|c|c|c|c|}
\hline \multirow[b]{2}{*}{ Zone } & \multirow[b]{2}{*}{ UF } & \multicolumn{2}{|c|}{ Céramique commune } & \multicolumn{2}{|c|}{ Céramique décorée } & \multicolumn{2}{|c|}{ Lot } & \multirow{2}{*}{$\%$ poids tota } \\
\hline & & nombre tessons & poids en g. & nombre tessons & poids en g. & nombre tessons & poids en $\mathrm{g}$. & \\
\hline HS* & & 21 & 605 & 8 & 61 & 182 & 2058 & 10 \\
\hline $\mathrm{Z1}$ & 1 & 8 & 99 & 6 & 40 & 31 & 369 & \\
\hline $\mathrm{Z1}$ & 2 & 3 & 52 & 27 & 536 & 57 & 526 & \\
\hline $\mathrm{Z1}$ & 3 & 10 & 190 & 3 & 61 & 59 & 359 & \\
\hline $\mathrm{Z1}$ & 15 & 6 & 101 & 3 & 42 & 18 & 142 & \\
\hline $\mathrm{Z1}$ & 17 & 3 & 59 & 2 & 10 & & & \\
\hline $\mathrm{Z1}$ & 18 & 6 & 94 & 16 & 174 & 65 & 615 & \\
\hline Z1 & 19 & 32 & 755 & 13 & 96 & 69 & 676 & \\
\hline $\mathrm{Z1}$ & 22 & 18 & 208 & 7 & 53,5 & 80 & 839 & \\
\hline $\mathrm{Z1}$ & 26 & 8 & 93 & 2 & 11 & 24 & 344 & \\
\hline Total zone 1 & & 94 & 1651 & 79 & 1023,5 & 403 & 3870 & 23 \\
\hline $\mathrm{Z2}$ & 13 & 13 & 221 & 1 & 4 & 153 & 1351 & \\
\hline $\mathrm{Z} 2$ & 16 & 23 & 347 & 21 & 181,5 & 138 & 1278 & \\
\hline $\mathrm{Z} 2$ & 20 & 4 & 34 & 1 & 5 & 92 & 580 & \\
\hline $\mathrm{Z2}$ & 21 & 6 & 109 & 2 & 13 & 62 & 487 & \\
\hline $\mathrm{Z2}$ & 23 & 3 & 52 & 2 & 34,5 & 73 & 521 & \\
\hline $\mathrm{Z2}$ & 24 & 12 & 237 & 15 & 136 & 143 & 1195 & \\
\hline $\mathrm{Z2}$ & 27 & 20 & 484 & 20 & 180 & 149 & 1426 & \\
\hline $\mathrm{Z2}$ & 29 & 15 & 179 & 4 & 26 & 79 & 658 & \\
\hline $\mathrm{Z2}$ & 30 & 2 & 6 & 1 & 4 & 30 & 272 & \\
\hline $\mathrm{Z2}$ & 31 & 3 & 10 & & & 28 & 285 & \\
\hline $\mathrm{Z2}$ & 35 & 1 & 3 & & & & & \\
\hline Total zone 2 & & 102 & 1682 & 67 & 584 & 947 & 8053 & 35 \\
\hline $\mathrm{Z3}$ & 37 & 7 & 55 & 6 & 31,5 & 88 & 630 & \\
\hline $\mathrm{Z3}$ & 39 & 8 & 230 & 3 & 17 & 45 & 412 & \\
\hline Total zone 3 & & 15 & 285 & 9 & 48,5 & 133 & 1042 & 5 \\
\hline $\mathrm{Z} 4$ & 32 & 2 & 83,5 & & & 8 & 154 & \\
\hline $\mathrm{Z4}$ & 33 & 6 & 153,5 & & & 20 & 182 & \\
\hline $\mathrm{Z} 4$ & 34 & 12 & 266 & 2 & 17 & 71 & 918 & \\
\hline $\mathrm{Z4}$ & 40 & & & & & 4 & 19 & \\
\hline Total zone 4 & & 20 & 503 & 2 & 17 & 103 & 1273 & 6 \\
\hline $\mathrm{Z5}$ & 9 & 62 & 2994 & & & & & \\
\hline$\overline{Z 5}$ & 10 & & & & & 8 & 97 & \\
\hline $\mathrm{Z5}$ & 11 & & & & & 4 & 39 & \\
\hline $\mathrm{Z5}$ & 12 & 1 & 9 & & & 15 & 208 & \\
\hline Total zone 5 & & 63 & 3003 & 0 & 0 & 27 & 344 & 12 \\
\hline $\mathrm{Z6}$ & 41 & 23 & 301 & 24 & 171 & 270 & 1970 & \\
\hline Total zone 6 & & 23 & 301 & 24 & 171 & 270 & 1970 & 9 \\
\hline Total site & & 338 & 8030 & 189 & 1905 & 2065 & 18610 & $100 \%$ \\
\hline
\end{tabular}

* Hors stratigraphie, niveau de décapage, ramassage de surface...

l'autre est composée de tessons de petite taille, bien souvent érodés, et qui n'ont aucune caractéristique typologique particulière. Pour cette dernière catégorie, qui a été enregistrée en lots par structure, nous nous sommes contenté de peser et de compter les tessons.

Nous proposons un nombre d'individus de 371 , probablement surévalué étant donné la fragmentation des tessons. Les 371 individus représentent 527 tessons (et $9935 \mathrm{~g})$. Les lots comprennent quant à eux 2065 tessons $(18610 \mathrm{~g})$.
Au total, $80 \%$ des tessons ont été enregistrés dans les lots, $13 \%$ sont caractéristiques de la céramique commune campaniforme et $7 \%$ sont décorés (fig. 13 à 30$)^{5}$.

Signalons enfin que plusieurs tessons protohistoriques et historiques ont été découverts en surface ou dans des structures interprétées par les fouilleurs comme des sillons de labour: hors stratigraphie (37 tessons),

5. Tous les éléments typologiques ont été dessinés. Les références des tessons correspondent au numéro de l'unité de fouille suivi du numéro d'inventaire de la pièce. 

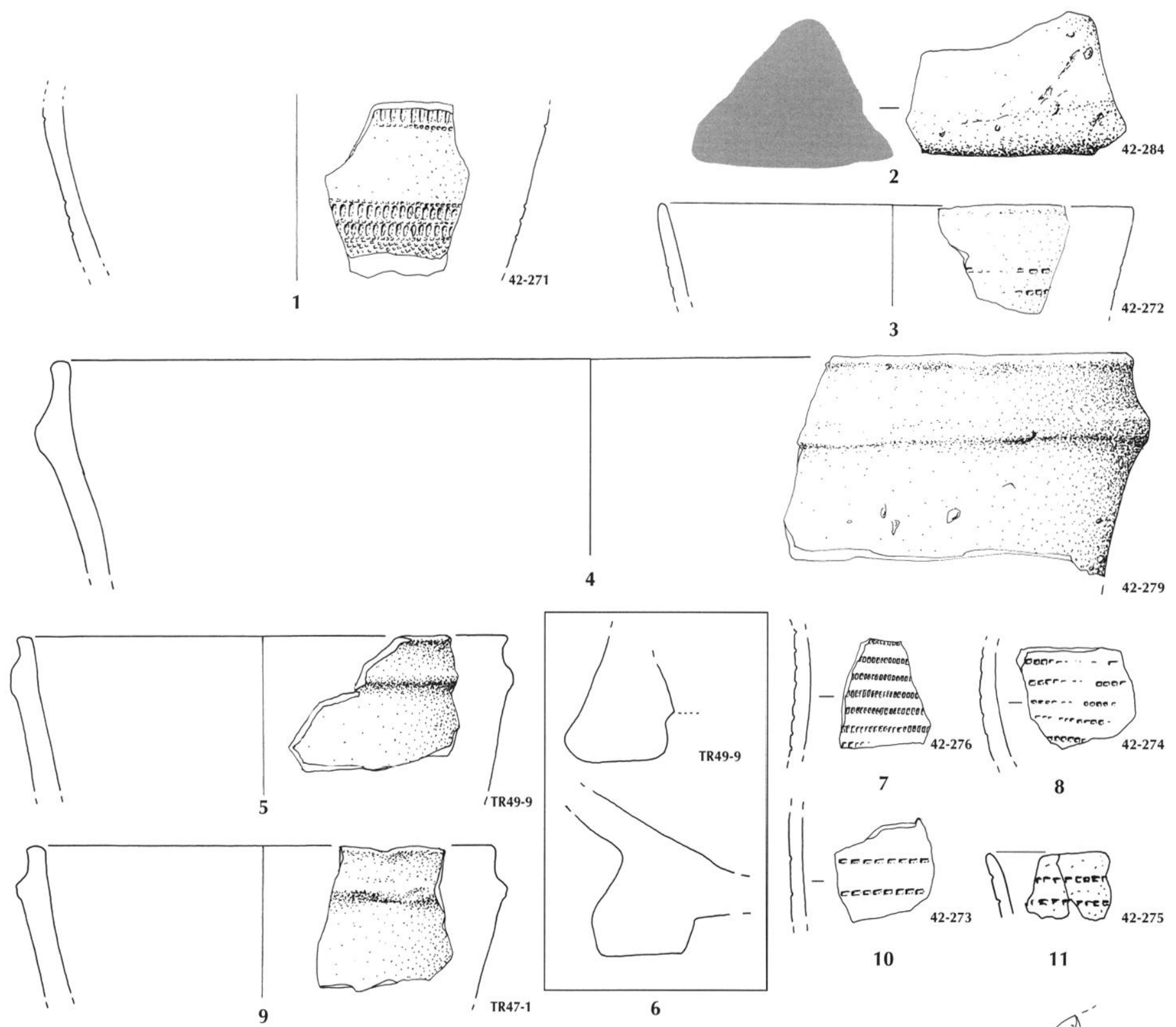

11
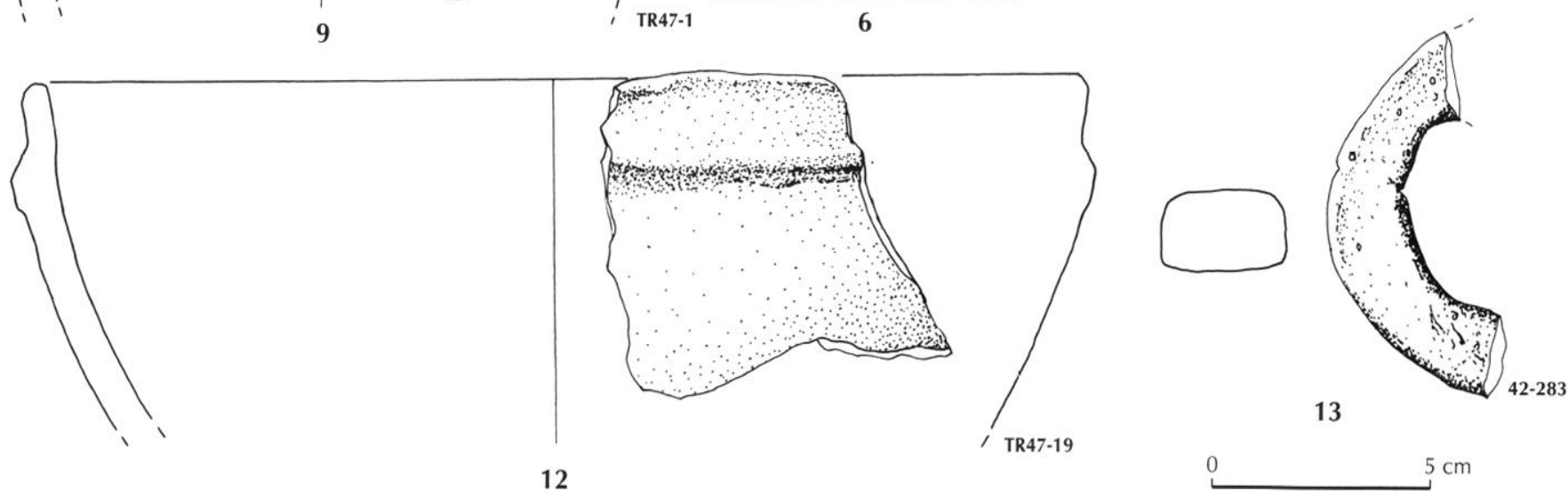

Fig. 13 - 1-13 : céramique provenant du diagnostic (TR47 et TR49) et du décapage (UF 42). Dessin F. Ducreux et F. Gauchet. 


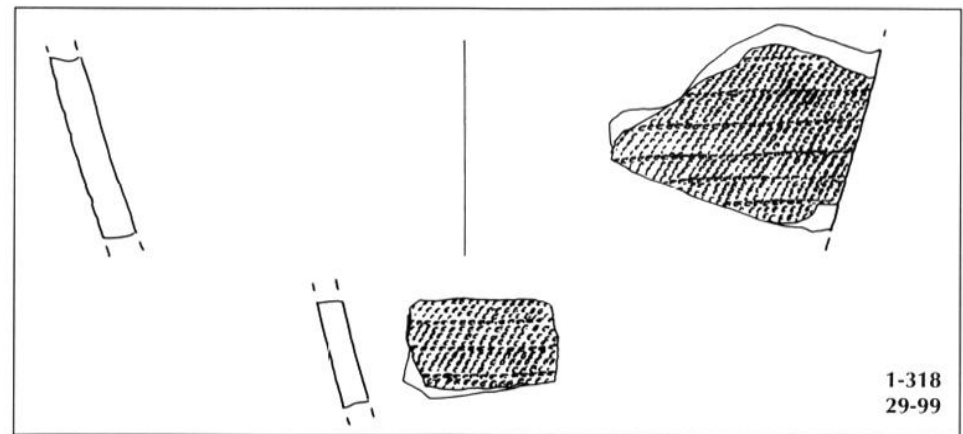

1
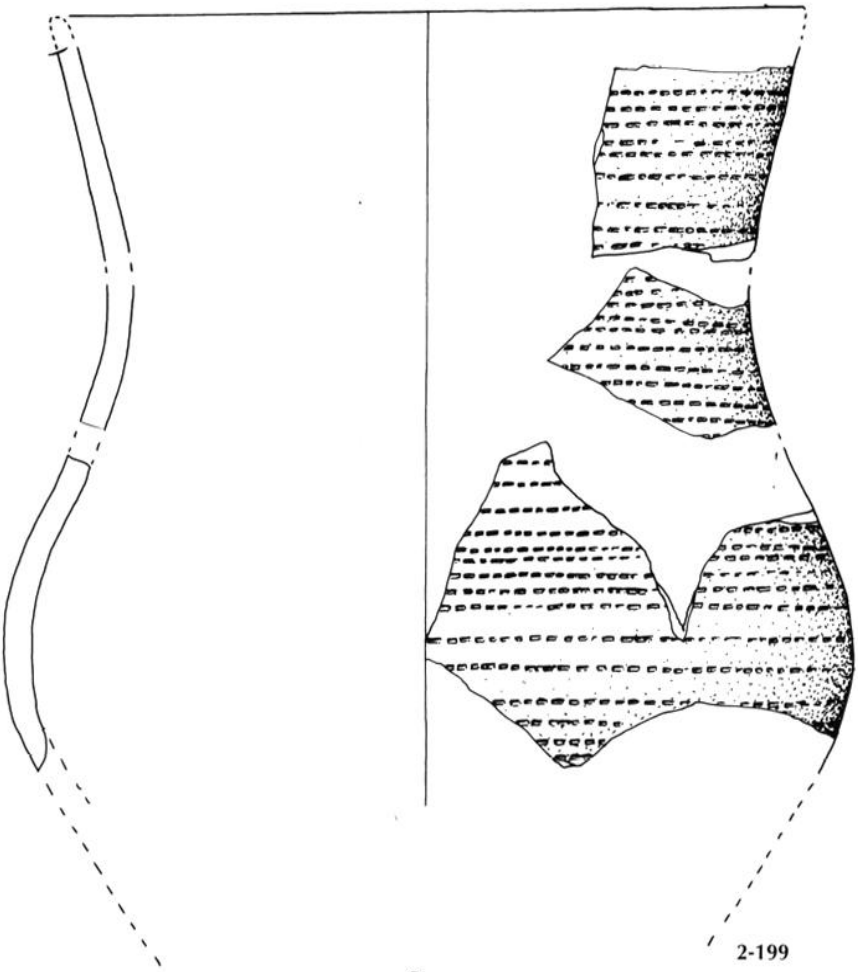

3

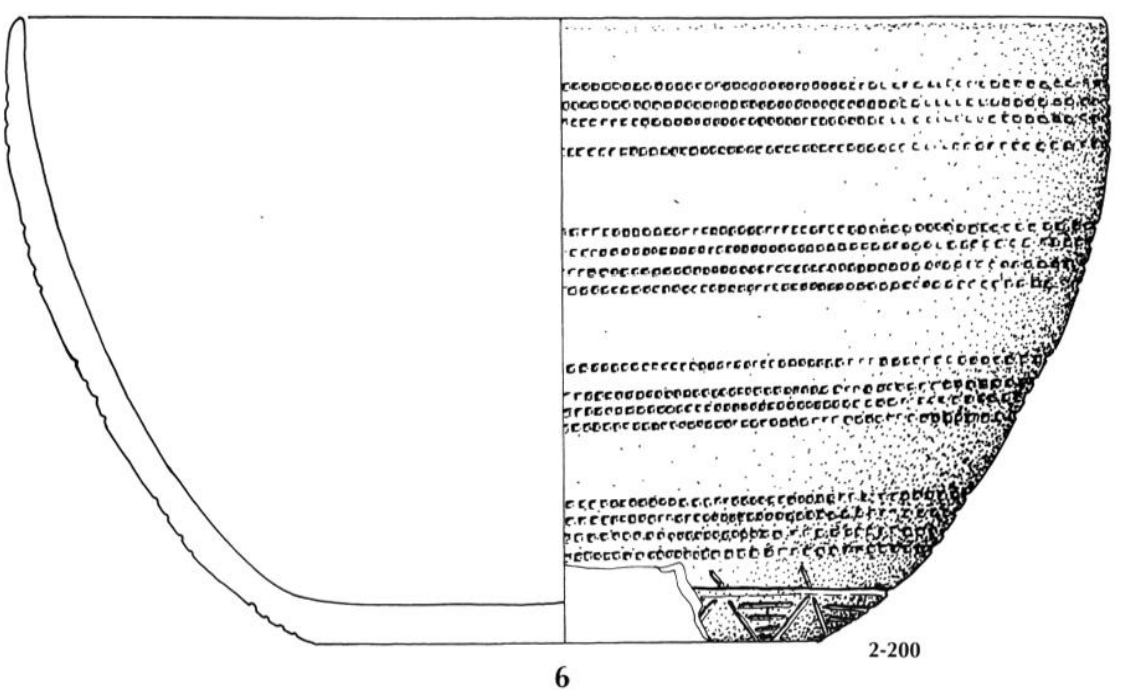

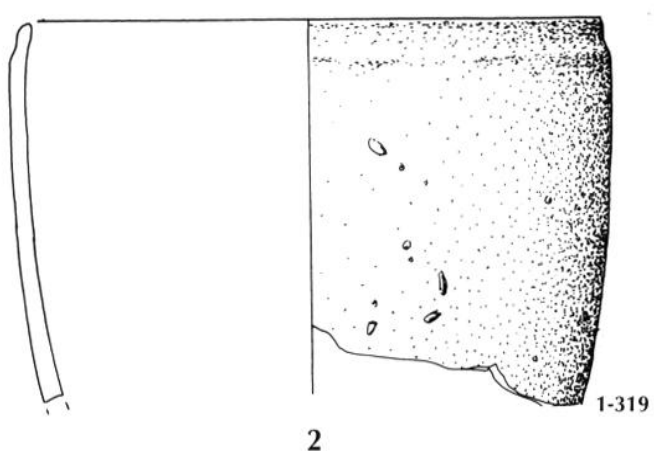

2

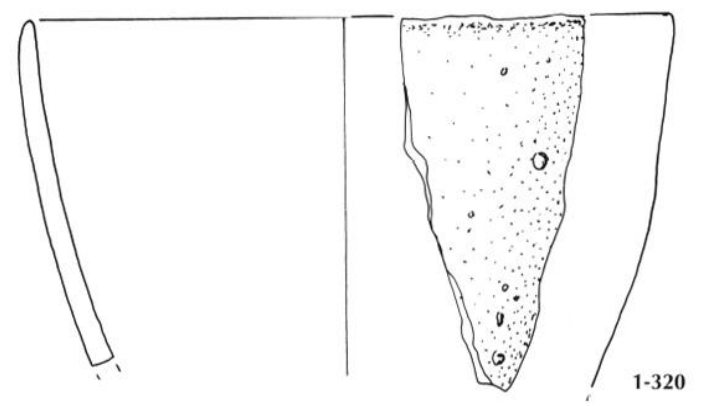

4
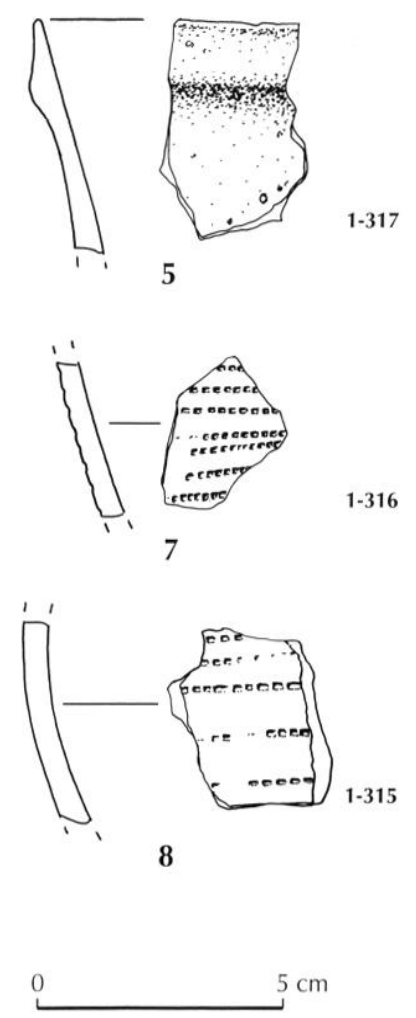

Fig. $14-1-8$ : céramique du locus 1, UF 1 et UF 2 (dessin F. Ducreux et F. Gauchet). 

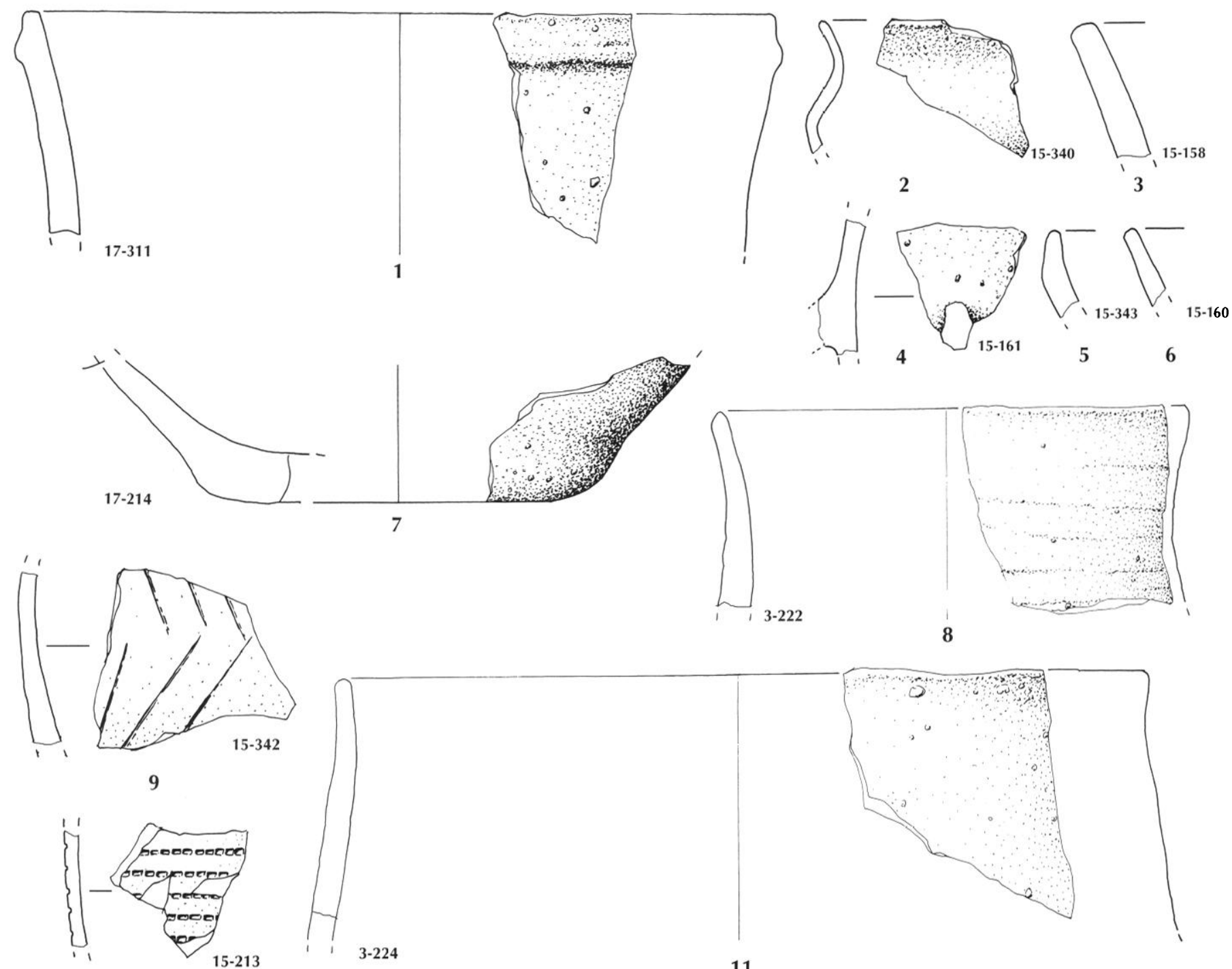

10
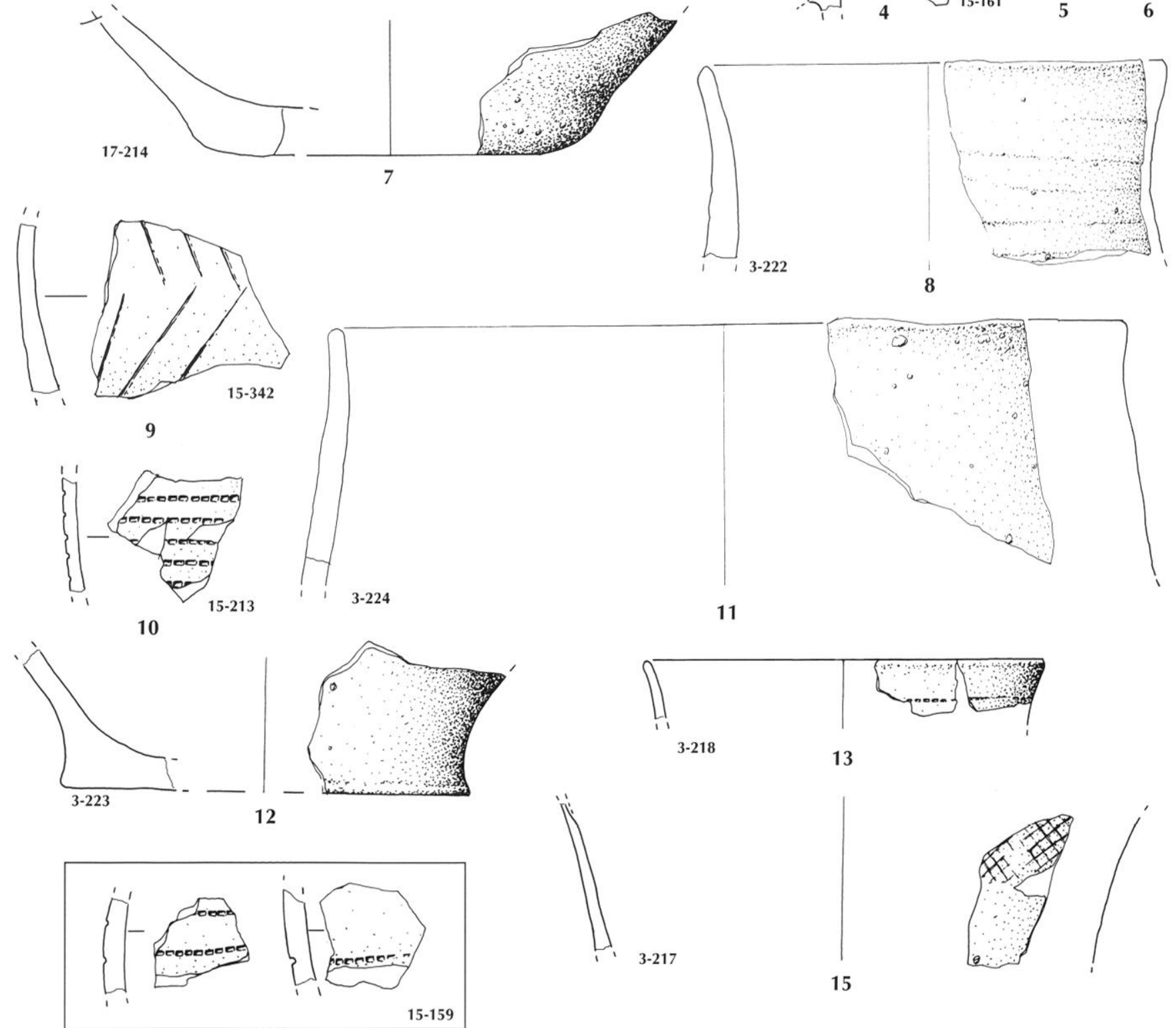

15-159

14

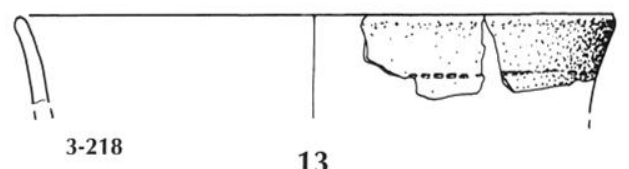

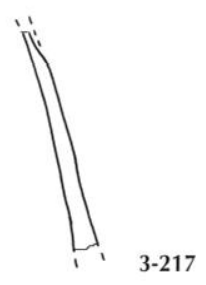

0

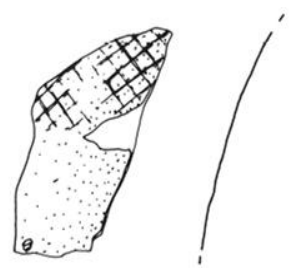

15
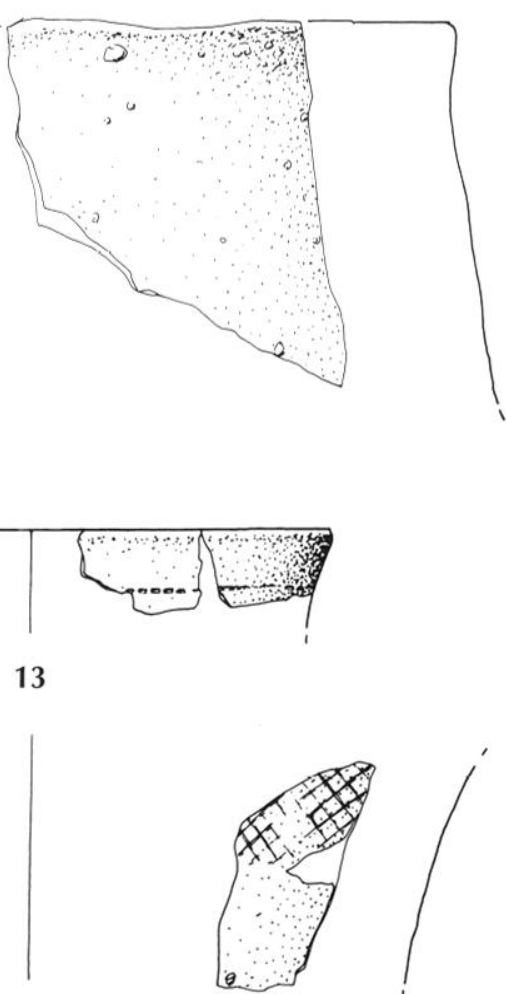
$5 \mathrm{~cm}$

Fig. 15 - 1-15: céramique du locus 1, UF 15, UF 17 et UF 3 (dessin F. Ducreux et F. Gauchet). 


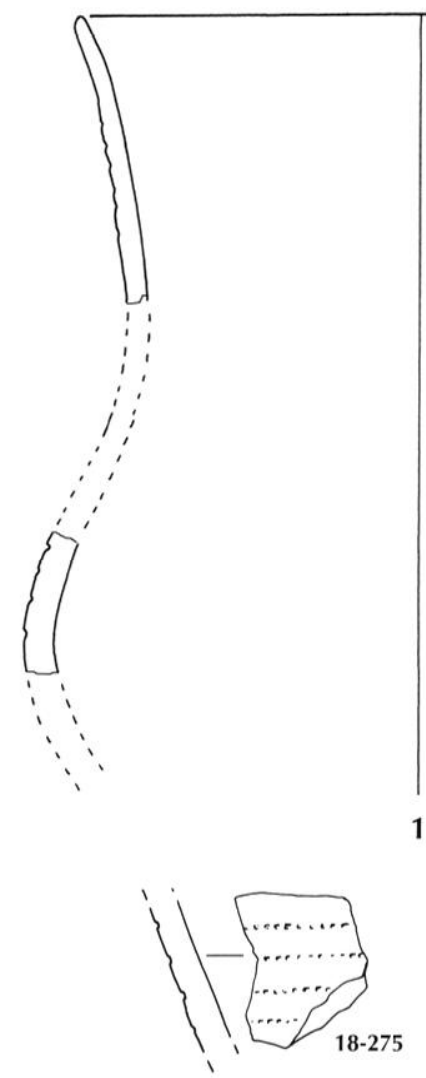

4

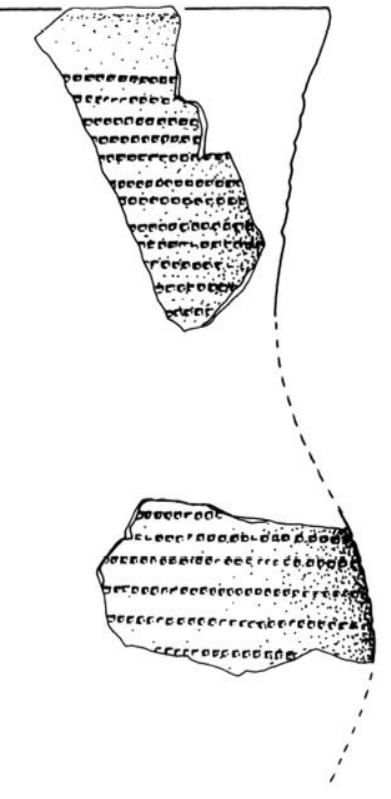

$18-279 / 280$
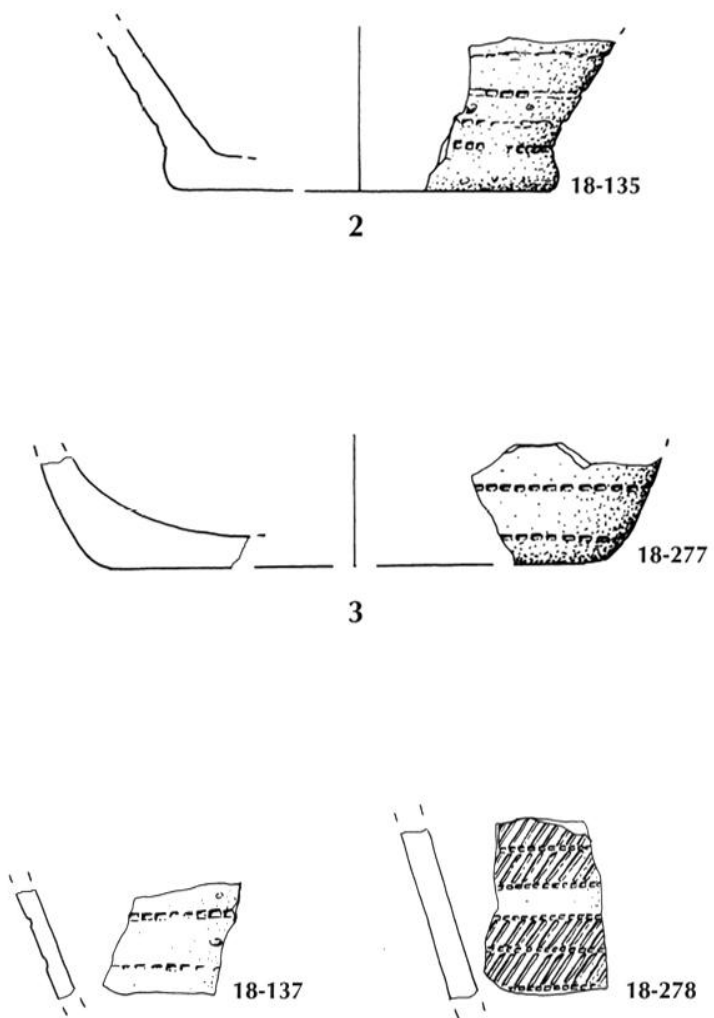

6

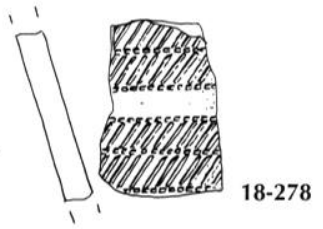

7

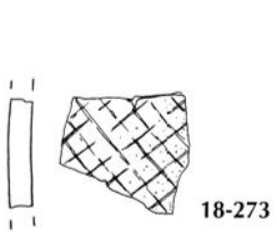

8

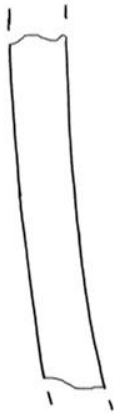

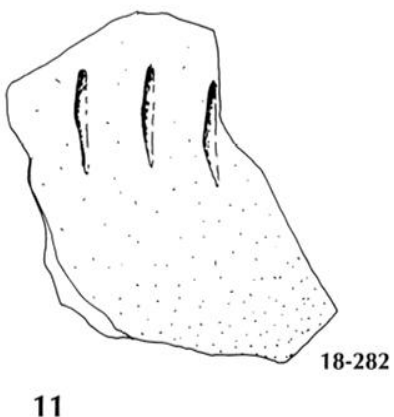
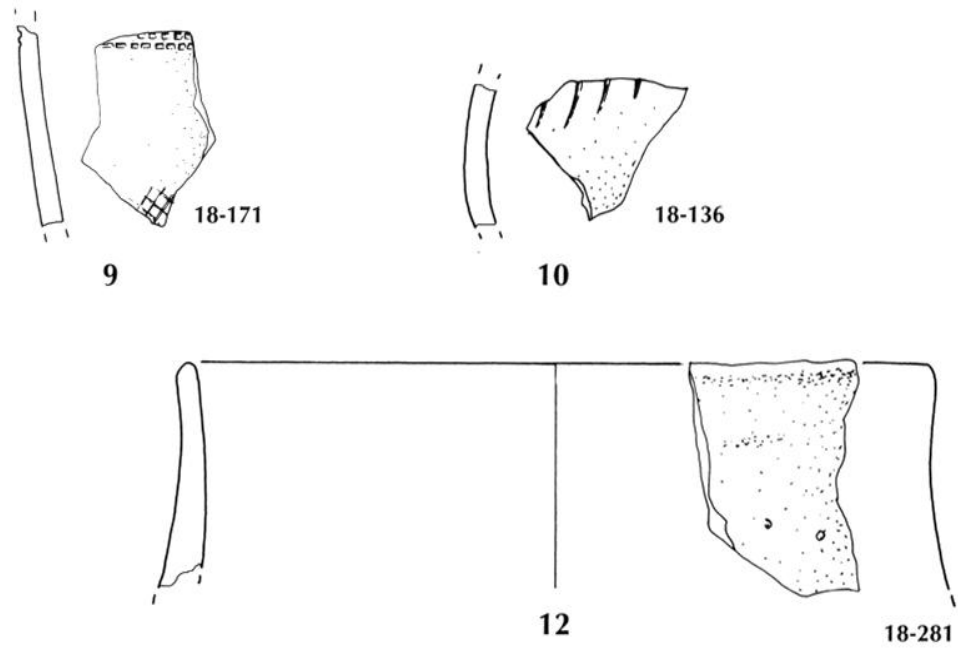

10

18-281 $5 \mathrm{~cm}$

Fig. 16-1-12: céramique décorée et non décorée du locus 1 , UF 18 (dessin F. Ducreux et F. Gauchet). 


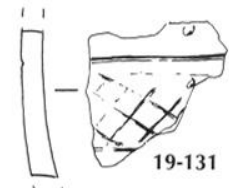

1

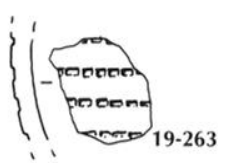

7

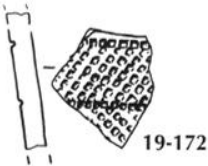

2

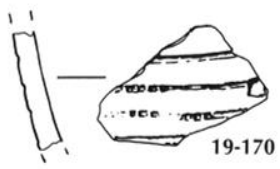

8

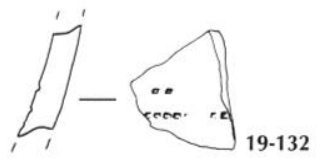

3

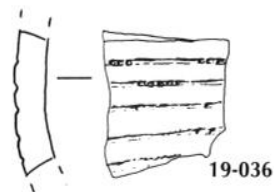

9

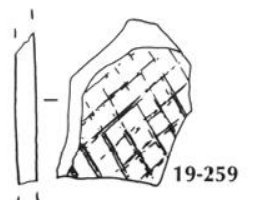

4

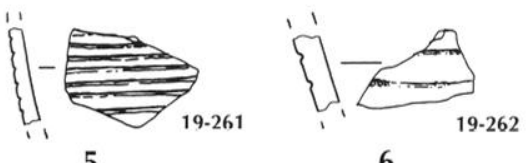

5
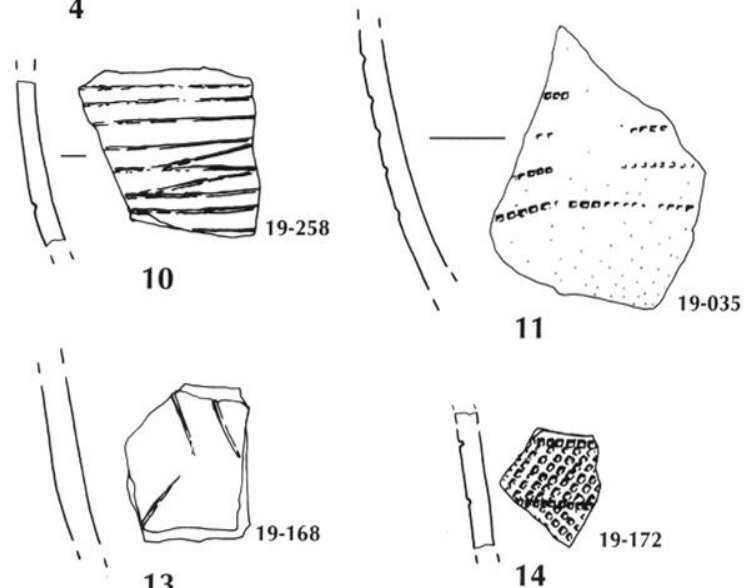

13

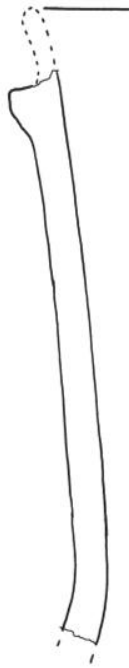

18

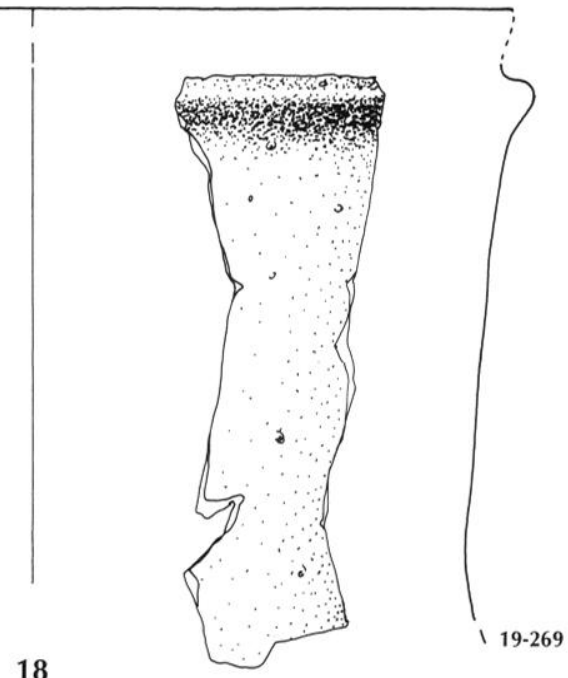

I 19-269

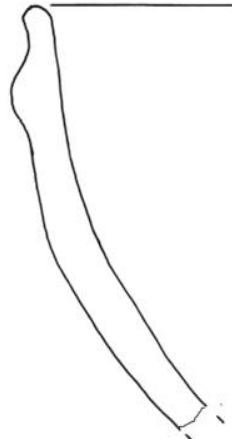

$5 \mathrm{~cm}$

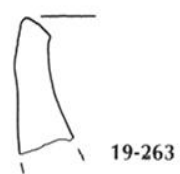

17

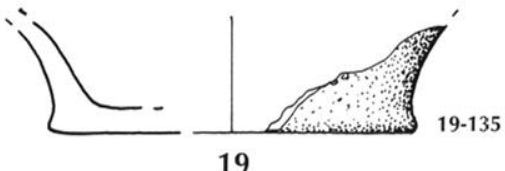

0 $5 \mathrm{~cm}$

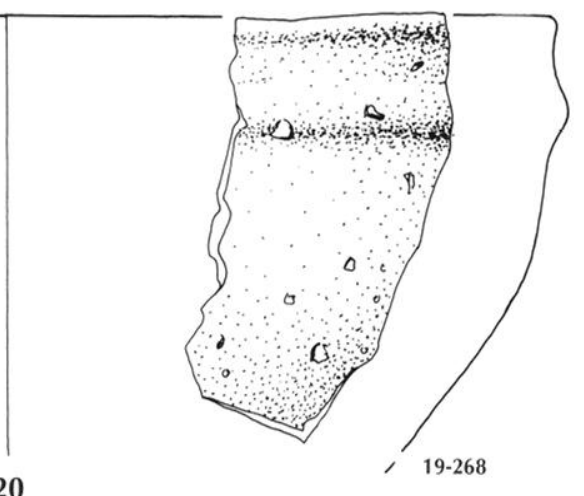

20

Fig. 17 - 1-20: céramique décorée et non décorée du locus 1, UF 19 (dessin F. Ducreux et F. Gauchet). 

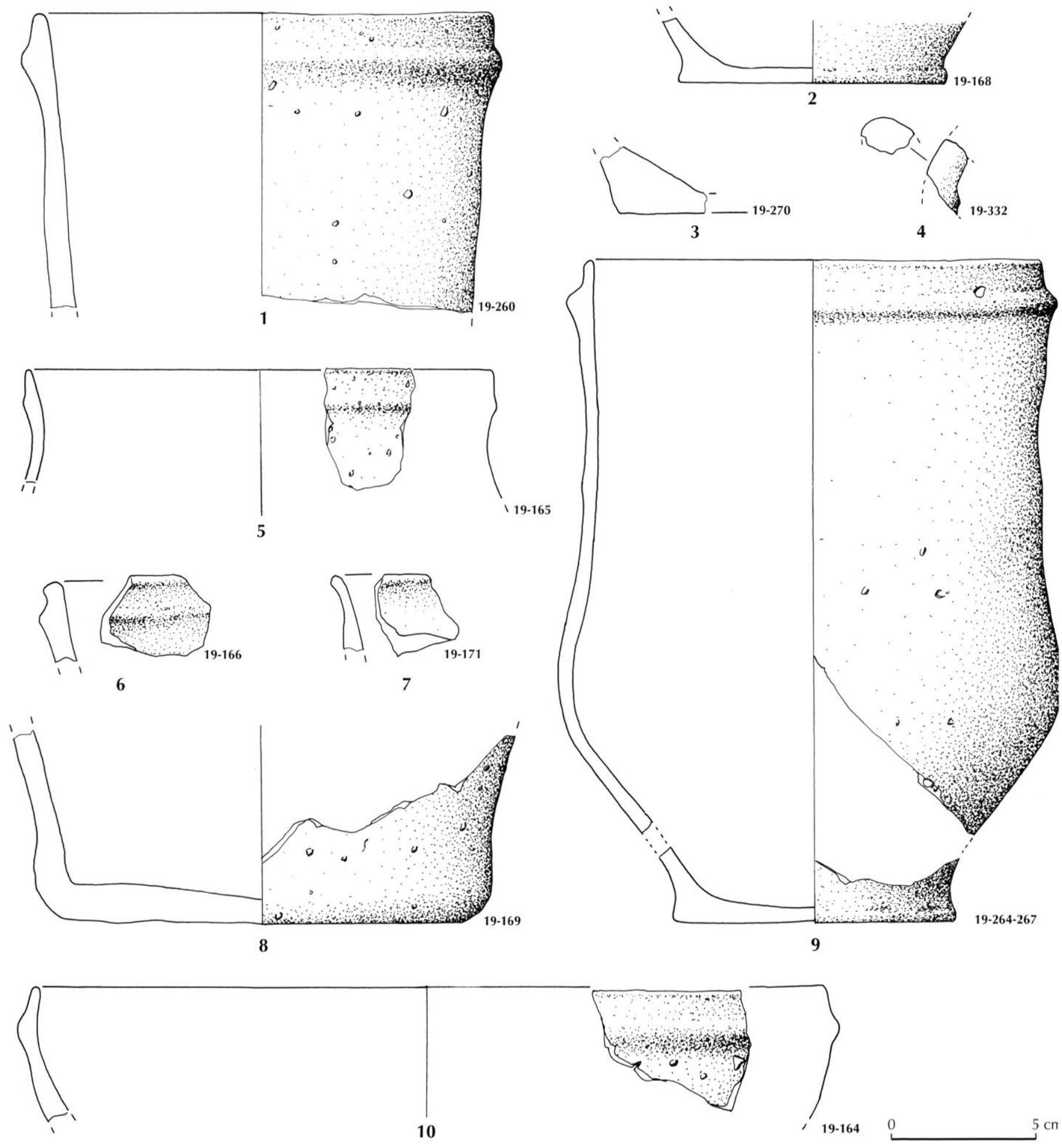

Fig. 18 - 1-10: céramique non décorée du locus 1, UF 19 (dessin F. Ducreux et F. Gauchet). 

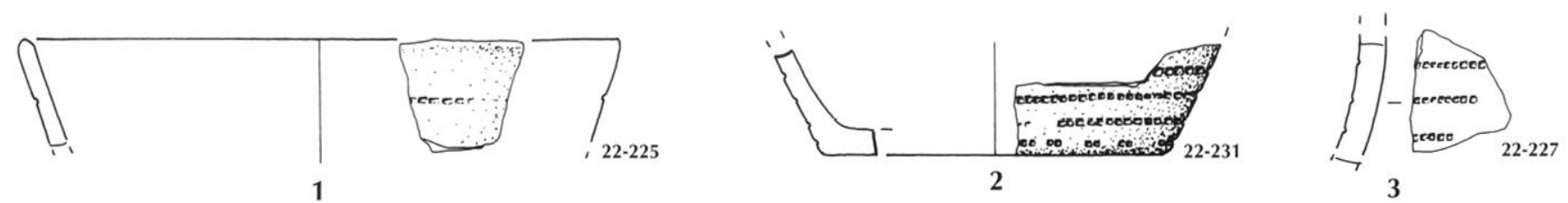

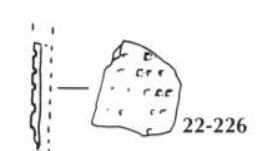

4
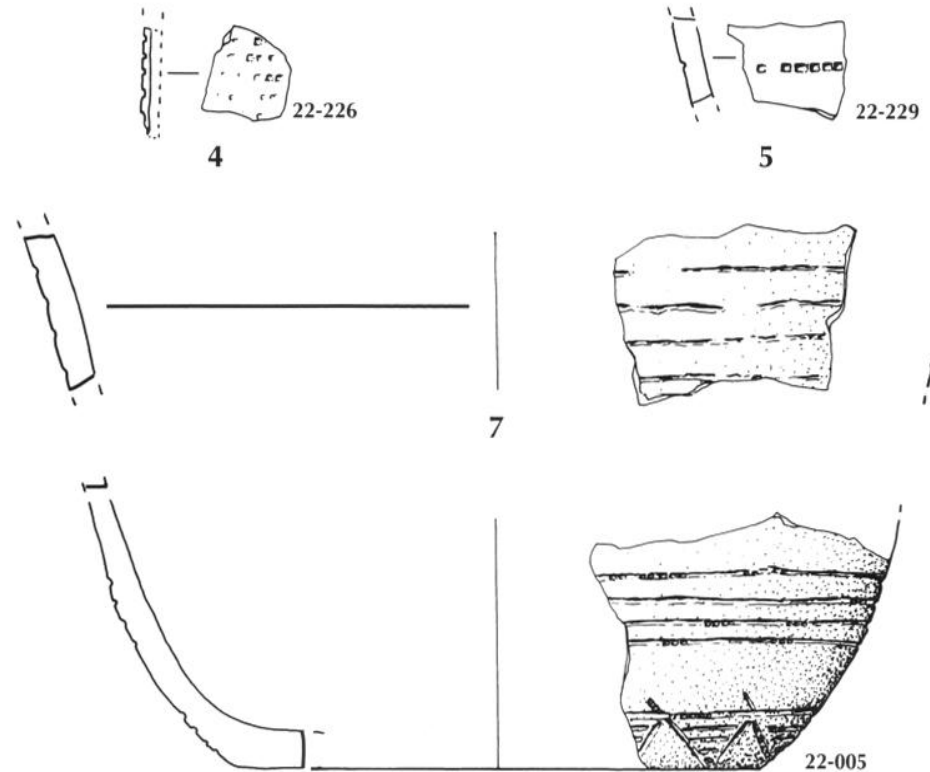

5
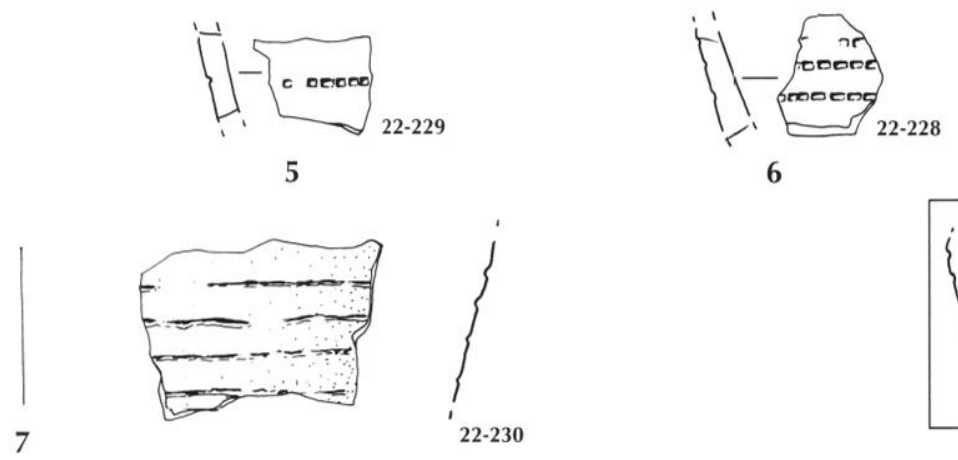

6
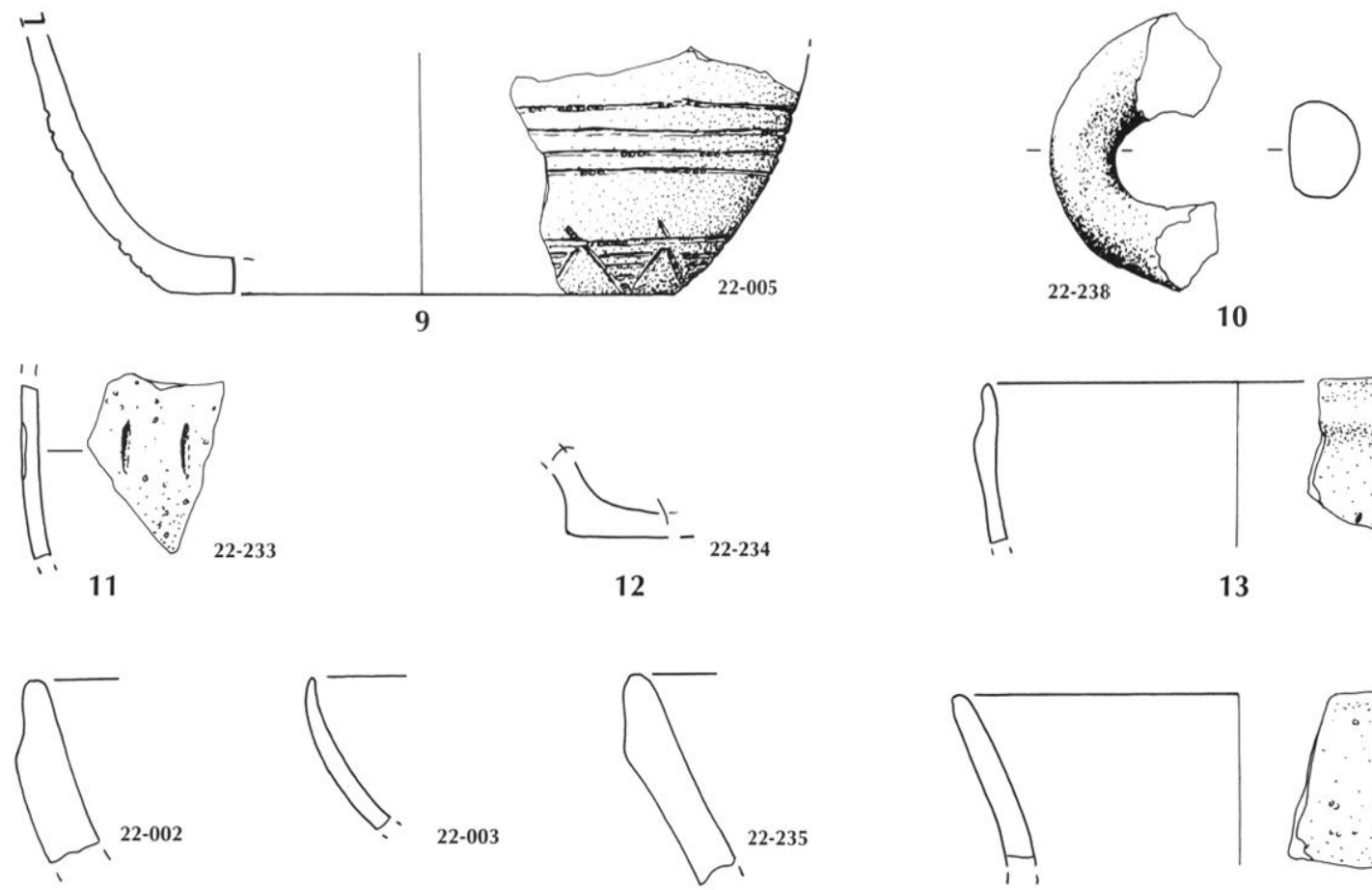

14
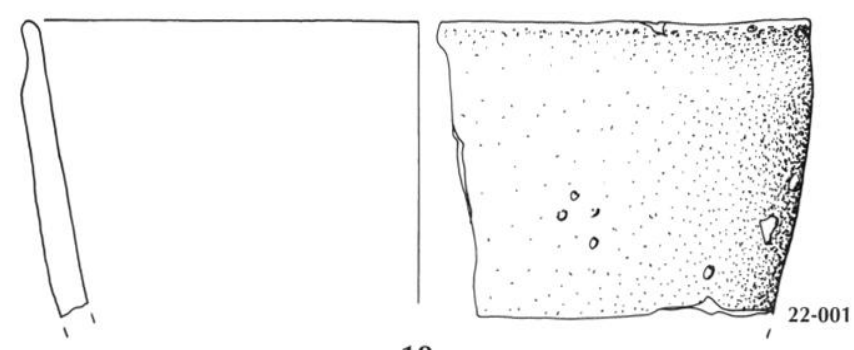

18

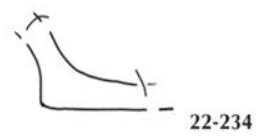

12

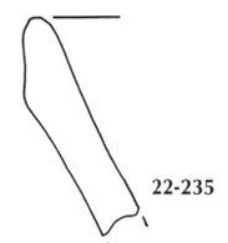

16

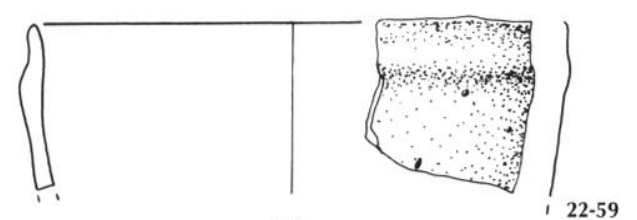

13

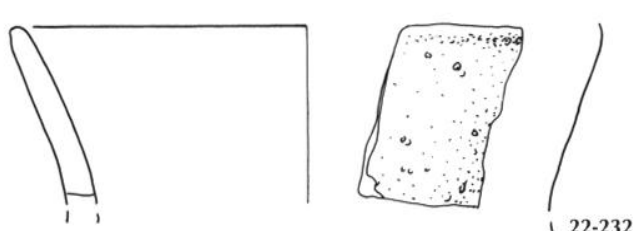

17

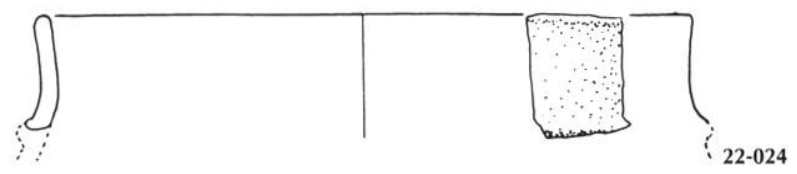

19

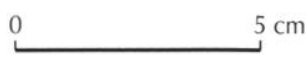

Fig. 19 - 1-19: céramique du locus 1, UF 22 et UF 26 (dessin F. Ducreux et F. Gauchet). 


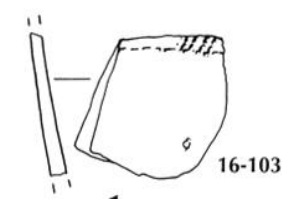

1

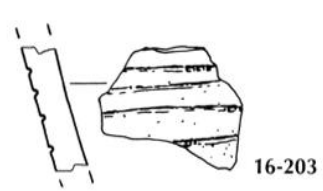

4

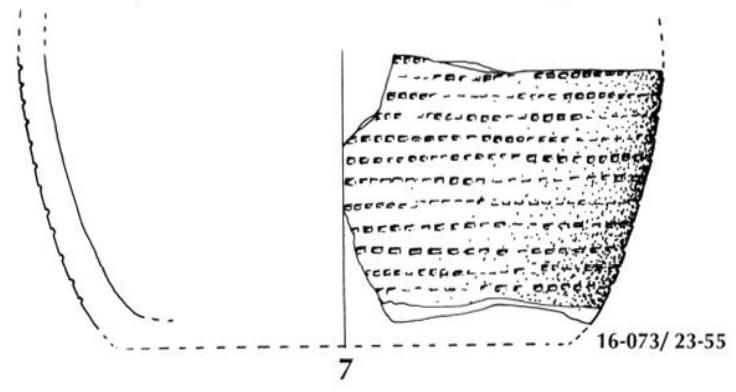

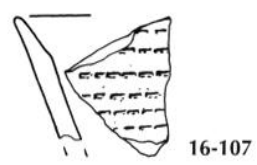

2

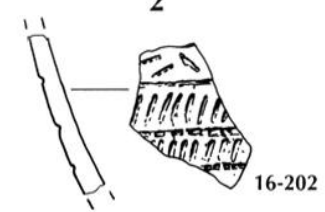

5
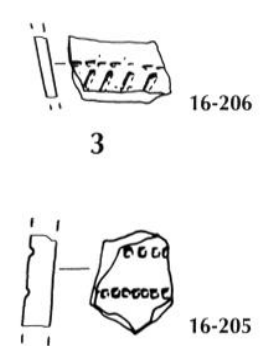

6

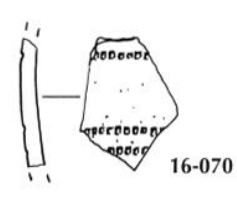

8

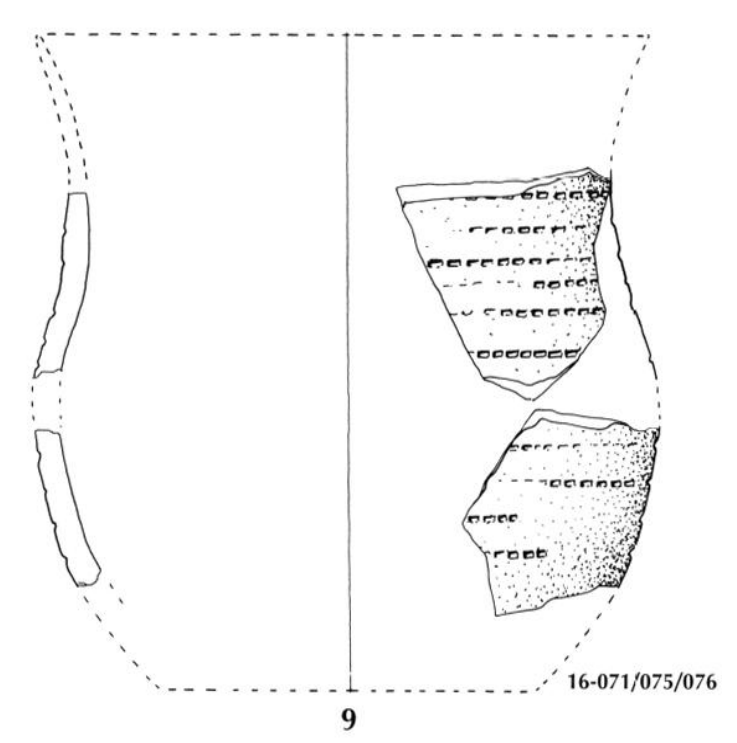

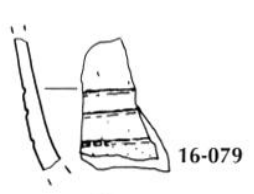

11

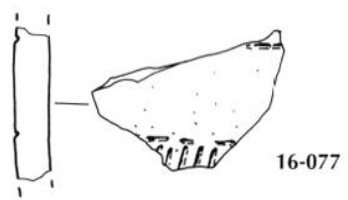

12
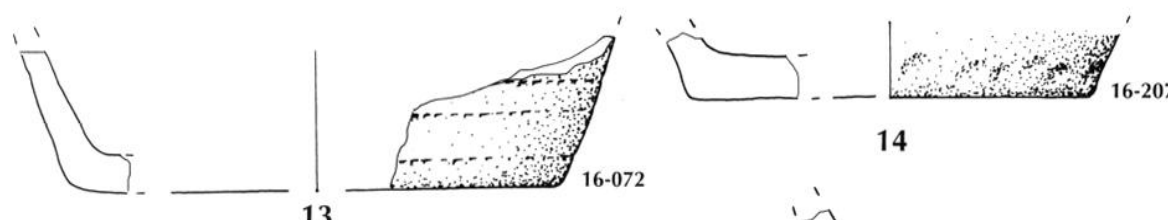

13
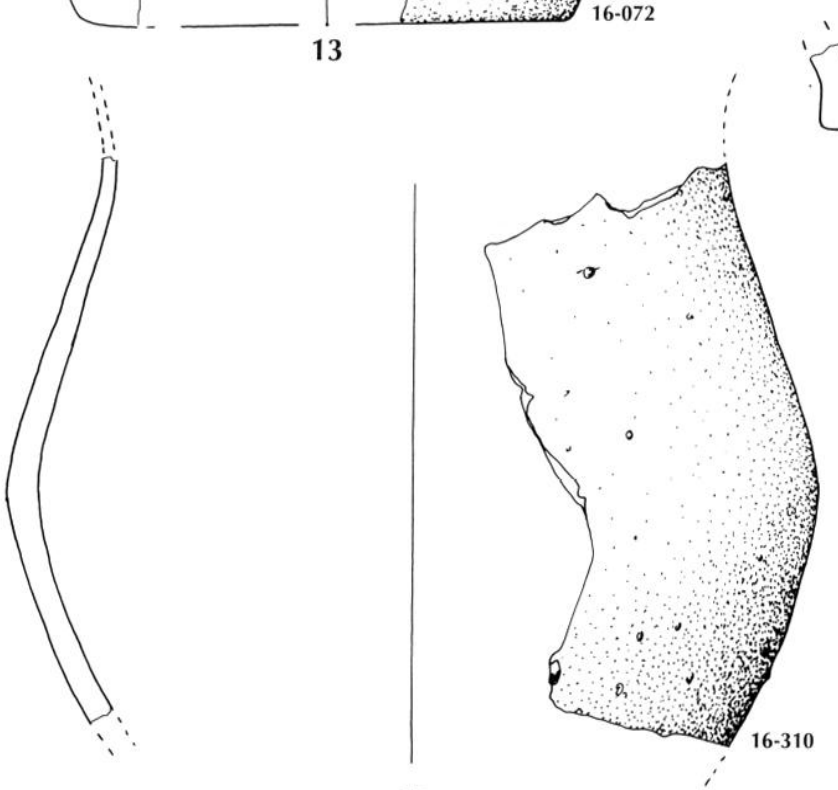

17

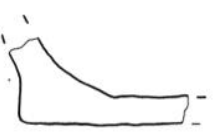

15
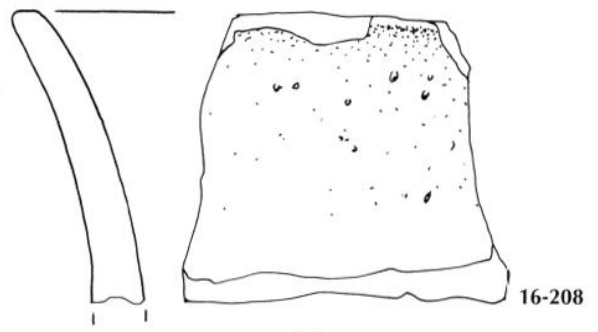

16

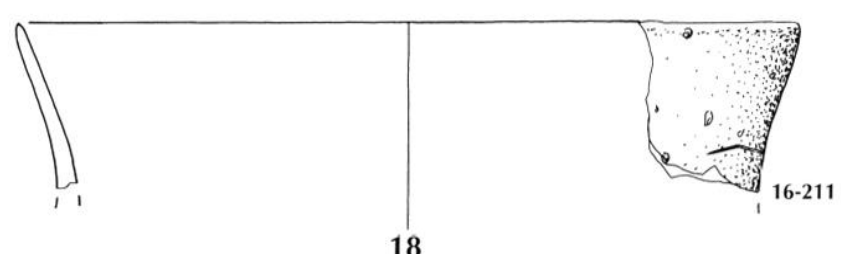

18
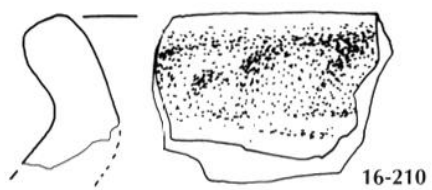

19

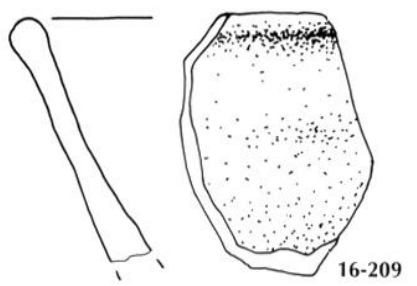

20

Fig. 20 - 1-20: céramique non décorée et décorée du locus 2, UF 16 (dessin F. Ducreux et F. Gauchet). 


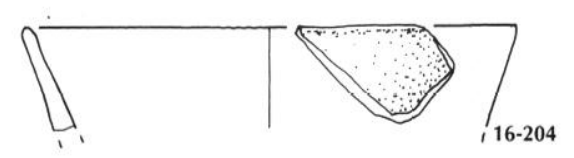

1

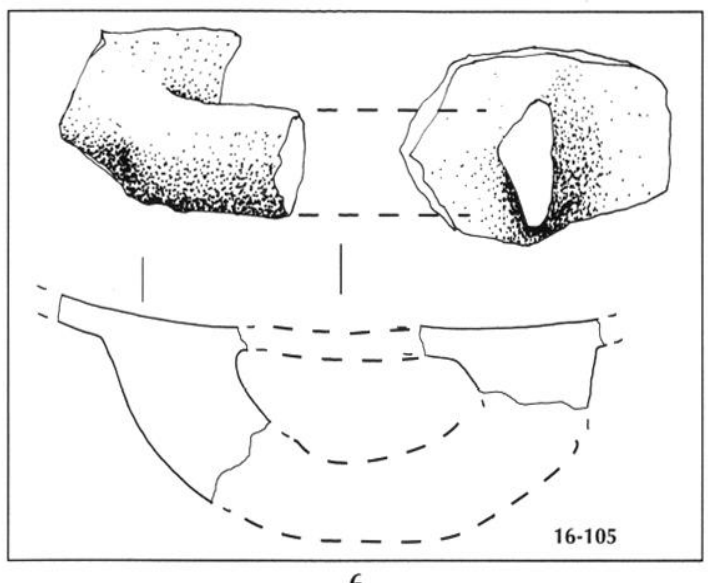

6

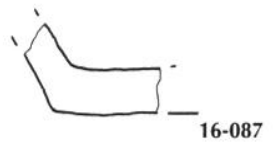

2

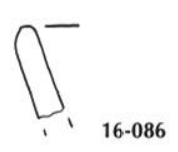

3

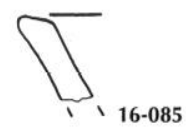

4
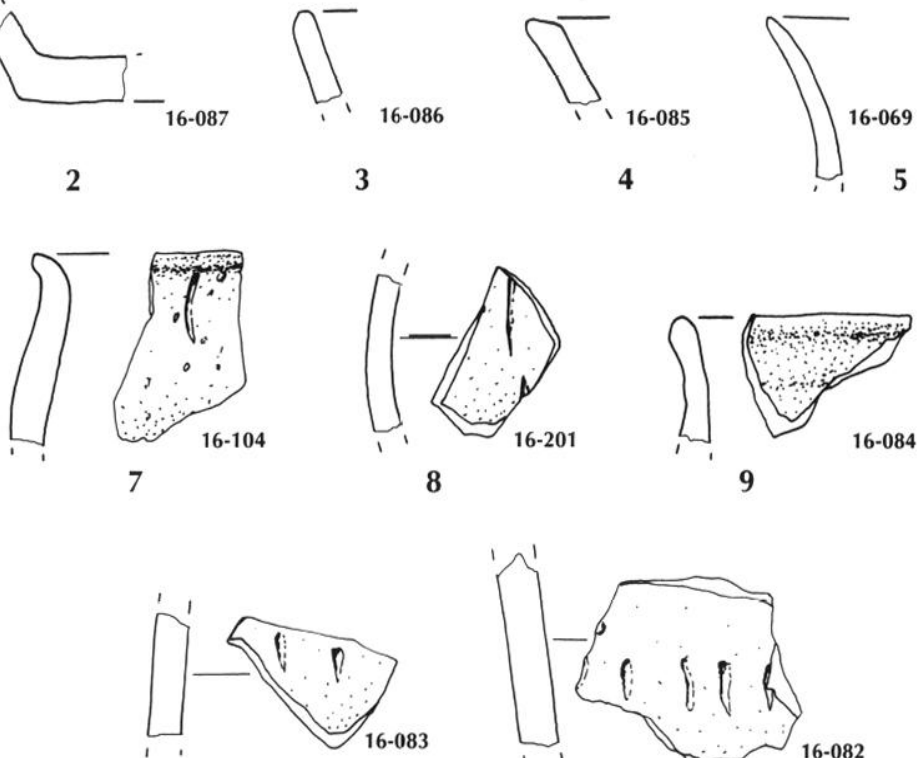

10

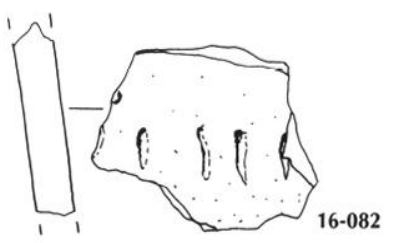

11
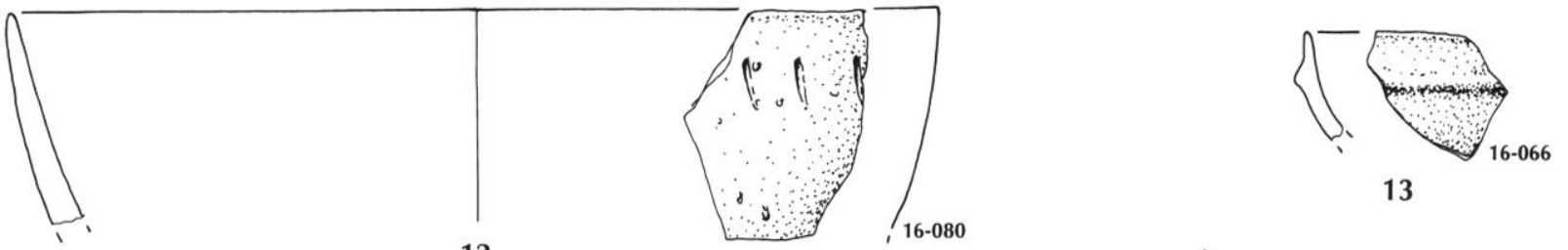

13

12
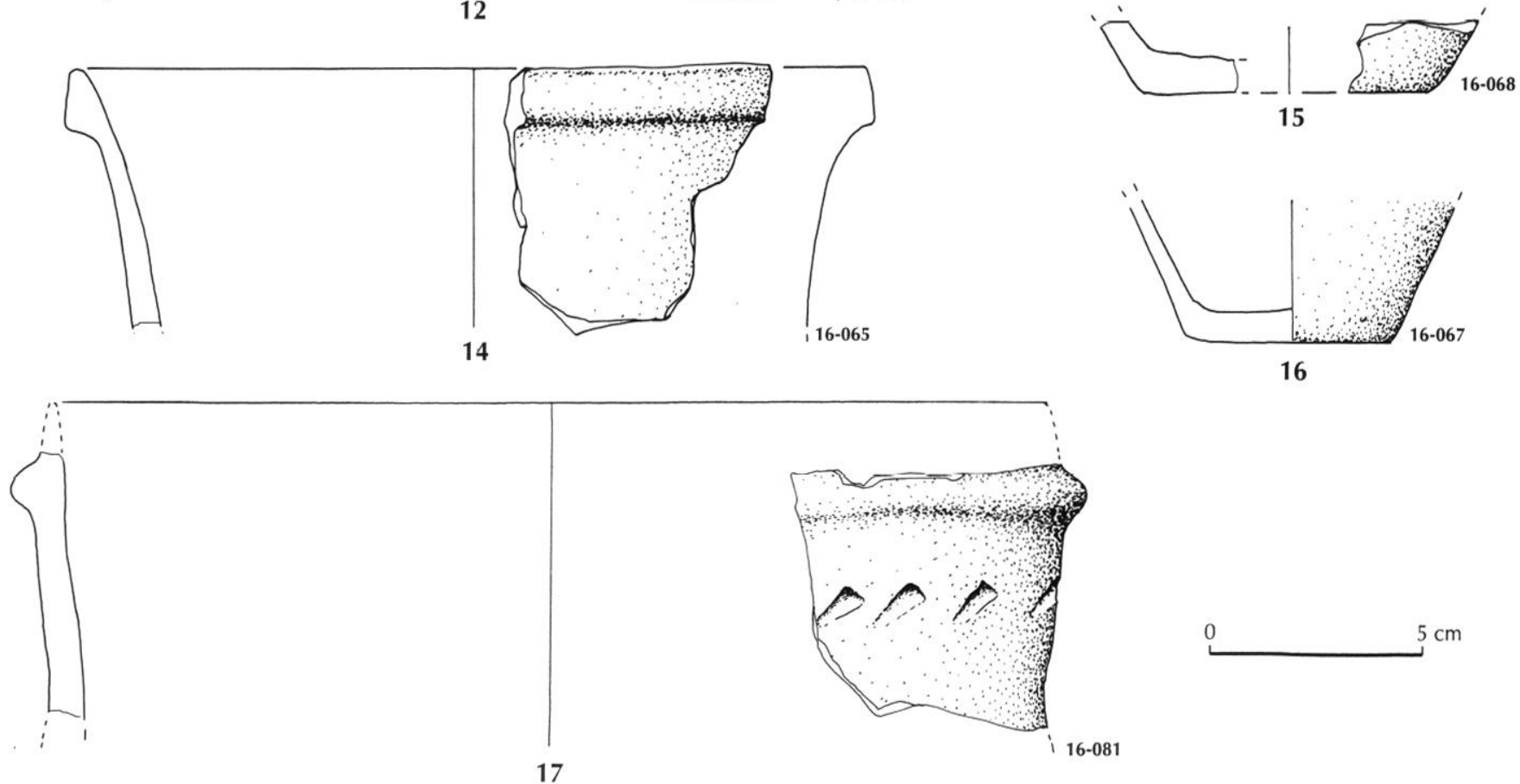

Fig. 21 - 1-17 : céramique commune du locus 2, UF 16 (dessin F. Ducreux et F. Gauchet). 

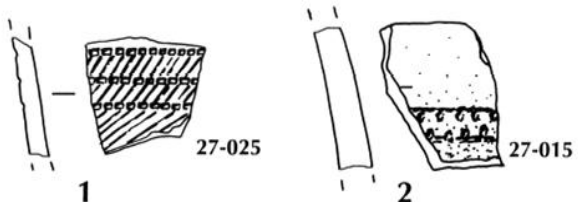

2
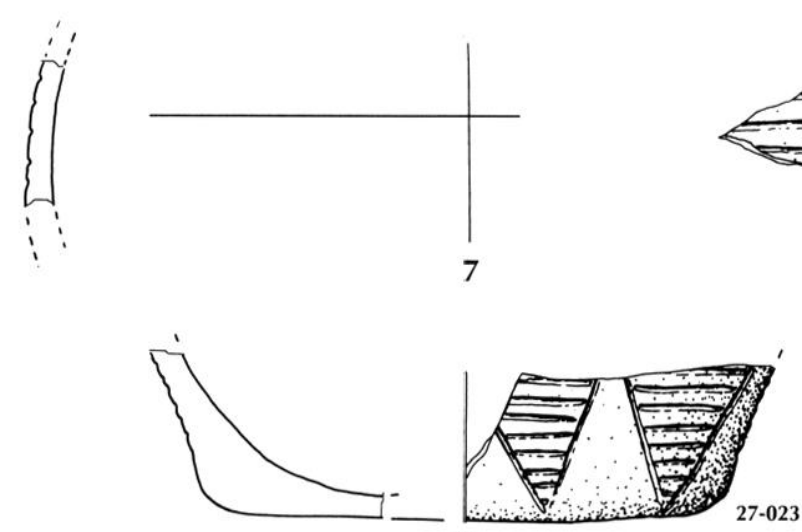

10

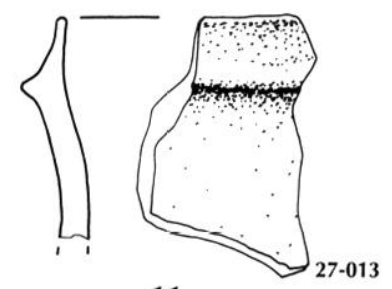

11
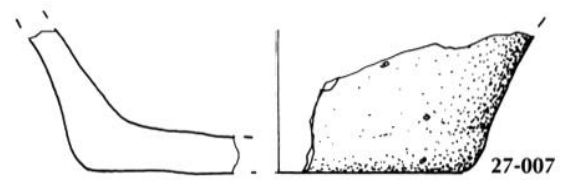

12
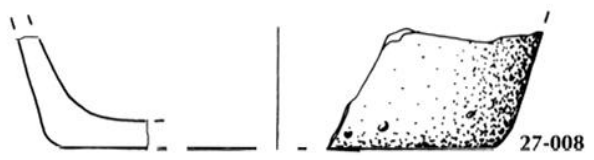

13
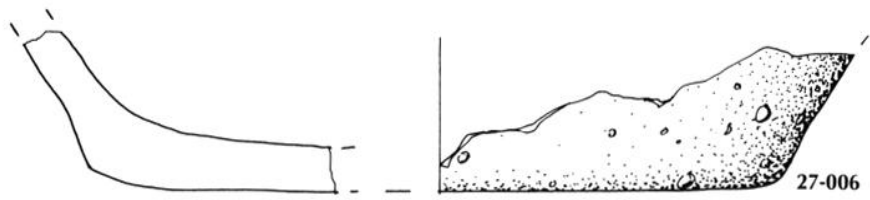

15
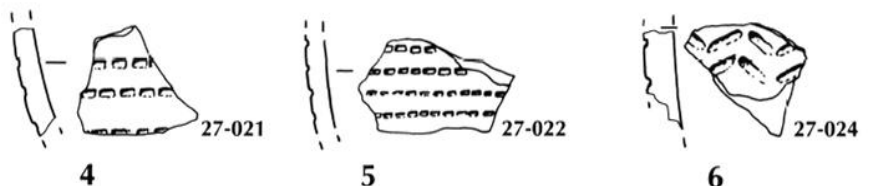

6

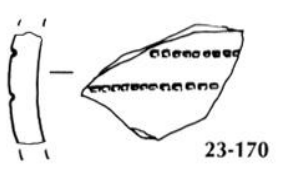

8

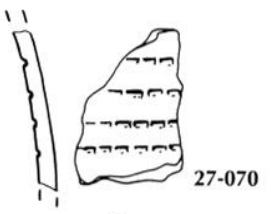

9

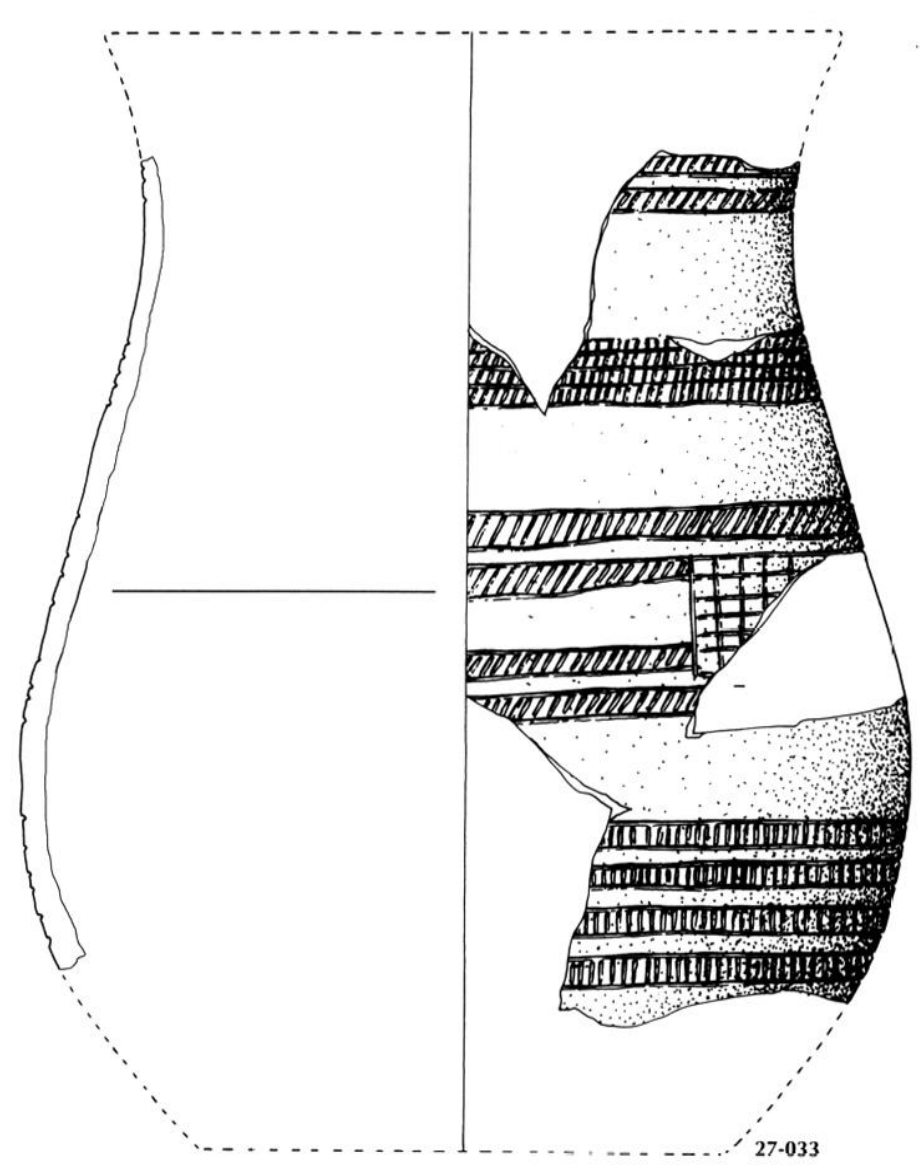

14

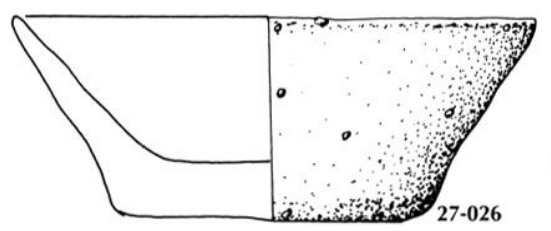

16

Fig. 22 - 1-16 : céramique du locus 2, UF 27 (dessin F. Ducreux et F. Gauchet). 

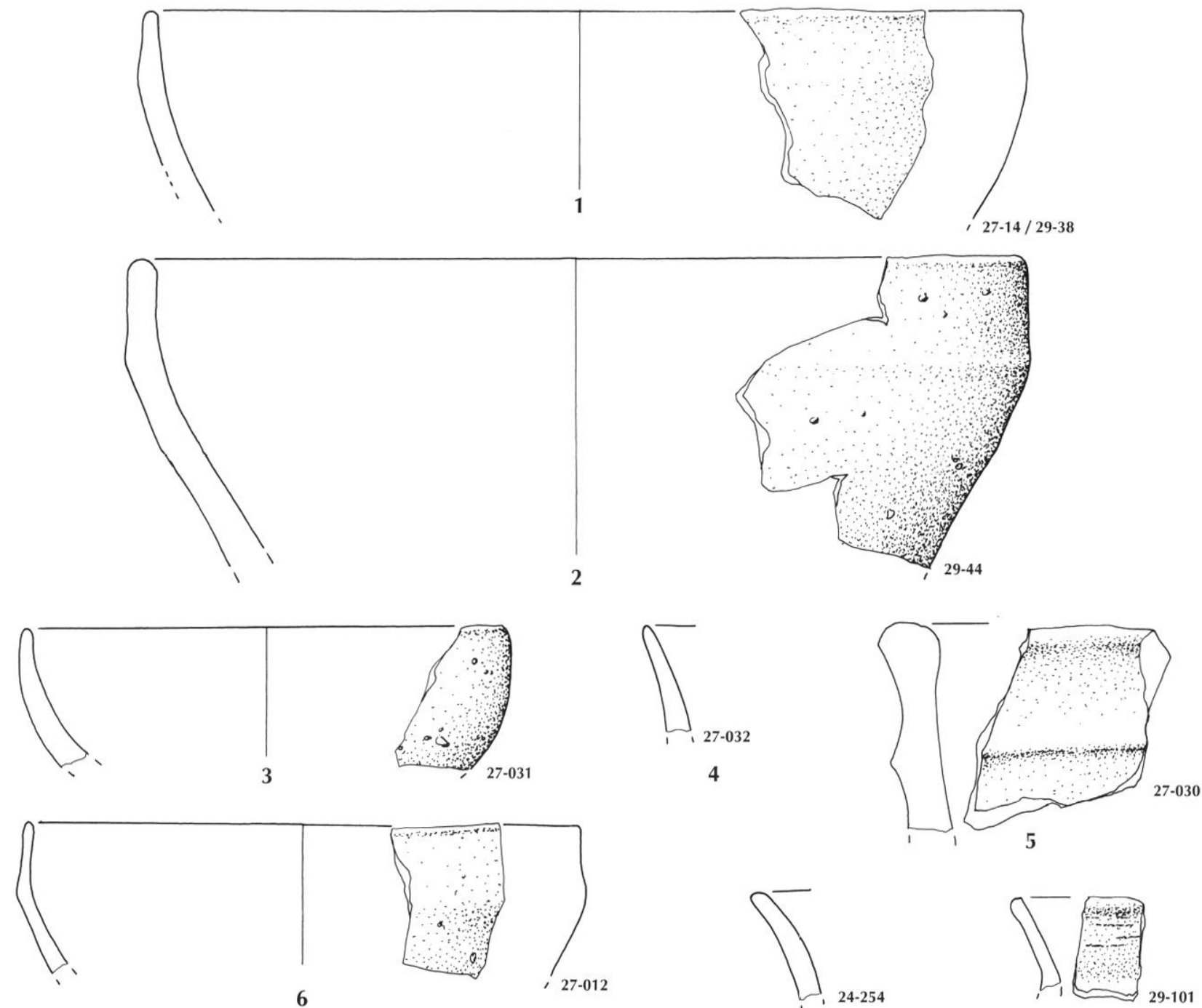

4

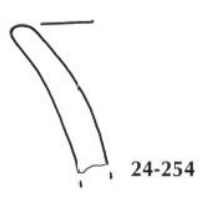

7

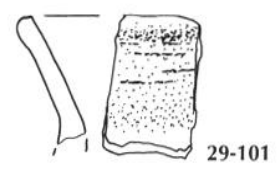

8
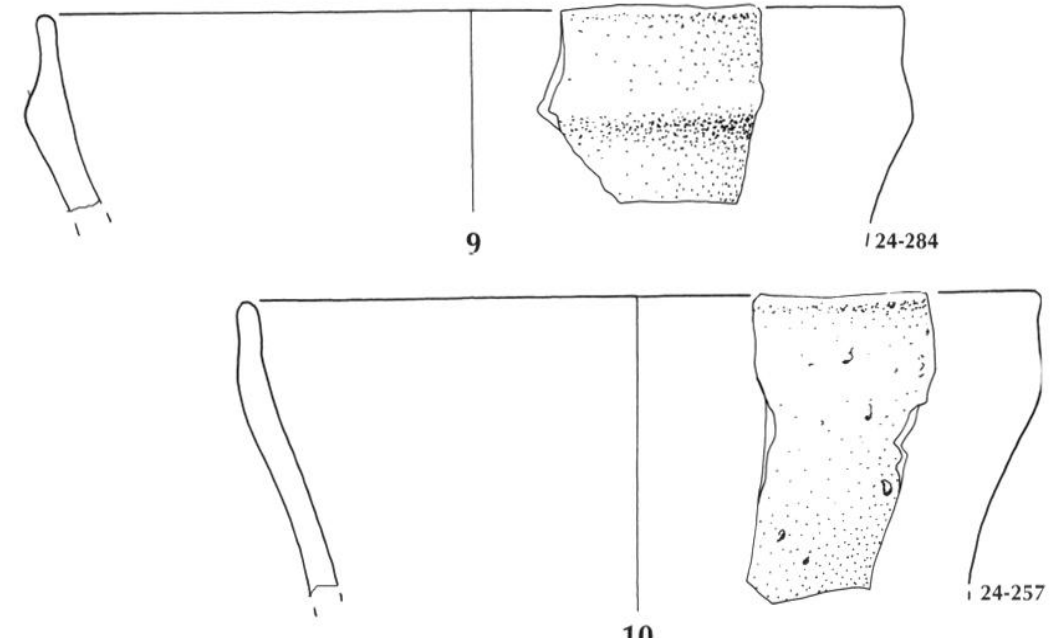

10

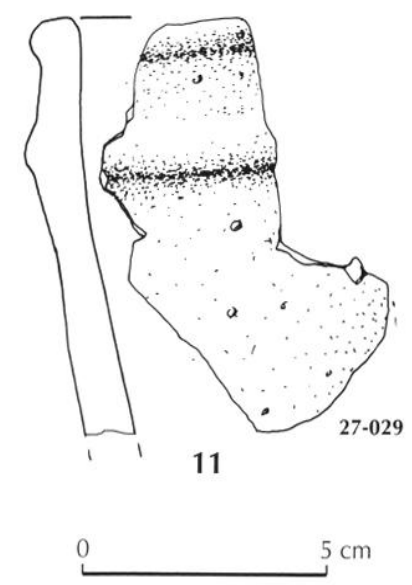

Fig. 23 - 1-11 : céramique non décorée du locus 2, UF 27, UF 29 et UF 24 (dessin F. Ducreux et F. Gauchet). 

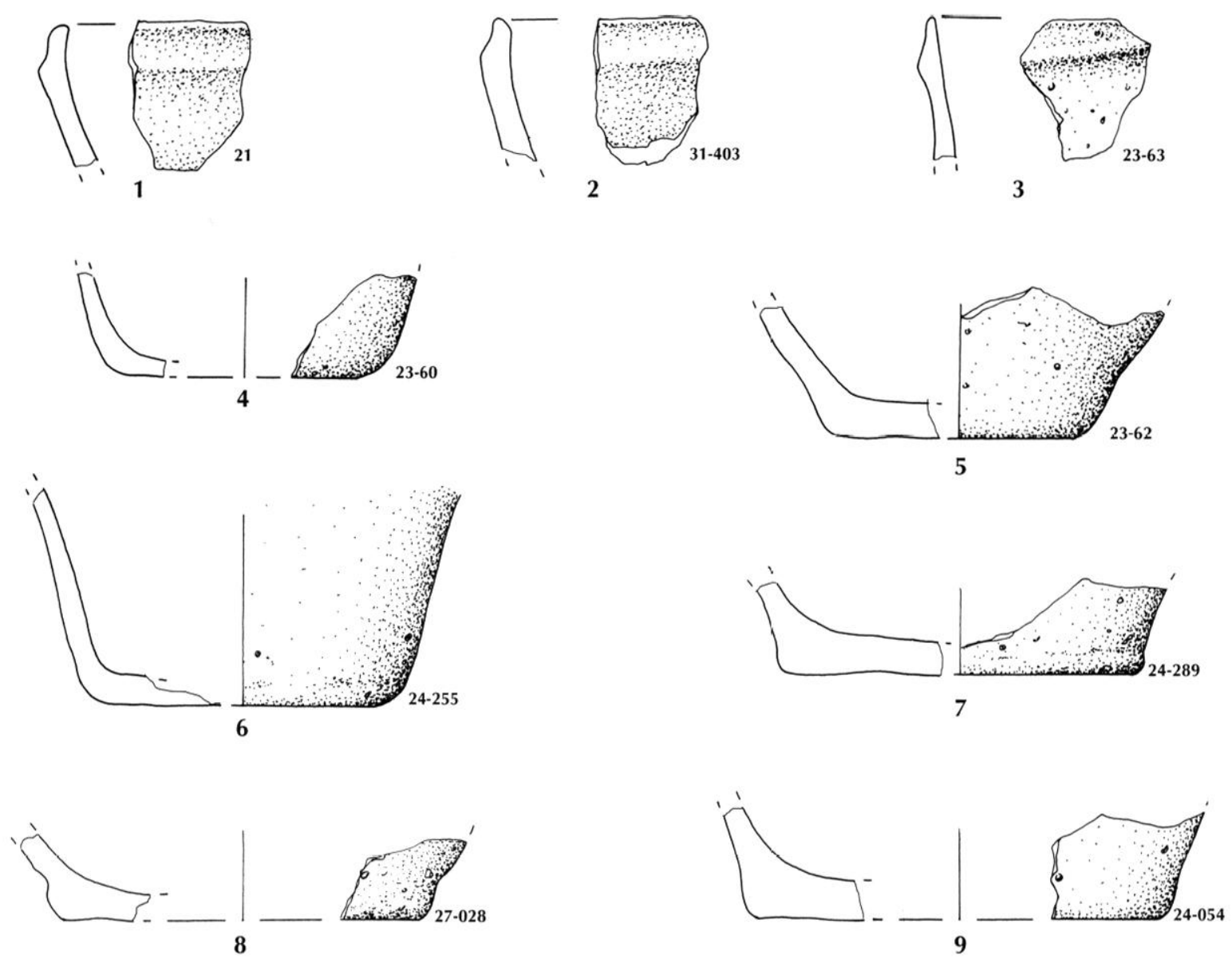

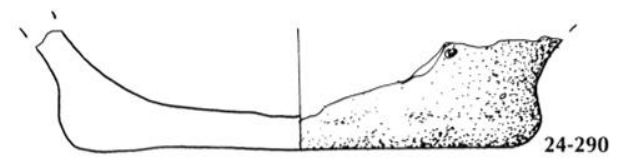

10
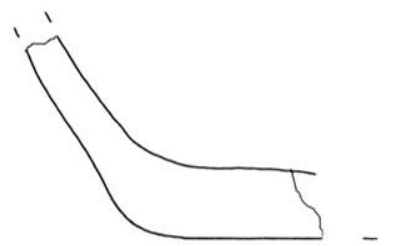

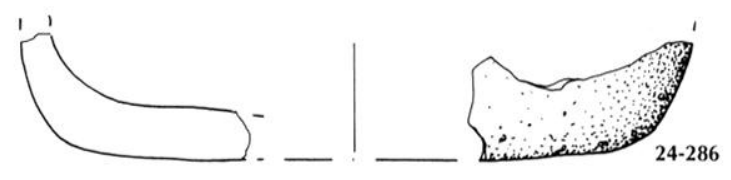

11
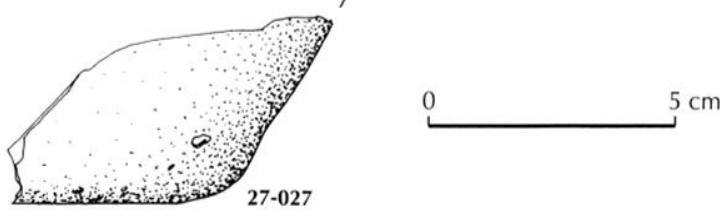

Fig. 24 - 1-12 : céramique non décorée du locus 2, UF 21, UF 23, UF 24, UF 27 et UF 31 (dessin F. Ducreux et F. Gauchet). 

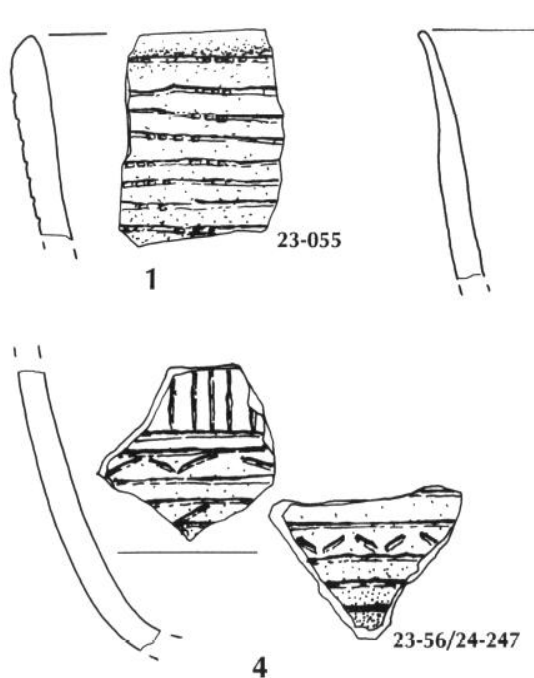

4
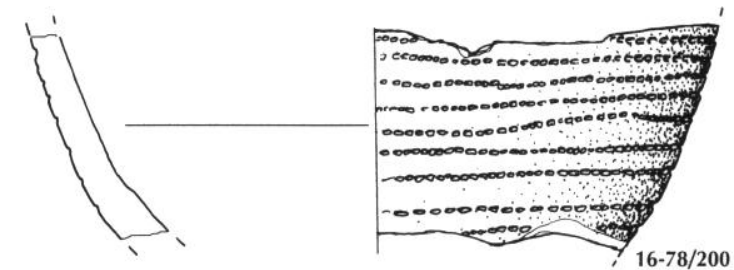

9
13

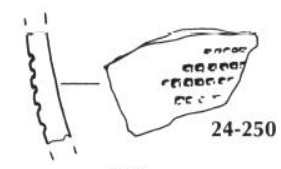

14

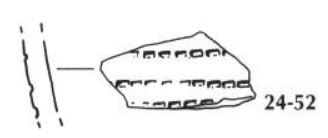

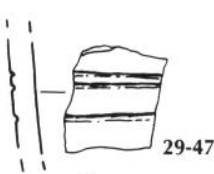

5
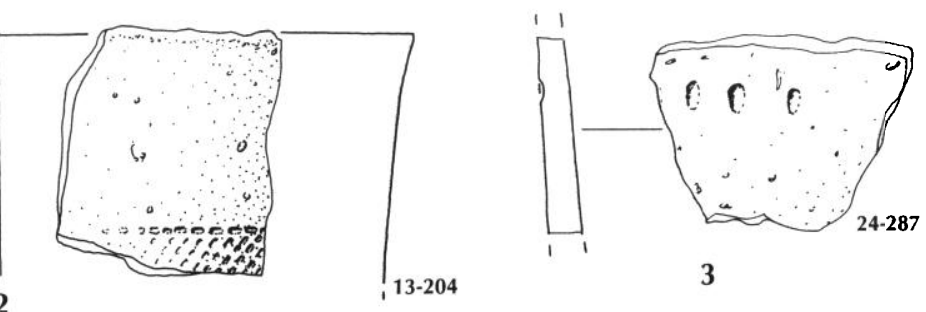

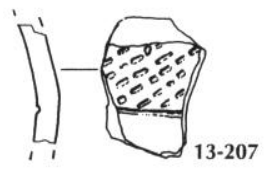

6

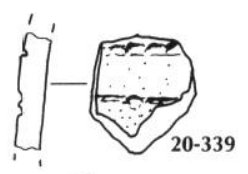

7

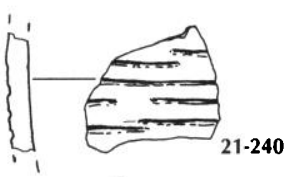

8

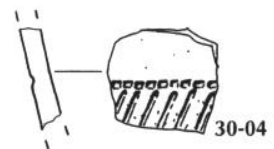

10

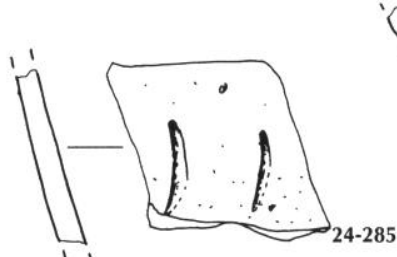

11

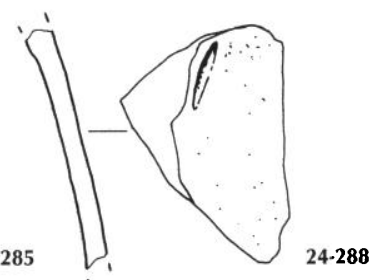

12

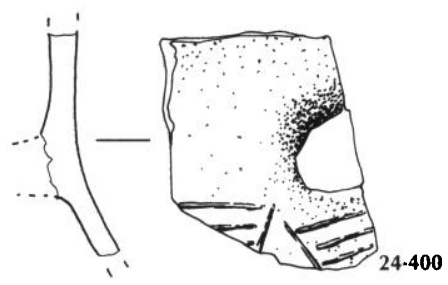

17

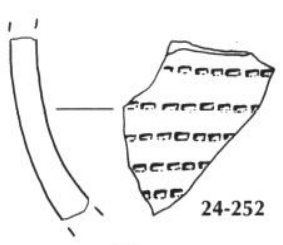

15

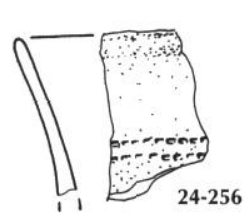

16

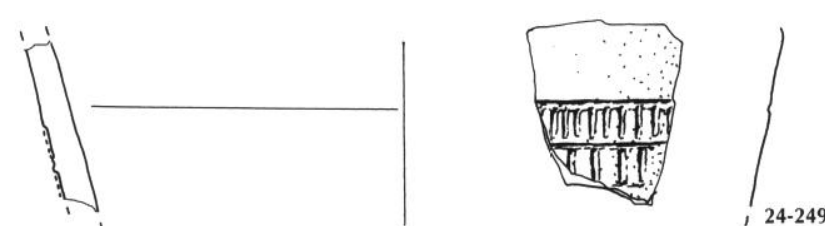

18

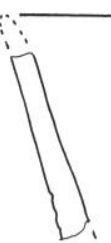

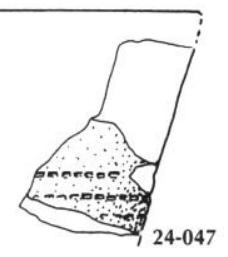

24-047

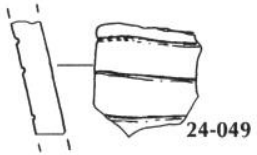

19

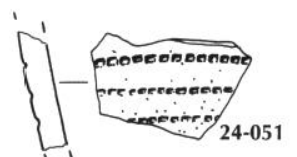

20

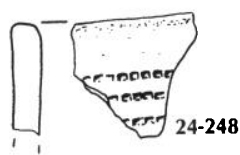

21
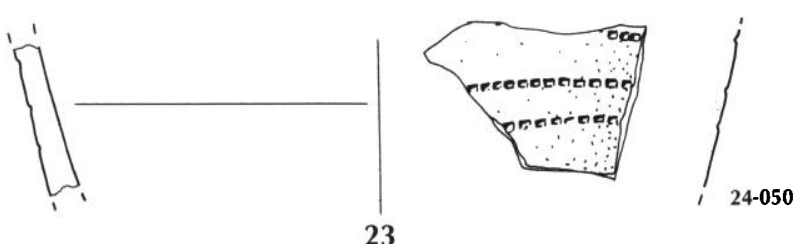

23 $5 \mathrm{~cm}$

Fig. 25 - 1-23 : céramique décorée du locus 2 (dessin F. Ducreux et F. Gauchet). 

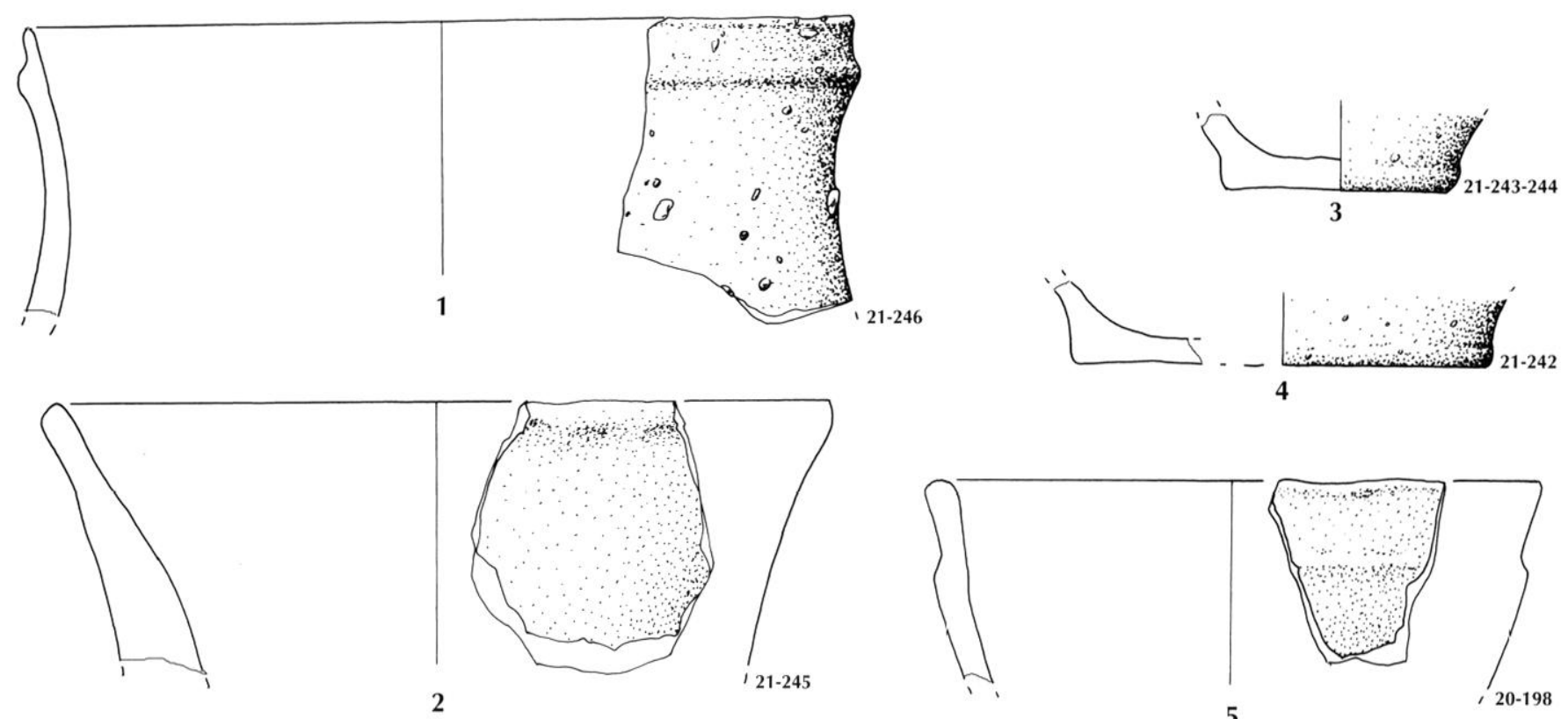

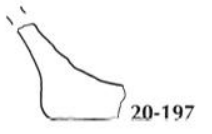

6

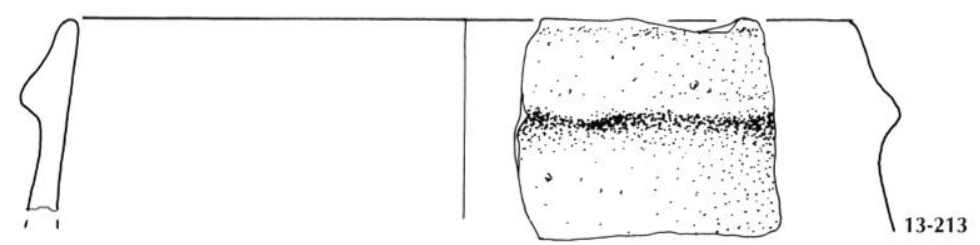

12

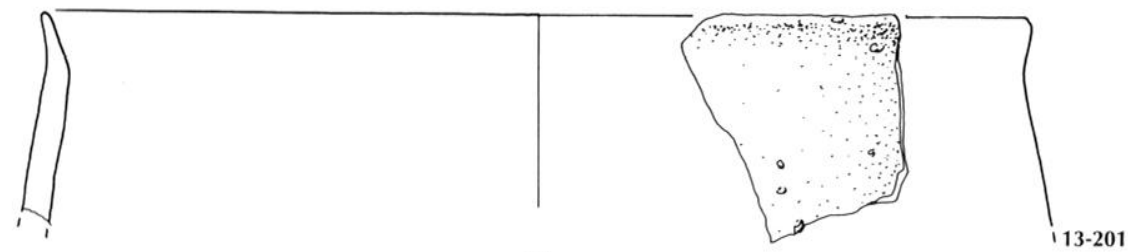

14

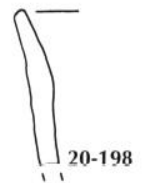

8

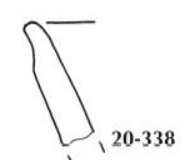

9

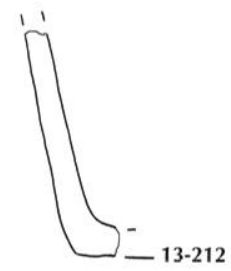

11

10

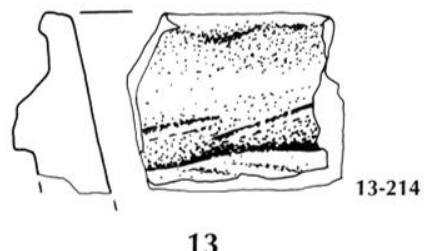

13

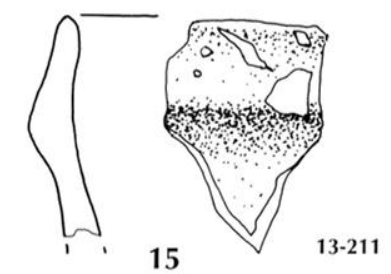

0 $5 \mathrm{~cm}$

Fig. 26 - 1-15: céramique non décorée et décorée du locus 2, UF 13, UF 20 et UF 21 (dessin F. Ducreux et F: Gauchet). 

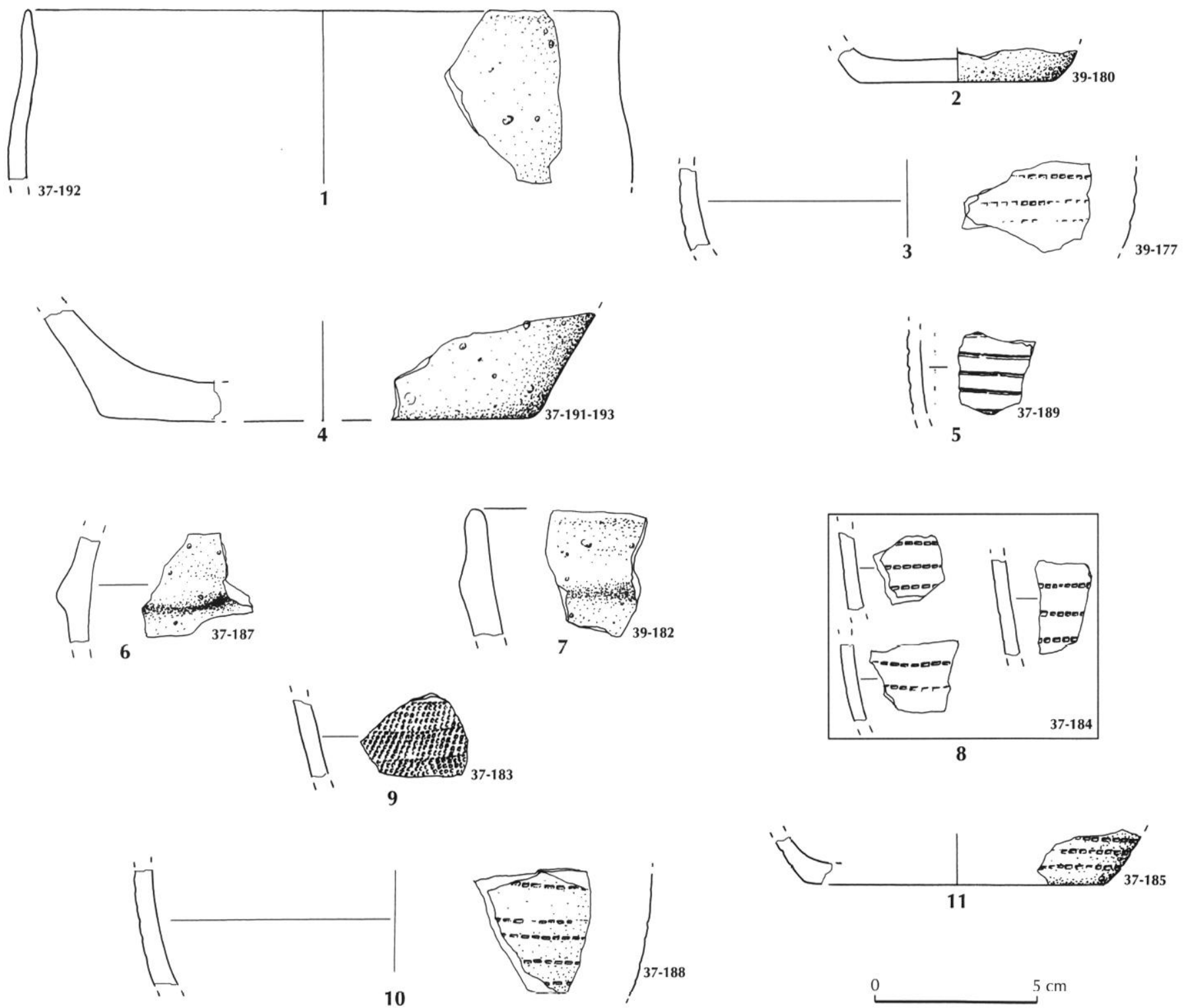

Fig. 27 - 1-11 : céramique non décorée et décorée du locus 3, UF 39 et UF 37 (dessin F. Ducreux et F. Gauchet). 

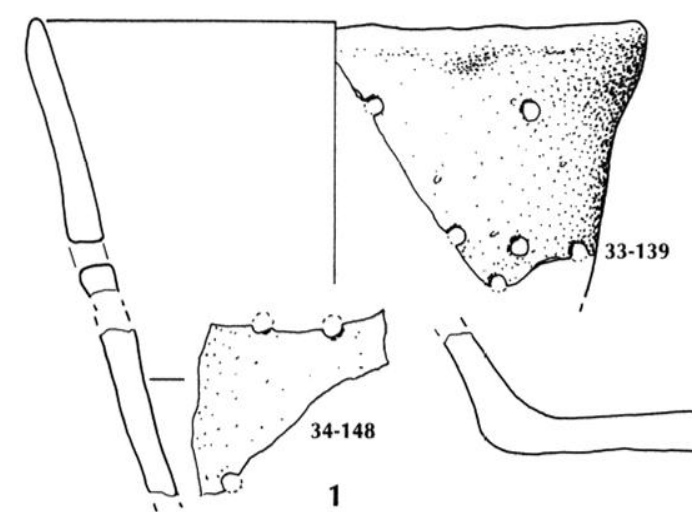

1
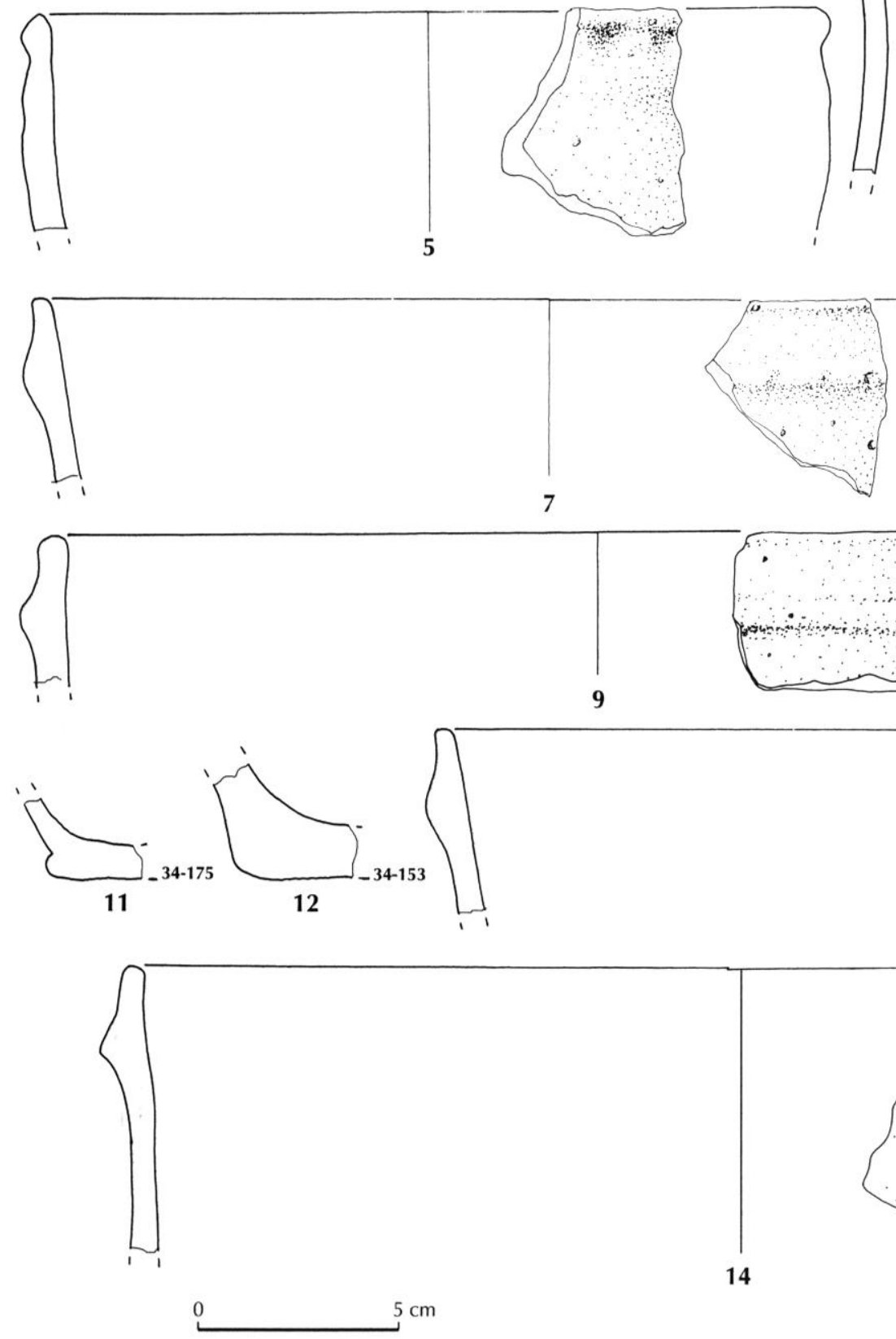

Fig. 28 - 1-14 : céramique non décorée et décorée du locus 4, UF 32, UF 33 et UF 34 (dessin F. Ducreux et F. Gauchet). 

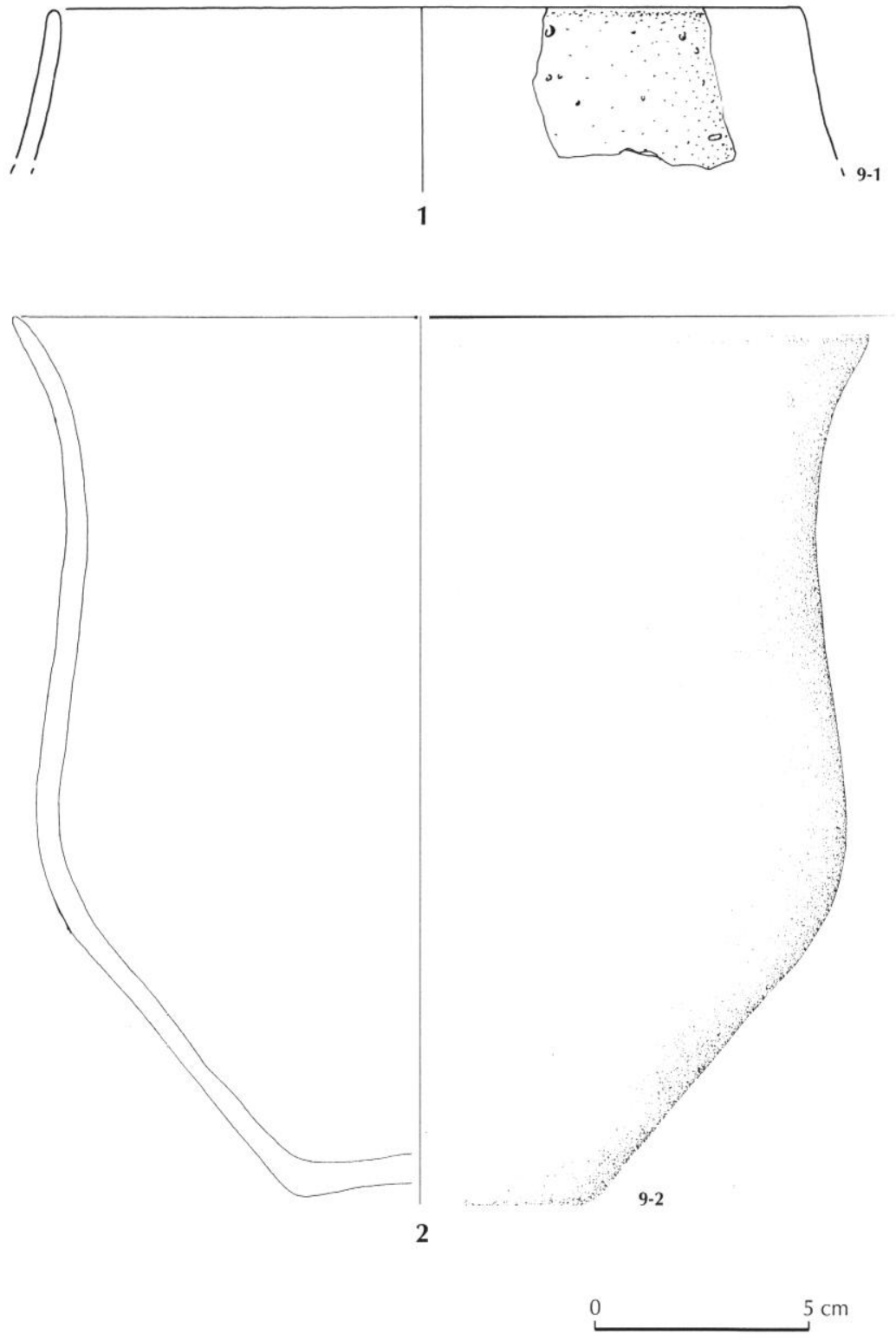

Fig. 29 - 1, 2 : céramique non décorée du locus 5, UF 9 (dessin F. Ducreux et F. Gauchet). 


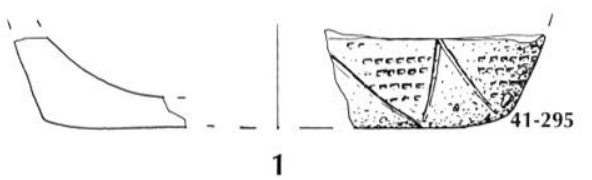

$\ddot{1}$
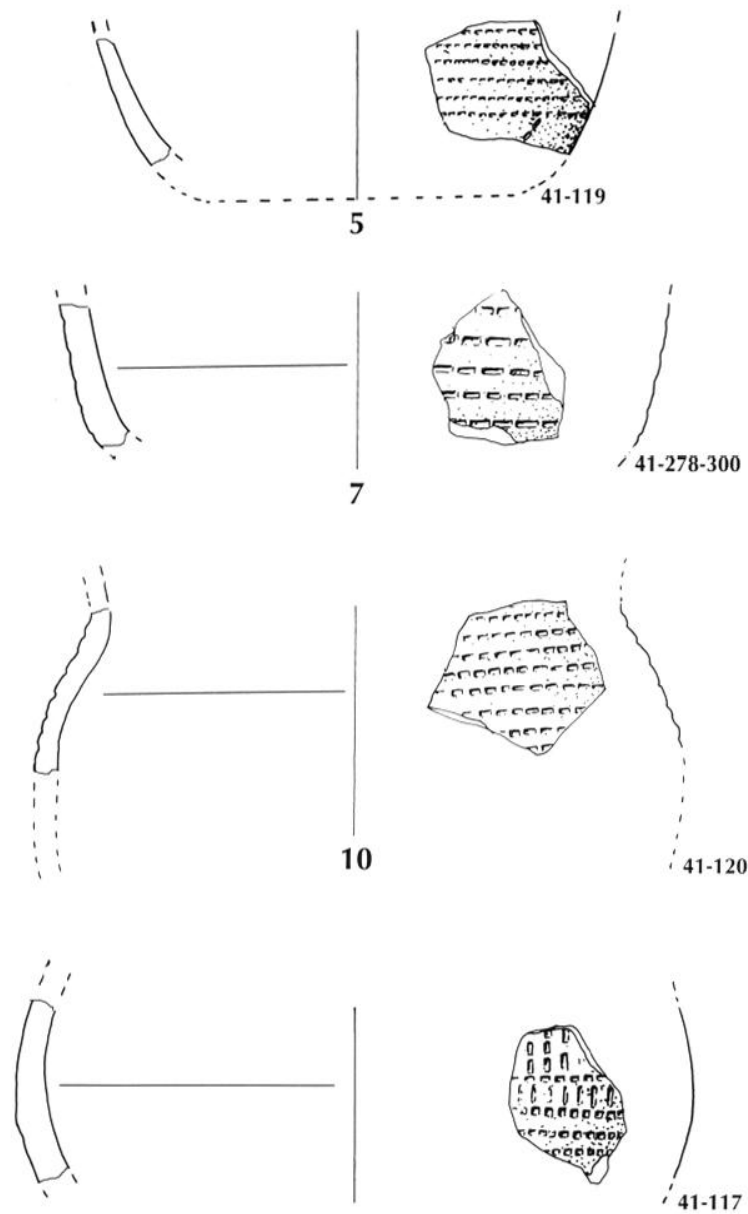

15
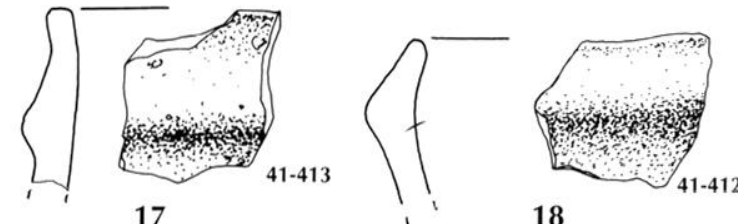

18
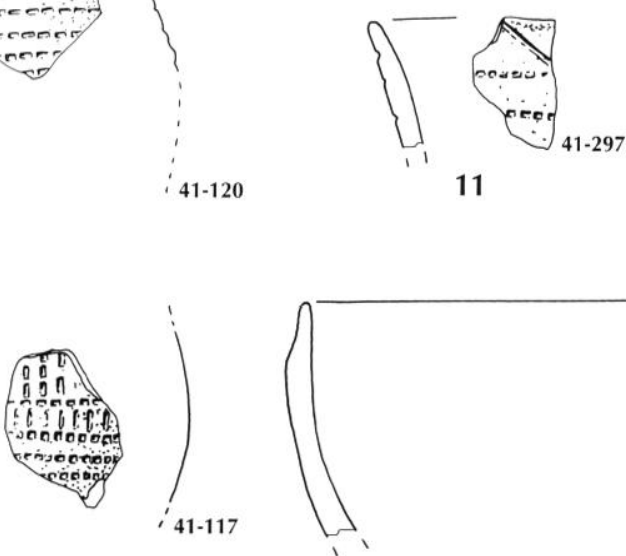

11

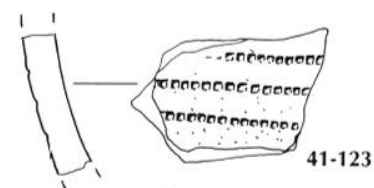

3
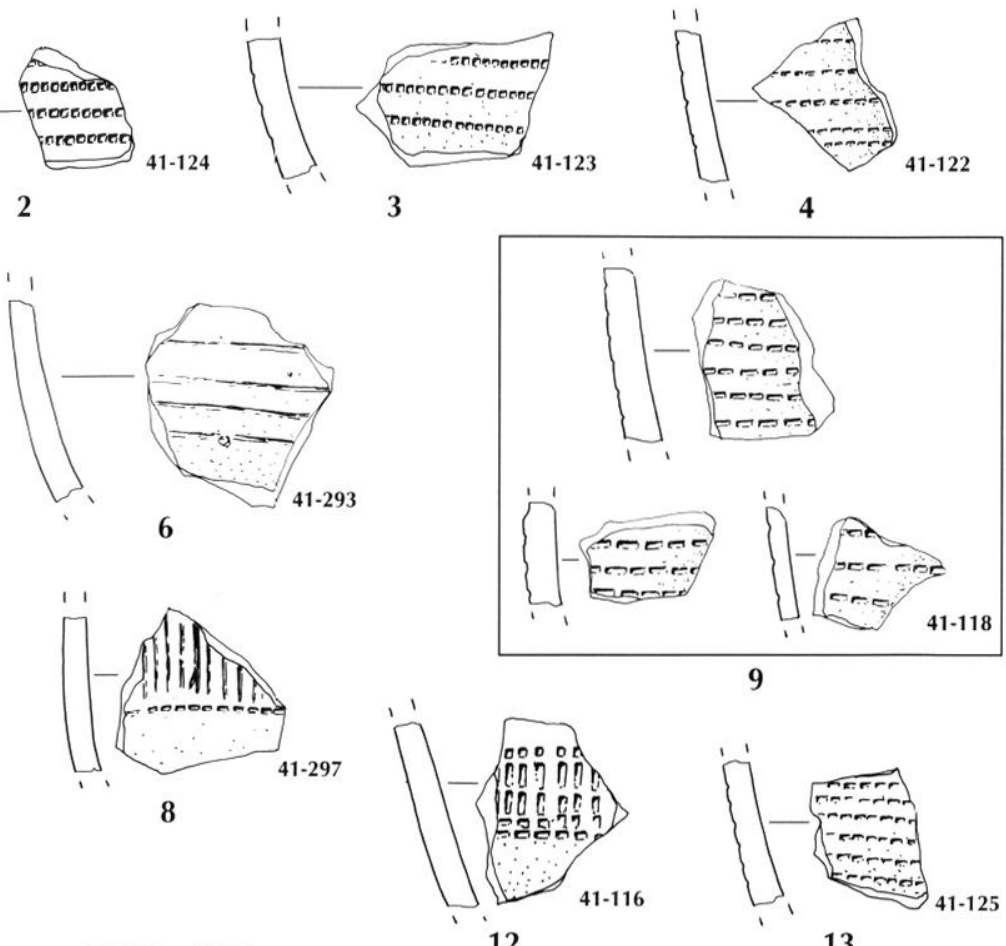

13

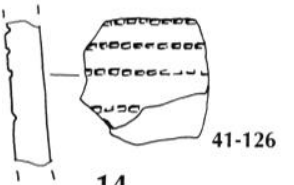

14

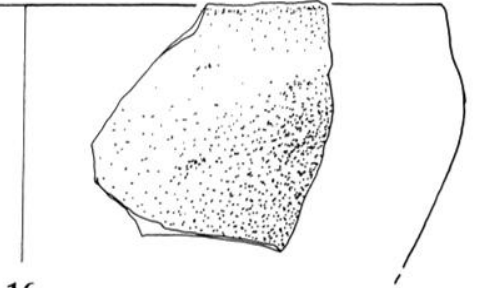

16

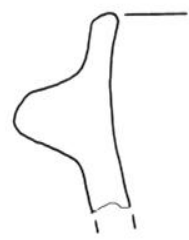

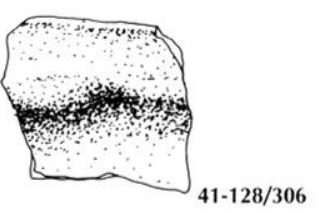

19

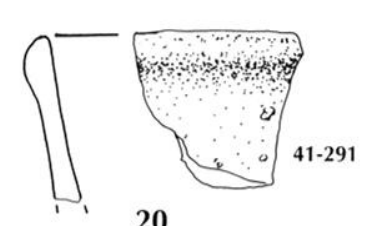

20

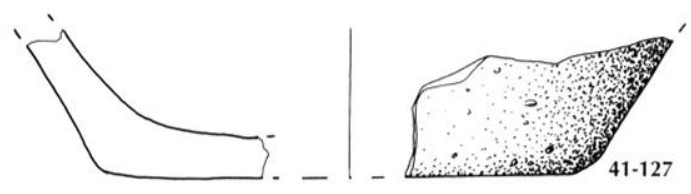

21 $5 \mathrm{~cm}$

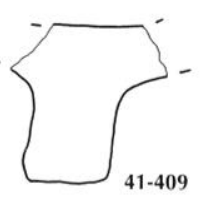

22

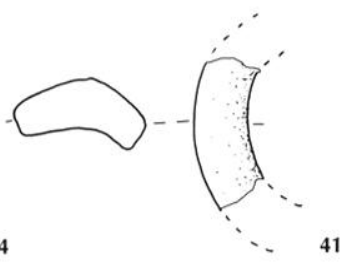

24

Fig. 30 - 1-24: céramique non décorée et décorée du locus 6, UF 41 (dessin F: Ducreux et F. Gauchet). 
UF 3 locus 1 ( 8 tessons), UF 20 locus 2 ( 4 tessons), UF 23 locus 2 ( 1 tesson), UF 24 locus 2 ( 7 tessons), UF 29 locus 2 (13 tessons), UF 39 locus 3 (9 tessons). Ces tessons n'ont pas été pris en compte dans l'étude.

\section{Fragmentation des vases}

À l'échelle du site, la fragmentation des tessons n'est pas très élevée: $13,80 \mathrm{~g}$ en moyenne par tesson ${ }^{6}$ (tabl. III).

La fragmentation est cependant différente selon les catégories de vases et selon les secteurs du site. Pour les individus vases, elle est faible $(18,59 \mathrm{~g}$ en moyenne par tesson), alors que pour les lots, elle est nettement plus importante (9,01 g en moyenne par tesson). De même, les vases décorés sont plus fragmentés du fait de la plus grande finesse de leurs parois.

À l'échelle du site, la fragmentation est particulièrement faible dans le locus 4 et surtout dans le locus 5 , alors qu'elle est élevée dans le locus 6 . Le type de structure ne semble pas influer sur cet indice. On notera cependant que dans l'UF 12 (trou de poteau du locus 5) et l'UF 1 (fosse dépotoir du locus 1), la céramique est très fragmentée, tandis que dans l'Uf 2 du locus 1 (fosse) et dans l'Uf 9 du locus 5 («céramique enterrée »), la fragmentation est moindre.

\section{Répartition de la céramique sur le site}

Si l'on considère le poids total des tessons découverts sur le site, plus de la moitié provient du locus $2(35 \%)$ et du locus 1 (23\%), c'est-à-dire des deux unités d'habitation dont la fouille a été la plus complète (tabl. II).

La céramique décorée est plus abondante dans le locus 1 (deux fois plus que dans le locus 2), essentiellement concentrée dans l'UF 2 (fosse) (fig. 31). Dans une moindre mesure, elle est également bien représentée dans la partie méridionale du locus 2. Ailleurs, la céramique décorée est plutôt rare. Le locus 5 , interprété comme une aire de stockage, ne livre même aucun tesson décoré. Toutefois, la fosse de ce locus (UF 9) a livré les restes de deux grands vases attribués à la céramique commune campaniforme. Hormis cette structure, il n'y a pas de concentration particulière de céramique commune sur le site, si l'on considère le poids total. Nous verrons que ce résultat est différent quand on prend en compte le nombre de vases (voir p. 81).

6. On considere généralement que le poids moyen d'un tesson sur un habitat pré-ou protohistorique est de $12 \mathrm{~g}$.
Tabl. III - Fragmentation des tessons relevés en stratigraphie (seuls les individus sont pris en compte).

\begin{tabular}{|c|c|c|c|c|}
\hline Zone & UF & Nombre tessons & Poids en $\mathrm{g}$ & Ind. frag.* \\
\hline $\mathrm{Z} 1$ & 1 & 14 & 139 & 9,93 \\
\hline Z1 & 2 & 30 & 588 & 19,6 \\
\hline Z1 & 3 & 13 & 251 & 19,31 \\
\hline Z1 & 15 & 9 & 143 & 15,89 \\
\hline $\mathrm{Z1}$ & 17 & 5 & 69 & 13,8 \\
\hline $\mathrm{Z1}$ & 18 & 22 & 268 & 12,18 \\
\hline Z1 & 19 & 45 & 851 & 18,91 \\
\hline $\mathrm{Z1}$ & 22 & 25 & 261,5 & 10,46 \\
\hline Z1 & 26 & 10 & 104 & 10,4 \\
\hline Total zone 1 & & 173 & 2674,5 & 15,46 \\
\hline $\mathrm{Z} 2$ & 13 & 14 & 225 & 16,07 \\
\hline $\mathrm{Z2}$ & 16 & 44 & 523,5 & 11,9 \\
\hline Z2 & 20 & 5 & 39 & 7,8 \\
\hline $\mathrm{Z} 2$ & 21 & 8 & 122 & 15,25 \\
\hline $\mathrm{Z} 2$ & 23 & 5 & 81,5 & 16,3 \\
\hline$\angle 2$ & 24 & 27 & 373 & 13,81 \\
\hline $\mathrm{Z} 2$ & 27 & 40 & 664 & 16,6 \\
\hline $\mathrm{Z} 2$ & 28 & 19 & 205 & 10,79 \\
\hline $\mathrm{Z} 2$ & 30 & 3 & 10 & 3,33 \\
\hline $\mathrm{Z2}$ & 31 & 3 & 10 & 3,33 \\
\hline Z2 & 35 & 1 & 3 & 3 \\
\hline Total zone 2 & & 169 & 2256 & 13,35 \\
\hline $\mathrm{Z3}$ & 37 & 13 & 86,5 & 6,65 \\
\hline $\mathrm{Z3}$ & 39 & 11 & 247 & 22,45 \\
\hline Total zone 3 & & 24 & 333,5 & 13,9 \\
\hline Z4 & 32 & 2 & 83,5 & 41,75 \\
\hline Z4 & 33 & 6 & 153,5 & 25,58 \\
\hline Z4 & 34 & 14 & 283 & 20,21 \\
\hline Total zone 4 & & 22 & 520 & 23,64 \\
\hline $\mathrm{Z5}$ & 9 & 62 & 2994 & 48,29 \\
\hline Z5 & 12 & 1 & 9 & 9 \\
\hline Total zone 5 & & 63 & 3003 & 47,67 \\
\hline Z6 & 41 & 47 & 472 & 10,04 \\
\hline Total zone 6 & & 47 & 472 & 10,04 \\
\hline Total site & & 498 & 9259 & 18,59 \\
\hline
\end{tabular}

* Ind. frag. = indice de fragmentation (poids/nombre tessons)

\section{Liaisons entre tessons}

Peu de collages et de regroupements ${ }^{7}$ ont pu être réalisés (tabl. IV). Dans la majorité des cas, ils correspondent à des vases décorés.

Les collages concernent 5 individus. Ils ont été effectués entre tessons issus d'une même UF: dans trois cas du locus 2, dans un cas du locus 1 et dans un cas du locus 6.

Les regroupements, quant à eux, concernent 10 individus. Dans quatre cas, il s'agit de tessons issus d'une

7. Nous distinguons les regroupements des collages. I e's regroupements concernent au moins 2 tessons qui présentent les mêmes caractéristiques typologiques et techniques et qui, de ce fait, doirent appartenir au même récipient, sans quaucun collage puisse être effectué. 


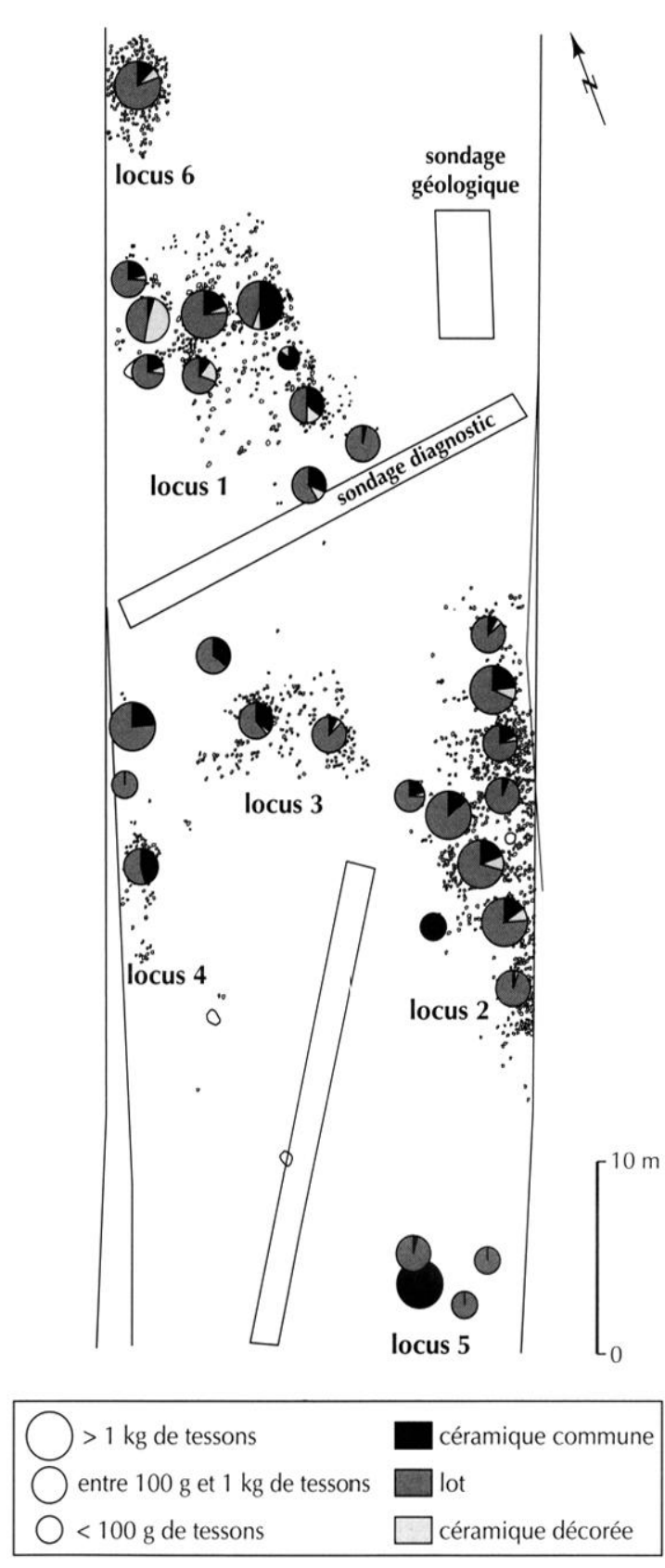

Fig. 31 - Répartition de la céramique sur le gisement de La Noue.

même structure, dans les locus 1, 3, 4 et 6 . Dans les six cas restant, trois regroupements ont été réalisés entre tessons découverts dans plusieurs UF du locus 2, un entre une fosse du locus 1 et une UF du locus 2, un entre une UF du locus 1 et une UF du locus 4 et le dernier entre deux UF du locus 4 (fig. 32).

D'une manière générale, les liaisons entre tessons sont plus fréquentes dans le locus 2. Les rares regroupements inter-zones unissent le locus 1 et locus 2 ainsi que le locus 1

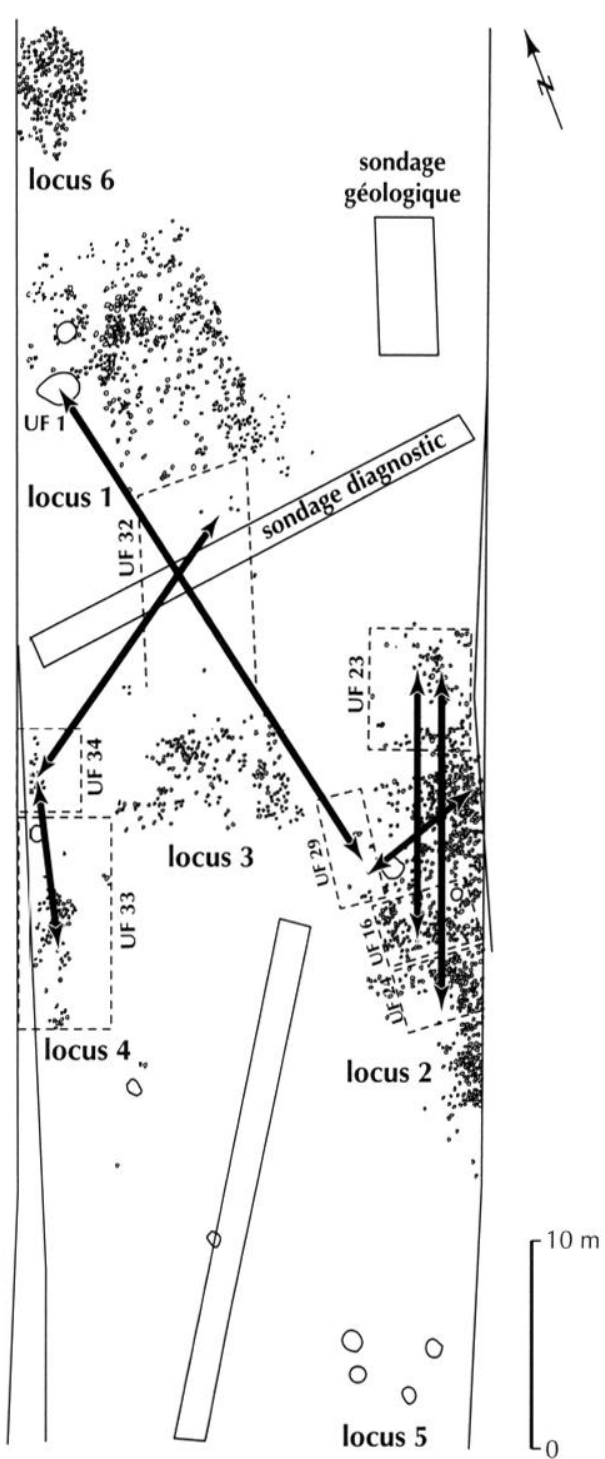

Fig. 32 - Plan général des liaisons entre tessons.

et le locus 4, assurant ainsi la contemporanéité de fonctionnement de ces trois zones.

L. S.

\section{CARACTÉristiques TeChNiQues DES VASES}

\section{Les surfaces}

Dans la quasi-totalité des cas, les surfaces des tessons sont érodées, si bien que le dégraissant est visible en surface et que les traitements de surface (lissage, polissage) ont disparu.

Certains tessons sont brûlés (tabl. V). Ils sont particulièrement nombreux dans les locus 2 et 4 et, dans une moindre mesure, dans la partie méridionale du locus 1 . Lors de la fouille, aucune trace d'incendie ou de structures de com- 
Tabl. IV - Liaisons entre tessons.

\begin{tabular}{|c|c|c|}
\hline \multicolumn{3}{|l|}{ Regroupements } \\
\hline Ref. tesson & Localisation de la structure & $\mathrm{Nb}$ tessons \\
\hline UF $1-318+$ UF $29-99$ & fosse du locus $1+$ UF du locus 2 & 3 \\
\hline UF $18-279+180$ & locus 1 & 2 \\
\hline UF $23-56$ + UF $24-247$ & locus 2 & 2 \\
\hline UF $16-73+$ UF $23-55$ & locus 2 & 2 \\
\hline UF $27-14+$ UF $29-38$ & niveau et UF du locus 2 & 2 \\
\hline UF $39-191+193$ & locus 3 & 2 \\
\hline UF $34-146+147$ & locus 4 & 2 \\
\hline UF $33-139+$ UF $34-148$ & locus 4 & 2 \\
\hline UF $41-118$ & locus 6 & 3 \\
\hline UF $32-173+$ UF $34-152$ & UF au sud du locus $1+$ UF du locus 4 & 2 \\
\hline \multicolumn{3}{|l|}{ Collages } \\
\hline Ref. tesson & Localisation de la structure & $\mathrm{Nb}$ tessons \\
\hline UF $19-265+266+267+264$ & locus 1 & 6 \\
\hline UF $21-243+244$ & locus 2 & 2 \\
\hline UF $16-71+76$ & locus 2 & 2 \\
\hline UF $16-78+200$ & locus 2 & 2 \\
\hline UF $41-128+306$ & locus 6 & 2 \\
\hline
\end{tabular}

Tabl. V - Répartition des tessons brûlés. Faune brûlée: voir l'étude de A. Argant, p. 50-51.

\begin{tabular}{|c|c|c|c|}
\hline Zone & UF & Tessons brûlés & Faune brûlée \\
\hline$Z 1$ & 3 & $X$ & \\
\hline$Z 1$ & 19 & $X$ & \\
\hline$Z 1$ & 22 & $X$ & \\
\hline$Z 1$ & 26 & $X$ & \\
\hline$Z 2$ & 13 & $X$ & $X$ \\
\hline$Z 2$ & 16 & $X$ & \\
\hline$Z 2$ & 20 & $X$ & \\
\hline$Z 2$ & 21 & $X$ & \\
\hline$Z 2$ & 23 & $X$ & \\
\hline$Z 2$ & 24 & $X$ & \\
\hline$Z 2$ & 27 & $X$ & \\
\hline$Z 2$ & 29 & $X$ & \\
\hline$Z 2$ & 30 & $X$ & \\
\hline$Z 2$ & 31 & $X$ & \\
\hline$Z 4$ & 32 & $X$ & \\
\hline$Z 4$ & 33 & $X$ & \\
\hline$Z 4$ & 34 & $X$ & \\
\hline$Z 5$ & 12 & & \\
\hline$Z 6$ & 41 & $X$ & \\
\hline
\end{tabular}

bustion n'a été repérée. Toutefois, l'étude de la faune a également mis en évidence des traces de combustion sur les os, de façon récurrente dans le locus 2 et dans l'UF 12 de la zone 5.

En ce qui concerne les couleurs, plus des trois quarts des surfaces externes sont orange (tabl. VI). Excepté le beige,
Tabl. VI - Couleur des surfaces externes et atmosphères de cuisson (en nombre de vases).

\begin{tabular}{|c|c|c|c|c|c|}
\hline \multirow[b]{2}{*}{$\begin{array}{r}\text { Couleur } \\
\text { extérieure }\end{array}$} & \multicolumn{4}{|c|}{ Atmosphères de cuisson } & \multirow[b]{2}{*}{ Total } \\
\hline & oxydante & $\begin{array}{c}\text { partiellement } \\
\text { oxydante }\end{array}$ & réductrice & $\begin{array}{c}\text { partiellement } \\
\text { réductrice }\end{array}$ & \\
\hline bordeaux & & 1 & & & 1 \\
\hline gris & & & 2 & 3 & 5 \\
\hline noir & & & 7 & 2 & 9 \\
\hline marron & & & 18 & 5 & 23 \\
\hline beige & 10 & 38 & & & 48 \\
\hline orange & 72 & 213 & & & 285 \\
\hline Total & 82 & 252 & 27 & 10 & 371 \\
\hline
\end{tabular}

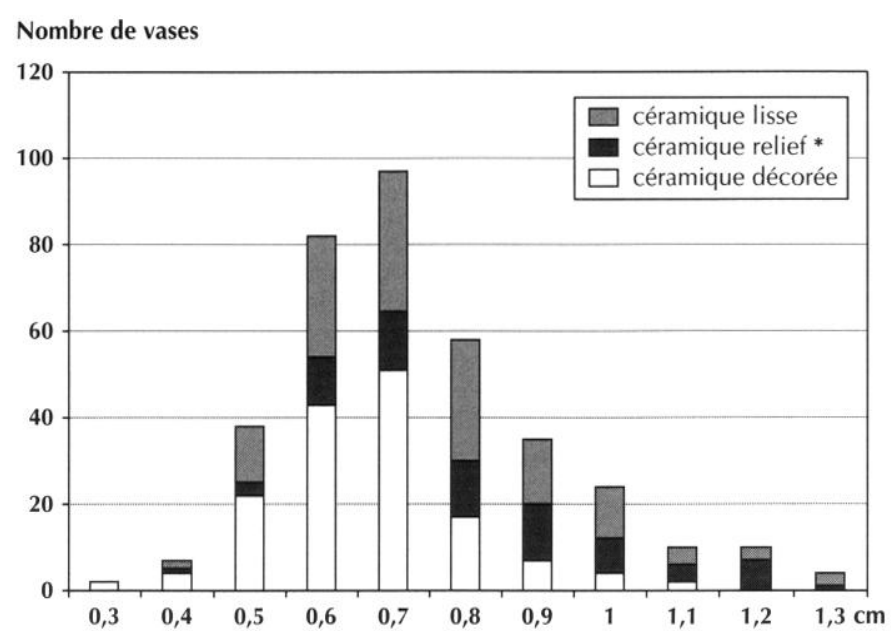

* céramique à éléments modelés (cordon, anse, mamelon, bouton, cannelure) hormis les pieds et 5 anses décollées (l'épaisseur du vase n'est pas connue)

Fig. 33 - Distribution des épaisseurs de parois par catégorie de vases.

bien représenté (13\%), les autres couleurs sont anecdotiques (bordeaux, gris, noir, marron) et elles sont très rarement associées aux vases décorés. La couleur des surfaces internes et du cœur des tessons montre que la quasi-totalité des vases ont été cuits en atmosphère oxydante $(68 \%)$ ou partiellement oxydante $(22 \%)$.

\section{Montage et épaisseur des parois}

Les décollements de colombin sont assez rares. Ils présentent des profils en tuile ou en biseau.

Certaines formes sont également complétées par modelage (voir p. 78).

La céramique du site de La Noue est par ailleurs relativement épaisse : la moitié a une épaisseur comprise entre 0,7 et $0,9 \mathrm{~cm}$ (fig. 33). Elle est très rarement inférieure à $0,6 \mathrm{~cm}$.

Les épaisseurs ne sont pas vraiment corrélées aux formes des vases. On notera toutefois que les formes basses sont 
plus épaisses. En revanche, les vases décorés sont plus fins, même si la plupart d'entre eux ont une épaisseur de $0,7 \mathrm{~cm}$. Les vases lisses et les vases munis de préhensions ou de cordons figurent dans les catégories les plus épaisses. Ceci est sans doute imputable à leur taille, généralement supérieure à celle des autres types de récipients, et à leur fonction de stockage ou de transport.

L. S.

\section{TYPOLOGIE DES FORMES}

Les éléments morphologiques sont peu fréquents: la forme est identifiable pour 55 vases. Pour un tiers des individus, la forme est indéterminée.

La fragmentation nous oblige à étudier les différentes parties du vase séparément, mais une typologie sommaire peut être proposée en vue d'intégrer la série dans un contexte chronoculturel plus large.

\section{Bord et diamètre de l'ouverture}

Pour $11 \%$ des individus, le col du vase est connu. Il est possible de distinguer six types, parmi lesquels les cols convergents et les cols divergents apparaissent dans des proportions à peu près égales (fig. 34).
Les diamètres à l'ouverture sont compris entre 11 et $35,5 \mathrm{~cm}$, mais ils mesurent le plus souvent entre 11 et $20 \mathrm{~cm}$.

Étant donné les effectifs, aucune corrélation entre le type du col et le diamètre à l'ouverture n'est perceptible.

\section{Fond et diamètre de l'assise}

Pour $10 \%$ des individus, le fond du vase est connu. Nous avons distingué quatre types, parmi lesquels le fond plat simple domine (fig. 35).

Les diamètres des assises sont compris entre 5 et $19,5 \mathrm{~cm}$, mais ils sont le plus souvent inférieurs à $10 \mathrm{~cm}$.

Il ne semble pas y avoir de corrélation entre la taille de l'assise et la forme du vase: ainsi, le grand récipient découvert enterré dans le locus 5 possède un fond relativement étroit, de $8,5 \mathrm{~cm}$ (fig. $29, \mathrm{n}^{\circ} 2$ ). Aucune corrélation n'est également perceptible entre le type de fond et le diamètre de l'assise.

\section{Distribution typologique}

Pour classer les formes reconstituables de la série de La Noue, nous nous sommes appuyée sur la typologie élaborée par B. Othenin-Girard à partir du corpus de Noir Bois

\begin{tabular}{|c|c|c|c|c|c|c|c|}
\hline & $\mid$ & \begin{tabular}{|l|l|}
$\mid$ & $\mid$ \\
bord droit \\
convergent
\end{tabular} & $\begin{array}{l}(|+| \\
\text { bord convexe } \\
\text { convergent }\end{array}$ & \begin{tabular}{l|}
$\mid$ \\
bord droit \\
divergent
\end{tabular} & \begin{tabular}{l|}
$\mid$ \\
bord concave \\
divergent
\end{tabular} & $\begin{array}{c}\mid \\
\text { bord sinueux } \\
\text { convergent }\end{array}$ & TOTAL \\
\hline $\mathrm{Nb}$ vases & 2 & 4 & 9 & 13 & 9 & 4 & 41 \\
\hline 11 à $16 \mathrm{~cm}$ & & & 3 & 7 & 4 & & 14 \\
\hline 16 à $20 \mathrm{~cm}$ & 2 & 3 & 1 & 3 & 3 & 1 & 13 \\
\hline 20 à $25 \mathrm{~cm}$ & & & 3 & 1 & 1 & 1 & 6 \\
\hline$\geq 25 \mathrm{~cm}$ & & 1 & 2 & 2 & 1 & 2 & 8 \\
\hline
\end{tabular}

Fig. 34 - Typologie des bords et diamètres de l'ouverture.

\begin{tabular}{|c|c|c|c|c|c|}
\hline & fond plat & fond ombiliqué & fond plat démarqué & fond plat débordant & TOTAL \\
\hline $\mathrm{Nb}$ vases & 19 & 1 & 10 & 6 & 36 \\
\hline 5 à $8 \mathrm{~cm}$ & 3 & & 2 & 3 & 8 \\
\hline 8 à $10 \mathrm{~cm}$ & 7 & 1 & 4 & 2 & 14 \\
\hline$\geq 10 \mathrm{~cm}$ & 9 & & 4 & 1 & 14 \\
\hline
\end{tabular}

Fig. 35 - Typologie des fonds et diamètres des assises. 
(Othenin-Girard dir., 1997, fig. 41). Cette typologie prend en compte le profil et le diamètre de l'ouverture des vases, les deux critères les mieux connus dans la série de La Noue. Nous avons ainsi distingué sept types morphologiques certains et quatre probables (fig. 36).

Les jarres: avec 24 exemplaires, c'est la catégorie la mieux connue. Les jarres se distinguent des gobelets par un diamètre à l'ouverture plus grand $(>16 \mathrm{~cm})$ et également par un type de bord (convergent sinueux). Elles portent le plus souvent un cordon préoral (14 exemplaires).

Les gobelets: cette catégorie est également bien représentée (17 exemplaires). Le profil est sinueux et les bords divergents, droits ou concaves. La plupart portent un décor (11 exemplaires).

La faisselle: ce type n'existe pas à Noir Bois. D'un point de vue morphologique, il aurait pu être classé dans la catégorie des écuelles. Sa surface est entièrement perforée.

Les bols hauts: cette forme n'existe pas non plus à Noir Bois. Il n'y a que 2 exemplaires identifiables à La Noue. Ils ont un profil courbe et convexe et des bords convergents. Ils sont à la limite de la définition de la forme haute (diamètre < hauteur) et de celle de la forme basse (diamètre $>$ hauteur), autant que l'on puisse en juger sur les reconstitutions graphiques de ces deux vases. Ils ne portent pas de décor, mais l'un d'eux présente une cannelure sous le bord.

Les bols: le profil est courbe et convexe, les bords convergents. Dans un seul cas, le diamètre à l'ouverture est petit $(<16 \mathrm{~cm})$ et 3 exemplaires sur 7 portent un cordon préoral.

L'écuelle: ce type n'est représenté que par un seul exemplaire, à profil évasé. Son diamètre à l'ouverture est petit et il ne porte pas de décor.

La jatte: les trois exemplaires de jatte ne portent aucun décor. La rupture de pente entre le col et la panse est marquée par une cannelure ou une fausse carène.

Enfin, nous supposons l'existence de quatre types supplémentaires, représentés seulement par des fragments de vascs dont lc profil n'est pas reconstituable.

Vase à mamelon sous le bord: un tesson de bord découvert dans le locus 6 porte un mamelon, mais sa forme est indéterminée.

Coupes polypodes: 2 exemplaires de pieds ont été découverts sur le site de La Noue, l'un hors stratigraphie et l'autre dans le locus 6 . Ils ne semblent pas appartenir au même individu. Ces éléments sont caractéristiques des coupes polypodes.

Pichets ou cruches: 8 anses en boudin, dont 2 à tenons, proviennent de différents secteurs du site. Elles doivent vraisemblablement appartenir à des cruches ou pichets. Cependant, l'une d'elles, qui devait être fixée horizontalement sur le vase, pourrait appartenir à une forme basse et large, comme on en connaît en Europe centrale et dans le. nord de l'Italie.

L. S.

\section{MODIFICATION DES SURFACES: DÉCORS EN CREUX ET ÉLÉMENTS EN RELIEF}

Les modifications de surface, réalisées avant cuisson sur 234 vases, sont de deux ordres: les décors en creux et les éléments en relief (préhensions, cordons, pieds...).

Les décors imprimés représentent plus de la moitié des modifications de surface $(59 \%)$ et le modelage un tiers (tabl. VII). Signalons enfin un cas de perforation (perforations circulaires dans la pâte molle de $0,5 \mathrm{~cm}$ de diamètre), correspondant à l'unique exemplaire de faisselle découverte sur le site.

Les techniques sont très rarement associées sur un même individu: deux vases seulement portent à la fois un élément modelé et des impressions, et deux autres un élément modelé et des incisions (fig. 21, $n^{\circ} 17$; fig. 25, $n^{\circ} 17$; fig. $26, n^{\circ} 13$ et fig. $28, n^{\circ} 14$ ).

\section{Décors imprimés et incisés}

Parmi les décors en creux (tabl. VIII), l'impression prédomine largement (94\% des décors en creux), essentiellement réalisée au peigne (91\% des décors imprimés). L'impression d'ongle est attestée, mais en faible quantité, de même que l'impression de poinçon (ovale le plus souvent, un seul cas de poinçon quadrangulaire) et l'impression de spatule. Sur un vase seulement, deux outils ont été employés pour le décor: un peigne et un poinçon ovale (fig. 22, n'2).

Étant donné l'érosion des surfaces et la fragmentation des vases, il est difficile de caractériser précisément les

Tabl. VII - Techniques de modification des surfaces.

\begin{tabular}{|l|c|}
\hline Techniques & Nombre de vases \\
\hline impression & 139 \\
\hline incision & 8 \\
\hline décors en creux indéterminés & 5 \\
\hline modelage + impression & 2 \\
\hline modelage + incision & 2 \\
\hline modelage & 77 \\
\hline perforation & 1 \\
\hline Total & $\mathbf{2 3 4}$ \\
\hline
\end{tabular}




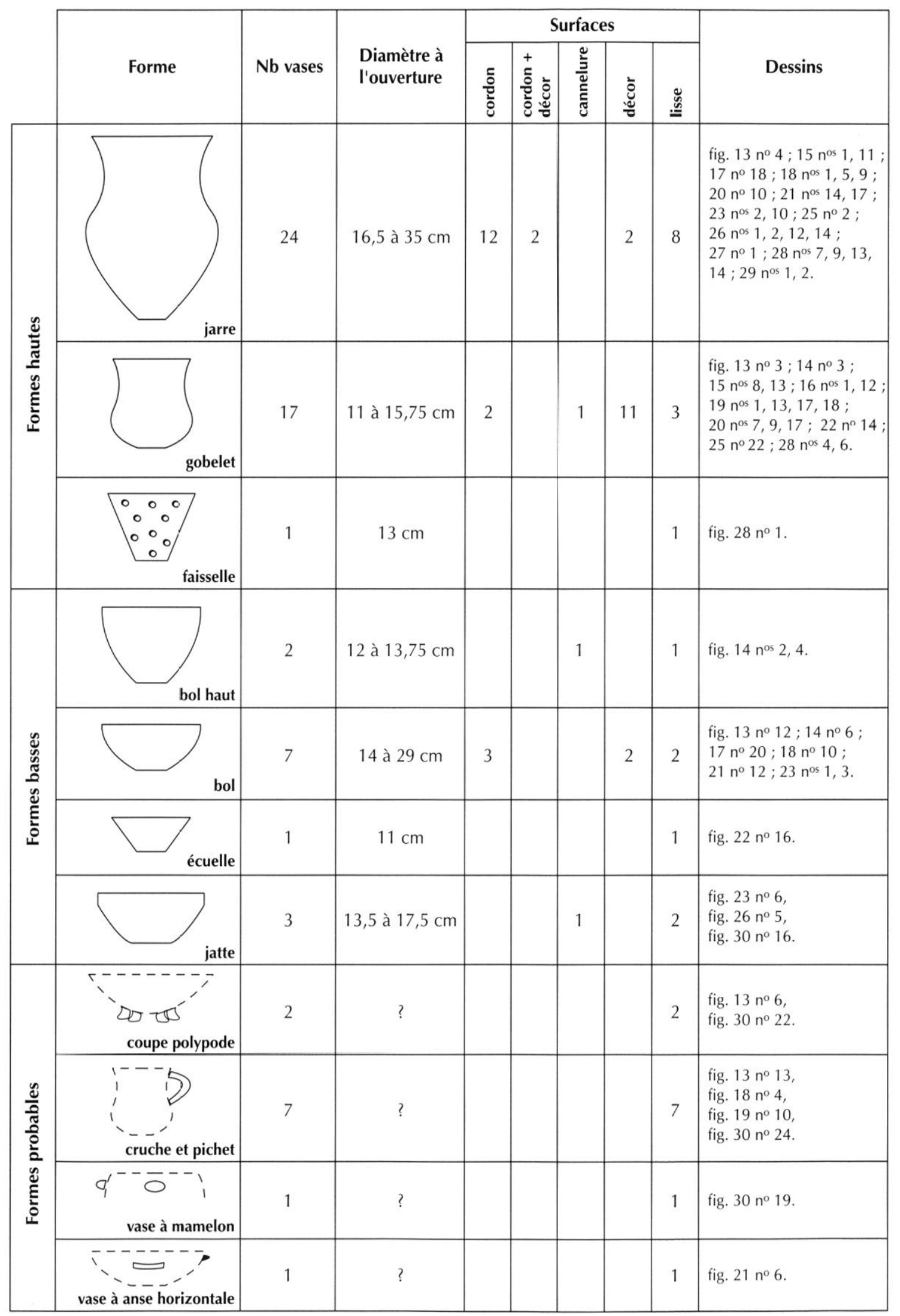

Fig. 36 - Typologie des vases de La Noue. 
Tabl. VIII - Outils employés pour les décors en creux.

\begin{tabular}{|l|c|c|c|c|c|c|}
\cline { 2 - 7 } \multicolumn{1}{c|}{} & \multicolumn{5}{c|}{ Outils } & \multicolumn{1}{c|}{} \\
\hline Techniques & peigne & ongle & spatule & poinçon & indéterminé & Total \\
\hline impression & 126 & 10 & 1 & 4 & 1 & $\mathbf{1 4 2}$ \\
\hline incision & & & & 10 & & $\mathbf{1 0}$ \\
\hline indéterminés & & & & & 5 & $\mathbf{5}$ \\
\hline Total & $\mathbf{1 2 6}$ & $\mathbf{1 0}$ & $\mathbf{1}$ & $\mathbf{1 4}$ & $\mathbf{6}$ & $\mathbf{1 5 7}$ \\
\hline
\end{tabular}

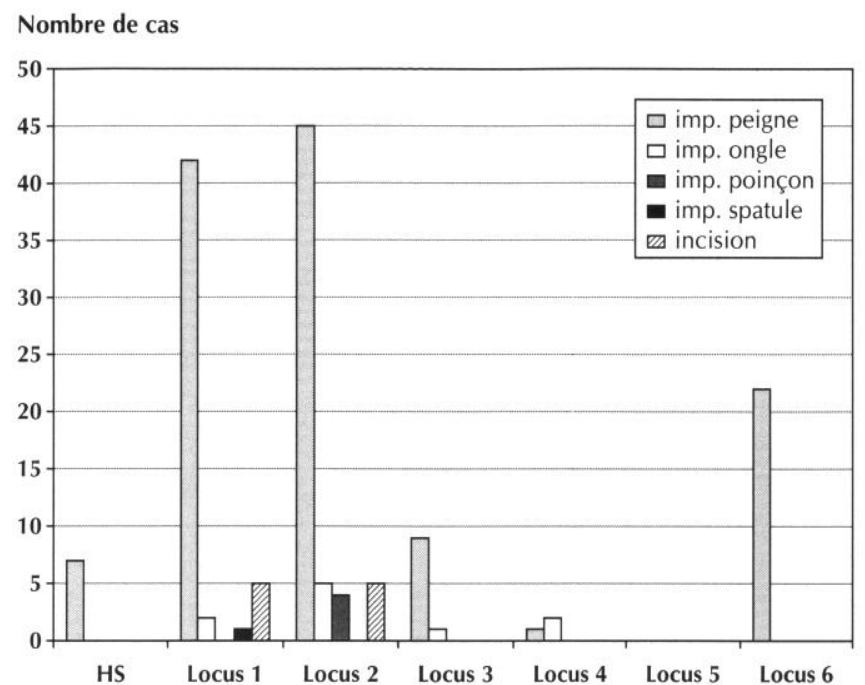

Fig. 37 - Répartition des techniques décoratives par locus.

peignes utilisés pour la réalisation des décors. Hormis un exemplaire à dents ovales, ils sont munis de dents quadrangulaires. En outre, ils ne se distinguent pas par leur finesse puisque la grande majorité d'entre eux ont des dents d'au moins $2 \mathrm{~mm}$ de long (tabl. IX).

En ce qui concerne la répartition des décors en creux, ils sont plus abondants dans le locus 2 et dans le locus 1 , qui totalisent à eux deux les trois quarts des décors (respectivement $40 \%$ et $33 \%$; fig. 37). Ils sont très faiblement représentés dans le locus 4 ( 3 vases) et ils sont absents du locus 5 . Dans le locus 2, les outils sont également plus diversifiés. Bien que les proportions fluctuent, l'impression de peigne et l'impression d'ongle sont présentes dans presque tous les locus; au contraire, l'impression de poinçon n'est attestée que dans le locus 2 et l'impression de spatule ne l'est que dans le locus 1. L'incision n'apparaît que dans les locus 1 et 2 , dans des proportions identiques.

Pour ce qui est des thèmes décoratifs, parmi les 156 vases décorés, les lignes horizontales prédominent largement ( $70 \%$ des décors, dont $15 \%$ en thème unique; tabl. X). Le répertoire ornemental est par ailleurs assez restreint:
Tabl. IX - Taille des dents des peignes.

\begin{tabular}{|l|c|}
\hline \multicolumn{1}{|c|}{ Taille dent } & Nombre de vases \\
\hline $1,5 \times 1 \mathrm{~mm}$ & 3 \\
\hline $1,5 \times 1,5 \mathrm{~mm}$ & 16 \\
\hline $2 \times 1 \mathrm{~mm}$ & 2 \\
\hline $2 \times 1,5 \mathrm{~mm}$ & 14 \\
\hline $2,5 \times 1,5 \mathrm{~mm}$ & 6 \\
\hline $2 \times 2 \mathrm{~mm}$ & 1 \\
\hline$>2 \mathrm{~mm}$ & 4 \\
\hline indéterminé & 80 \\
\hline Total & $\mathbf{1 2 6}$ \\
\hline
\end{tabular}

Tabl. X - Motifs et thèmes décoratifs.

\begin{tabular}{|l|c|}
\hline \multicolumn{1}{|c|}{ Motifs } & Nombre de cas \\
\hline ligne horizontale & 113 \\
\hline échelle & 11 \\
\hline bande hachurée & 8 \\
\hline chevron & 7 \\
\hline croisillon & 5 \\
\hline triangle & 5 \\
\hline métope & 1 \\
\hline fermeture éclair & 1 \\
\hline inorganisé & 1 \\
\hline indéterminé & 12 \\
\hline Total récurrences & $\mathbf{1 6 4}$ \\
\hline
\end{tabular}

échelles, bandes hachurées (souvent contractées ou en sens unique), chevrons (généralement emboîtés), bandes de croisillons, triangles rayés alignés, métope et motif en "fermeture Éclair".

Les lignes ont été réalisées à l'aide de techniques variées, néanmoins $91 \%$ ont été tracés par impression de peigne (fig. 38). Les motifs en métope et en "fermeture Éclair » ainsi que les bandes de croisillons n'ont été traités que par impression, alors que les chevrons, les triangles et les échelles ont été réalisés le plus souvent par impression et, dans une moindre mesure, par incision.

Il est difficile de croiser les données issues de l'étude des décors avec celles obtenues sur les formes: pour $79 \%$ des vases décorés, la forme est indéterminée. Toutefois, parmi les 17 formes reconstituables décorées, les gobelets sont majoritaires, au contraire des formes basses, représentées seulement par deux bols (fig. 36).

En ce qui concerne la répartition des décors sur le site, le répertoire ornemental du locus 1 est à peu près identique à celui du locus 2 , cependant les vases décorés du locus 2 portent des motifs plus variés (tabl. XI). Les motifs en croi- 


\begin{tabular}{|c|c|c|c|c|c|c|c|c|c|c|c|}
\hline \multicolumn{2}{|c|}{ Techniques } & \multirow{2}{*}{$\begin{array}{c}\text { Lignes } \\
\\
103\end{array}$} & \multirow{2}{*}{$\begin{array}{c}\text { Métope } \\
\text { mz曲 } \\
\text { पm曲 } \\
1\end{array}$} & \multirow[t]{2}{*}{$\begin{array}{c}\text { Inorganisé } \\
\text { ',',',', }\end{array}$} & \multirow{2}{*}{$\begin{array}{c}\text { F. Éclair } \\
1\end{array}$} & \multirow{2}{*}{ 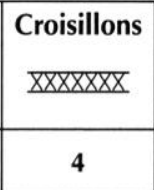 } & \multirow{2}{*}{ Chevrons } & \multirow{2}{*}{$\begin{array}{c}{ }_{4}^{\text {Triangles }} \\
\nabla\end{array}$} & \multirow{2}{*}{$\begin{array}{c}\text { Hachures } \\
\text { पापापा } \\
6\end{array}$} & \multirow{2}{*}{$\begin{array}{c}\text { Échelles } \\
\text { माप्माप } \\
8\end{array}$} & \multirow{2}{*}{$\begin{array}{c}\text { Indéterminé } \\
? \\
2\end{array}$} \\
\hline \multirow{4}{*}{ 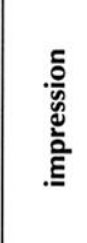 } & peigne & & & & & & & & & & \\
\hline & ongle & 2 & & 1 & & & & & & & 7 \\
\hline & poinçon & 2 & & & 1 & & & & & & 1 \\
\hline & spatule & 1 & & & & & & & & & \\
\hline \multicolumn{2}{|r|}{ incision } & 2 & & & & & 2 & 1 & 2 & 1 & 2 \\
\hline \multicolumn{2}{|c|}{ indéterminé } & 3 & & & & 1 & & & & 2 & \\
\hline
\end{tabular}

Fig. 38 - Corrélation des thèmes et des techniques décoratives.

Tabl. XI - Répartition des motifs et des thèmes par locus.

\begin{tabular}{|l|c|c|c|c|c|c|c|c|c|c|}
\hline & Ligne horiz. & Hachure & Échelle & Chevron & Triangle & Croisillon & Métope & Inorg. & F. Éclair & Indét. \\
\hline HS & 6 & 1 & 1 & & & & & & & \\
\hline Locus 1 & 43 & 2 & 1 & 2 & 2 & 4 & & & & 3 \\
\hline Locus 2 & 37 & 5 & 6 & 3 & 2 & 1 & 1 & & 1 & 8 \\
\hline Locus 3 & 8 & & & 1 & & & & 1 & & \\
\hline Locus 4 & 2 & & & & & & & & & 1 \\
\hline Locus 5 & & & & & & & & & & \\
\hline Locus 6 & 19 & & 3 & 1 & 1 & & & & & \\
\hline
\end{tabular}

sillon sont au contraire concentrés dans le locus 1. Dans les locus 4 et 6 , les décors sont plus monotones, et dans le locus 5 , ils sont absents.

Quant à l'agencement des décors sur le vase, les informations sont rares, vu la fragmentation de la série. Autant que l'on puisse en juger, le décor est couvrant et il s'organise selon deux modèles: panneau de lignes horizontales du bord au fond du vase ou registres larges composés de plusieurs bandes horizontales accolées. Un seul exemplaire, le plus complet de la série décorée, présente une organisation un peu différente, avec l'intégration d'un motif en métope au sein du registre central (fig. 22, $n^{\circ} 14$ ).

La taille des tessons ne permet pas une étude précise de la symétrie du décor. La translation des thèmes semble cependant prédominer.

\section{Éléments modelés}

Les cordons représentent les trois quarts des éléments modelés. Trois types se distinguent: le cordon à section en " $U$ ", le cordon à section en " $V$ " et le cordon plat. Ils ont été modelés puis collés sur la surface du vase ou directement modelés dans la masse.

Dans la série de La Noue, trois quarts des cordons ont une section en " $U$ " (tabl. XII). Ils sont généralement
Tabl. XII - Morphologie et technique de façonnage des cordons.

\begin{tabular}{|l|c|c|c|}
\hline \multirow{2}{*}{\multicolumn{1}{|c|}{ Cordon }} & \multicolumn{2}{c|}{ Technique } & \multirow{2}{*}{ Total } \\
\cline { 2 - 3 } & collé & dans la masse & \\
\hline Cordon en U & 13 & 33 & $\mathbf{4 6}$ \\
\hline Cordon en V & 4 & 4 & $\mathbf{8}$ \\
\hline Cordon plat & 1 & & $\mathbf{1}$ \\
\hline Cordon indéterminé & 6 & & $\mathbf{6}$ \\
\hline Total & $\mathbf{2 4}$ & $\mathbf{3 7}$ & $\mathbf{6 1}$ \\
\hline
\end{tabular}

modelés dans la masse ( $72 \%$ des cas). Quel que soit le type des cordons, ils sont le plus souvent fins: pour la moitié d'entre eux, la largeur est comprise entre 0,6 et $0,8 \mathrm{~cm}$, avec une nette prédominance de $0,6 \mathrm{~cm}$ (tabl. XIII). Hormis l'unique exemple de cordon plat (fig. 21, no 11), collé directement sur la lèvre, les cordons sont placés sous le bord, à une distance comprise entre 1 et $2 \mathrm{~cm}$ de l'ouverture des vases, très rarement moins et rarement plus (tabl. XIV). On note donc une certaine standardisation dans la position des cordons, sans doute en rapport avec leur fonction. En ce qui concerne leur répartition sur le site, ils proviennent pour la plupart des locus 1 et 2 (fig. 39). Le locus 1 montre une plus grande diversité dans les types; cependant, comme dans le locus 2, le type en " $U$ " modelé dans la masse prédomine nettement. L'unique exemple de cordon plat est issu du 


\begin{tabular}{|c|c|c|c|c|c|c|}
\hline \multirow{2}{*}{ Cordon } & \multicolumn{5}{|c|}{ Largeur } & \multirow{2}{*}{ Total } \\
\hline & $<0,6 \mathrm{~cm}$ & 0,6 à $0,8 \mathrm{~cm}$ & 0,8 à $1 \mathrm{~cm}$ & $>1 \mathrm{~cm}$ & indéterminé & \\
\hline Cordon en $U$ & 13 & 23 & 8 & 2 & & 46 \\
\hline Cordon en V & & 5 & 2 & 1 & & 8 \\
\hline Cordon plat & & & & 1 & & 1 \\
\hline Cordon indéterminé & & 2 & & & 4 & 6 \\
\hline Total & 13 & 30 & 10 & 4 & 4 & 61 \\
\hline
\end{tabular}

Tabl. XIII - Largeur des cordons.

\begin{tabular}{|l|c|c|c|c|c|c|}
\hline \multirow{2}{*}{\multicolumn{1}{|c|}{ Cordon }} & \multicolumn{5}{|c|}{ Distance par rapport au bord du vase } & \multirow{2}{*}{ Total } \\
\cline { 2 - 6 } & $<\mathbf{1} \mathbf{~ c m}$ & $\mathbf{1} \mathbf{a} \mathbf{1 , 5} \mathbf{~ c m}$ & $\mathbf{1 , 6} \mathbf{a} \mathbf{2} \mathbf{~ c m}$ & $>\mathbf{2} \mathbf{~ c m}$ & indéterminé & \\
\hline Cordon en U & 1 & 12 & 19 & 5 & 9 & $\mathbf{4 6}$ \\
\hline Cordon en $\mathrm{V}$ & & 5 & 1 & 1 & 1 & $\mathbf{8}$ \\
\hline Cordon plat & 1 & & & & & $\mathbf{1}$ \\
\hline Cordon indéterminé & & 1 & 1 & 2 & 2 & $\mathbf{6}$ \\
\hline Total & 2 & 18 & 21 & 8 & 12 & $\mathbf{6 1}$ \\
\hline
\end{tabular}

Tabl. XIV - Distance des cordons par rapport au bord des vases.

Nombre de vases

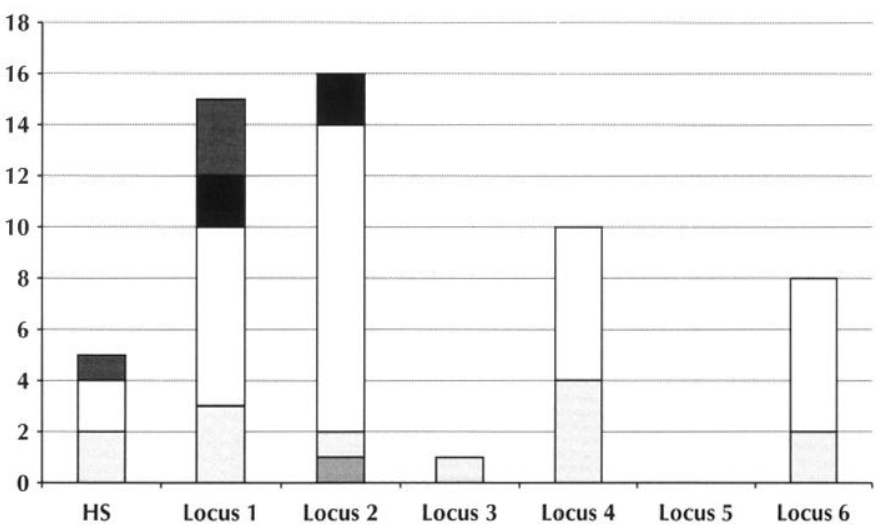

cordon en $\mathrm{V}$ modelé dans la masse cordon en $U$ modelé dans la masse

cordon en $\mathrm{V}$ collé cordon en $\mathrm{U}$ collé

Fig. 39 - Répartition des types de cordons par locus.

locus 2. Un seul cordon provient du locus 3, alors que le locus 5 n'en a livré aucun exemplaire.

Les autres catégories d'éléments modelés sont bien plus rares sur le site (tabl. XV). Parmi celles-ci, les anses en boudin sont les mieux représentées. Deux d'entre elles, issues du locus 1, ont des tenons à chaque extrémité (fig. 19, $\mathrm{n}^{\circ} 10$ ). Cinq vases, majoritairement du locus 1 , présentent des cannelures sous le bord. Des éléments de pieds, modelés et collés sur l'assise de coupes polypodes, ont été identifiés dans le locus 6 et dans un ensemble hors stratigraphie. Enfin, un vase, aussi découvert hors stratigraphie, porte un bouton collé sur la lèvre et un autre, issu du locus 6 , un
Tabl. XV - Autres éléments modelés.

\begin{tabular}{|l|c|c|c|c|}
\hline & Nombre & Technique & Largeur & Remarque \\
\hline Pied & 2 & collé & 1,9 à $2 \mathrm{~cm}$ & \\
\hline Anse en boudin & 8 & collé & 1,7 à $5,3 \mathrm{~cm}$ & 2 à tenons \\
\hline Bouton & 1 & collé & $1,5 \mathrm{~cm}$ & sur la lèvre \\
\hline Mamelon & 1 & collé & $4,6 \mathrm{~cm}$ & \\
\hline Fausse carène & 1 & modelé & & \\
\hline Cannelure & 5 & modelé & 0,5 à $0,7 \mathrm{~cm}$ & sous le bord \\
\hline Arrachement & 2 & collé & $?$ & \\
\hline Total & $\mathbf{2 0}$ & \multicolumn{5}{|l}{} \\
\hline
\end{tabular}

mamelon collé sur le col. Un cas de "fausse carène ", c'està-dire une rupture de pente provoquée par épaississement des parois et par modelage dans la masse, complète cet inventaire. Globalement, les anses constituent donc le type majoritaire des éléments modelés hors cordon. La série issue du locus 6 montre une plus grande diversité dans les types présents, alors qu'aucun élément modclé, si cc n'est un cordon, provient des locus 3 et 5 (tabl. XVI).

L. S.

\section{ANALYSE SPATIALE}

Malgré la faiblesse de certains effectifs (comme pour les formes) et la fouille partielle de certaines unités d'habitation (locus 4 et 6 notamment), l'analyse spatiale des principaux éléments typologiques, en nombre de vases, met en évidence des situations contrastées selon les locus (fig. 40). 
Tabl. XVI - Répartition des autres éléments modelés par locus.

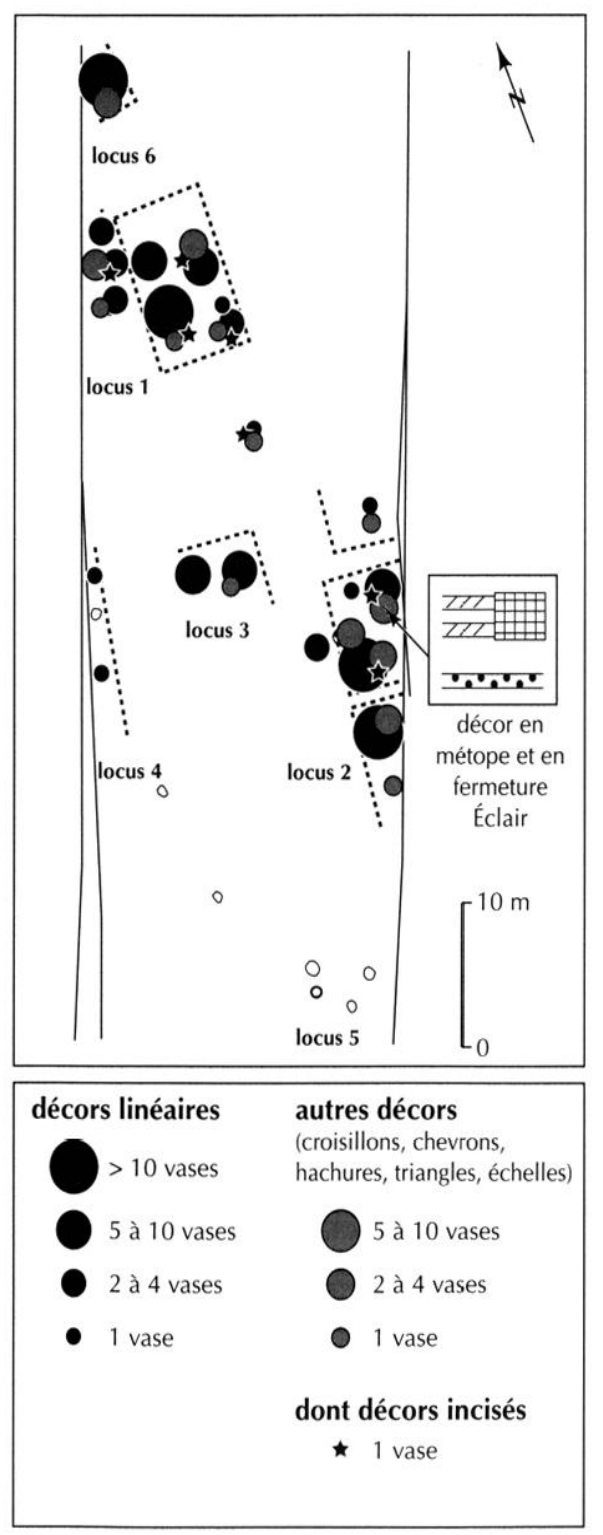

a

\begin{tabular}{|l|c|c|c|c|c|c|c|c|}
\hline & Anse * & Arrachement & Bouton & Cannelure & Perforation & Carène & Mamelon & Pied \\
\hline HS & 2 & & 1 & & & & & 1 \\
\hline Locus 1 & 3 & & & 4 & & & & \\
\hline Locus 2 & 1 & 2 & & 1 & & & & \\
\hline Locus 3 & & & & & & & & \\
\hline Locus 4 & 1 & & & & 1 & & & \\
\hline Locus 5 & & & & & & & & \\
\hline Locus 6 & 1 & & & & & 1 & 1 & 1 \\
\hline
\end{tabular}

*dont 2 à tenons dans locus 1
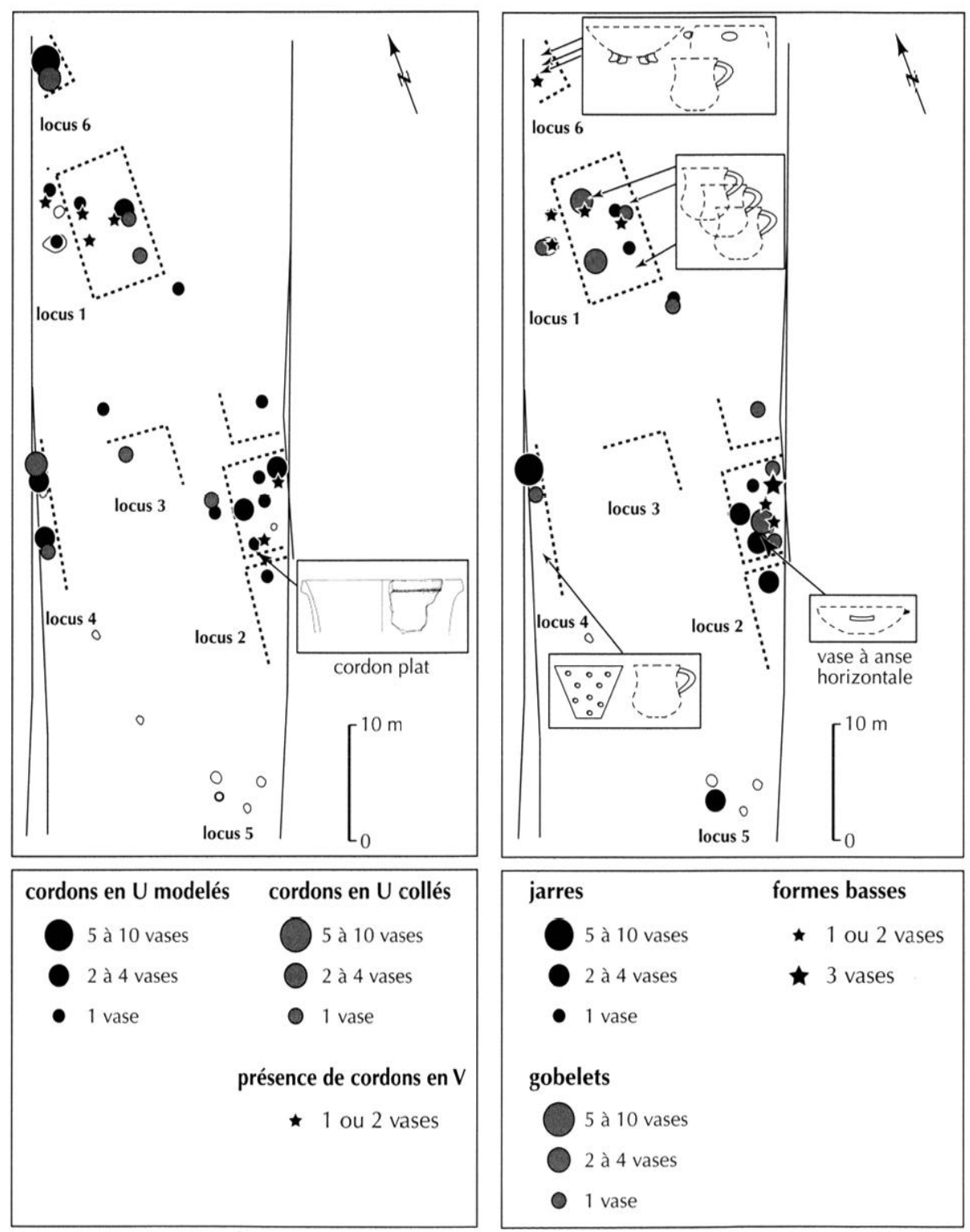

b

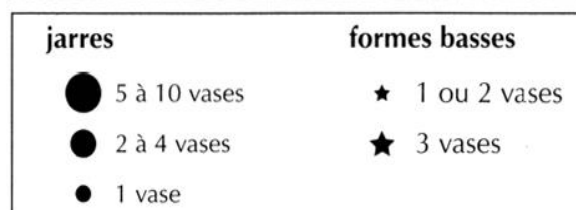

gobelets

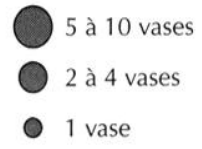

1 vase

Fig. 40 - Répartition par UF: a, des décors en creux; $b$, des cordons; $c$, des formes. 
En ce qui concerne les décors, leur répartition ne semble pas significative, si ce n'est que les deux décors les plus rares sur le site et les plus atypiques pour la région (métopes et "fermeture Éclair") proviennent du locus 2 (UF 27). La répartition des cordons en « $U$ » montre, quant à elle, qu'ils sont moins fréquents dans le locus 1 , alors que dans cette zone apparaît une relative fréquence des cordons en " V ». Là encore, l'unique exemplaire de cordon plat est issu du locus 2 (UF 16). La répartition des formes reconstituables confirme ces tendances: les grands vases de stockage (les jarres) sont assez rares dans le locus 1 qui regroupe essentiellement des vases auxquels on pourrait, de par leur forme, attribuer une fonction de préparation et de présentation culinaire (gobelets, cruches ou pichets, formes basses). La situation est identique dans le locus 6 qui comporte de surcroît des formes assez rares sur le site: coupe polypode et vase à mamelon. Le locus 2 comprend au contraire un grand nombre de vases de stockage et, une fois de plus, une forme rare et culturellement connotée: le vase à anse horizontale.

Nous constatons donc une différence significative entre les zones du site. Elles se matérialisent au niveau stylistique entre le locus 2 et les autres locus, celui-ci regroupant systématiquement les éléments les plus atypiques pour le site et pour la région. Le locus 6 manifeste les mêmes tendances, mais la fouille partielle de cette unité freine les tentatives de comparaison avec le locus 2. Au niveau fonctionnel, l'hypothèse d'une bipartition nord-sud du site peut également être émise : les vases de stockage sont concentrés au sud (locus 2 et 5 ), alors que les locus 1 et 6 comportent davantage de céramiques de service ou de préparation de l'alimentation.

Enfin, on ne peut écarter l'hypothèse d'un écart chronologique entre les locus, même si l'homogénéité technique de la série ne plaide pas en faveur d'un décalage important. Les indices de fragmentation sont identiques dans les locus 1 et 2. Il n'y a pas à ce niveau de différences, évoquant une plus grande érosion de l'une ou l'autre des unités. On pourrait envisager que les locus 2 et 6 soient relativement contemporains, car ils regroupent des éléments semblables, d'obédience extra-régionale. Rappelons toutefois que les liaisons entre tessons assurent la contemporanéité de fonctionnement des locus 1,2 et 4 . De même, les assemblages issus des fosses (UF 1 et UF 2 du locus 1, UF 13 du locus 2, UF 9 du locus 5) comportent des vases décorés et des céramiques communes variées, n'apportant aucun indice de chronologie relative. Cette contemporanéité de différents styles, déjà soulignée (Salanova, 2000), n'est de toute façon pas évidente à prouver dans un contexte de fosse dépotoir et d'unités d'habitation dont la durée d'utilisation nous échappe.

L. S.

\section{LES MATIÈRES PREMIÈRES}

\section{ET LES INCLUSIONS D'ORIGINE ANTHROPIQUE}

\section{Les matières premières}

Les déterminations pétrographiques ont été réalisées sur des lames minces de céramique, d'épaisseur normalisée et constante de $25 \mu \mathrm{m}$. Cette technique analytique est issue de la géologie. Son emploi est possible car la céramique est assimilable à une roche artificielle constituée de deux parties distinctes: d'une part d'une fraction initialement plastique appelée matrice et d'autre part d'une fraction non plastique correspondant aux inclusions essentiellement minérales. La détermination est réalisée essentiellement sur la fraction non plastique, la plus directement accessible au microscope car la matrice est composée de particules plus difficilement identifiables car de dimensions trop réduites $(<2 \mu \mathrm{m})$.

Le protocole d'étude et la description des lames minces ont été mis au point par L. Courtois (1971) et par J.-C. Échallier (1984), puis partiellement adaptés (Convertini, 1996). Ils ne seront donc pas redéfinis dans cette contribution et nous renvoyons le lecteur aux références citées ci-dessus.

\section{Détermination des groupes pétrographiques}

Suite à l'étude typotechnologique de la série de La Noue, des échantillons ont été prélevés sur 45 vases, choisis pour leur représentativité ou au contraire leur singularité.

À l'analyse microscopique, deux grandes familles de terres à l'origine des céramiques ont été reconnues. La première famille correspond à des argiles ne renfermant que des inclusions d'originc plutonique et métamorphique, essentiellement quartzo-micacées. La seconde famille regroupe des argiles recueillies en milieu sédimentaire, mais dont la majorité des constituants correspond néanmoins à ceux de la première famille.

Dans les deux cas, la même hiérarchisation est suivie. Tout d'abord, l'aspect de la matrice puis la densité en certaines particules traduisant l'état d'altération du cortège des inclusions non plastiques et, enfin, la présence ou l'absence de structure particulière sont à la base de la classification en groupes et sous-groupes. 
Première famille: argiles ne renfermant que des inclusions

d'origine plutonique et métamorphique (fig. 41)

Groupe A : à matrice aciculaive (vase $\mathrm{n}^{\circ} .36$ )

La matrice phylliteuse est constituée de minéraux se présentant sous forme d'aiguilles organisées entre elles et conduisant à une structure pseudo-lépidoblastique. Le mica noir est très abondant et constitue une grande partie des minéraux matriciels. Les autres inclusions sont peu abondantes. Elles sont émoussées à usées. Parmi elles, le quartz domine. Il est souvent granoblastique. Le feldspath potassique est relativement peu abondant. Les fragments de roches sont peu abondants. Quelques assemblages quartzofeldspathiques à structure granophyrique sont à noter.

Pour les autres céramiques, la matrice est toujours phylliteuse et cotonneuse, c'est-à-dire que les différents minéraux argileux ne sont pas perceptibles en microscopie optique.

\section{Groupe B: à micas noirs abondants}

Les inclusions sont émoussées. Le quartz domine toujours le cortège minéralogique. Le feldspath potassique est abondant, parfois perthitique. Le plagioclase est rare, mais toujours présent. Le mica blanc est rare, voire absent.

Ce groupe peut être scindé en trois en fonction de la proportion de fragments de roches d'origine plutonique. Dans tous les groupes, les coupures individualisant des sousgroupes ne sont pas clairement matérialisées. Il s'agit le plus souvent d'un continuum au sein duquel des ensembles d'individus se sont plus ou moins détachés.

- Sous-groupe I: à lithoclastes d'origine plutonique abondants :

a) avec assemblage quartzo-feldspathique à structure granophyrique (vase $\mathrm{n}^{\text {os }} 21$ et 35 ). Le mica noir est parfois décoloré. La zoïsite est présente dans la pâte du vase $n^{\circ} 21$. Le quartz est parfois granoblastique pour le vase $n^{\circ} 21$. Les inclusions sont abondantes dans les deux cas;

b) sans assemblage quartzo-feldspathique à structure granophyrique (vase $n^{\circ} 13$ ). La matrice est cotonneuse mais proche de l'aciculaire. Le mica noir est souvent décoloré. Les inclusions sont moyennement abondantes.

- Sous-groupe II: à lithoclastes d'origine plutonique moyennement abondants (vase $n^{\circ} 34$ ). Les inclusions sont également moyennement abondantes. Cet individu ne renferme pas d'assemblage à structure granophyrique.

- Sous-groupe III : à lithoclastes d'origine plutonique peu abondants. Une nouvelle subdivision permet de séparer les individus renfermant des assemblages quartzo-feldspa- thiques de ceux qui n'en renferment pas:

a) avec assemblage quartzo-feldspathique à structure granophyrique (vase $n^{\circ} 24$ ). Une épidote est présente. Les inclusions sont très abondantes et de taille essentiellement réduite;

b) sans assemblage quartzo-feldspathique à structure granophyrique (vase $n^{\circ} 12$ ).

Une amphibole verte est présente. Les inclusions sont peu abondantes.

Groupe C: à micas noirs moyennement abondants (vase $\mathrm{n}^{\circ} 6$ )

Les inclusions sont émoussées. Le quartz domine et de nombreux grains présentent une texture granoblastique. Le feldspath potassique est abondant tandis que le plagioclase est rare. Un microcline est présent. Le mica blanc est très rare. Les lithoclastes sont peu abondants.

Groupe D: à micas noirs peu abondants (vase $\mathrm{n}^{\circ} 10$ )

Les inclusions sont émoussées. Parmi elles, le quartz moyennement abondant domine suivi quantitativement par le feldspath potassique, parfois perthitique. Les autres particules sont toujours rares (plagioclases, micas blancs, lithoclastes). À noter la présence d'une tourmaline jaune.

Seconde famille: argiles renfermant des éléments d'origine sédimentaire (fig. 42)

Il s'agit dans tous les cas d'éléments calcédonieux (fibreux ou fibroradiés), de silexoïdes, parfois du quarty. " carié " dont l'origine se situe dans les terrains du Keuper (Trias). La matrice des céramiques est toujours phylliteuse et cotonneuse.

\section{Groupe E: à micas noirs abondants}

Les inclusions sont émoussées. Le quartz domine et le feldspath potassique est moyennement abondant. Le plagioclase reste très rare. Ces individus ne renferment pas d'assemblage quartzo-feldspathique à structure granophyrique.

- Sous-groupe I: à lithoclastes d'origine plutonique moyennement abondants (vase $n^{\circ} 3$ ). Le mica blanc est absent. Une fraction d'origine métamorphique est présente dans la pâte du vase $\mathrm{n}^{\circ} 3$.

- Sous-groupe II: à lithoclastes d'origine plutonique peu abondants (vase $n^{\circ} 45$ ). Le microcline est présent et le mica blanc est rare.

\section{Groupe F: à micas noirs moyennement abondants}

Les inclusions sont émoussées à usées. Le quartz domine toujours l'ensemble du cortège minéralogique. Le feldspath potassique est moyennement abondant tandis que le plagioclase est très rare. Les inclusions sont moyennement 


\section{PREMIÈRE FAMILLE : \\ ORIGINE PLUTONIQUE}

Éléments siliceux d'origine sédimentaire

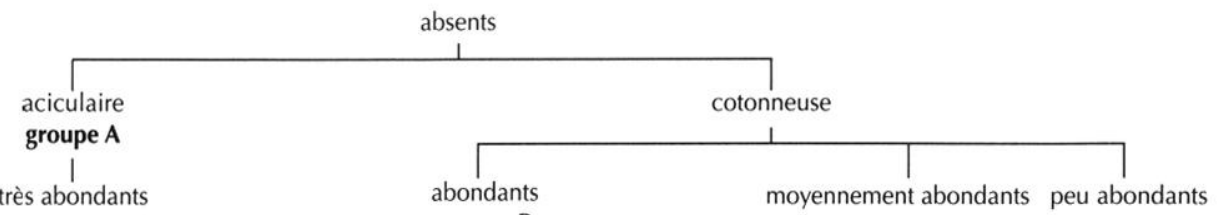

Aspect de la matrice
Micas noirs
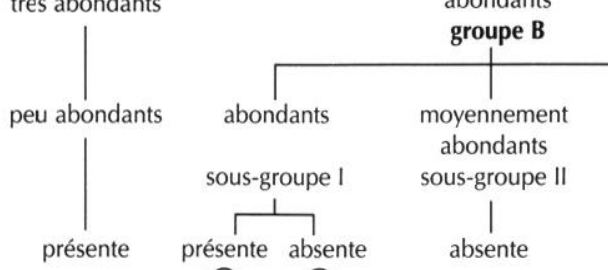

groupe C

Lithoclastes plutoniques

Structure granophyrique

présente

(a) (b)

Numéro de vase

36

35

34

peu abondants
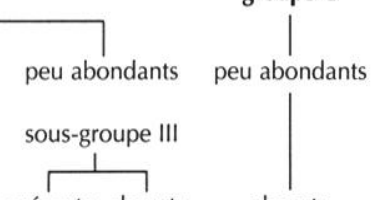

groupe $\mathbf{D}$

Fig. 41 - Classification des céramiques de la première famille à La Noue.

(a) (b)

$24 \quad 12 \quad 6$

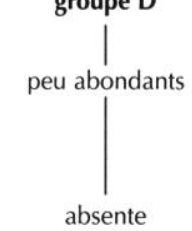

10

\section{SECONDE FAMILLE : ORIGINE SÉDIMENTAIRE}

Éléments siliceux d'origine sédimentaire

Aspect de la matrice

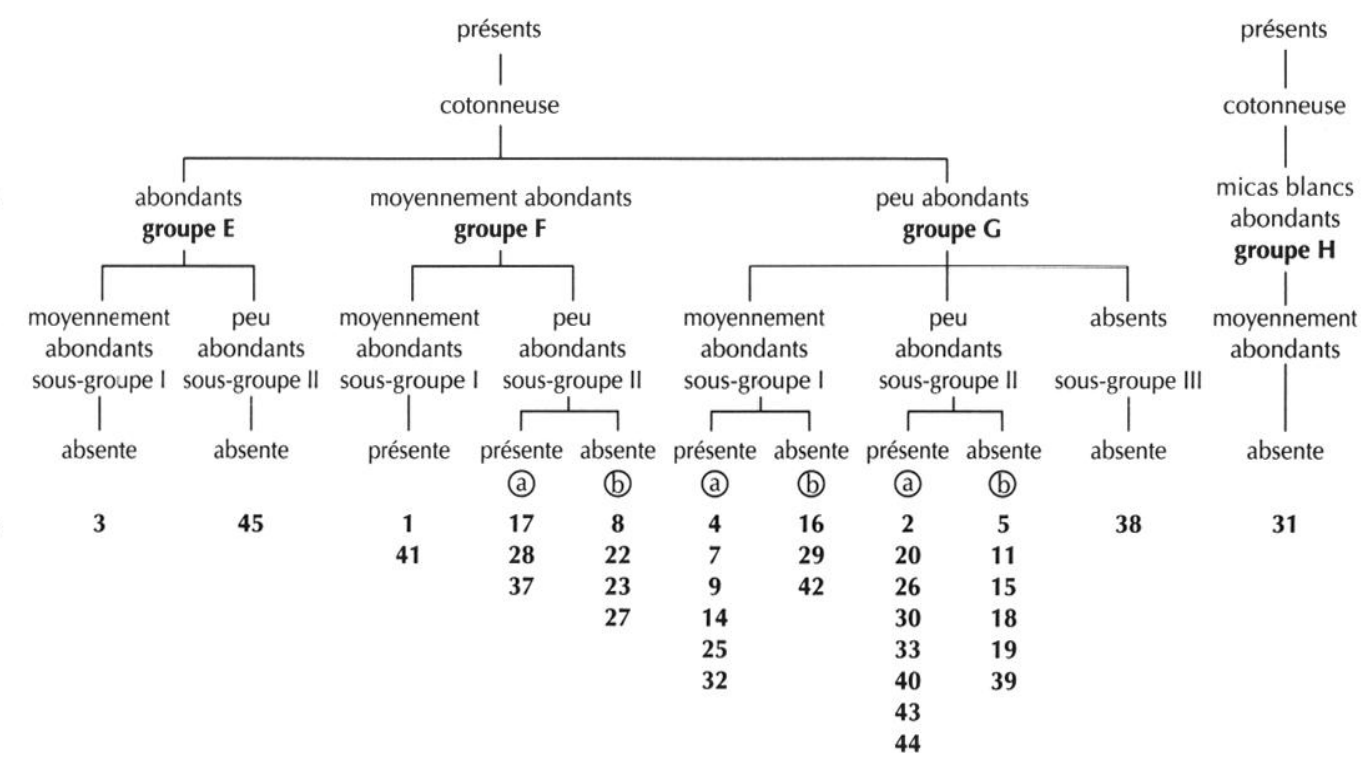

Fig. 42 - Classification des céramiques de la seconde famille à La Noue.

abondantes sauf pour le vase $\mathrm{n}^{\circ} 27$ pour lequel elles sont abondantes.

En fonction de la proportion de lithoclastes d'origine plutonique, ce groupe peut être subdivisé en deux.

- Sous-groupe I : à lithoclastes d'origine plutonique moyennement abondants (vases $n^{\text {os }} 1$ et 41 ). Les deux individus renferment des assemblages quartzo-feldspathiques à structure granophyrique. Le vase $n^{\circ} 1$ contient du micro- cline. Une épidote est présente dans la pâte du vase $n^{\circ} 41$. Les deux vases renferment une fraction de quartz à texture granoblastique.

- Sous-groupe II : à lithoclastes d'origine plutonique peu abondants. Une nouvelle subdivision peut être effectuée au sein de ce sous-groupe. Elle sépare les individus renfermant des assemblages à structure granophyrique de ceux n'en possédant pas: 
a) avec assemblage quartzo-feldspathique à structure granophyrique (vases $n^{\text {os }} 17,28$ et 37 ). Le microcline est présent dans la pâte du vase $\mathrm{n}^{\circ} 17$ et une tourmaline verte dans celle du vase $\mathbf{n}^{\circ} 37$. Quelques quartz à texture granoblastique sont présents dans la pâte du vase $\mathrm{n}^{\circ} 28$;

b) sans assemblage quartzo-feldspathique à structure granophyrique (vases $\mathrm{n}^{\text {os }} 8,22,23$ et 27 ). Une tourmaline verte est présente dans la pâte du vase $\mathrm{n}^{\circ} 23$. Les vases $\mathrm{n}^{\text {os }} 22$ et 27 renferment de rares quartz " cariés ". Quelques quartz à texture granoblastique sont présents dans les quatre céramiques. À noter que la pâte des vases $\mathrm{n}^{\mathrm{os}} 8,23,28$ et 37 , hormis la présence de quelques éléments à structure granophyrique pour les deux derniers, est similaire.

\section{Groupe G: à micas noirs peu abondants}

Les inclusions sont émoussées à usées. Parmi elles, le. quartz est dominant. Le feldspath potassique est moyennement abondant ou rare. Le plagioclase est toujours très rare ou absent. Les micas noirs et blancs sont toujours présents mais en faible quantité. La calcédoine est quasiment présente dans toutes les pâtes, souvent sous forme fibroradiée. Le silex est plus rare.

Ce groupe peut être subdivisé en fonction de la densité relative des lithoclastes d'origine plutonique.

- Sous-groupe I: à lithoclastes d'origine plutonique moyennement abondants. La subdivision correspondant à la séparation entre présence et absence d'assemblage quartzo-feldspathique à structure granophyrique est reconduite:

a) avec assemblage quartzo-feldspathique à structure granophyrique (vases $n^{\text {os }} 4,7,9,14,25$ et 32 ). Une tourmaline verte est présente dans la pâte du vase $\mathrm{n}^{\circ} 32$. Les individus $\mathrm{n}^{\text {os }} 7$ et 9 renferment du quartz originaire du Trias. La pâte du vase $n^{\circ} 4$ renferme un lithoclaste d'origine plutonique plus basique que les autres (origine granodioritique). Les lithoclastes d'origine métamorphique sont toujours présents mais en faible quantité (quartzite, gneiss). La densité en inclusions est variable : forte (vases $\mathrm{n}^{\circ} 4,9,14$ et 32 ), moyennement forte (vase $\mathrm{n}^{\circ} 7$ ) et faible (vase $\mathrm{n}^{\circ} 25$ ). Les céramiques $n^{o s} 4,9,14$ et 32 se caractérisent par une densité importante en inclusions de taille réduite. Leurs pâtes sont proches tout en étant néanmoins distinctes;

b) sans assemblage quartzo-feldspathique à structure granophyrique (vases $\mathrm{n}^{\text {os }} 16,29$ et 42 ). Les trois vases renferment du quartz " carié ". Les inclusions sont peu abondantes pour le vase $\mathrm{n}^{\circ} 16$ et moyennement abondantes pour les deux autres céramiques.

- Sous-groupe II: à lithoclastes d'origine plutonique peu abondants. Au sein de ce groupe, une autre subdivision peut être introduite séparant les vases qui renferment des assemblages quartzo-feldspathiques à structure granophyrique de ceux qui n'en contiennent pas:

a) avec assemblage quartzo-feldspathique à structure granophyrique (vases $\mathrm{n}^{\mathrm{os}} 2,20,26,30,33,40,43$ et 44 ). Une tourmaline jaune est présente dans la pâte du vase $n^{\circ} 44$. Une amphibole verte et une zoïsite complètent le cortège minéralogique du vase $n^{0} 43$. Quelques grains de quartz " carié " sont présents dans deux vases ( ${ }^{\text {os }} 20$ et 33 ). Quelques fragments de roches métamorphiques sont parfois présents (vases $\mathrm{n}^{\text {os }} 2$ et 26 ), le quartz étant quelquefois à texture granoblastique. La densité en inclusions est variable selon les individus. Elles sont abondantes (vases $\mathrm{n}^{\text {os }} 20,26$ et 44), moyennement abondantes (vases $\mathrm{n}^{\text {os }} 2$ et 33) et peu abondantes (vases $n^{\text {os }} 30,40$ et 43 ). Les vases $\mathrm{n}^{\text {os }} 20,26$ et 44 se caractérisent par une densité importante en inclusions de taille réduite. Les vases ${ }^{\text {os }} 30,40$ et 43 sont similaires tout comme les vases $\mathrm{n}^{\text {os }} 2$ et 33 et les vases $\mathrm{n}^{\text {os }} 20$, 26 et 44 . Signalons que le vase $\mathrm{n}^{\circ} 33$ renferme de rares quartz triasiques contrairement au $\mathrm{n}^{\circ} 2$;

b) sans assemblage quartzo-feldspathique à structure granophyrique (vases $n^{\text {os }} 5,11,15,18,19$ et 39 ). Trois vases $\left(\mathrm{n}^{\text {os }} 5,11\right.$ et 19) renferment quelques quartz " cariés ". Les lithoclastes d'origine métamorphique sont parfois présents en faible quantité (vases $\mathrm{n}^{\text {os }} 15$ et 19). De plus, quelques grains de quartz montrent une texture granoblastique (vases $\mathrm{n}^{\text {os }} 11$ et 39). La densité en inclusions varie selon les individus: moyennement forte pour les vases $\mathrm{n}^{\mathrm{os}} 15,19$ et 39 et faible pour les vases $n^{\text {os }} 5,11$ et 18 . Hormis la présence de lithoclastes à structure granophyrique et du quartz triasique, la pâte du vase $n^{\circ} 5$ est similaire à celle des vases $n^{\text {os }} 30,40$ et 43 du sous-groupe IIa ainsi que du vase $\mathrm{n}^{\circ} 25$ de Ia. De la même façon, le vase $\mathrm{n}^{\circ} 39$ est très proche des vases $n^{\text {os }} 2$ et 33 .

- Sous-groupe III : à lithoclastes d'origine plutonique absents (vase $\mathrm{n}^{\circ} 38$ ). Les inclusions sont peu abondantes. Une épidote a été observée. Un quartz est d'origine triasique.

Groupe $H$ : à micas blancs abondants (vase $\mathrm{n}^{\circ} 31$ )

La présence de micas blancs en grande quantité individualise ce vase qui ne se démarque pas, par ailleurs, des autres groupes de la seconde famille de pâte. La matrice est phylliteuse et cotonneuse.

Les inclusions sont émoussées à usées. Le quartz domine le cortège minéralogique parmi lequel figure aussi le feldspath potassique moyennement représenté et le très rare plagioclase. Le mica noir est également présent. Beaucoup moins abondant que le blanc, il est néanmoins 
également associé aux autres minéraux dans les lithoclastes d'origine plutonique. Ces lithoclastes ont une proportion moyennement forte au sein des inclusions. Quelques quartz présentent une texture granoblastique. Des tourmalines brunes et jaunes sont présentes.

Il faut souligner tout de suite les limites du classement en deux grandes familles basé sur la présence ou l'absence d'éléments sédimentaires qui sont le plus souvent présents en très faible quantité. En effet, le silex et la calcédoine, mais également les quartz triasiques, ne sont parfois représentés que par un seul individu dans la surface étudiée en lame mince. Cette situation est particulièrement gênante lorsque les particules non-plastiques sont rares. Heureusement, ce cas extrême ne concerne qu'un seul individu rangé dans groupe $B$, sous-groupe IIIb (vase $n^{\circ} 12$ ). Il existe donc une possibilité non négligeable pour ce vase de ne pas avoir été classé dans la famille d'argiles appropriée. Pour les autres céramiques, la quantité d'inclusions est beaucoup plus importante et le risque d'erreur est moindre. En toute rigueur, ce sont donc huit individus céramiques ( $\mathrm{n}^{\text {os }} 6,10,12,13,21,24,34$ et 35 ) appartenant aux groupes B, C et D qui sont susceptibles d'appartenir à la seconde famille et de passer dans les groupes $\mathrm{E}, \mathrm{F}$ et $\mathrm{G}$.

De la même façon, mais avec des conséquences beaucoup plus limitées sur le regroupement des vases, les céramiques qui ne montrent pas de lithoclastes présentant une structure granophyrique visibles dans le champ de la lame mince pourraient ne pas être à leur place à l'intérieur des différents sous-groupes individualisés. En effet, ces éléments sont toujours présents en faible quantité et leur absence dans la surface de la lame mince ne signifie pas forcément une réelle absence dans l'argile exploitée.

\section{Propositions de provenances possibles des ressources argileuses dans un cadre régional}

\section{Groupe A}

Le seul individu rattaché à ce groupe possède une matrice fortement aciculaire très riche en micas noirs. La terre utilisée ne peut que correspondre à une altérite peu évoluée de granite à biotite probablement prélevée in situ. Les terrains les plus proches correspondent à la dorsale cristalline du Charollais présente à l'ouest du site, dans la partie orientale du Massif central (Koch et al., 1976; Courel et al., 1989). Cette dorsale appelée horst de Mont-Saint-Vincent comprend essentiellement un axe de gneiss et deux domaines granitiques orientés nord-est - sud-ouest. Le granite le plus à l'est est un granite calco-alcalin à biotite. Dénommé granite de Mary, il s'agit de la formation la plus proche, localisée à une vingtaine de kilomètres à l'ouest du site, dont la description correspond à l'ensemble des caractères observés, y compris la présence de lithoclastes à structure granophyrique. Bien entendu, d'autres granites à biotite existent sur la bordure orientale du Massif central depuis le Vivarais au sud jusqu'au Morvan au nord. Seule une analyse chimique permettrait de localiser avec certitude la roche-mère.

\section{Groupe B}

L'abondance des micas noirs contenus dans la pâte des vases de ce groupe indique la proximité de formations riches en biotites. La dorsale cristalline du Charollais est constituée de granites, dont l'un est probablement à l'origine des altérites du groupe $\mathrm{A}$, et des roches gneissiques renfermant également de la biotite. La position exacte des dépôts argileux exploités est difficile à situer, mais ils ne peuvent pas être éloignés de ces terrains. Par rapport à la terre du groupe A, les argiles du groupe B présentent des différences comme l'aspect cotonneux de la matrice, la présence sporadique de micas blancs et de lithoclastes d'origine métamorphique. Mais les assemblages quartzofeldspathiques à structure granophyrique et plusieurs caractéristiques minéralogiques sont identiques à celles du vase du groupe A. La même roche-mère granitique a donc servi, en partie, à alimenter les dépôts argileux à l'origine des céramiques, mais la présence de muscovite et de débris de roches d'origine métamorphique implique un apport depuis une ou plusieurs autres sources, probablement gneissiques. La notice de la carte géologique de Montceau-lesMines signale des formations d'altération pouvant atteindre $20 \mathrm{~m}$ d'épaisseur sur les affleurements de roches cristallines et métamorphiques de la dorsale du Charollais. Ces épais niveaux correspondent à des sables argileux micacés, lesquels ont pu être, pour les plus argileux d'entre eux, à l'origine des céramiques rangées dans ce groupe. Les argiles du groupe B correspondent à un état d'altération plus avancée des micas noirs que celles du groupe A. La densité en lithoclastes est également fonction du degré de fragmentation et d'altération de la roche-mère et les différents sousgroupes ne font que traduire ces variations. Il s'agit chaque fois de prélèvements effectués dans une zone d'altération différente, donc nécessairement dans des lieux géographiques distincts. 


\section{Groupe C}

Tout comme les vases du groupe précédent, la seule céramique de ce groupe a été fabriquée avec une argile provenant essentiellement de l'altération d'une roche plutonique renfermant du mica noir. La proportion de ce minéral est ici moindre que pour les individus du groupe B. En dépit du fait qu'il n'y ait pas de lithoclastes à structure granophyrique, toujours rares dans les céramiques des autres groupes, ni de mica blanc, une origine similaire peut être néanmoins proposée. La dégradation des constituants micacés et la désagrégation des fragments de roches sont plus importantes que pour les argiles du groupe $\mathrm{B}$.

\section{Groupe D}

Les proportions de micas noirs et de lithoclastes diminuent encore par rapport aux groupes précédents. Cette terre est le résultat d'une altération poussée de la roche-mère, la plus intense de cette première famille. Le cortège minéralogique restant identique à celui des groupes précédents, renfermant du mica blanc et une faible quantité d'éléments métamorphiques, il est logique de proposer la même origine pour cette argile.

L'altération progressive observée entre les individus du groupe B et le vase du groupe $\mathrm{D}$ n'est probablement pas liée à un phénomène d'éloignement de la source émettrice. En effet, si c'était le cas, la probabilité de voir apparaitre dans les argiles utilisées des éléments étrangers à la minéralogie du dépôt-source augmenterait au fur et à mesure de la distance. Or, le stock minéralogique reste constant et aucune particule de type sédimentaire n'a été observée dans les lames minces des vases des groupes $\mathrm{C}$ et $\mathrm{D}$ qui renferment une quantité relativement importante d'inclusions, surtout en ce qui concerne le premier ${ }^{8}$. Il semble plutôt que la disparition des minéraux fragiles et la fragmentation des lithoclastes ne résultent pas d'une action physique à la suite d'un déplacement qui les ferait disparaître, mais s'effectuent in situ par des phénomènes poussés d'hydrolyse.

Les pâtes des céramiques de la seconde famille renferment toutes des inclusions d'origine sédimentaire, toujours très rares au sein de l'ensemble du cortège. Ces particules ne correspondent jamais à des carbonates clairement identifiés. Néanmoins, un possible cas est à envisager car la pâte du vase $n^{\circ} 29$ présente des vacuoles partiellement comblées par des particules jaunâtres en lumière naturelle et totale-

8. Néanmoins, il faut garder à l'esprit les limites du classement développées dans le paragraphe précédent. ment isotropes en lumière polarisée. Ces éléments peuvent correspondre à des carbonates ayant subi une température de cuisson élevée qui aurait endommagé la structure des cristaux. Hormis ce cas douteux, aucune autre argile utilisée pour la fabrication de vase ne semble contenir d'inclusions carbonatées.

La carte géologique simplifiée de la région chalonnaise (fig. 43), réalisée à partir des cartes au 1/50000 de Chalonsur-Saône, Chagny, Montceau-les-Mines et Le Creusot, permet de visualiser les différents domaines pétrographiques. À l'ouest, affleurent les terrains paléozoïques et le socle cristallin qui est localement recouvert par des dépôts du Secondaire (Trias et Jurassique) (Koch et al., 1976; Courel et al., 1989). Cette zone correspond à la Côte chalonnaise.

La totalité des formations meubles granulométriquement fines pouvant être à l'origine des céramiques de $\mathrm{La}$ Noue sont situées en rive droite de la Saône. En effet, les apports issus du cristallin, du Trias et les éléments siliceux d'origine sédimentaire se concentrent sur les versants de la Côte chalonnaise, dans les alluvions et aux débouchés des cours d'eau tributaires de la Saône provenant des monts du Charollais. Aucune formation de la rive gauche, et plus généralement de la Bresse, ne peut être à l'origine de la totalité du cortège pétrographique mis en évidence. Bien sûr, les quartz " cariés " sont présents dans les alluvions du Doubs, affluent de rive gauche de la Saône et rivière qui traverse des formations du Trias argileux dans le massif du Jura. De la même façon, les éléments siliceux (silex et chailles) sont des constituants de beaucoup d'autres roches sédimentaires du bassin de la Saône, mais les micas noirs et les éléments quartzo-feldspathiques, dont certains présentent une structure granophyrique, sont absents de ces contextes sédimentaires et seulement présents dans les formations de rive droite.

Parmi les tributaires de rive droite, les alluvions de la Grosne paraissent réunir toutes les caractéristiques requises, en grande partie grâce à son affluent de rive gauche, la Guye. Le bassin versant de l'Orbise draine des formations variées (granitiques, métamorphiques, gneiss et micaschistes, gréseuses, argileuses et carbonatées), mais le cours d'eau ne traverse pas directement le granite à biotite. Néanmoins celui-ci peut être remanié dans les niveaux du Trias mais, dans ce cas, seules les alluvions anciennes affleurant en amont du cours d'eau pourraient correspondre à certaines argiles de la seconde famille. Le bassin versant de la Dheune draine les formations sédimentaires du Primaire, 


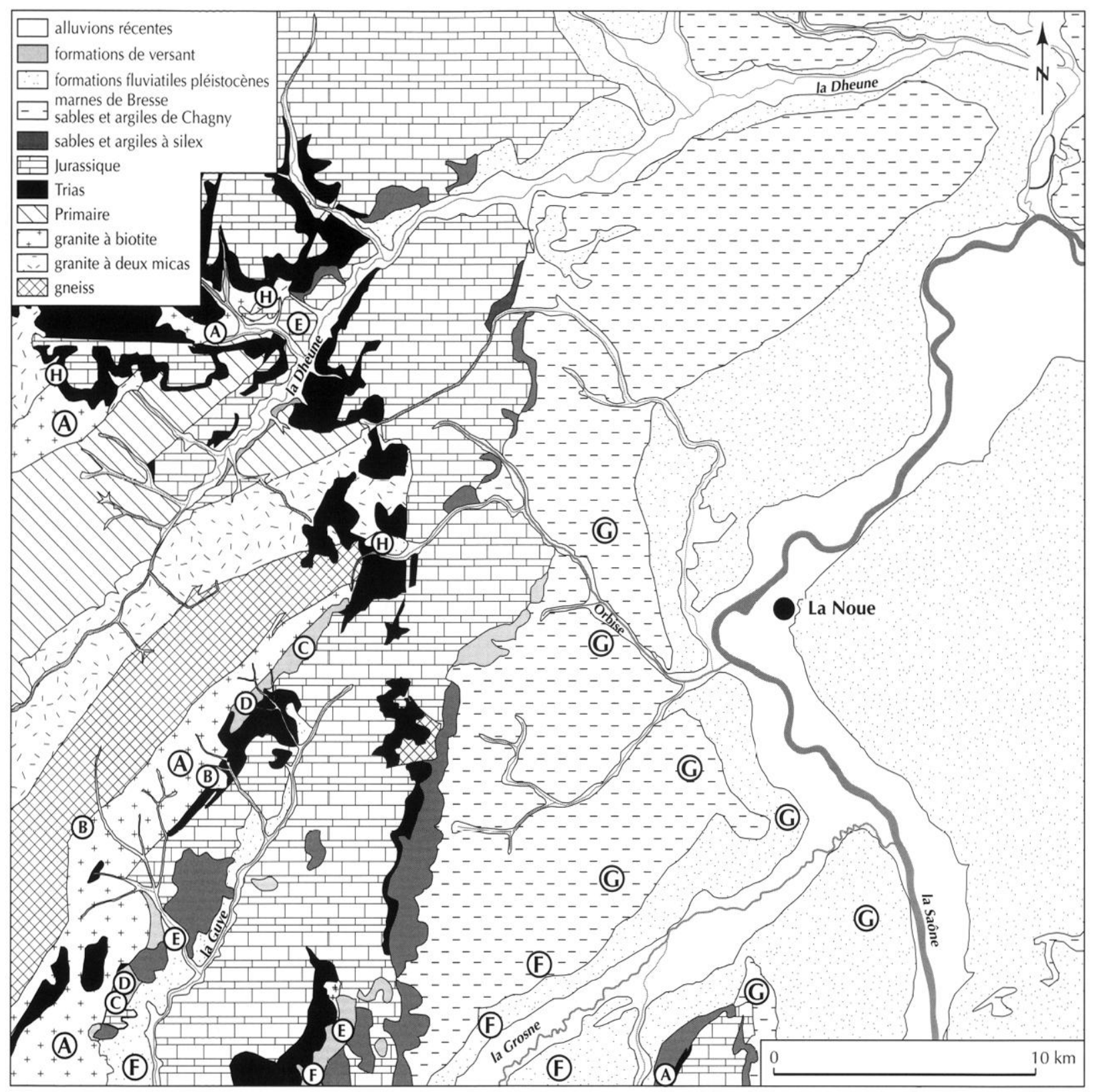

Fig. 43 - Localisations potentielles des argiles utilisées pour la fabrication des céramiques de La Noue (carte F. Convertini).

les gneiss, le granite à deux micas et, par ses tributaires de rive gauche, le granite à mica noir.

Il est malheureusement impossible de localiser avec précision l'ensemble des affleurements présentant les caractéristiques reconnues en lame mince. Comme pour les argiles de la première famille, seules seront indiquées les potentialités dans le cadre de la carte sur une surface voisine de $1500 \mathrm{~km}^{2}$ (fig. 43). Bien entendu, ce périmètre pourrait être étendu vers le sud en direction des monts du Mâconnais, dont l'extrémité septentrionale affleure au bas de la carte.

\section{Groupe E}

Comme pour la première famille, l'abondance en micas noirs indique la proximité de formations riches en biotite. Ce minéral étant relativement fragile, il est peu vraisemblable qu'il ait été transporté sur une grande distance. Par conséquent, les affleurements doivent être localisés sur le plateau aux abords de formations libérant de la biotite. Les zones potentielles se situent dans les niveaux fluvio-lacustres de la vallée de la Guye ou, plus à l'est, dans les niveaux colluviaux alimentés par le Trias et les roches granitiques à 
biotite ainsi que dans la vallée de la Dheune. L'échantillon $\mathrm{n}^{\circ} 3$ renferme un fragment de coquille silicifiée.

\section{Groupe F}

Par rapport au groupe précédent, l'abondance en micas noirs décroît, résultant d'altérations chimiques ou physiques dues au transport. Le cortège pétrographique reste toujours le même dans des proportions variables.

À ce stade de la recherche des argiles à l'origine des céramiques, l'emploi de niveaux fins alluviaux ou fluviolacustres doit être envisagé. Les dépôts les plus anciens, situés au pied de la Côte chalonnaise, correspondent à la formation des marnes de Bresse et des sables et argiles de Chagny qui est constituée essentiellement de quartz et de calcites. De façon générale, cette formation, puis les dépôts postérieurs d'origine fluviatile de la vallée de la Saône ne sont pas à retenir comme un ensemble potentiel sauf au débouché des cours d'eau de rive droite, où ils présentent des caractères sensiblement différents. En effet, ces trois cours d'eau, la Dheune, l'Orbise et la Grosne descendent du Massif central et leurs alluvions anciennes renferment pour partie une fraction d'origine granitique. Néanmoins, dans l'étendue de la carte, seule la Grosne, dans laquelle se jette la Guye, et dans une moindre mesure l'Orbise possèdent un bassin versant renfermant la totalité des éléments observés. Les carbonates sont totalement absents des alluvions contrairement à la Dheune. La relative proportion de mica noir semble exclure néanmoins l'Orbise. Par conséquent, les niveaux fins de la formation des marnes de Bresse et des dépôts fluviatiles postérieurs situés à proximité du débouché de la Grosne, mais également ceux des propres alluvions de ce cours d'eau, ont pu être potentiellement exploités par les Campaniformes.

\section{Groupe G}

Les remarques précédentes sont également valables pour ce groupe qui présente des terres encore plus pauvres en micas noirs. Les niveaux exploités sont probablement localisés dans les cônes alluviaux de la Grosne et peut-être, dans ce cas, de l'Orbise.

Deux vases renferment respectivement un fragment de spongiaire calcédonieux $\left(\mathrm{n}^{\circ} 15\right)$ et un fragment de calcaire silicifié ( $\left.n^{\circ} 43\right)$.

Ce groupe contient plus de la moitié des céramiques analysées. Les niveaux exploités sont, a priori, les plus proches de La Noue.

\section{Groupe $H$}

La minéralogie du seul vase de ce groupe ne s'écarte de celle qui a été observée dans les autres groupes que sur un point: l'abondance de micas blancs. Les autres inclusions correspondent aux micas noirs sous forme libre ou en association dans des lithoclastes d'origine plutonique. Les éléments siliceux (silex et calcédoine fibreuse) sont également présents.

L'origine de la terre employée pour la fabrication de ce vase est à rechercher dans des formations sédimentaires granulométriquement fines renfermant à la fois des éléments en provenance d'un granite à biotite et d'autres issus d'une roche riche en muscovite pouvant être également granitique ou d'origine métamorphique. La présence de biotite indique que ce dépôt se situe en rive droite de la Saône sans qu'il soit possible de le localiser. À titre d'hypothèse, il peut correspondre à une vieille terrasse non loin du granite à biotite, des gneiss et des affleurements triasiques. Malgré la difficulté de situer la terre qui a servi à la fabrication de ce vase, il sera provisoirement classé dans les argiles de la seconde famille renfermant des éléments d'origine sédimentaire.

En conclusion, les groupes et les sous-groupes de chaque famille rassemblent des céramiques fabriquées à partir de terres argileuses issues de l'altération différenciée de roches-mères. Le stade d'altération varie notamment en fonction de la distance existant entre les roches et les altérites. Plus cette distance est grande, plus l'altération est importante et plus la proportion en minéraux fragiles décroît. Les terres employées pour la confection des céramiques de La Noue montrent cette évolution. Elles semblent correspondre majoritairement aux alluvions de plusieurs cours d'eau (la Saône et ses affluents de rive droite). L'aspect toujours lenticulaire de ces dépôts est souligné par les auteurs des levés de la carte géologique, ce qui implique qu'il est quasiment impossible de retrouver les positions précises des affleurements exploités. Ces conditions de gisement expliquent pourquoi, dans un même type d'environnement, les argiles utilisées pour la fabrication des vases par les Campaniformes sont aussi hétérogènes. Seuls les individus des sous-groupes GI et GII montrent des similitudes, ce qui peut s'expliquer par le fait qu'ils ont été confectionnés à partir de dépôts argilo-sableux relativement homogènes et d'extension plus vaste. 


\section{Analyse spatiale}

Le locus 1 a livré deux structures qui recoupent le niveau archéologique. La première UF 1 renfermait du mobilier très fragmenté. Quatre échantillons analysés $\left(\mathrm{n}^{\circ \mathrm{os}} 6,7,8\right.$ et 9$)$ proviennent de cette fosse qui est interprétée comme dépotoir. Ils correspondent à des vases classés respectivement dans les groupes et sous-groupes C, GIa, FIIb et GIa, c'est-à-dire dans les deux familles de terres. La deuxième fosse UF 2 a livré essentiellement les restes de deux céramiques en bon état qui ont fait l'objet d'une analyse ( $\mathrm{n}^{\text {os }} 10$ et 37 ). Elles sont respectivement rangées en $\mathrm{D}$ et FIIa et proviennent également des deux familles d'argiles. Les autres céramiques étudiées du locus $1\left(\mathrm{n}^{\mathrm{os}} 5\right.$, $11,12,13,14,15,16,17,18,19,39,40,41,43$ et 45 ) proviennent du niveau de sol et ont été fabriquées pour la plupart avec des argiles de la seconde famille, seuls deux vases ( $\mathrm{n}^{\text {os }} 12$ et 13$)$ ayant été confectionnés avec des terres du groupe B (IIIb et Ib). Sur l'ensemble du locus 1, quatre vases sont rangés dans la première famille et dix-sept dans la deuxième.

Le locus 2 a également livré deux structures en creux. Il s'agit d'un trou de poteau (UF 44) et d'une fosse dont le contenu est interprété comme dépotoir (UF 13). Trois individus $\left(\mathrm{n}^{\text {os }} 2,3\right.$ et 4$)$ de cette dernière structure ont été analysés. Ils sont respectivement classés en GIIa, EI et GIa, soit tous trois dans la seconde famille. Treize autres tessons du locus 2 ont été soumis à l'analyse ( $\mathrm{n}^{\mathrm{os}} 20,21,22,23,24,25$, $26,27,35,36,38,42$ et 44). Parmi eux, quatre appartiennent à la première famille de terres $\left(\mathrm{n}^{\mathrm{os}} 21,24,35\right.$ et 36$)$. Il s'agit d'argiles du groupe $\mathrm{A}\left(\mathrm{n}^{\circ} 36\right)$ et de $\mathrm{B}$ (BIa pour $\mathrm{n}^{\mathrm{os}} 21$ et 35 ; BIIIa pour $\left.n^{\circ} 24\right)$. Ce locus a également livré des céramiques regroupées dans les deux familles (quatre dans la première et douze dans la deuxième).

Aucun tesson du locus 3 n'a été analysé.

Les deux céramiques du locus 4 étudiées en lame mince sont classées dans la deuxième famille, en FIIa $\left(\mathrm{n}^{\circ} 28\right)$ et (SIb $\left(n^{\circ} 29\right)$.

La céramique à demi enterrée (UF $9 ; n^{\circ} 1$ ) du locus 5 a été fabriquée avec une argile de FI.

Dans le locus 6, les trois vases analysés sont classés dans la seconde famille, deux d'entre eux en $\mathrm{G}$ (Ia pour le $\mathrm{n}^{\circ} 32$ et IIa pour le $\left.\mathrm{n}^{\circ} 30\right)$ et le dernier en $\mathrm{H}\left(\mathrm{n}^{\circ} 31\right)$.

Dans les locus 1 et 2, numériquement les plus riches en individus analysés, les céramiques ont été fabriquées indifféremment avec les deux grandes familles de terres sans qu'apparaissent d'espaces particuliers pour lesquels l'une ou l'autre des origines serait exclusive.
Des vases confectionnés avec diverses sources argileuses sont présents dans la même nappe de vestiges. Si chaque nappe correspond à l'emplacement d'une unité d'habitation, d'une durée de vie relativement courte, alors tous les vases ont été utilisés (et cassés) dans un intervalle de temps réduit. Deux observations peuvent étayer cette hypothèse. Tout d'abord, la forte similitude de pâte de trois individus ( $\mathrm{n}^{\mathrm{os}} 20$, 26 et 44) regroupés en GIIIa et recueillis dans le même locus 2 et ensuite l'assemblage céramique contenu dans le comblement de la fosse 1 qui, s'il est bien de formation rapide, met en synchronie des vases fabriqués avec des argiles de trois groupes représentant les deux familles de terres.

La contemporanéité entre les différents locus est plus difficile à assurer bien que trois vases de composition similaire proviennent de trois locus différents 1,2 et 6 . La nature lenticulaire et variée des dépôts exploités est un frein à toute tentative de rapprochement.

\section{Confrontation des données pétrographiques avec les données issues des autres analyses céramologiques}

Les échantillons soumis à l'analyse pétrographique ont été choisis à la fois parmi la céramique commune (23 individus) et les vases décorés (22 individus), soit une proportion équilibrée des deux ensembles.

Du fait de la grande dispersion des vases dans plusieurs groupes et sous-groupes, l'analyse se bornera le plus souvent à chercher des corrélations au niveau du sous-groupe, ou du groupe dans le cas d'ensembles numériquement faibles (tabl. XVII).

\section{Avec la nature de la céramique commune/décorée et la morphotypologie}

Parmi la première famille, seul le groupe B renferme plusieurs individus, au nombre de six. Parmi eux, un seul exemplaire correspond à un gobelet décoré $\left(\mathrm{n}^{\circ} 24\right)$. Quatre autres céramiques communes, dont au moins deux vases correspondant à des jarres à cordon préoral ( ${ }^{\text {os }} 12$ et 21$)$, sont présentes. L'une d'entre elles $\left(\mathrm{n}^{\circ} 21\right)$ porte une ligne de coups de poinçon. Un autre tesson présente un décor réalisé à la spatule $\left(n^{\circ} 13\right)$. Une anse en boudin ( $\left.n^{\circ} 35\right)$ fait partie d'un vase dont la forme est inconnue. Enfin, un pied ( $\left.n^{\circ} 34\right)$ appartient à une coupe polypode dont on ne sait pas si elle portait un décor. Le sous-groupe $\mathrm{BI}$, numériquement le plus étoffé, ne renferme que de la céramique commune. Avec le seul gobelet décoré du groupe $\mathrm{A}$, ces six vases ont été fabriqués à partir d'argiles proches de roches riches en micas noirs situées au moins à une vingtaine de kilomètres de La Noue. Ce cas de 
Tabl. XVII - Récapitulatif des données pétrographiques de la céramique analysée.

\begin{tabular}{|c|c|c|c|c|}
\hline $\mathrm{N}^{\circ}$ Éch. & Pétro. & $\mathrm{N}^{\circ}$ dessin & Type de vase - Techniques et thèmes décoratifs & Répartition spatiale \\
\hline 2 & Gilla & fig. 26-10 & bord de gobelet non décoré & locus 2-UF $13-205$ \\
\hline 4 & Gla & fig. $25-6$ & bandes hachurées au peigne & locus 2-UF 13 - 207 \\
\hline 5 & Gllb & fig. 14-7 & lignes horizontales au peigne & locus 1-UF $01-316$ \\
\hline 7 & Gla & fig. $14-2$ & vase tonnelet à cannelure sous le bord & locus 1-UF $01-319$ \\
\hline 8 & FIllb & fig. 14-5 & cordon préoral & locus 1-UF $01-317$ \\
\hline 9 & Gla & fig. $14-8$ & lignes horizontales au peigne & locus 1-UF 01 - 315 \\
\hline 10 & $\mathrm{D}$ & fig. 14-3 & bandes de lignes horizontales et triangles rayés au peigne & locus 1-UF $02-199$ \\
\hline 11 & Gllb & fig. 18-9 & cordon préoral & locus 1-UF 19 - 264 et 267 \\
\hline 15 & Gllb & fig. $15-15$ & croisillons au peigne & locus 1 -UF $03-217$ \\
\hline 16 & Glb & fig. 16-5 & lignes horizontales au peigne & locus 1 -UF 18 - 276 \\
\hline 17 & Flla & fig. 17-12 & triangles rayés au peigne & locus 1-UF 19 - 34 \\
\hline 18 & Gllb & fig. 15-4 & anse en boudin à tenon & locus 1-UF 15 - 161 \\
\hline 19 & Gllb & fig. 19-7 & lignes horizontales au peigne & locus 1 -UF $22-230$ \\
\hline 20 & Glla & fig. $25-9$ & lignes horizontales au peigne à dents ovales & locus 2-UF 16 - 78 et 200 \\
\hline 21 & Bla & fig. $21-17$ & cordon préoral et poinçon & locus 2-UF $16-81$ \\
\hline 22 & FIlb & fig. $20-10$ & croisillons au peigne & locus 2-UF 16 - 74 \\
\hline 23 & Fllb & fig. $25-18$ & échelles au peigne & locus 2-UF 24 - 249 \\
\hline 30 & Glla & fig. $30-12$ & échelles au peigne & locus 6-UF $41-116$ \\
\hline 31 & $\mathrm{H}$ & fig. $30-8$ & échelles au peigne & locus 6-UF 41 - 297 \\
\hline 32 & Gla & fig. $30-19$ & languette & locus 6-UF $41-128$ et 306 \\
\hline 33 & Glla & fig. 13-13 & anse en boudin & décap. méc.-UF 42 - 283 \\
\hline 34 & BII & fig. 13-6 & pied de coupe polypode & diagnostic \\
\hline 35 & Bla & fig. 21-6 & anse en boudin & locus 2-UF $16-105$ \\
\hline 36 & A & fig. 22-5 & fermeture Éclair au peigne et au poinçon & locus 2-UF 27 - 15 \\
\hline 37 & Flla & fig. 14-6 & lignes horizontales au peigne & locus 1-UF 02 - 200 \\
\hline 38 & GIII & fig. $25-4$ & échelles et chevrons au peigne & locus 2-UF 23 - 56 et UF 24 - 247 \\
\hline 39 & Gllb & fig. 15-1 & cordon préoral & locus 1-UF 17 - 314 \\
\hline 40 & Glla & fig. 17-18 & cordon préoral & locus 1-UF 19 - 269 \\
\hline 41 & $\mathrm{FI}$ & fig. 17-20 & cordon préoral & locus 1 -UF 19 - 268 \\
\hline 42 & Glb & fig. 23-2 & cordon préoral & locus 2-UF 29 - 44 \\
\hline 43 & Glla & fig. 19-18 & cannelure & locus 1-UF $22-1$ \\
\hline 44 & Glla & fig. $22-16$ & coupe non décorée & locus 2-UF 27 - 26 \\
\hline 45 & EII & fig. 15-11 & gobelet non décoré & locus 1-UF $03-224$ \\
\hline
\end{tabular}

figure permet d'affirmer que non seulement des vases décorés (ou leur argile constitutive) ont voyagé, mais également la céramique domestique (ou les argiles constitutives).

Enfin, pour finir avec la première famille, les vases uniques de chaque groupe $\mathrm{C}$ et $\mathrm{D}$ sont décorés et corres- pondent respectivement à un gobelet et à une coupe hémisphérique à fond plat.

Toutes proportions gardées, l'éventail des formes est relativement diversifié au sein de cet ensemble de vases fabriqués à partir des argiles les plus lointaines. 
Dans la seconde famille, le groupe E ne contient que deux vases communs; l'un est une jarre à cordon préoral $\left(n^{\circ} 3\right)$ et l'autre un gobelet non décoré $\left(n^{\circ} 45\right)$. Le groupe $F$, fort de neuf individus, renferme indifféremment des vases décorés et communs. Les deux vases du sous-groupe I correspondent à une jarre trouvée enterrée $\left(\mathrm{n}^{\circ} 1\right)$ et à une coupe à cordon préoral ( $\left.n^{\circ} 41\right)$. Le sous-groupe II mêle des céramiques communes, un vase à cordon préoral $\left(\mathrm{n}^{\circ} 8\right)$ et une faisselle ( $\left.n^{\circ} 28\right)$, à des gobelets décorés $\left(n^{\circ *} 17,22,23\right.$, 27 et 37$)$.

Le groupe $G$ renferme plus de la moitié des vases analysés (24 individus). Comme les autres, il a fait l'objet de partitions internes et, hormis pour le sous-groupe III, chaque ensemble individualisé regroupe un minimum de trois individus pouvant aller jusqu'à huit. À l'échelle du groupe, la proportion entre la céramique commune et les vases décorés est paritaire. Le sous-groupe I subdivisé en a et b contient cinq vases décorés et quatre communs. En Ia, les gobelets décorés ( $n^{* \$} 4,9,14$ et 25 ) voisinent avec un vase en forme de tonnelet à cannelure sous le bord $\left(n^{\circ} 7\right)$ et avec un autre récipient de morphologie inconnue, mais portant une languette ( $\left.n^{\circ} 32\right)$. En Ib, un seul gobelet décoré ( $\left.n^{\circ} 16\right)$, un vase à cordon préoral portant des coups d'ongle ( $n^{\circ} 29$ ) et une coupe hémisphérique ( $\left.\mathrm{n}^{\circ} 42\right)$, sont présents. Le sousgroupe II est fort de quatorze céramiques. Il a été subdivisé en deux sous-groupes a et $\mathrm{b}$ renfermant respectivement huit et six individus. Trois gobelets décorés ( $\mathrm{n}^{\text {os }} 20,26$ et 30 ), deux vases à cordon préoral ( $n^{\text {os }} 40$ et 43 ), une coupe ( $\left.n^{\circ} 44\right)$, un bord de gobelet non décoré $\left(n^{\circ} 2\right)$ et une anse en boudin ( $\left.n^{\circ} 33\right)$ sont classés en IIa. Trois gobelets décorés $\left(n^{\circ} 5,15\right.$ et 19), deux récipients à cordon préoral $\left(n^{\% s} 11\right.$ et 39 ) et une anse en boudin ( $\left.n^{\circ} 18\right)$ sont rangés en IIb. De fortes similitudes pétrographiques ont été notées dans la pâte de tous les vases de IIa. Les rapprochements sont les suivants: $n^{\text {os }} 30,40$ et 43 ainsi que 2 et 33 ou encore 20,26 et 44 . L'ensemble 30,40 et 43 correspond à un gobelet décoré $\left(n^{\circ} 30\right)$ et à deux céramiques communes à cordon préoral ( $n^{\text {os }} 40$ et 43 ). Le gobelet décoré $\left(n^{\circ} 5\right)$ de IIb est également très proche de cet ensemble. Les individus $n^{\circ s} 20$ et 26 correspondent à des gobelets décorés portant le même type de décor tandis que le vase $n^{\circ} 44$ est une céramique commune. En revanche, les vases $n^{\text {os }} 2$ et 33 sont tous deux des récipients communs. Le vase $n^{\circ} 39$ de IIb leur est également proche.

Dans deux cas, les similitudes de pâtes concernent à la fois des vases décorés et des céramiques communes. Ces exemples illustrent bien le fait qu'une même terre sert indif- féremment à fabriquer aussi bien les céramiques fines décorées que les récipients domestiques plus grossiers.

Enfin, le seul individu du sous-groupe GIII ( $n^{\circ} 38$ ) est un gobelet décoré ainsi que le vase unique du groupe $\mathrm{H}$ (n'31).

Tout comme la première famille, chaque groupe et sousgroupe est constitué d'un ensemble de céramiques à la fois décorées et domestiques de morphologie variée. Les gobelets décorés aussi bien que les récipients non décorés ont été fabriqués à partir de terres semblables. Un même type morphologique a pu être confectionné avec des types de terres différents. Par rapport à la première famille, les localisations des niveaux utilisés se rapprochent du site, mais les modes d'exploitation restent identiques à ceux qui ont été décrits précédemment.

\section{Avec la technologie des décors et les thèmes décoratifs}

Au sein de la céramique commune, l'ensemble des vases à cordons préoraux modelés est pétrographiquement hétérogène. En effet, ces récipients sont classés dans les groupes B, E, F et G, c'est-à-dire dans les ensembles numériquement les plus fournis. Dans les groupes $F$ et $G$, ils se répartissent également dans les deux sous-groupes I et II. Par conséquent, les argiles employées pour la fabrication de ces vases à cordon sont nettement différentes, ce qui implique des origines géographiques distinctes. Rien ne peut être déduit des autres types de céramiques communes qui sont uniques.

Les gobelets décorés d'impressions réalisées avec un peigne sont au nombre de vingt-et-un. Parmi eux, le groupe des vases à lignes horizontales représente neuf individus. Hormis le vase $n^{\circ} 10$ classé dans le groupe $D$ de la première famille, les autres céramiques proviennent de différents sous-groupes de E, F et G. Les décors à base d'échelles se trouvent en $\mathrm{H}, \mathrm{F}$ et $\mathrm{G}$, dans cinq sousgroupes différents. Les hachures, les triangles et les croisillons ont été imprimés sur des gobelets fabriqués à partir d'argiles variées, essentiellement de la seconde famille. Le vase décoré de lignes horizontales incisées ( $\left.n^{\circ} 14\right)$ appartient au sous-groupe GIa. Plus intéressant, le décor de "fermeture Éclair » a été imprimé sur le seul vase du groupe $A$, fabriqué avec une terre assurément éloignée du site.

\section{Étude des inclusions d'origine anthropique}

Seule la présence de chamotte a été notée. Rappelons qu'il s'agit de débris de céramiques qui ont été réduites 
en poudre et incorporées dans l'argile au moment de la préparation de la pâte. Les grains possèdent les caractéristiques de céramiques et peuvent donc être étudiés de la même manière. Vingt-six céramiques en renferment,

Tabl. XVIII - Corrélation des vases chamottés avec les groupes pétrographiques.

\begin{tabular}{|c|c|c|}
\hline Pétro. & N $^{\circ}$ Éch. & Chamotte \\
\hline A & 36 & - \\
\hline Bla & 21 & - \\
\hline Bla & 35 & - \\
\hline Blb & 13 & - \\
\hline Bll & 34 & - \\
\hline Bllla & 24 & - \\
\hline Blllb ou Ell & 12 & + \\
\hline C & 6 & - \\
\hline D & 10 & - \\
\hline El & 3 & +++ \\
\hline Ell & 45 & ++ \\
\hline Fl & 1 & - \\
\hline Fl & 41 & + \\
\hline Flla & 17 & +++ \\
\hline Flla & 28 & + \\
\hline Flla & 37 & +++ \\
\hline Fllb & 8 & ++ \\
\hline Fllb & 22 & +++ \\
\hline Fllb & 23 & +++ \\
\hline Fllb & 27 & + \\
\hline Gla & 4 & +++ \\
\hline Gla & 7 & + \\
\hline Gla & 9 & + \\
\hline Gla & 14 & - \\
\hline Gla & 25 & - \\
\hline Gla & 32 & - \\
\hline Glb & 16 & - \\
\hline Glb & 29 & + \\
\hline Glb & 42 & ++ \\
\hline Glla & 2 & - \\
\hline Glla & 20 & + \\
\hline Glla & 26 & +++ \\
\hline Glla & 30 & - \\
\hline Glla & 33 & + \\
\hline Glla & 40 & +++ \\
\hline Glla & 43 & ++ \\
\hline Glla & 44 & + \\
\hline Gllb & 5 & - \\
\hline Gllb & 11 & +++ \\
\hline Gllb & 15 & + \\
\hline Gllb & 18 & +++ \\
\hline Gllb & 19 & ++ \\
\hline Glll & 39 & - \\
\hline
\end{tabular}

soit un peu plus de la moitié du corpus analysé. Signalons également qu'un seul grain était présent dans le champ d'observation de la lame mince du vase $n^{\circ} 30$. L'abondance des grains est variable, de quelques

Tabl. XIX - Répartition des vases chamottés par locus et par UF.

\begin{tabular}{|c|c|c|}
\hline Répartition spatiale & $\mathbf{N}^{\circ}$ Éch. & Chamotte \\
\hline locus 1-UF 01 - 315 & 9 & + \\
\hline locus 1-UF $01-316$ & 5 & - \\
\hline locus 1-UF $01-317$ & 8 & ++ \\
\hline locus 1-UF 01 - 318 & 6 & - \\
\hline locus 1-UF 01 - 319 & 7 & + \\
\hline locus 1-UF $15-161$ & 18 & +++ \\
\hline locus 1-UF 17 - 314 & 39 & - \\
\hline locus 1-UF 18 - 276 & 16 & - \\
\hline locus 1-UF 18 - 282 & 13 & - \\
\hline locus 1 -UF $19-260$ & 12 & + \\
\hline locus 1-UF 19 - 264 et 267 & 11 & +++ \\
\hline locus $1-$ UF $19-268$ & 41 & + \\
\hline locus 1-UF 19 - 269 & 40 & +++ \\
\hline locus 1-UF 19 - 34 & 17 & + \\
\hline locus 1-UF 02 - 199 & 10 & - \\
\hline locus 1-UF 02 - 200 & 37 & +++ \\
\hline locus 1-UF 22 - 230 & 19 & ++ \\
\hline locus 1-UF $03-217$ & 15 & + \\
\hline locus 1-UF $03-222$ & 14 & - \\
\hline locus 1-UF 03 - 224 & 45 & ++ \\
\hline locus 2-UF 13 - 207 & 4 & +++ \\
\hline locus 2-UF 13 - 205 & 2 & - \\
\hline locus 2-UF 13 - 213 & 3 & +++ \\
\hline locus 2-UF 16 - 105 & 35 & - \\
\hline locus 2-UF 16 - 202 & 24 & - \\
\hline locus 2-UF $16-73$ et UF $23-55$ & 26 & +++ \\
\hline locus 2-UF 16 - 74 & 22 & +++ \\
\hline locus 2-UF 16 - 76 et 71 et 75 & 27 & + \\
\hline locus 2 -UF 16 - 78 et 200 & 20 & + \\
\hline locus 2-UF $16-81$ & 21 & - \\
\hline locus 1 -UF 22 - 1 & 43 & ++ \\
\hline locus 2-UF 23 - 56 et UF 24 - 247 & 38 & - \\
\hline locus 2-UF 24 - 249 & 23 & +++ \\
\hline locus 2-UF 27 - 15 & 36 & - \\
\hline locus 2-UF 27 - 26 & 44 & + \\
\hline locus 2-UF 27 - 33 & 25 & - \\
\hline locus 2-UF $29-44$ & 42 & ++ \\
\hline locus 4-UF $33-139$ et UF $34-148$ & 28 & + \\
\hline locus 4-UF 34 - 152 & 29 & + \\
\hline locus 5-UF 09 - 311 & 1 & - \\
\hline locus 6-UF $41-116$ & 30 & - \\
\hline locus 6-UF 41 - 128 et 306 & 32 & - \\
\hline locus 6-UF 41 - 297 & 31 & - \\
\hline décap. méc.-UF 42 - 283 & 33 & + \\
\hline diagnostic & 34 & - \\
\hline
\end{tabular}


individus à plusieurs dizaines pour une même surface et ne semble pas être en rapport avec la densité initiale en particules. En effet, une quantité importante de grains de chamotte a parfois été ajoutée dans des argiles déjà

Tabl. XX - Corrélation de la chamotte avec les données typotechnologiques.

\begin{tabular}{|c|c|c|}
\hline Type de vase - Technique et thèmes décoratifs & No Éch. & Chamotte \\
\hline jarre & 1 & - \\
\hline coupe non décorée & 44 & + \\
\hline gobelet non décoré & 45 & ++ \\
\hline bord de gobelet non décoré & 2 & - \\
\hline cordon préoral & 11 & +++ \\
\hline cordon préoral & 39 & - \\
\hline cordon préoral & 40 & +++ \\
\hline cordon préoral & 12 & + \\
\hline cordon préoral & 3 & +++ \\
\hline cordon préoral & 41 & + \\
\hline cordon préoral & 43 & ++ \\
\hline cordon préoral & 42 & ++ \\
\hline cordon préoral & 8 & ++ \\
\hline cordon préoral et coups d'ongle & 29 & + \\
\hline cordon préoral et poinçon & 21 & - \\
\hline lignes horizontales à la spatule & 13 & - \\
\hline vase tonnelet à cannelure sous le bord & 7 & + \\
\hline languette & 32 & - \\
\hline faisselle & 28 & + \\
\hline anse en boudin à tenon & 18 & +++ \\
\hline anse en boudin & 33 & + \\
\hline anse en boudin & 35 & - \\
\hline pied de coupe polypode & 34 & - \\
\hline bandes hachurées au peigne & 4 & +++ \\
\hline bandes hachurées au peigne & 6 & - \\
\hline lignes horizontales au peigne & 26 & +++ \\
\hline lignes horizontales au peigne & 16 & - \\
\hline lignes horizontales au peigne & 37 & +++ \\
\hline lignes horizontales au peigne & 9 & + \\
\hline lignes horizontales au peigne & 19 & ++ \\
\hline lignes horizontales au peigne & 5 & - \\
\hline lignes horizontales au peigne & 27 & + \\
\hline lignes horizontales au peigne à dents ovales & 20 & + \\
\hline bandes de lignes horizontales et triangles rayés au peigne & 10 & - \\
\hline triangles rayés au peigne & 17 & + \\
\hline croisillons au peigne & 15 & + \\
\hline croisillons au peigne & 22 & +++ \\
\hline hachures et chevrons au peigne & 24 & - \\
\hline échelles et métopes au peigne & 25 & - \\
\hline échelles au peigne & 23 & +++ \\
\hline échelles au peigne & 30 & - \\
\hline échelles au peigne & 31 & - \\
\hline échelles et chevrons au peigne & 38 & - \\
\hline fermeture Éclair au peigne et au poinçon & 36 & - \\
\hline lignes horizontales incisées & 14 & - \\
\hline
\end{tabular}

riches en inclusions naturelles. La taille des grains oscille le plus souvent entre $150 \mu \mathrm{m}$ et $2 \mathrm{~mm}$, mais peut parfois atteindre $4 \mathrm{~mm}$.

Le croisement des données issues des études sur l'origine des argiles utilisées pour fabriquer la céramique (tabl. XVIII) et de la présence/absence de la chamotte montre quelques tendances, particulièrement affirmées pour les céramiques de la première famille. En effet dans cette famille, seul le vase $\mathrm{n}^{\circ} 12$ renferme des grains de chamotte. Or, il a été vu supra que cet individu, pauvre en inclusions naturelles, n'a peut-être pas été classé dans la bonne famille. Il serait donc à ranger en EII plutôt qu'en BIIIb. Il semble donc que la pratique d'introduction de chamotte dans la pâte soit plutôt à mettre au crédit des personnes qui ont fabriqué les céramiques de la seconde famille. Ce résultat est important car il se calque sur la coupure majeure réalisée au cours de l'étude pétrographique. Hormis un individu $\left(\mathrm{n}^{\circ} 1\right)$, les vases du groupe $\mathrm{F}$ sont tous chamottés. Le groupe $G$ apparaît comme étant plus complexe. En effet, les vases à chamotte sont majoritairement présents dans les différents sous-groupes, mais jamais exclusifs. Le sous-groupe GIIa renferme des séries de vases à pâte similaire qui présentent différents cas de figure. Dans la première série, les vases $n^{\text {os }} 40$ et 43 renferment de la chamotte tandis que le $\mathrm{n}^{\circ} 30 \mathrm{n}$ 'en contient pas. Dans la deuxième série, les trois vases $\mathrm{n}^{\text {os }} 20,26$ et 44 sont chamottés. Enfin, dans la dernière série ( ${ }^{\text {os }} 2$ et 33 ), seul l'individu $\mathrm{n}^{\circ} 33$ en renferme.

Enfin, le seul vase du groupe $\mathrm{H}$ ne contient aucun grain. La répartition des vases à chamotte au sein des locus (tabl. XIX) ne montre aucune relation particulière. Ils sont indifféremment présents sur toute la surface fouillée sauf, peut-être, pour le locus 6 , mais sur seulement trois individus. Une même structure (locus 1, UF 1 ou UF 2) renferme à la fois des vases avec et sans chamotte.

La pâte des céramiques communes aussi bien que celle des vases décorés renferme de la chamotte (tabl. XX). Néanmoins, son emploi semble être plus important dans les céramiques communes. En effet, $65 \%$ d'entre elles en renferment, contre $50 \%$ pour la céramique décorée. Les vases à cordon préoral s'individualisent au sein de l'ensemble des céramiques communes car neuf récipients, sur les onze vases à cordon préoral (soit $82 \%$ ), renferment de la chamotte.

La présence de chamotte ne semble pas liée à une catégorie de module volumétrique puisque plusieurs contenances sont représentées. Elle ne montre également 
aucun lien avec une fonction particulière de la céramique culinaire ou de stockage par exemple.

En ce qui concerne la céramique décorée, largement dominée par les gobelets, quelques tendances se dessinent. En effet, la pâte de six gobelets sur neuf, portant des lignes horizontales réalisées au peigne, et la pâte d'un seul vase décoré d'échelles ( $\mathrm{n}^{\circ}$ 23) sur les cinq renferment des grains de chamotte. Celle du gobelet à décor de "fermeture Éclair " n'en contient pas, tout comme le vase portant des lignes horizontales incisées. Les vases décorés de bandes hachurées ou croisillonnées réalisées au peigne sont trop rares pour que la présence ou l'absence de chamotte soit significative.

L'observation fine de la composition des grains de chamotte apporte également des renseignements sur la génération antérieure de pots. En effet, comme les grains sont le résultat du broyage de vases brisés puis incorporés dans l'argile, ce sont donc les restes d'anciens vases présents sur le lieu de production au moment de la préparation de la pâte qui sont réduits en poudre. Au moins une céramique fabriquée avec une argile de la seconde famille renferme des éléments de vases de la première famille. Le seul critère qui peut être véritablement pris en compte correspond à la nature aciculaire de la matrice, seulement observée pour l'unique individu rangé dans le groupe A. En effet, la pâte du vase $n^{\circ} 45$ classé en EII, donc à mica noir abondant, renferme quelques grains de chamotte dont la matrice a un aspect aciculaire et présente également une forte proportion de micas noirs, caractéristique des vases du groupe $\mathrm{E}$ et surtout du groupe B. D'autres vases renferment des grains appartenant à d'anciennes céramiques classées dans les groupes fortement chargés en mica noir. Les deux autres vases chamottés dont la pâte est riche en biotite ( $\mathrm{n}^{\text {os }} 3$ et 12) renferment aussi des grains abondants en mica noir. Les autres céramiques dont une partie des grains de chamotte est riche en mica noir $\left(\mathrm{n}^{\mathrm{os}} 4,8,9,11,15,18,28,29,37,40\right.$, 42 et 43) possèdent elles-mêmes des matrices avec un taux en biotite beaucoup plus bas. Ces vases sont classés dans FIIa et $b$, GIa et b et GIIa et b, c'est-à-dire dans la plupart des sous-groupes de la deuxième famille (fig. 42). Mais la majorité des vases, y compris les individus $\mathrm{n}^{\text {os }} 3$ et 45 , renferment de la chamotte du type "matrice des groupes $F$ et $G$ ", certaines d'entre elles possédant même des éléments calcédonieux ( $\mathrm{n}^{\mathrm{os}} 18,23$ et 26 ).

Sur chaque lieu de fabrication des céramiques de la seconde famille existent des récipients mis au rebut puis broyés, présentant une gamme de composition pétrographique extrêmement variable.
Aucun grain proche de la matrice du seul individu du groupe $\mathrm{H}$ n'a été détecté.

La présence de chamotte contenue dans les grains euxmêmes permet de reculer encore d'une génération les possibilités d'analyse. Ainsi, les pâtes de trois générations de céramiques peuvent être connues. Trois vases présentent cette possibilité ( $\mathrm{n}^{\mathrm{os}} 28,42$ et 45$)$, classés respectivement en FIIa, GIb et EII. Les grains de la première génération des vases $\mathrm{n}^{\text {os }} 28$ et 42 sont identiques à ceux de la chamotte de la deuxième génération. En revanche, la seule particule de première génération du récipient $\mathrm{n}^{0} 45$ est différente. Présente dans une matrice aciculaire riche en micas noirs, elle montre une matrice phylliteuse renfermant du quartz. Une céramique fabriquée dans un contexte "type groupe A " peut donc renfermer de la chamotte, ce qui n'est pas le cas pour l'unique représentant analysé sur le site.

L'étude de la chamotte complétant la première analyse pétrographique montre un enchevêtrement de cas de figure extrêmement difficile à démêler qui est la conséquence de l'exploitation d'innombrables sources argileuses au cours d'une période relativement brève.

\section{Discussion et éléments de conclusion}

La mise en évidence de deux grandes familles de terres géologiquement et géographiquement distinctes est primordiale et implique un certain nombre de déductions. Tout d'abord, la totalité des ressources se trouve en rive droite de la Saône alors que La Noue se situe en rive gauche. Les matières premières ou les céramiques déjà fabriquées ont donc dû obligatoirement traverser la rivière avant d'arriver sur le site.

Les argiles de la seconde famille potentiellement les plus proches de La Noue, et en particulier les terres du groupe $G$ les plus abondantes, ont pu être directement exploitées par les occupants du site. En revanche, le cas des argiles de la première famille, localisées à l'ouest, sur la Côte chalonnaise, à une distance majoritairement supérieure à $20 \mathrm{~km}$, mérite une discussion. En effet, une exploitation directe de terres aussi éloignées du site est peu conforme aux situations ethnoarchéologiques observées. Les travaux de D. Arnold (1985) en Amérique centrale et du Sud ont mis en évidence la présence d'un seuil situé à $7 \mathrm{~km}$ du lieu de confection de la céramique. $86 \%$ des sources argileuses proviennent d'une distance inférieure à ce seuil. Les $14 \%$ qui restent donnent des argiles qui sont acheminées par des moyens modernes non disponibles dans le passé. Dans une 
étude similaire réalisée en Afrique subsaharienne, O. Gosselain (2002) obtient un seuil de $3 \mathrm{~km}$, bien inférieur à celui de D. Arnold, pour $95 \%$ des sources. Pourquoi aller chercher des terres à plus de $30 \mathrm{~km}$ pour certaines d'entre. elles alors que des ressources locales sont disponibles et ont été abondamment exploitées? De plus, à cette famille de terres lointaines semble correspondre l'absence (ou quasiabsence) de chamotte dans la pâte des neuf individus, confirmant la coupure observée dans l'analyse pétrographique, cette particule étant essentiellement un marqueur de la seconde famille. Néanmoins, l'étude de la composition des grains de chamotte nuance ce résultat car elle a fait apparaître que de la céramique de " type groupe A " pouvait être chamottée.

Dans le cas oì toute la réramique serait fabriquée à partir de ressources argileuses collectées depuis La Noue, peut-être à l'occasion de déplacements liés à d'autres activités, la production serait donc autarcique. En revanche, si la présence de ces céramiques est le résultat, sur le site, de l'introduction de récipients et non d'argiles et que chaque nappe de vestiges, assimilée à une unité d'habitation à occupation durable, renferme à la fois des vases locaux et ces récipients étrangers, comment peut-on expliquer cette situation? Traduit-elle des relations entre des occupants pérennes ou temporaires de basse plaine et des populations vivant dans des établissements d'altitude, ou bien seulement la présence d'occupants temporaires installés tantôt à La Noue, tantôt dans les monts du Charollais, dans le cadre de déplacements saisonniers? Dans le premier cas, les vases les plus lointains correspondraient à des produits étrangers au groupe de La Noue acquis par chaque maisonnée, tandis que dans le second cas, ce serait une production propre à ces gens. En effet, dans ce dernier cas, les vases de la première famille pourraient correspondre seulement aux récipients nécessaires dans les premiers temps de leur installation. Ensuite, les occupants de La Noue fabriqueraient leur poterie à partir des argiles locales appartenant à la seconde famille.

Dans l'hypothèse d'une occupation saisonnière, il serait curieux que le même groupe humain n'introduise pas (ou peu) de chamotte dans des vases fabriqués en altitude avant d'être emportés à La Noue et, qu'ensuite, en introduise dans la pâte de ceux qui sont fabriqués sur place. La faiblesse numérique de la première famille est peut-être à l'origine d'un biais dans la série étudiée et la seule analyse pétrographique ne permet pas à elle seule de trancher quant à la nature de la production de ces vases fabriqués à partir d'argiles lointaines. Néanmoins, en dépit de ces restrictions, l'hypothèse d'une arrivée de vases plutôt que d'argiles sera privilégiée, les distances apparaissant comme importantes pour une collecte directe dans le cadre d'une. économie domestique.

Le répertoire morphologique est varié dans les deux familles de terres. Dans les deux cas, les céramiques correspondent à la fois à des vases décorés et à des récipients domestiques. Il n'existe pas non plus de relation privilégiée entre un morphotype et une terre. Les types de production restent également identiques pour les deux familles. Au niveau de la technique décorative et des thèmes, aucun lien n'a été mis en évidence avec la nature de la terre. Tout type d'argile a été employé pour la fabrication à la fois de vases communs et décorés avec des techniques et des thèmes variés.

En revanche, l'introduction de chamotte semble être plus importante pour la céramique commune que pour les vases décorés majoritairement au peigne. Si cette tendance était confirmée, cela signifierait que, dès la préparation de l'argile, le potier sait à quelle fonction va être dévolue sa production. De plus au sein des vases, aussi bien domestiques que décorés, les morphotypes ou les répertoires décoratifs semblent plus ou moins liés avec l'emploi de chamotte.

F. C.

\section{COMPOSANTES CULTURELLES DES INDUSTRIES SILICEUSES TAILLÉES}

Si les principales composantes du Campaniforme sont identifiées à partir de la typologie céramique (Gallay, 1986; Besse, 1996a; Salanova, 1996), les industries lithiques taillées n'en constituent pas moins une source documentaire complémentaire et essentielle à la caractérisation de la culture matérielle du Campaniforme domestique (Fouéré, 1994 ; Detrey, 1997, 2002; Bailly, 2002b). Issue d'un contexte d'habitat à stratification unique et composée d'ensembles clos, la série siliceuse taillée de La Noue représente une production industrielle homogène et suffisamment conséquente ( $n=301$ ), pour alimenter la connaissance du fonctionnement économique du Campaniforme régional. Une fois décrite et caractérisée, des comparaisons à plus longues distances, pourront être établies avec d'autres industries taillées de référence localisées dans l'hexagone, en particulier au nord et à l'est de la France, et dans la partie occidentale de la Suisse (Billard et al., 1991; Bailly, 2002b; 
Detrey, 2002 et 2003). L'approche spatiale sera réalisée à partir de la distribution des artefacts taillés par locus et en fonction des aires de concentration de mobilier.

Les éléments taillés recueillis correspondent à un échantillonnage dont la représentativité pourrait être mise en balance avec celle d'un corpus de silex plus représentatif, qui engloberait au moins deux maisonnées complètes d'un hameau.

Seuls deux produits taillés en chaille dont un éclat Levallois et un éclat pseudo-Levallois à retouches latérales ont été identifiés. Il s'agit là néanmoins des seuls silex anachroniques et résiduels parmi l'industrie campaniforme exhumée à La Noue, qui n'ont pas été dessinés et englobés dans le décompte total de cette série, dans le cadre de cette étude. Le matériel lithique non siliceux, représenté par 5 pierres de quartzite ou de grès, a été traité séparément car bon nombre d'entre ces éléments ne sont pas manufacturés.

Le corpus exhumé de La Noue compte 301 silex, les esquilles étant incluses à ce total (tabl. XXI). Globalement, la série lithique se subdivise en deux catégories d'artefacts: l'une rassemble les produits de débitage, les nucléus et les pièces techniques $(n=238)$; l'autre regroupe l'ensemble des produits façonnés et modifiés par retouches ainsi que les produits bruts utilisés ( $n=63$ ) (fig. 44).

Le préalable de cette d'étude a consisté à s'interroger sur les choix des artisans, depuis l'acquisition des matériaux

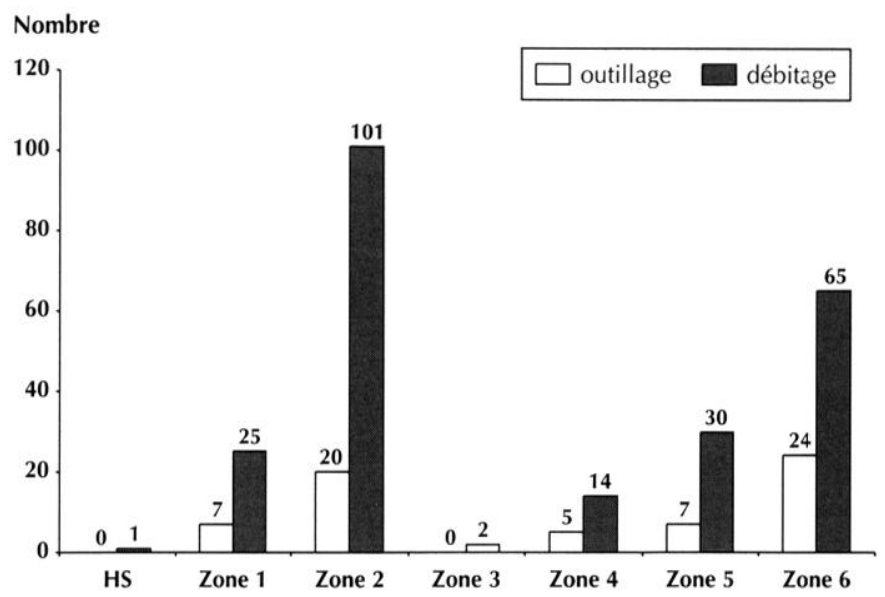

Fig. 44 - Répartition des outils et des produits de débitage de La Noue.

jusqu'à la réalisation de l'outil, sur l'identification des processus de débitage à partir des modules des supports et sur les intentions des tailleurs en vue d'obtenir des produits standardisés ou non. L'approche environnementale, la gestion des matières dans le cadre du territoire exploité et au sein du site lui-même ont valeur égale pour caractériser l'économie de l'industrie taillée. Ainsi, la mise en évidence des savoir-faire techniques et des choix culturels, influant ou non sur la sélection des outillages, permet d'appréhender un des assemblages matériels du groupe campaniforme de La Noue.

Tabl. XXI - Décompte de la série lithique.

\begin{tabular}{|l|c|c|c|c|c|c|c|c|}
\hline Type d'industrie & HS & Zone 1 & Zone 2 & Zone 3 & Zone 4 & Zone 5 & Zone 6 & Totaux \\
\hline Grattoir & & 2 & 12 & & 4 & 3 & 11 & $\mathbf{3 2}$ \\
\hline Pièce esquillée & & 1 & 3 & & & 1 & 4 & $\mathbf{9}$ \\
\hline Éclat à ret. lat. & & & 2 & & & 1 & 4 & $\mathbf{7}$ \\
\hline Lamelle à ret. lat. & & 1 & & & & & 3 & $\mathbf{4}$ \\
\hline Encoche & & 2 & 1 & & & & 1 & $\mathbf{4}$ \\
\hline Retouchoir & & & & & & 1 & & $\mathbf{1}$ \\
\hline Denticulé & & 1 & & & & 1 & & $\mathbf{2}$ \\
\hline Perçoir & & & & & & & 1 & $\mathbf{1}$ \\
\hline Pointe de flèche & & & 1 & & 1 & & & $\mathbf{2}$ \\
\hline Lame de poignard & & & 1 & & & & & $\mathbf{1}$ \\
\hline Éclat utilisé ? & & 10 & 21 & & 2 & 6 & & $\mathbf{3 9}$ \\
\hline Éclat & & 5 & 35 & & 6 & 17 & 9 & $\mathbf{7 2}$ \\
\hline Lamelle & & 3 & 4 & & 1 & 2 & 2 & $\mathbf{1 2}$ \\
\hline Casson & & 5 & 6 & & 4 & & 17 & $\mathbf{3 2}$ \\
\hline Esquille & & & 16 & 1 & 1 & 4 & 20 & $\mathbf{4 2}$ \\
\hline Nucléus & 1 & 1 & 8 & & & 1 & 4 & $\mathbf{1 5}$ \\
\hline Pièce technique & & 1 & 10 & 1 & & & 13 & $\mathbf{2 5}$ \\
\hline Percuteur & & & 1 & & & & & $\mathbf{1}$ \\
\hline Totaux & $\mathbf{1}$ & $\mathbf{3 2}$ & $\mathbf{1 2 1}$ & $\mathbf{2}$ & $\mathbf{1 9}$ & $\mathbf{3 7}$ & $\mathbf{8 9}$ & $\mathbf{3 0 1}$ \\
\hline
\end{tabular}




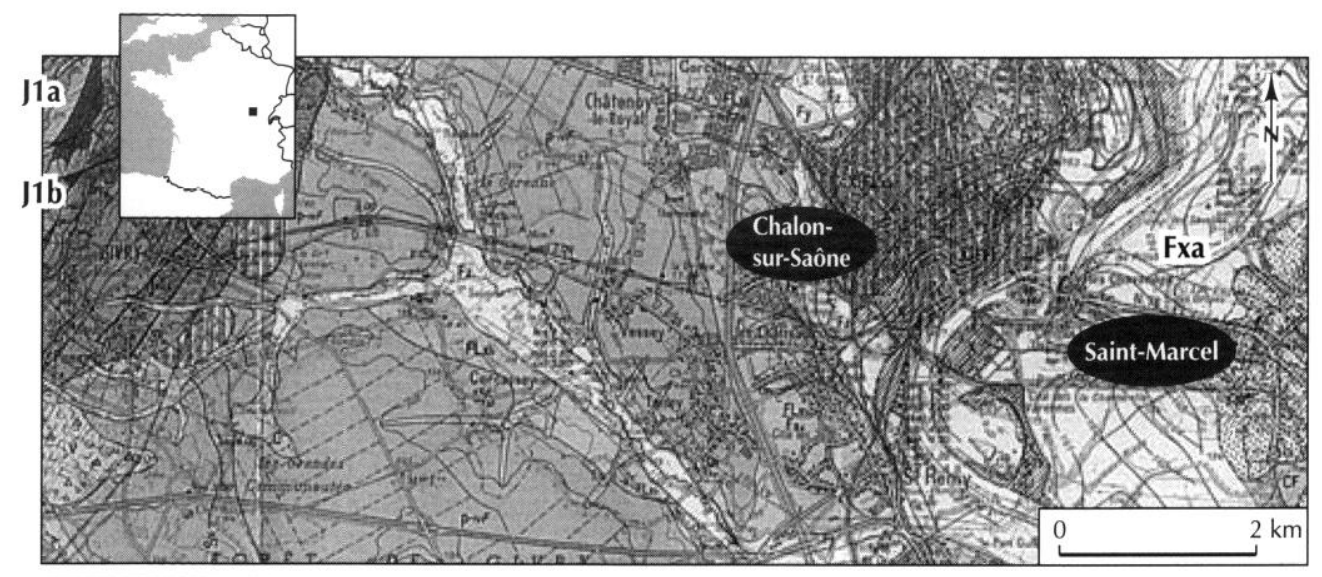

J1a Bajocien supérieur : calcaires à silex

J1b Bajocien inférieur : nodules et petits rognons de silex

Fig. 45 - Localisation des sources potentielles de silex (d'après carte BRGM, $n^{0} 579$ ).

\section{MATIÈRES PREMIÈRES}

L'approche sur les matières premières a été réalisée à partir de l'observation de 236 silex (soit $87,4 \%$ du total) car 38 d'entre eux sont brûlés (soit 12,6\% du total) et donc rendus illisibles par les cupules et les fissurations qui affectent les surfaces des supports.

Le potentiel en roches siliceuses est faiblement représenté si l'on se réfère, entre autres, à la carte géologique BRGM locale (Bonvalot et al., 1984a, 1984b). Parmi les grandes formations affleurantes, deux possibilités d'acquisition directe en ressources siliceuses s'offrent, d'une part, sur le site lui-même, et d'autre part, le long des contreforts de la Côte châlonnaise, en rive droite de la Saône (fig. 45). Les premières formations sont celles du Quaternaire, Fy et Fz, qui sont des formations alluviales récentes formées de sables et de graviers, où des éléments siliceux roulés sont présents mais pas suffisamment exploitables pour façonner des industries; en revanche en Fxa, sur la rive droite de la Saône, des cordons de petits galets siliceux apparaissent localement. Il s'agirait là de bancs de silex du Crétacé, démantelés et repris dans des alluvions. Les deuxièmes formations sont celles du Secondaire, situées à une dizaine de kilomètres à l'ouest du site, où des nodules et des petits rognons de silex sont présents dans l'étage du Bajocien inférieur et moyen (J1a et $\mathrm{J} 1 \mathrm{~b}$ ). Le distinguo entre ces matières n'est pas aisé à réaliser car l'aspect général des roches est identique à cœur, c'est-à-dire, à grain fin de couleur beige caramel, parfois doté d'une légère patine blanche. Les cortex sont généralement fins à peu épais et vacuolés, certains d'entre eux sont roulés et étoilés, confirmant un glanage effectué directement par les occupants de La Noue dans les formations alluviales proches du site. Les modules de ces galets ou de ces rognons roulés n'excèdent pas 8 à $10 \mathrm{~cm}$. Un galet de chaille testé et utilisé comme percuteur est le seul exemplaire de ce type parmi la série. Comme le silex, la chaille qui est une roche peu siliceuse a été récupérée sur place par les occupants de La Noue.

La matière première majoritairement employée est donc essentiellement locale, voire sub-locale, n'excédant pas une journée de marche. Les silex du Secondaire, affleurant dans les contreforts du Châlonnais, sont accessibles à partir du franchissement de gués, le long du cours de la Saône. Aucune source d'approvisionnement en silex exogène n'a été identifiée, prouvant ainsi une certaine « autonomie " des Campaniformes de La Noue, dans ce cas, exclusivement en rapport avec l'approvisionnement en roches siliceuses. Nous nous interrogerons plus tard sur cette relative indépendance des tailleurs, en fonction du choix des matières exploitées, à mettre ou non en parallèle avec les options techniques et typologiques identifiées.

\section{MÉTHOdeS ET TECHNiQUeS DE TAILle}

Tous locus confondus, le débitage est majoritairement représenté par rapport aux produits manufacturés et utilisés, puisqu'il correspond à $79 \%$ du total de la série lithique (fig. 46 à 52). Sur une base de 238 produits de débitage décomptés, 37 silex sont brûlés. L'approche des 
différents modes opératoires de taille prend en compte les nucléus, les éclats de première et de seconde entame, les pièces dites " techniques ", regroupant les flancs de nucléus, les tablettes de ravivages de plan de frappe ainsi que les lames à crête. Nombre d'entre ces éléments caractéristiques sont répartis dans chacun des six locus distingués lors de la fouille, mais ils seront traités en même temps afin d'appréhender globalement les chaînes opératoires.

\section{Produire des supports avec nucléus}

Deux types de production de support ont été distingués, l'un avec nucléus, et l'autre sans nucléus. Le premier schéma est le plus employé car 16 nucléus entiers ont été recensés et se distinguent comme des modèles informes, multipolaires globuleux, unipolaires pyramidaux ou prismatiques, polyédriques et discoïdes.

\section{Les nucléus informes}

Ceux-ci sont au nombre de 2 individus et ne présentent pas une morphologie définissable car ils sont utilisés jusqu'à exhaustion du support et, peut-être, réemployés comme outils (percuteur, pierre de lestage...).

\section{Les nucléus multipolaires globuleux}

Quatre nucléus globuleux, à enlèvements multipolaires ont été décomptés. Trois d'entre eux issus du locus 2, de même facture, sont vraisemblablement produits par le même artisan tailleur. Ces modèles se retrouvent en très faible proportion parmi les industries campaniformes, comme à Alle et Chevenez (Detrey, 2002), ou à Géovreissiat (Bailly, 1997-1998, 2002b). Un petit exemplaire comporte des esquillements alternes et opposés, ce qui induirait son réemploi après abandon, le support ayant été exploité jusqu'à exhaustion (fig. 48, nº 10).

\section{Les nucléus unipolaires pyramidaux}

Ces modèles, au nombre de 3 exemplaires (fig. 47, n"11; fig. 52, $n^{\circ} 7$ ), montrent un plan de frappe principal à partir duquel partent des enlèvements lamellaires convergents vers la base du nucléus (Billard et al., 1991 ; Bailly, 19971998, 2002b). Certains d'entre ces types montrent une plage corticale résiduelle (fig. 52, n" 7). Un seul nucléus unipolaire sub-pyramidal à lamelles, chauffé préalablement à la taille, fait partie du corpus; cependant aucune lamelle débitée à partir de cet exemplaire n'a été identifiée.

\section{Les nucléus unipolaires courts à lamelles}

Deux nucléus à plan de frappe préférentiel ont été décomptés parmi la série de La Noue. Ces exemplaires à enlèvements d'éclats unidirectionnels et à enlèvements lamellaires sub-convergents ne sont pas de même facture et ne semblent pas être produits par la même main. L'un de ces deux nucléus, sur galet cortical de petit module, comporte un plan de frappe réaménagé par une série d'enlèvements convergents. En cours de réfection, cet exemplaire a néanmoins été abandonné en l'état (fig. 46, n*8).

\section{Les nucléus prismatiques à éclats}

Trois nucléus prismatiques, dont l'un est à plans croisés, sont des modèles dérivés des nucléus pyramidaux; le plan de frappe étant plus réduit et étroit pour les modèles de type prismatiques que pour les modèles pyramidaux (Billard et al., 1991) (fig. 52, $\mathrm{n}^{\text {os }} 6$ et 8 ).

\section{Le nucléus polyédrique à éclats}

Alors que ce modèle est fréquent parmi les séries campaniformes (Fouéré, 1994; Detrey, 1997; Bailly, 1997-1998, 2002b), un seul nucléus de ce type a été recensé. Cet exemplaire, exhumé dans le locus 2 , possède quatre plans de frappe et il adopte une morphologie cubique ayant pour dimension $3 \mathrm{~cm}$ de côté environ.

\section{Le nucléus discoïde à éclats}

Un exemplaire unique de ce type a été identifié (fig. 48, $\mathrm{n}^{\circ} 8$ ). Il présente un débitage circulaire intentionnel à partir d'éclats centripètes. Sur la face inférieure du nucléus, un enlèvement esquillé et envahissant en partie distale résulte d'un débitage sur enclume, obtenu à la percussion directe. Ce mode opératoire, qui relève d'un concept levalloisien, aléatoire, semble-t-il dans ce cas, se rapproche de celui qui a été utilisé sur trois galets à Alle, Noir Bois. Il s'agit dans les deux cas observés d'un schéma opératoire de même principe et, a priori, non prédéterminé (Detrey, 1997).

\section{Les fragments de nucléus et le percuteur sur galet}

Correspondant à ces éléments de débitage, 16 fragments de nucléus, difficilement identifiables, ont été décomptés. Un galet de chaille testé comme un nucléus bipolaire potentiel a été réemployé comme percuteur (fig. $49, \mathbf{n}^{\circ} 11$ ). Il semblerait, dans ce cas, que l'abandon de ce nucléus sur galet ait été guidé par un choix significatif (d'ordre culturel?), et non suivant une simple option technique, car la chaille se taille et s'emploie comme le silex, à l'exception 

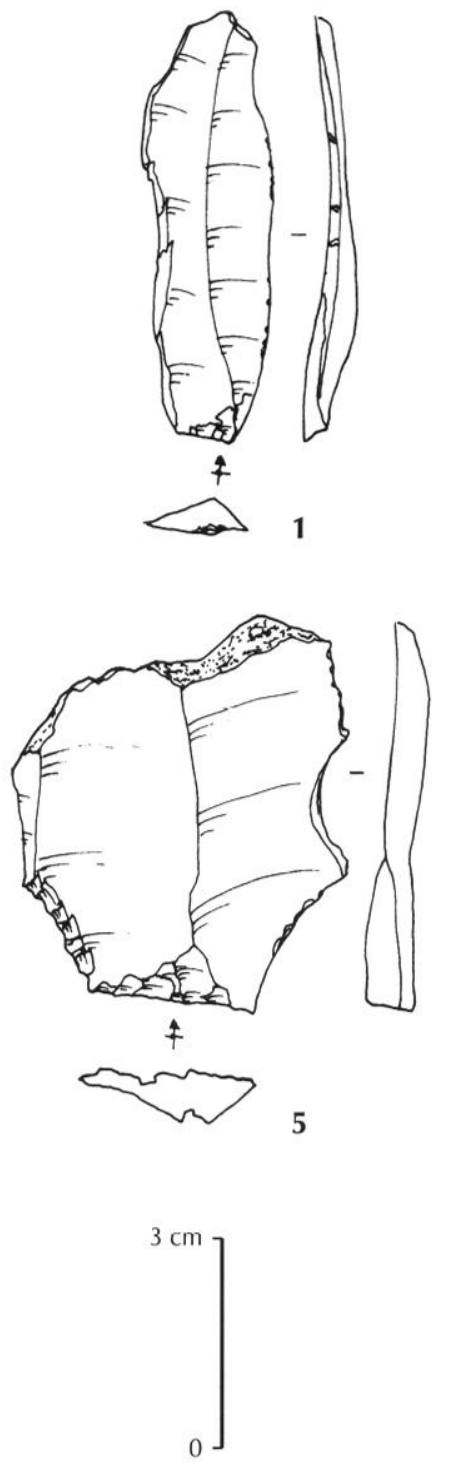

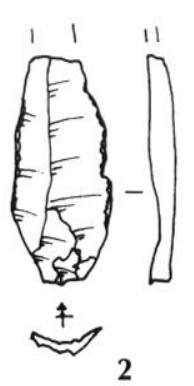

2

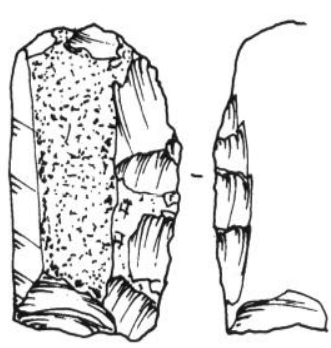

6

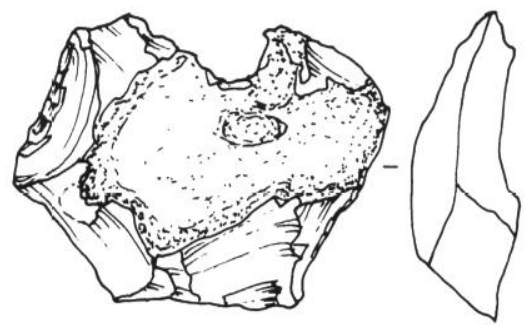

9
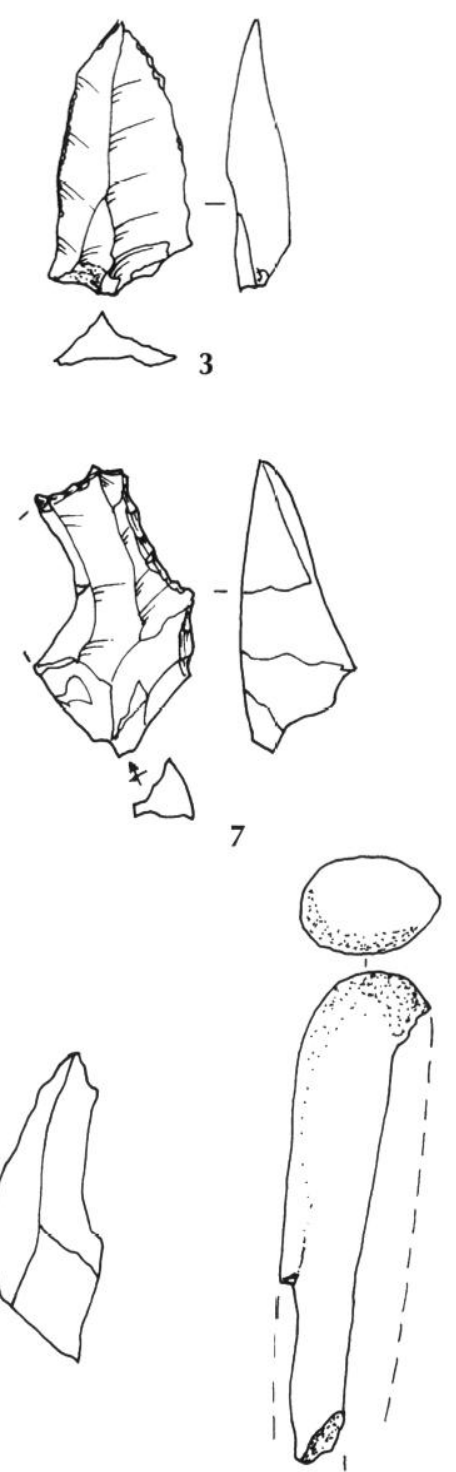

10
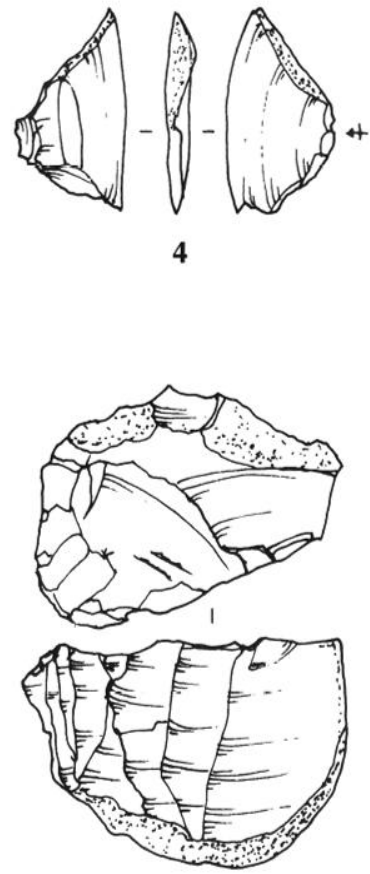

8

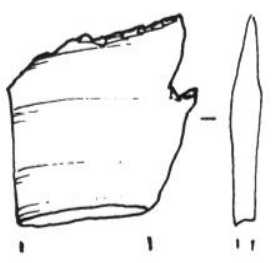

11

Fig. 46 - 1-11 : produits de débitage du locus 1 (dessin S. Saintot).

près que ce premier matériau est moins siliceux que le second. Pour preuve: un seul éclat de chaille a été recensé parmi la totalité de l'industrie recueillie à La Noue, les deux autres produits fabriqués à partir de cette roche étant datés du Paléolithique moyen (voir p. 96).

Le débitage obtenu à partir de chaines opératoires avec nucléus est dominé par des éclats $(n=118)$, mais également par la présence non négligeable des lames courtes et des lamelles $(n=16)$. Les supports sont obtenus suivant un mode unidirectionnel et rarement croisé ou orthogonal. De nombreux flancs de nucléus confirment le réaménagement des nucléus pour en extraire le maximum de supports. De plus, ces plans de frappe sont réemployés comme supportsoutils pour le façonnage de grattoirs ou celui de pièces esquillées. Une lamelle antéro-latérale avec pyramidion constitue l'unique pièce technique attestant un mode de préparation de nucléus à lamelle (fig. $48, \mathrm{n}^{\circ} 4$ ). Dans le locus 6 , quatre lamelles sont produites par pression (fig. 52 , $\mathrm{n}^{\text {os }} 1$ à 4 ), en particulier le $\mathbf{n}^{\circ} 4$, si l'on tient compte de la rectitude des nervures et de la présence du talon ponctiforme. Parmi les produits de débitage recueillis dans ce même locus, une lamelle à crête, et une lamelle avec pyramidion pourraient également faire partie d'une chaîne 

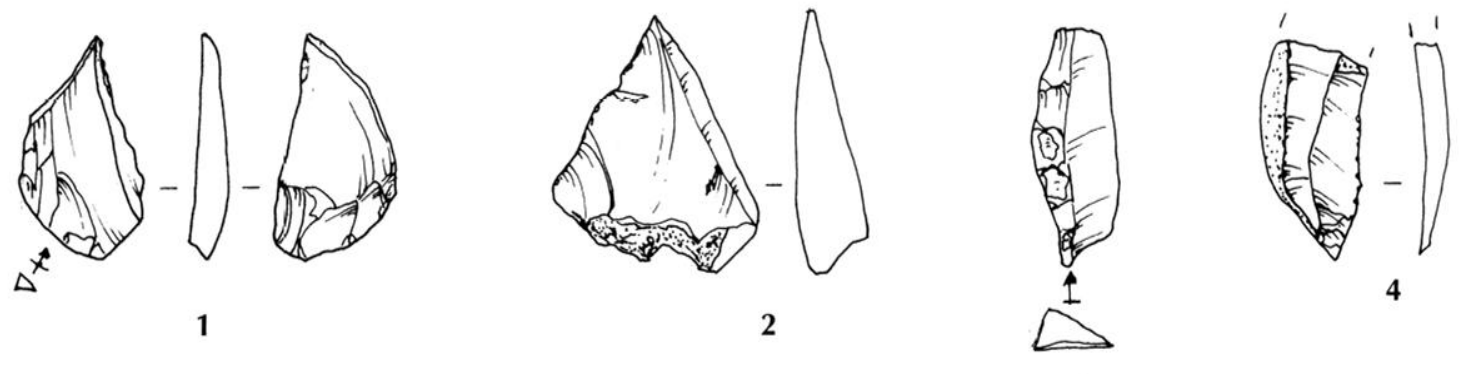

एक्षिए

3
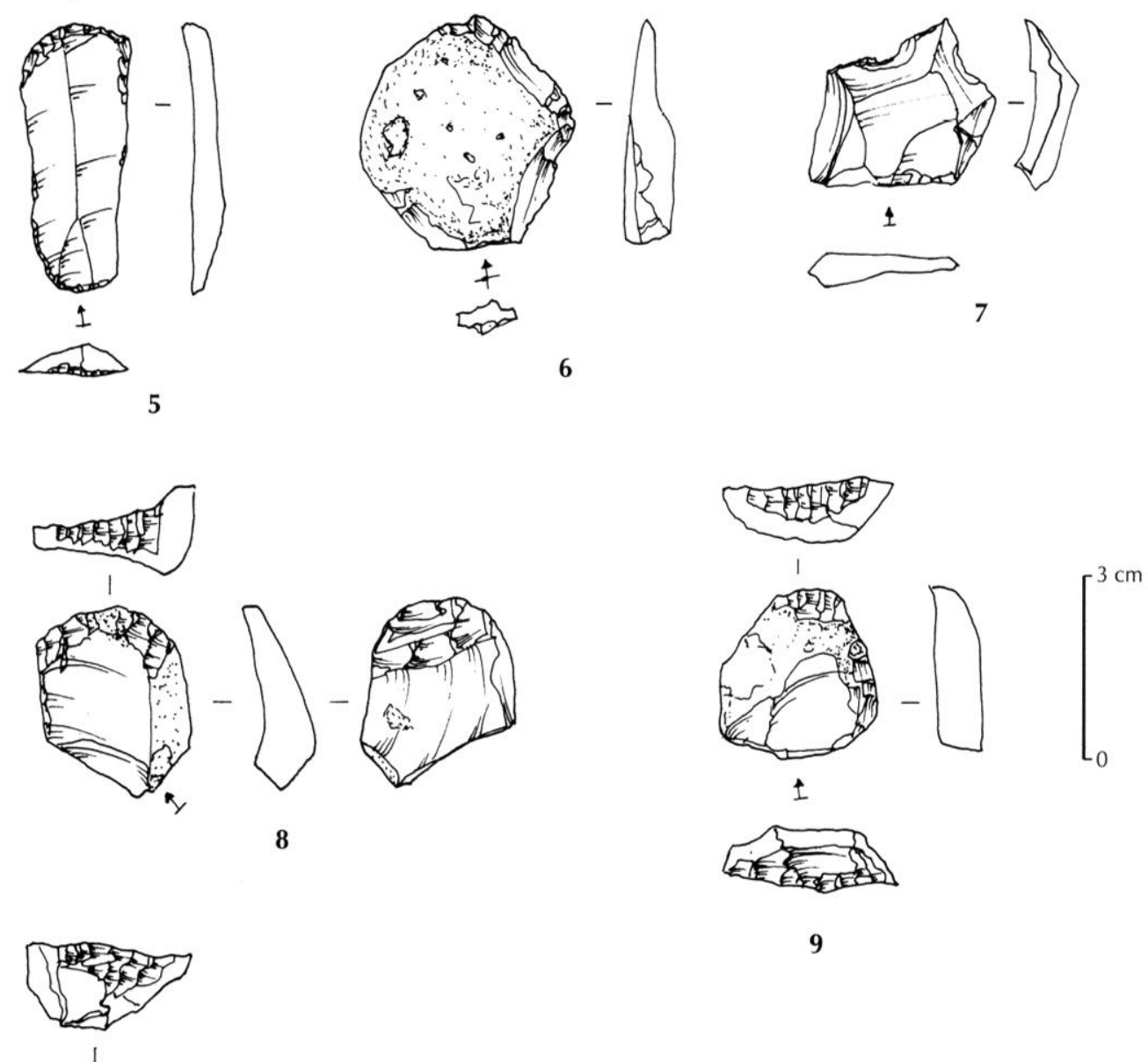

9

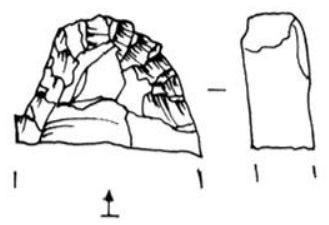

10
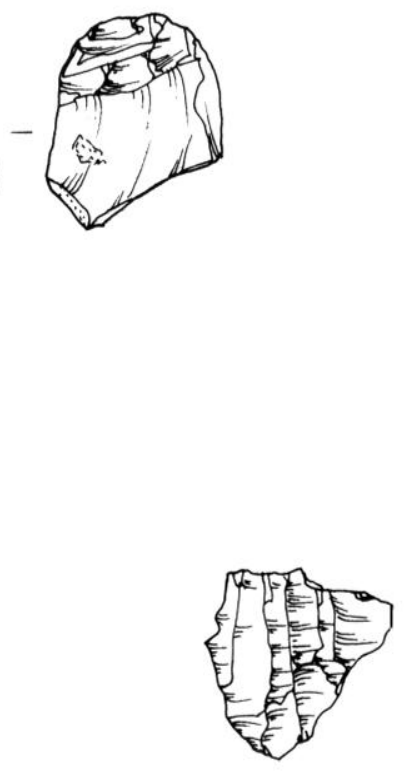

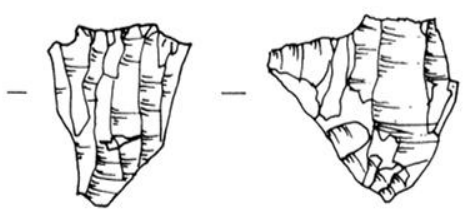

11

Fig. $47-1-11$ : outils du locus 1 (dessin S. Saintot). 

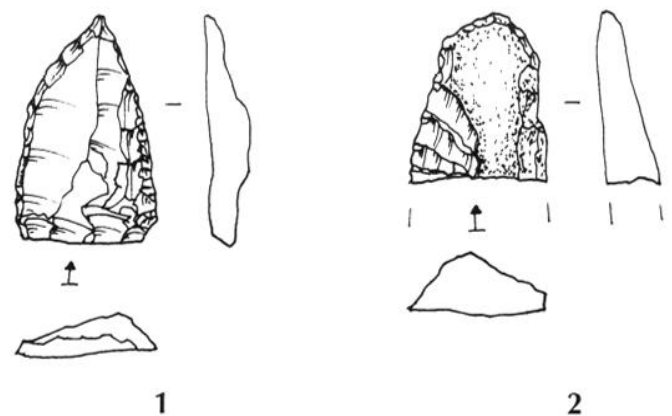

2

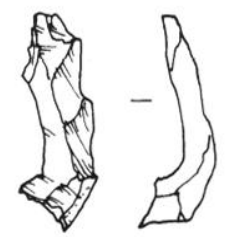

4

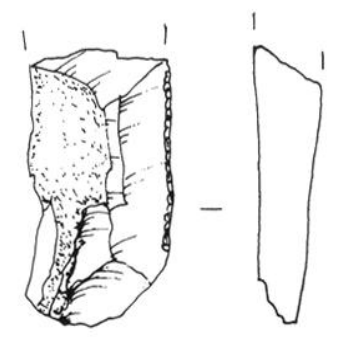

$x$

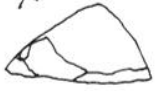

7
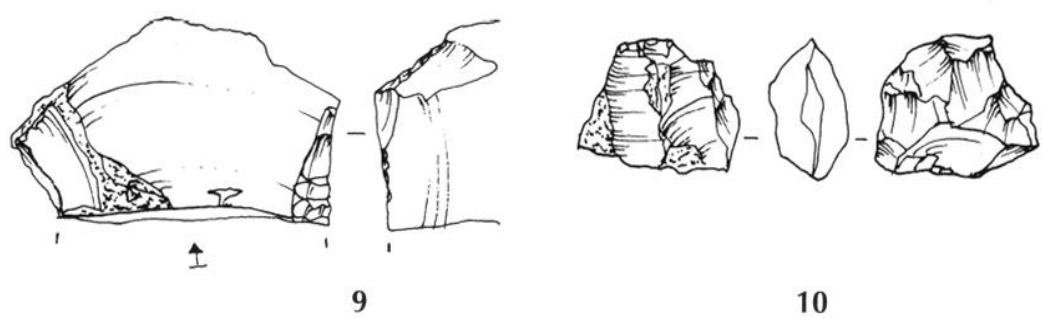

10

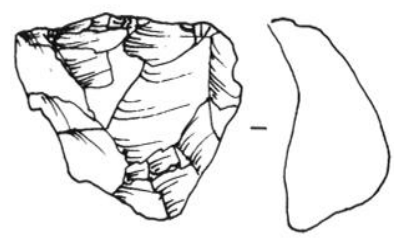

11

Fig. 48 - 1-11 : outils et produits de débitage du locus 2 (dessin S. Saintot). 


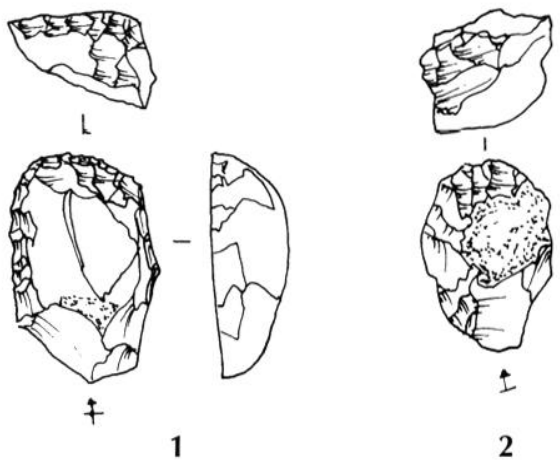

(1)

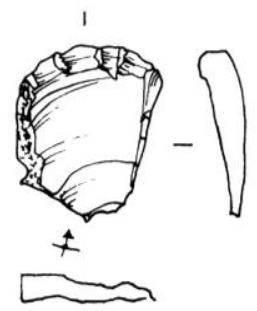

3
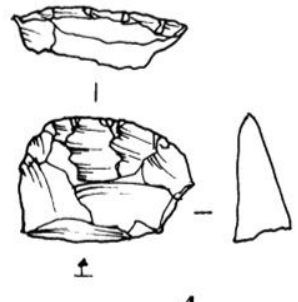

4
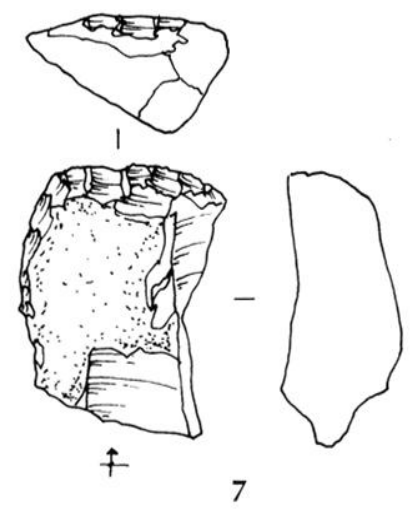

₹ 5
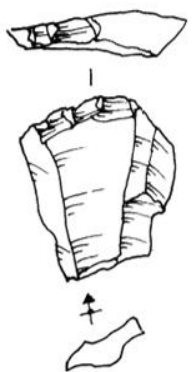

8

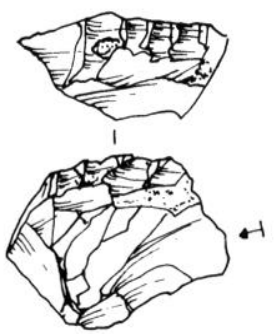

9
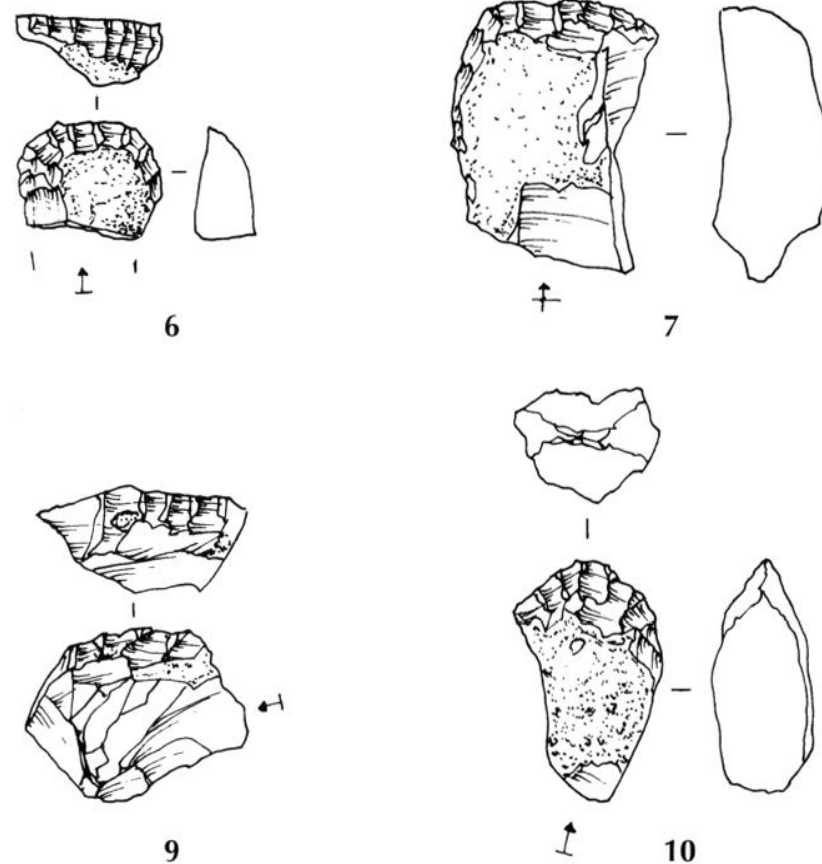

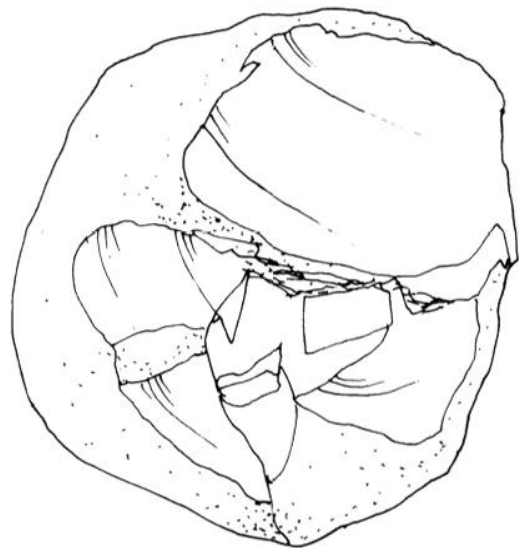

11

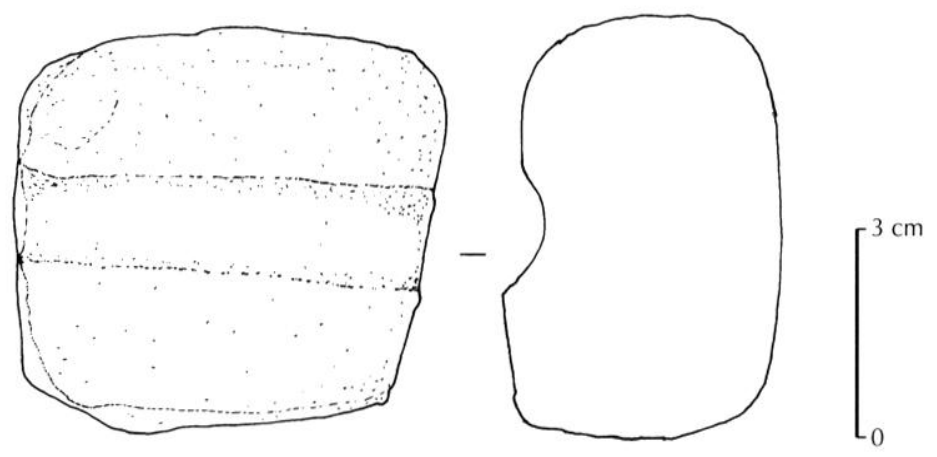

12

Fig. 49 - 1-12: grattoirs du locus 2 (dessin S. Saintot). 
opératoire utilisée par un spécialiste pour produire ce type de support lamellaire. Dans le locus 1, quatre lames courtes sont taillées à la percussion indirecte (fig. $46, \mathrm{n}^{\circ} 1$ à 3), ce qui correspond à un mode de débitage occasionnel. Ine. lamelle bitronquée à trois pans peut également avoir été produite à la percussion indirecte (fig. 50, $\mathrm{n}^{\circ} 4$ ), mais il s'agit là d'un élément isolé parmi la série. Malgré la présence de lames courtes, de fines lamelles à trois pans et de nucléus pyramidaux évoquant un débitage semblable à une production mésolithique, aucun microlithe ou élément de type Montbani n'a été identifié comme aux Grandes Corvées à Vandières, Meurthe-et-Moselle (Boura, 1993).

\section{Produire des supports sans nucléus}

Ce mode opératoire est plus difficile à déceler car il laisse une faible proportion de chutes de taille caractéristiques et parce que, dans le cas des industries de La Noue, aucun remontage direct n'a pu être réalisé. En dépit de ces facteurs restrictifs, du point de vue de l'interprétation des chaînes opératoires et de la détermination des produits, vingt silex correspondant à des éclats corticaux, d'entames et à des supports aménagés et retouchés ou utilisés appartiennent à ce processus de production.

Il s'agit d'un débitage de petit galet par percussion sur enclume, opérant suivant deux modes: le premier procédé consiste à scinder en deux un galet et à aménager les deux moitiés séparément, le second mode revient à débiter un galet ou un petit rognon par tranches. L'absence de nucléus caractérise ces deux schémas opératoires. Les stigmates de taille lisibles sur les supports produits sur enclume présentent des esquillements sur les faces inférieures et comportent une tranche corticale partielle ou totale. Parmi les éléments les plus représentatifs, signalons:

- 1 éclat produit sur tranche de galet (fig. 50, n”3), provenant du locus 4 ;

- 6 silex, dont 3 éclats produits sur galet en " quartier d'orange " présentent un fil tranchant brut, sont peut-être employés comme tels (fig. $46, \mathrm{n}^{\circ} 4$; fig. 47 , nº 1 ; fig. $51, \mathrm{n}^{\circ} 2$ ) et trois éclats esquillés, dont les talons sont corticaux (fig. $47, \mathrm{n}^{\text {os }} 2,8$ et 9 );

- et 2 produits esquillés débités sur enclume, issus du locus 6 (fig. 52, $\mathrm{n}^{\text {os }} 9$ et 10 ).

Dans le locus 2, deux séries d'éclats à tranche corticale, en " quartier d'orange " sont vraisemblablement produites à partir de deux galets débités sur enclume à la percussion dure directe, et ceci à défaut de remontage. Ces lacunes parmi les chaînes opératoires pourraient s'expliquer par l'emploi de certains produits à l'extérieur du site. La gestion de la matière première sous-tend une production immédiate de supports bruts, utilisables sous cette forme ou aménagés sommairement. Dans ce cas, l'investissement technique requis est donc moindre par rapport au mode de production avec nucléus.

Ce mode de gestion et d'obtention des supports a été employé sur d'autres séries campaniformes parmi lesquelles nous retiendrons la série d'Alle, Noir Bois (Detrey, 1997), celle des Florentins à Val-de-Reuil (Billard et al., 1991), ainsi que la série de Maupas à Calvisson (Fouéré, Roger, 2002).

\section{GeSTION DES DÉBITAGES ET DES MATIÈRES PREMIÈRES}

Sur la base de l'identification des modes d'acquisition des matières premières et sur celle de l'évaluation des différentes chaînes opératoires, force est de constater une production dominée par les éclats se place dans la classe des produits courts, compris entre $2 \mathrm{~cm} \leq \mathrm{L} \leq 4 \mathrm{~cm}$ (fig. 53). Les produits lamellaires et laminaires courts ne dépassant pas, ou rarement, les $5 \mathrm{~cm}$ (fig. $46, \mathrm{n}^{\circ} 1$ ). Les talons lisibles sont majoritairement plans $(60 \%)$, corticaux ou non corticaux, préparés $(15 \%)$, linéaires $(20 \%)$, ou ponctiformes $(5 \%)$.

Le débitage est donc principalement produit à la percussion directe au percuteur dur $(90 \%)$, occasionnellement à la percussion indirecte $(7 \%)$ et exceptionnellement à la pression (3\%). Le débitage sur enclume est attesté, ce qui a déjà été observé (Detrey, 1997; Bailly, 2002b; Fillion, 2002), néanmoins aucune pierre à cupule et aucun bloc-enclume n'ont été recueillis sur le site, sur la surface fouillée.

L'outillage inclut tous les produits, aménagés ou non, mais retouchés, même de façon occasionnelle, suite à l'utilisation du support. Dans ce dernier cas, il s'agit d'outils a posteriori. Un premier classement typologique a été proposé, à partir duquel une catégorie, celle des éclats retouchés, a été individualisée. Celle-ci regroupe plusieurs produits bruts $(n=39)$, dont l'un des bords ou le fil tranchant latéral ou distal ont peut-être été utilisés, ce qui ne pourrait être confirmé ou infirmé qu'à partir d'une analyse tracéologique.

Les supports-outils sélectionnés pour le façonnage des outillages sont morphologiquement éclectiques, corticaux (50\%) et de petits modules, ne dépassant pas, ou rarement, les $4,5 \mathrm{~cm}$ de long pour la catégorie des grattoirs (fig. 54). Pour conclure sur ce sujet, l'outillage de La Noue est globalement peu diversifié et peu investi techniquement, l'objectif principal des artisans-tailleurs de ce site consistant à fabriquer rapidement quelques outils pour les utiliser dans l'immédiat. 

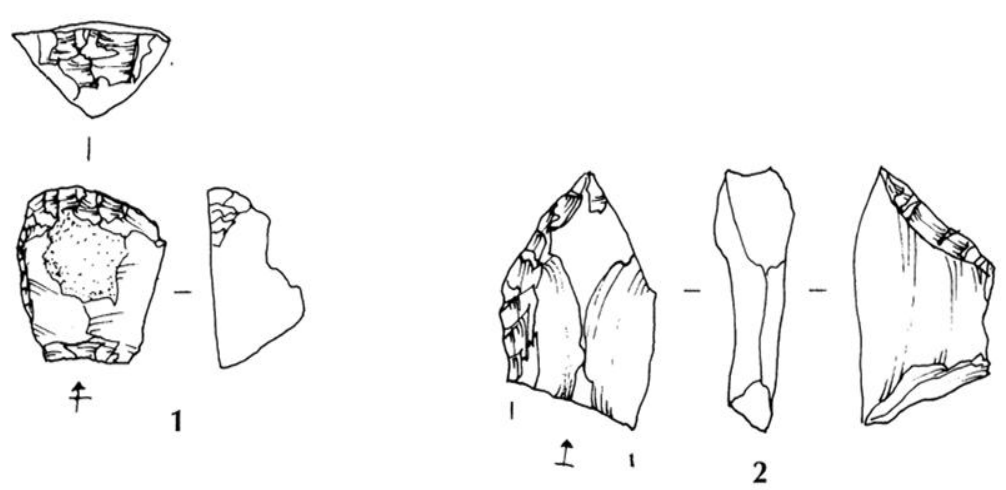

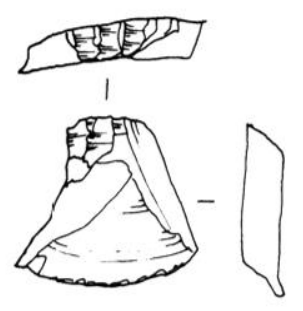

3

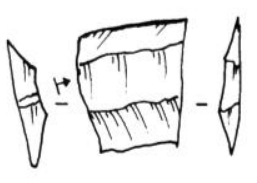

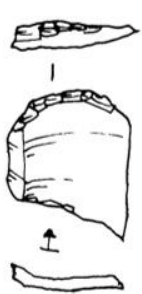

5

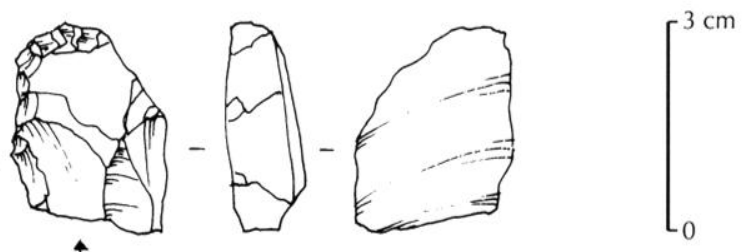

千

6

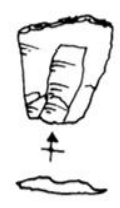

7

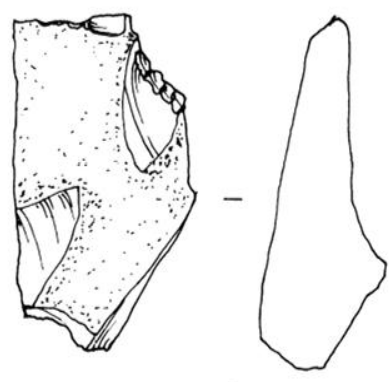

8

Fig. 50 - 1-8: industrie lithique des locus 3, 4 et 5 (dessin S. Saintot). 

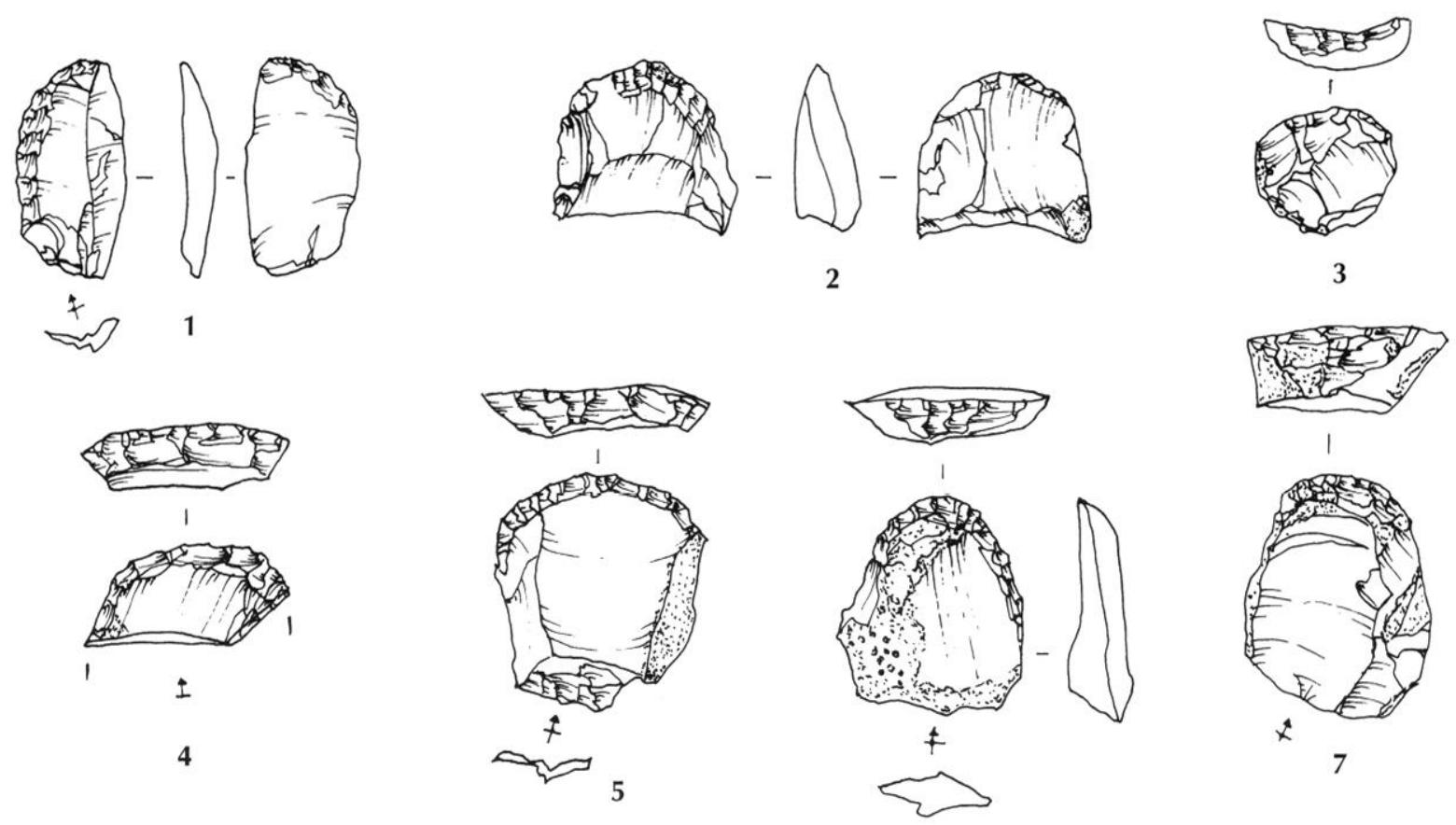

4

6
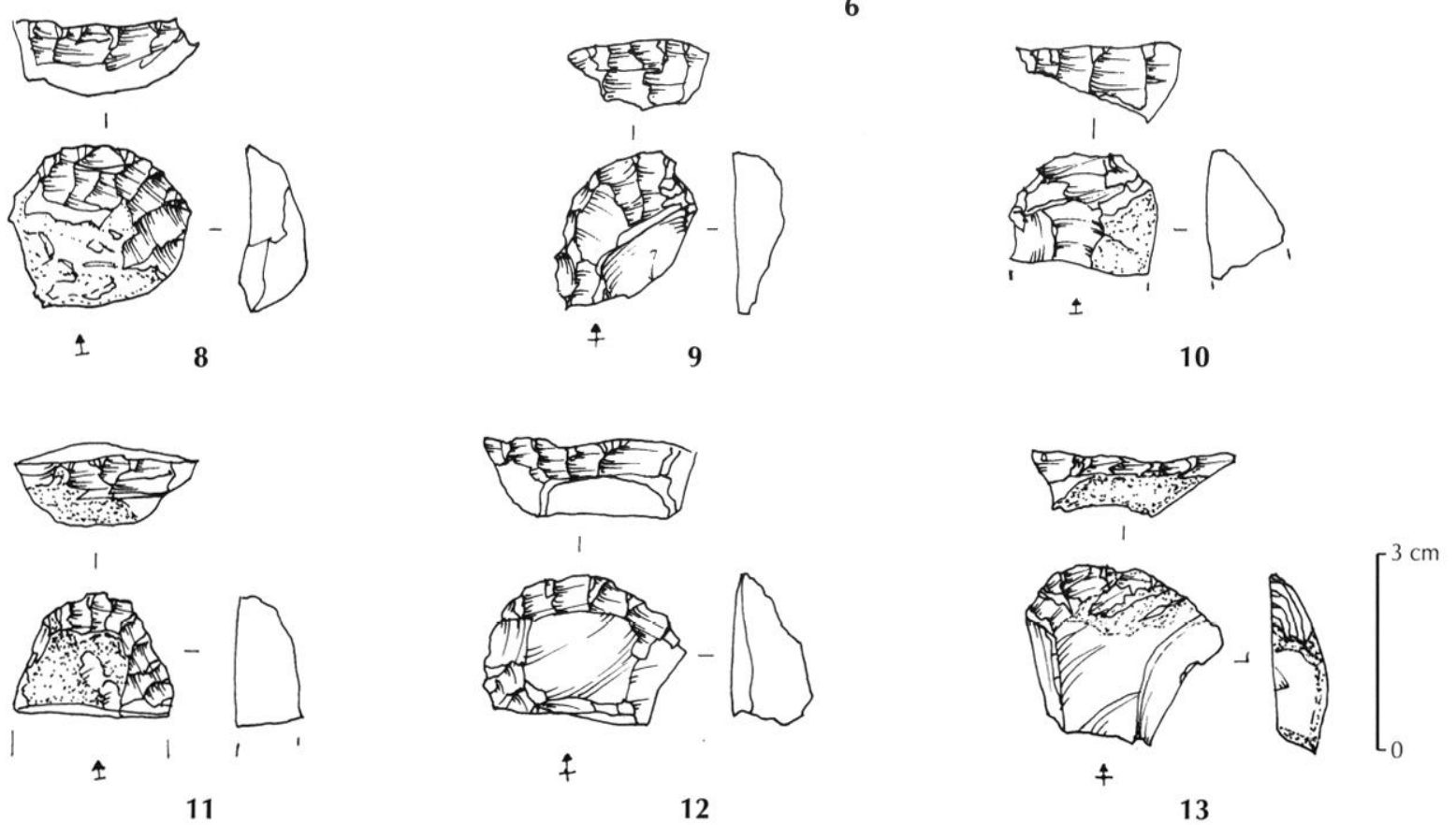

Fig. $51-1-13$ : outils du locus 6 (dessin S. Saintot). 


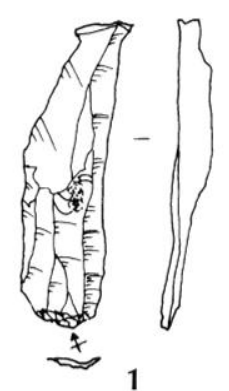

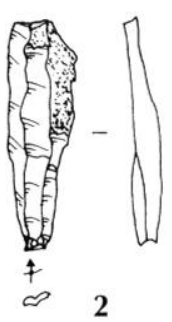
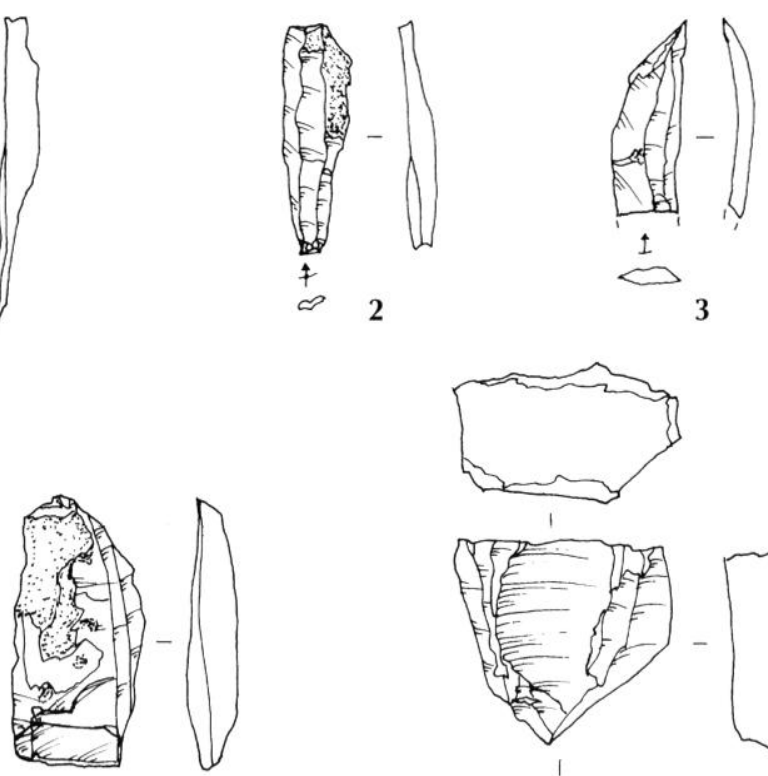

$\uparrow$

$\nsim 5$

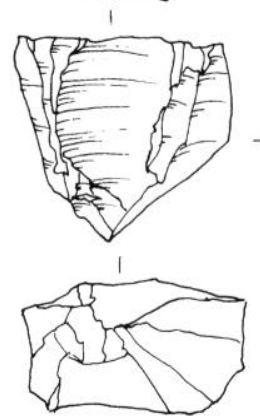

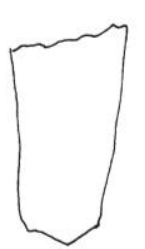
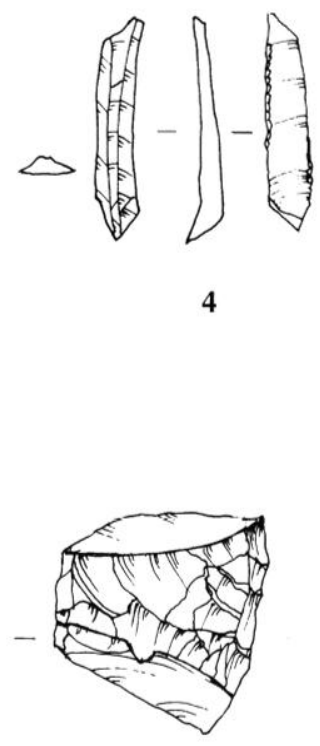

6

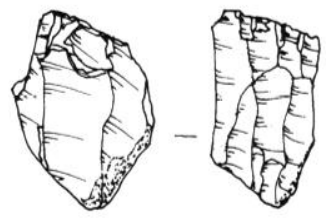

8
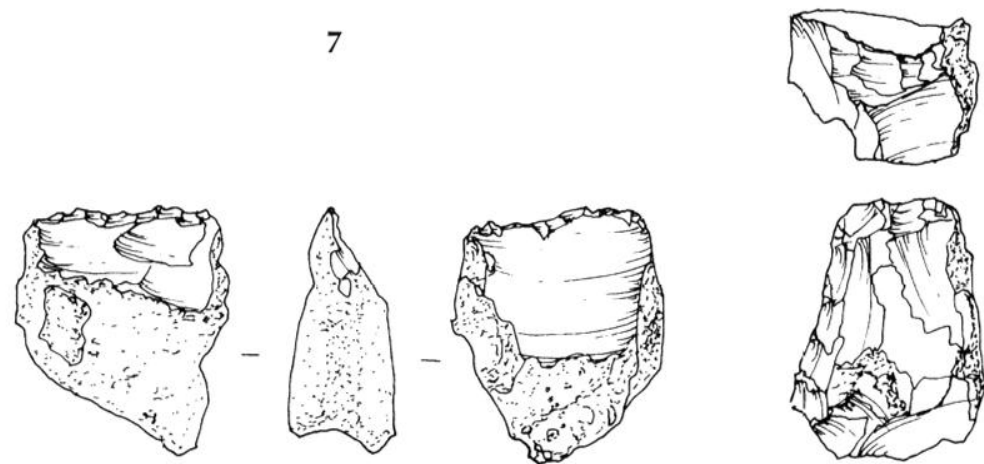

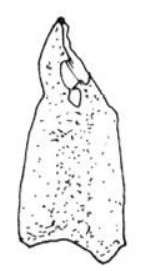

9

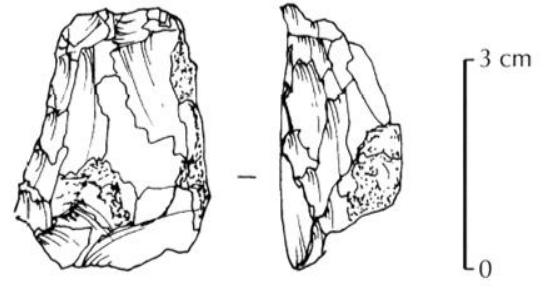

10

Fig. 52 - 1-10: Produits de débitage du locus 6 (dessin S. Saintot). 


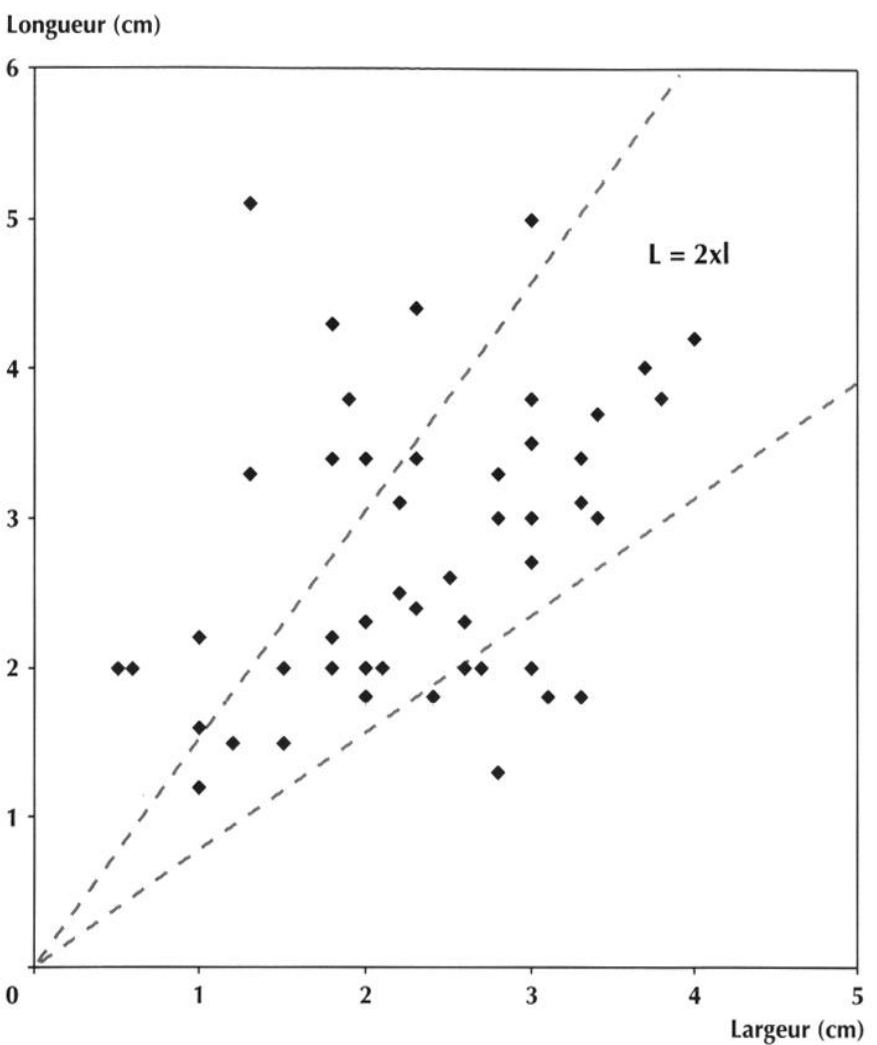

Fig. 53 - Indice d'allongement des produits lithiques de La Noue.

\section{Principales composantes de l'outillage lithique taillé}

Le tableau des décomptes total des outillages et du débitage (tabl. XXI) indique la prédominance des grattoirs ( $n=32$ ) sur un total de 63 outils, ce qui correspond, de même que pour la majorité des industries campaniformes, à plus de la moitié des produits aménagés à La Noue. Après les grattoirs, suivent les pièces esquillées $(n=9)$, les éclats retouchés $(\mathrm{n}=7)$ et les lamelles à retouches latérales $(n=4)$, les pièces encochées $(n=4)$, les pièces denticulées $(n=2)$ et les flèches $(n=2)$. Trois outils ne sont représentés que par un seul type chacun: un rctouchoir, un perçoir et un distal de lame de poignard. Parmi la totalité de l'outillage, seul un grattoir est brûlé.

\section{Les grattoirs}

Cinq principaux modèles ont été distingués dont:

- 17 grattoirs sur éclats corticaux ou non (fig. 47, $\mathrm{n}^{\circ} 10$; fig. $49, \mathrm{n}^{\text {os }} 1$ à 5 et 8 ; fig. $50, \mathrm{n}^{\text {os }} 1,3,5$ et 6 ; fig. $51, \mathrm{n}^{\text {os }} 4$ à 7 , 12 et 13$)$;

- 6 grattoirs unguiformes (fig. $49, n^{\circ} 6$; fig. $51, n^{\circ} 3$ et 8 à 11);

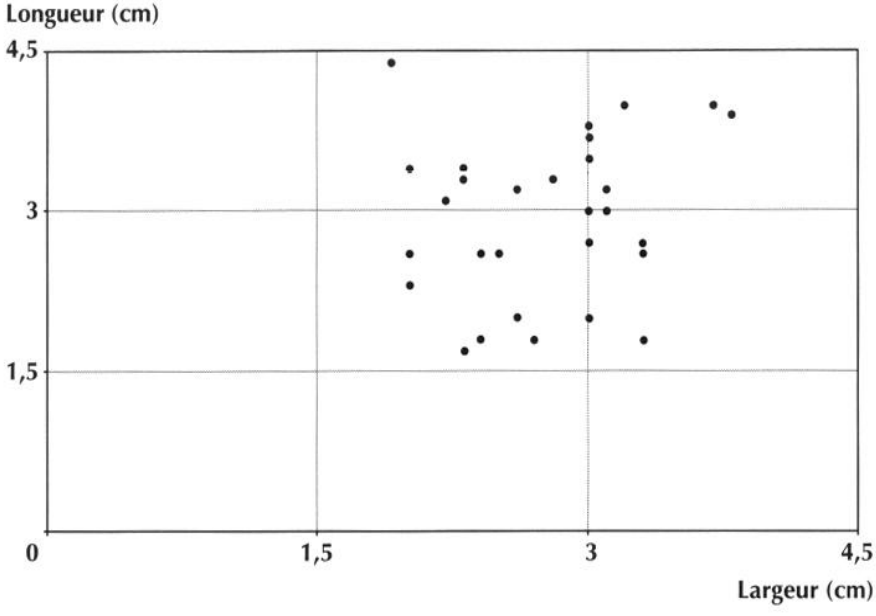

Fig. 54 - Indice d'allongement des grattoirs de La Noue.

- 5 grattoirs à front abrupt sur flancs de nucléus ou sur éclats épais (fig. $49, \mathrm{n}^{\text {os }} 7,9$ et 10 );

- 2 grattoirs doubles sur lames retouchées (fig. $47, \mathrm{n}^{\text {os }} 5$ et 9 ) ;

- 2 grattoirs sur supports esquillés (fig. $47, \mathrm{n}^{\circ} 8$; fig. 51, $\left.n^{\circ} 2\right)$.

L'importance des grattoirs sur éclats simples corticaux ou non, confirme une gestion opportuniste des produits de débitage pour le façonnage d'outil à partir d'un investissement technique moindre. À première vue, l'absence de standardisation systématique est de mise. L'indice d'allongement de ces produits montre toutefois une préférence pour des supports de petites dimensions, compris entre $1,5 \mathrm{~cm} \leq \mathrm{L} \leq 4,5 \mathrm{~cm}$ (fig. 54). Les modes de retouche employés pour le façonnage des fronts sont variés et se caractérisent par une retouche courte semi-abrupte, ou par une retouche écailleuse large abrupte.

\section{Les pièces esquillées}

Au nombre de 9 exemplaires, ces pièces se distinguent des autres outils par une grande variété de modèle. Les principaux éléments identifiés comptent:

- 5 éclats simples à esquillements bifaciaux alternes ou non (fig. 47, $\mathrm{n}^{\circ} 1$; fig. 48, n० 9; fig. 51, n०2);

- 2 éclats corticaux (fig. $52, n^{\text {os }} 5$ et 10 );

- 2 nucléus esquillés (fig. $48, \mathrm{n}^{\text {os }} 10$ et 11 ).

Cette variété d'outil, assez hétérogène se caractérise par une série d'esquillements alternes et opposés sur le produit de débitage le plus propice à l'emploi. Il s'agit d'outils $a$ posteriori, dont le support est modifié par esquillement après emploi et fonctionnement de celui-ci. L'intérêt réside dans 
l'utilisation du support qui devient outil: en coupant, en perçant, en sectionnant... (Sigaut, 1991). Une approche analytique réalisée sur cet outil revient à conclure à la polyvalence des pièces esquillées, réalisées sur le même site à partir de matériaux différents (Detrey, 2002, p. 190).

Ces outils sont majoritaires parmi les séries campaniformes d'Alle, Noir Bois et de Chenevez, Combe Villard; ceux-ci correspondent à $27,4 \%$ du total des artefacts manufacturés, alors qu'aux Florentins à Val-de-Reuil, aucun modèle de ce type n'a été recensé. Ces deux exemples non exhaustifs parmi l'ensemble des séries campaniformes étudiées, révèlent tout à fait la " part culturelle " concernant la présence ou l'absence des pièces esquillées, propre à chaque économie au sein même d'un site.

\section{Les éclats et les lames à retouches latérales}

Ces produits comptent 11 éléments à retouches latérales ou bilatérales, écailleuses courtes à scalariformes. L'emploi opportuniste de tout support apte à l'emploi semble prévaloir. Parmi les latéraux les plus caractéristiques, on compte:

- 3 lames courtes à retouches latérales continues ou irrégulières (fig. 46, $\mathrm{n}^{\text {os }} 1$ à 3 ) ;

- 7 éclats irréguliers à retouches latérales courtes (fig. 47 , $\mathrm{n}^{\circ} 6$; fig. $48, \mathrm{n}^{\circ} 3$ et 5 à 7 ; fig. $\left.51, \mathrm{n}^{\circ} 1\right)$;

- 1 lamelle à retouches bilatérales (fig. $52, \mathrm{n}^{\circ} 4$ )

- 1 éclat cortical de type grattoir-racloir (fig. 46, $\mathrm{n}^{\circ} 6$ ).

Comme déjà précisé auparavant, la volonté de produire des supports standardisés n'est pas de mise parmi cette série. Le $\mathrm{n}^{\circ} 6$ de la figure 46 , unique parmi la série de $\mathrm{La}$ Noue, comporte de larges retouches scalariformes semiabruptes sur la partie distale. Ce modèle n'est pas sans évoquer les exemplaires hybrides mis en évidence sur le site de Diguleville (Manche), tout à fait à l'ouest de la France (Letterlé, Verron, 1986).

\section{Les pièces encochées}

Deux modèles de ce type ont été identifiés (fig. $47, n^{\prime \prime} 7$; fig. $50, n^{\circ} 8$ ), ce qui reste anecdotique comparativement à l'ensemble des produits recensés.

\section{Les pièces denticulées}

Ces modèles comptent 2 exemplaires distincts et isolés typologiquement par rapport à la totalité de l'outillage (fig. $46, \mathrm{n}^{\circ} 9$; fig. $47, \mathrm{n}^{\circ} 6$ ). Les pièces encochées et denticulées, ubiquistes parmi les séries de la fin du Néolithique, ne constituent pas ici des marqueurs typologiques et culturels significatifs.

\section{Les flèches}

Deux modèles ont été définis comme tels, soit:

- une armature triangulaire perçante, peu investie techniquement, car elle est façonnée sur un proximal de lame courte à deux pans et elle est appointée par retouches écailleuses courtes et irrégulières, rasantes partielles sur le bord et sur le pan latéral droit du support (fig. $48, \mathrm{n}^{\circ} 1$ );

- une armature trapézoïdale tranchante, sur lamelle bitronquée à trois pans (fig. 50, $\mathrm{n}^{\circ} 4$ ).

Ces deux flèches, de facture peu soignée, ne sont probablement pas le fait de spécialistes. L'exemplaire triangulaire ne s'apparente pas aux modèles de flèches à base concave répandues en Europe centrale (Detrey, 2003, p. 200). D'un point de vue typologique, les deux armatures existent dans la région et au-delà, dès la fin du Néolithique moyen (NMB), (Bonnamour et al., 1990; Dufay-Galan, 1996) et perdurent au cours du Néolithique final, jusqu'au Campaniforme.

Les modèles losangiques pédonculés à ailerons droits ou récurrents font ici défaut, alors que la plupart des industries de référence comptent au minimum un ou deux exemplaires de ce type en fonction de l'importance des corpus étudiés (Saintot, 1999). Nous évoquerons plus loin les implications techniques et typologiques qui en découlent.

\section{Le retouchoir}

Un distal de lame épaisse comportant des esquillements et une plage émoussée a été identifié comme retouchoir ou briquet ce qui pourrait être confirmé ou infirmé à partir d'une analyse tracéologique.

\section{Le perçoir}

Un perçoir peu caractéristique typologiquement, car aménagé à partir d'un éclat appointé, est le seul produit de ce type reconnu parmi l'industrie (fig. $47, \mathrm{n}^{\circ} 1$ ).

\section{Le distal de lame de poignard}

Un distal de lame corticale dont l'extrémité est en forme d'ogive présente, sur le bord latéral droit, une retouche en écharpe par de fins enlèvements parallèles envahissants (fig. $48, n^{\circ} 2$ ). Bien que cette lame incomplète soit patinée, sa fabrication à partir d'un silex local du Crétacé est fort probable, et ceci, à partir de l'observation du cortex résiduel sur la face supérieure du support, tout à fait comparable à ceux qui affectent les autres silex corticaux recensés sur le site. Ce fragment pourrait appartenir à un poignard sur lame courte dont le cortex n'a pas été poli, à 
moins qu'il ne s'agisse d'une ébauche d'un modèle de ce type, fragmenté au cours du débitage. Les lames de poignard appartiennent généralement, en plus ou moins grande proportion, au panel des outillages campaniformes. Il s'agit en particulier de modèles losangiques en silex, importés des ateliers du Grand-Pressigny, ou, plus rarement, en matière première locale, qui sont dans de nombreux cas peu investis techniquement.

\section{L'outillage lithique non taillé}

Ce matériel a été traité séparément car il reste anecdotique $(n=5)$ parmi les artefacts lithiques recueillis sur le site (tabl. XXII). Signalons essentiellement, dans le locus 1, un lissoir sur galet (fig. 46, $\mathrm{n}^{\circ} 10$ ) et dans le locus 2, un polissoir à gorge concave peut-être employé pour le travail de l'os ou du bois (fig. 49, $\mathrm{n}^{\circ} 12$ ). Faiblement représentés, ces outils de façonnage ne sont toutefois pas significatifs d'un point de vue culturel, dans le cadre de notre étude. Ils confirment néanmoins la réalisation d'autres activités artisanales sur le site, parallèlement à celle de la taille du silex, comme le travail de l'os et du bois, souvent non conservés en milieu terrestre. On évoquera, par exemple, la présence d'une pointe en bois vraisemblablement utilisée comme arme de jet, retrouvée sur le site campaniforme de la rue RochetteChevrier, à Lyon $7^{\mathrm{e}}$ (Chastel et al., 2003), installé en contexte de plaine marécageuse. Cette pointe en bois durcie au feu comporte des traces de polissage résultant du façonnage sur polissoir à gorge...

\section{ANALYSE SPATIALE}

L'analyse spatiale a été réalisée à partir d'un choix de produits de débitage comprenant les nucléus, les lamelles, les éclats bruts et à partir d'une sélection d'outils significatifs, comme les grattoirs et les pièces esquillées.

La base de nos observations reposc sur les regroupcments de certains types d'outils, et sur le rapport proportionnel entre la quantité de matériel et les surfaces fouillées par locus (tabl. XXIII).

\section{Le locus 1}

La répartition des industries n'est pas particulièrement pertinente au sein de l'unité d'habitation car les silex sont distribués au sud, au centre et à l'est. De plus, le rapport entre le nombre de produits et la surface fouillée est de 0,2
Tabl. XXII - Répartition des pièces lithiques non taillées.

\begin{tabular}{|c|c|c|c|c|}
\hline Zone & UF & $\mathbf{N}^{\circ}$ & Détermination & Matière première \\
\hline 2 & 21 & $\mathbf{0}$ & mésial de galet & quartzite \\
\hline 2 & 13 & $\mathbf{0}$ & 1 galet thermofracté & quartzite \\
\hline 4 & 35 & $\mathbf{1 3 2}$ & 1 polissoir à gorge en molasse & molasse \\
\hline $\mathbf{1}$ & $\mathbf{2 2}$ & $\mathbf{8 4}$ & 1 lissoir s/galet de schiste ? & schiste \\
\hline $\mathbf{1}$ & $\mathbf{2 2}$ & $\mathbf{0}$ & 1 nodule de minerai & ? \\
\hline
\end{tabular}

Tabl. XXIII - Répartition de l'industrie lithique sur le site.

\begin{tabular}{|c|c|c|c|}
\hline & Industrie/Nombre & Surface $/ \mathrm{m}^{2}$ & Nombre $/ \mathrm{m}^{2}$ \\
\hline HS & 1 & 0 & 0 \\
\hline Zone 1 & 32 & 150 & 0,2 \\
\hline Zone 2 & 121 & 176 & 0,6 \\
\hline Zone 3 & 2 & 40 & 0,05 \\
\hline Zone 4 & 19 & 45 & 0,4 \\
\hline Zone 5 & 37 & 21 & 2 \\
\hline Zone 6 & 89 & 19 & 4,6 \\
\hline
\end{tabular}

silex par mètre carré. Trois supports laminaires débités à la percussion indirecte, sans remontage possible, sont regroupés en UF 19 (fig. $46, \mathrm{n}^{\text {os }} 1$ et 2 et fig. $47, \mathrm{n}^{\circ} 5$ ). Il s'agit là probablement d'une suite de produits sortis d'un même bloc par le même tailleur. Sur la totalité des silex recensés, au nombre de 32 individus, aucun raccord n'a pu être réalisé. Parmi les outils faiblement représentés $(n=7)$, 2 grattoirs sont regroupés au centre de la maisonnée, à la périphérie d'une concentration de céramique.

\section{Le locus 2}

Ce locus a livré 121 produits lithiques taillés, dont une vingtaine de produits retouchés et aménagés. Une vingtaine de silex au total sont brûlés. Le rapport entre le nombre de produits et la surface fouillée est de 0,6 silex par mètre carré. Parmi les produits de débitage, 4 nucléus globuleux (UF 16 et UF 27) sur 8 produits de ce type recensés, sont regroupés au centre de l'aire principale de concentration de mobilier. Un nucléus à lamelle, préalablement chauffé, provient de l'UF 24, mais il s'agit-là du seul nucléus dont la texture est modifiée par la chauffe. L'unique percuteur sur galet de chaille provient d'ailleurs de ce locus riche en nucléus et en produits de débitage. Dans l'UF 13, une production d'éclats débités à la percussion directe sur enclume a été décrite à partir de trois éclats corticaux en « tranche d'orange ". Douze grattoirs subdivisés en deux groupes typomorphologiques, regroupés suivant trois aires de 
concentration de mobiliers, s'inscrivent probablement dans plusieurs espaces d'activités spécialisées (fig. $49, \mathrm{n}^{\text {os }} 1$ à 10 ). Enfin, deux outils dont une armature de flèche triangulaire et une ébauche de lame de poignard à retouches subparallèles ont été recueillis dans la même unité de fait (UF 13) (fig. $48, n^{\text {os }} 1$ et 2$)$. Malgré le nombre d'esquilles $(n=16)$, d'éclats $(n=56)$ et de pièces techniques $(n=10)$ relativement élevé, aucun remontage n'a pu être réalisé, ce qui induirait une sélection de certains produits utilisés à l'extérieur des unités d'habitation ou peut-être même un nettoyage systématique des espaces domestiques.

\section{Le locus 4}

Le rapport entre le nombre de produits et la surface fouillée est de 0,4 silex par mètre carré. De même que le locus 1, le locus 4 n'a pas livré de nombreux produits manufacturés $(n=19)$; toutefois 3 grattoirs sur éclat proviennent de ce même secteur, laissant supposer une activité spécialisée à cet emplacement (fig. $50, \mathrm{n}^{\mathrm{os}} 3,5$ et 6 ). Un mésial de lame courte bitronquée, peut-être employé comme armature tranchante, constitue avec l'exemplaire du locus 2 (fig. 48, $\mathrm{n}^{\circ} 1$ ) le second élément pouvant être utilisé comme armature de flèche. Aucun parallèle technique ou typologique ne peut néanmoins être réalisé entre ces deux exemplaires, d'autant qu'il n'est pas certain qu'il s'agit d'armes de jet!

\section{Le locus 5}

Ce locus compte 4 fosses, dans lesquelles 37 silex ont été recueillis, les outils étant minoritaires dans ce contexte $(n=7)$. Le rapport entre le nombre de produits et la surface fouillée est de 2 silex par mètre carré. Les éléments taillés exhumés de ces fosses, dont 9 silex sont brûlés, correspondent vraisemblablement à du matériel détritique; néanmoins, nombre d'entre eux présentent les mêmes traits typo-techniques. C'est en particulier dans l'UF 12 que les principaux outils ont été recueillis, ceux-ci ayant tous la particularité d'être esquillés sur la face inférieure (fig. 46, $\mathrm{n}^{\text {os }} 3,6$ et 9 ; fig. $47, \mathrm{n}^{\text {os }} 2,8$ et 9 ; fig. $50, \mathrm{n}^{\text {os }} 1$ et 2 ). Ce trait technique montre que ces produits sont issus du même bloc, peut-être débités sur enclume au percuteur dur, et produits par la même main. À noter la présence d'un éclat de chaille, situé non loin du locus 2 où un percuteur employé dans un matériau identique a été recueilli.

\section{Le locus 6}

Il s'agit de l'aire la plus excentrée des six locus mis au jour lors de la fouille. Le rapport entre le nombre de produits et la surface fouillée est de 4,6 silex par mètre carré. Une aire de concentration de matériel regroupant 89 produits au total, compte 11 grattoirs et 4 pièces esquillées (fig. 51 et 52). Parmi ces silex, 7 produits de débitage sont brûlés. Si l'on raisonne par unité d'habitation, des associations morphologiques apparaissent avec le groupe des grattoirs unguiformes $(n=5)$, et avec celui des grattoirs à front large sur éclats courts $(n=5)$. Ces appariements sous-tendent des activités spécialisées au sein des cellules d'habitation. Notons dans ce cas le complément d'information qu'apporterait une analyse tracéologique sur ces outils, sachant qu'à Noir Bois, cette approche a permis de conclure au travail des peaux pour bon nombre des grattoirs étudiés (Astruc, 1997). Trois nucléus présentent également les mêmes stigmates techniques révélant des similitudes de facture. Parallèlement à ceci, une petite série de lamelles tirées à la pression s'inscrit dans une chaîne opératoire standardisée. L'ensemble de ces paramètres techniques comparables confirme une production spécialisée dans le locus 6 , fait qui ne se retrouve pas ailleurs aussi distinctement.

Du sud au nord, la répartition du matériel lithique n'est pas aléatoire puisque plusieurs assemblages de produits de débitage et d'outils ont été mis en évidence, et ceci particulièrement dans les locus 2,5 et 6 , induisant une production intentionnelle vouée à une même activité.

Les regroupements de certains assemblages de matériel recouvrent plusieurs paramètres, d'ordre technique et typologique, qui caractérisent l'identité même du groupe campaniforme de La Noue. Certains produits, typologiquement identiques et de facture semblable, présentant des « airs de famille " similaires, sont le fait des mêmes artisans tailleurs, dont il est difficile d'évaluer le degré de spécialisation puisque la plupart des chaînes opératoires sont incomplètes. Si tout le monde ne taille pas sur le site, un ou deux artisans peuvent produire un débitage plus ou moins élaboré au sein de chacune des maisonnées. De plus, l'association de grattoirs de même facture, en particulier dans les locus 2 et 6 , induit une production intentionnelle d'outils spécifiques, vouée à une même activité. Ainsi, la présence d'aires spécialisées peut être évoquée à La Noue.

S. S. 


\section{BILAN SUR L'ORGANISATION DU MOBILIER SUR LE SITE}

Les études menées sur le mobilier du site de La Noue conduisent à préciser plusieurs données relatives au fonctionnement du site.

En ce qui concerne la contemporanéité des unités d'habitation, les liaisons effectuées entre tessons (collages et regroupements) ont montré que les locus 1, 2 et 4 devaient, au moins dans une des phases de leur occupation, avoir fonctionné en synchronie. L'étude des matières premières des vases démontre la même synchronie pour les locus 1,2 et 6 , du fait de la proximité des composantes de plusieurs vases de ces trois locus. Dans l'industrie lithique, comme dans la série céramique, rien ne permet de différencier plusieurs ensembles chronologiques. Il n'y a donc pas de gros décalages chronologiques entre les unités d'habitation révélées par la fouille, mais il est impossible de proposer une chronologie plus fine.

En revanche, plusieurs faits mettent en lumière des différences d'ordre fonctionnel entre les locus. La concentration d'outils et leur association plaident en faveur d'activités spécialisées au sein des locus 2 et 6 . La répartition des assemblages céramiques montre, quant à elle, que les locus 2 et 5 sont davantage voués à des fonctions de stockage, alors que dans les locus 1 et 6 la céramique de présentation et de préparation de l'alimentation domine. C'est également des locus 2 et 5 que proviennent les rares restes fauniques.

Enfin, les éléments céramiques exogènes ou d'inspiration extra-régionale sont concentrés dans les locus 2 et 6 , ceux-ci se distinguant déjà par une industrie lithique vouée à des activités particulières. Peut-on y voir un lien?

F. C., S. S. et L. S.

\section{LE SITE DE LA NOUE DANS LE CONTEXTE RÉGIONAL ET EXTRA-RÉGIONAL}

\section{LE CAMPANIFORME \\ EN BOURGOGNE ORIENTALE ${ }^{9}$}

\section{HISTORIQUE DES RECHERCHES}

L'historique de la recherche sur le Chalcolithique en Bourgogne commence dans le milieu du XIX ${ }^{e}$ siècle avec les

9. Nous avons exclu l'ouest de la région dans cet inventaire car il participe d'une autre unité culturelle et ce, tout au long du Néolithique. premières fouilles intensives pratiquées sur les grands sites de hauteur. Ces fouilles sont souvent menées par des ouvriers travaillant pour des propriétaires qui souhaitaient former des collections, et non pratiquer une archéologie basée sur la recherche et la compréhension des vestiges. C'est dans ce contexte que la collection Loydreau a été constituée lors des premières fouilles du site du Camp à Chassey en 1870. Les 2 tessons campaniformes contenus dans cette collection apportent cependant l'un des tout premiers témoignages sur la période en Bourgogne. Ils n'ont pas été reconnus comme tels à une époque où la chronologie de la Préhistoire n'en était qu'à ses premiers pas. Ensuite, la recherche archéologique évolua et les premières fouilles documentées sont dues aux sociétés archéologiques et aux archéologues amateurs de la première moitié du $\mathrm{xx}^{\mathrm{e}}$ siècle. Les fouilles de la nécropole mégalithique de Fleurey-sur-Ouche par Socley en 1910 et de la grotte de Rochechèvre à Ternant par E. Guyot et la brigade archéologique de Bourgogne en 1939 en sont l'illustration. Au gré de ces fouilles, qui n'intéressaient en général que les sépultures mégalithiques, quelques grottes et sites de hauteur des plateaux bourguignons, la documentation s'accumula sur le Chalcolithique qui n'en demeura pas moins une période chronologique méconnue. Il fallut attendre les années 1950 avec le démarrage des fouilles d'Ouroux-sur-Saône et la reprise des fouilles sur les sépultures mégalithiques au début des années 1960 par l'abbé Joly pour que la recherche sur le Chalcolithique et le Campaniforme puisse connaître un réel essor. Cette époque coïncide avec la publication des premières synthèses à l'échelon national (Riquet et al., 1963; Treinen, 1970). Les travaux engagés par J.-P. Thévenot sur le site du Camp à Chassey apporteront la seule stratigraphie contenant un niveau campaniforme connue en Bourgogne. Les années 1980 voient l'intensification des opérations d'archéologie préventive qui offrent un complément indispensable à la compréhension du Chalcolithique en Bourgogne oricntalc avec la découverte de sites de plaine, souvent situés dans des secteurs peu favorables à la prospection. C'est dans ce contexte qu'est découvert le site de La Noue en 1996, mais aussi les sites de Corvée-Rateau à Beaune, des Allées Cavalières à Quétigny, de Larrivoux à Pluvet et des Quétignères à Longvic, dont il sera question dans le paragraphe qui suit ${ }^{10}$. Nous reviendrons également sur les

10. Nous tenons à remercier les différents responsables d'opération qui nous ont communiqué ces informations inédites. 


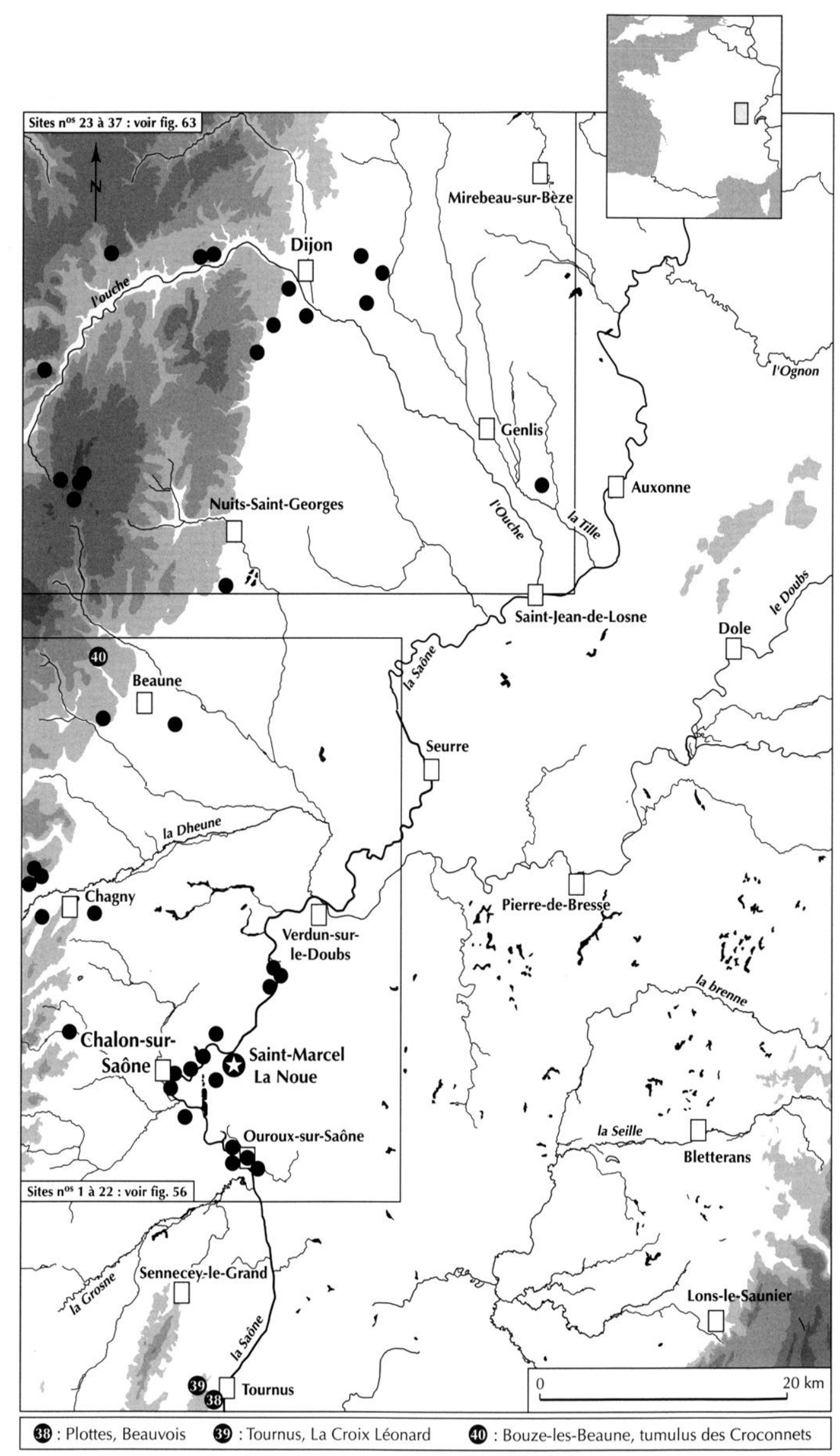

Fig. 55 - Carte du Campaniforme en Bourgogne orientale (carte F. Ducreux). Voir aussi les figures 56 et 63. 
découvertes anciennes, nécessaires à une compréhension globale de l'occupation. L'inventaire s'articule entre les deux régions principales que sont le Dijonnais, avec le débouché de la vallée de l'Ouche et la vallée des Tilles, e.t le. Chalonnais avec la confluence de la Dheune et de la Saône (fig. 55).

F. D.

\section{Le Campaniforme en SAÔNE-ET-Loire et dans le Chalonnais}

Dans cet inventaire, seuls sont pris en compte les sites ayant fourni du mobilier attribuable au Campaniforme (fig. 56). Les sites attribués précisément au Bronze ancien, comme la nécropole de la Bergère à Verzé, n'y figureront donc pas ${ }^{11}$.

\section{Les découvertes récentes}

Beaune, Corvée Rateau. La découverte du site de Corvée Rateau à Beaune est due aux travaux réalisés en 1998 sur le tracé de la future rocade est de l'agglomération. Plusieurs phases d'occupation, s'étendant sur environ $30000 \mathrm{~m}^{2}$, sont datées du Chalcolithique au Bronze final IIIb. L'occupation chalcolithique est matérialisée par une fosse située près d'un petit bâtiment sur poteaux. Deux tessons décorés et un bord de récipient à cordon lisse y ont été découverts (fig. 57 , $\mathrm{n}^{\text {os }} 7$ à 9). Les structures et le bâtiment sur poteaux situés près de la fosse ne peuvent pas être datés avec précision puisqu'ils n'ont pas livré de mobilier.

F. D.

\section{Les découvertes anciennes}

\section{L'habitat}

Chassey, Le Camp. Parmi l'abondant matériel que cet habitat a livré depuis le $\mathrm{XIX}^{\mathrm{e}}$ siècle figurent des tessons campaniformes du standard, ainsi qu'un brassard d'archer à deux perforations et un doigtier en os (fig. $58, n^{\text {os }} 1$ à 8 ). Cet ensemble provient des fouilles anciennes du docteur Loydreau (Combier, 1962).

Crissey, Le Mont. Comme toujours en Val de Saône, cet habitat est situé sur un tertre sablonneux, en limite de la

11. Nous remercions vivement les collègues qui nous ont aidé dans cette étude: L. Bonnamour, J.-P. Thévenot et J. Duriaud pour avoir fourni d'utiles renseignements sur leurs travaux; $C$. Michel qui a réalisé une partie des illustrations; C. Bontemps qui nous a laissé toute liberté de faire état du matériel inédit de ses fouilles à Ouroux, Le Petit Bois. plaine alluviale et de la terrasse de $15 \mathrm{~m}$, à une altitude de $176 \mathrm{~m}$ qui le mettait à l'abri des crues. Un espace restreint a livré 16 fragments de vases campaniformes, une écuelle carénée à deux mamelons perforés longitudinalement, une hachette polie et plusieurs armatures (fig. 59a). Douze de ces fragments, qui appartiennent vraisemblablement à un même gobelet, portent des lignes parallèles d'impressions au peigne. Les autres tessons, également imprimés au peigne, sont ornés d'une ligne de chevrons, placée entre deux groupes de lignes horizontales, et un décor en échelle (Gros, 1966).

Dracy-le-Fort, Les Varennes. Ce site de surface, en bordure immédiate de l'Orbize, affluent de la Saône, a été occupé depuis le Paléolithique moyen. Il fait partie de ces nombreuses stations préhistoriques qui jalonnent le bord des rivières jusqu'à la Loire. Dans un terrain sablonneux, maintes fois remanié par les labours, l'exploitant a ramassé une industrie néo-chalcolithique, dont des pointes bifaciales et un poignard en métal cuivreux qui n'a pas fait l'objet d'une analyse (fig. $60, \mathrm{n}^{\circ} 4$ ). Cette lame losangique martelée est caractéristique des poignards campaniformes (Armand-Calliat, 1962; Bonnamour, 1969). La céramique n'a malheureusement pas été conservée par l'inventeur du site.

Épervans, Les Lacs. Au pied de la terrasse de $15 \mathrm{~m}$, dans un site occupé du Néolithique à la période gallo-romaine, une pointe, probablement en cuivre, a été découverte lors de prospections (Bonnamour, 1975). Ovale, à soie longue de section irrégulière, entièrement martelée, elle est à rapprocher des pointes de Palmela (fig. $60, \mathrm{n}^{\circ} 1$ ). Elle a été trouvée à $500 \mathrm{~m}$ du cours de la Saône, marquant ainsi la première découverte de ce type d'objet hors des zones littorales dans le couloir Rhône-Saône.

Gergy, La Maladière. Sur la première terrasse de la Saône, à $183 \mathrm{~m}$ d'altitude, L. Bonnamour a ramassé, dans un espace restreint, trois fragments d'un ou de plusieurs vases décorés de lignes horizontales imprimées au peigne et deux armatures, l'une à pédoncule et ailerons et l'autre losangique (fig. 61a). Les conditions de découverte permettent d'envisager en ce lieu la présence d'une sépulture qui aurait été détruite par les travaux agricoles (comm. pers. L. Bonnamour).

Gergy-Bougerot, La Prime. Dans la même situation géologique et topographique que La Maladière, dans un secteur occupé du Néolithique au Moyen Âge, il a été trouvé un tesson décoré d'une ligne d'impressions surmontée de traits verticaux (fig. $61 \mathrm{a}, \mathrm{n}^{\circ} 4$; comm. pers. L. Bonnamour). 


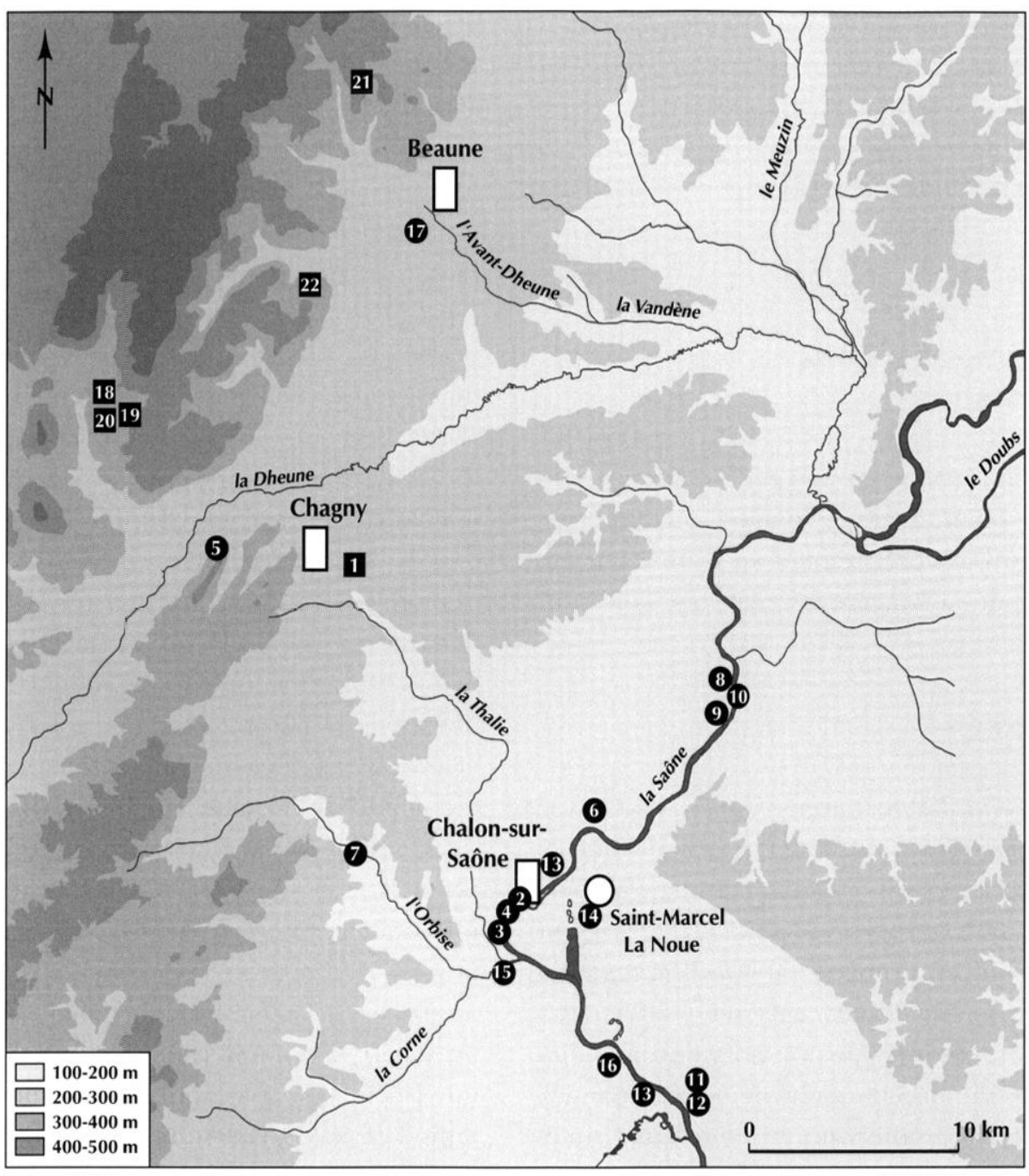

\begin{tabular}{|cl|}
\hline 1 Chagny, Vertempierre & 13 Ouroux-sur-Saône, Le Petit Bois \\
2, 3, 4 Trouvailles sans contexte dans la Saône : & 14 Saint-Marcel, Le Breuil \\
un poignard type Soyons, une épée et une hache plate & 15 Saint-Rémy, Moulin de Droux \\
5 Chassey, Le Camp & 16 Varennes-le-Grand, La Maison Blanche \\
6 Crissey, station du Mont & 17 Beaune, Corvée Rateau \\
7 Dracy-le-Fort & 18 La Rochepot, Les Épenottes \\
8 Gergy, La Maladière & 19 La Rochepot, La Chaume \\
9 Gergy-Bougerot, La Prime & 20 La Rochepot, La Garrenne \\
10 Gergy, un poignard & 21 Bouze-les-Beaune, Les Croconnets \\
11 Ouroux-sur-Saône, Carrière des Boulets & 22 Volnay, La Pierre Brûlée \\
12 Ouroux-sur-Saône, Grand Bois et Taillis & $\mathbf{6}$ habitat 20 sépulture \\
\hline
\end{tabular}

Fig. 56 - Carte du Campaniforme dans le Chalonnais et la vallée de la Dheune (carte F. Durreux).

Igé, berge du Talenchant. Dans la coupe de la berge du ruisseau de Talenchant, il a été trouvé deux armatures, l'une losangique et l'autre à base droite, et un fragment de gobelet décoré en arête de poisson (Combier, 1959; fig. 58, $\mathrm{n}^{\text {os }} 19$ à 21).

Ouroux-sur-Saône, carrière des Boulets. Des extractions de sable dans la plaine alluviale, au bas de la terrasse de $15 \mathrm{~m}$, ont détruit un monticule dans lequel se trouvait un habitat voisin, bien que distinct, de ceux du Grand Bois et du Petit Bois. De nos jours, ce site n'existe plus, mais, avant sa disparition, les prospections de A.-C. Gros et celles de L. Bonnamour ont permis de récolter de nombreux vestiges, en particulier une dizaine de fragments de vases campaniformes décorés au peigne et un seul à la cordelette 


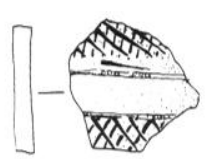

1

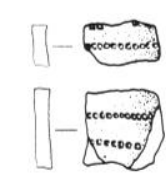

2

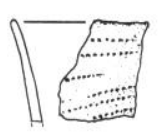

3

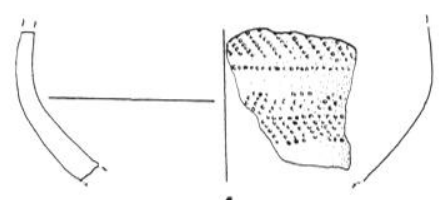

4

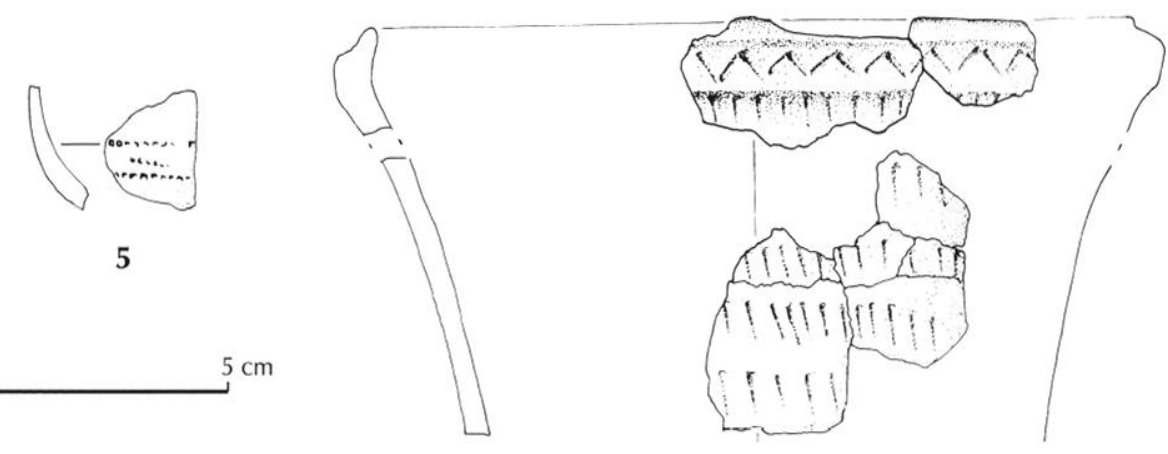

6

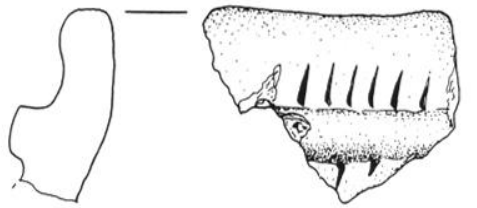

7

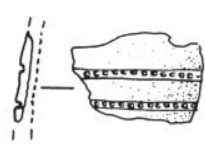

8

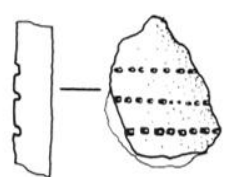

9

Fig. 57 - Mobilier campaniforme: 1-6, site de Quétigny, Les Allées Cavalières (dessin F. Ducreux et F. Gaucher); 7-9, site de Beaune, Corvée Rateau (dessin F. Ducreux).

(fig. 61b). Le mobilier lithique comprend des lames, des grattoirs, des microlithes, des éclats retouchés, une scie caractéristique à trois encoches et des armatures à base arrondie ou à pédoncule, le tout constituant le fonds commun des habitats des berges de la Saône chalonnaise qui ont été occupées sans interruption depuis le Néolithique jusqu'aux périodes historiques (Gros, Gros, 1968).

Ouroux-sur-Saône, habitat de La Berge, lieu-dit Le Grand Bois et Le Taillis. Cet important site, révélé par des prospections en 1952, est établi sur la rive gauche de la Saône, à environ $1 \mathrm{~m}$ de profondeur. Plusieurs fouilles et sondages ont précisé qu'il s'agissait des vestiges d'un village chalcolithique qui s'étendait sur l'espace compris entre la rivière et la basse terrasse, dans le lit majeur de la rivière, à 174 et 175 m d'altitude (Gros, Thévenot, 1957). Les recherches de surface ont permis la découverte de plusieurs centaines d'armatures de flèches perçantes, dont certaines ogivales courtes et à ailerons équarris, de grands poignards de type pressigniens, des scies à encoches, des microlithes de formes géométriques, etc. La céramique domestique comprend des grands récipients à fond arrondi et des bols non décorés, munis seulement d'éléments de préhension (Thévenot, 1973). Il a également été trouvé quelques tessons de gobelets campaniformes ornés d'échelles incisées ou décorés au peigne (fig. 61c). Signalons que deux tessons campaniformes, un racloir, l'extrémité d'un poignard et une pointe de flèche à pédoncule et ailerons équarris ont été trouvés groupés dans le même niveau (Combier, 1959). Les tessons sont généralement épais, de fabrication peu soignée, à l'exception de certains fragments rouges ou orangés décorés au peigne dans le style international, et dont la pâte est fine.

Ouroux-sur-Saône, Le Petit Bois. L'ouverture d'une gravière dans le lit majeur de la Saône (174 m d'altitude) a mis au jour une couche néo-chalcolithique, à $400 \mathrm{~m}$ environ du site du Grand Bois, dont il est séparé par une levée récente de terre, établie pour le passage d'une route départementale. La situation topographique et géologique des deux sites étant exactement similaire, il est permis de penser qu'il s'agit d'un seul et même habitat. Les travaux de C. Bontemps font état de foyers, d'aires de débitage et d'un trou de poteau (Bontemps, 1997). Le matériel recueilli est 


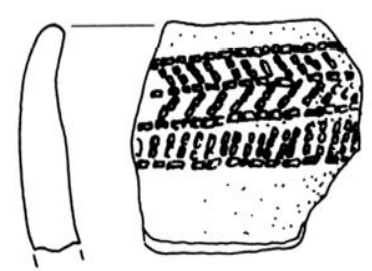

1

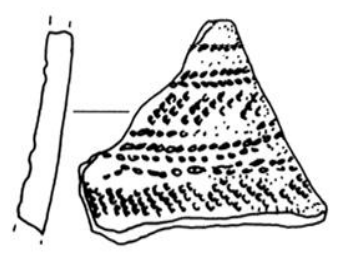

2

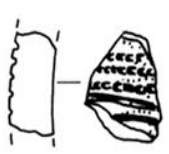

3

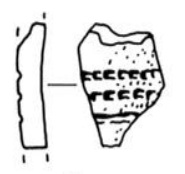

4

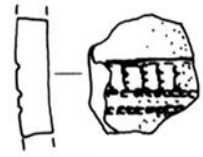

5

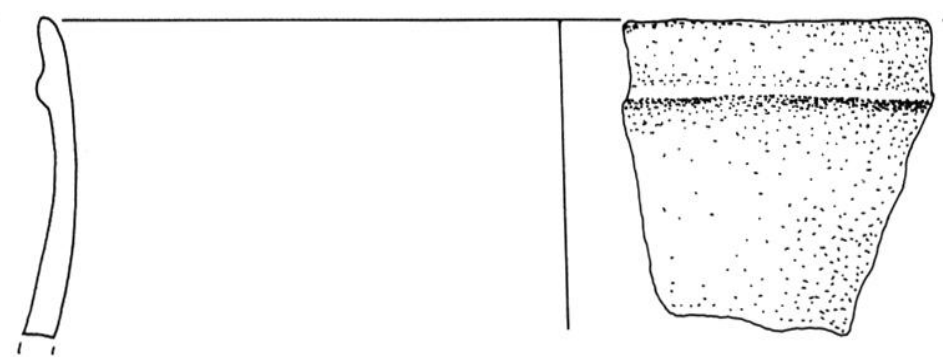

6
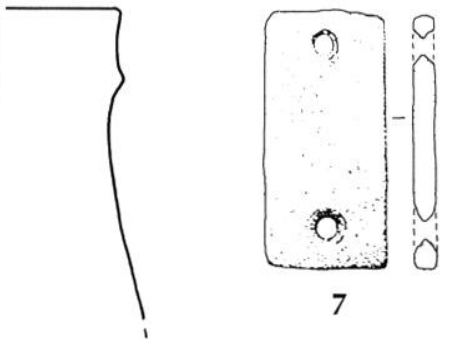

0 $5 \mathrm{~cm}$
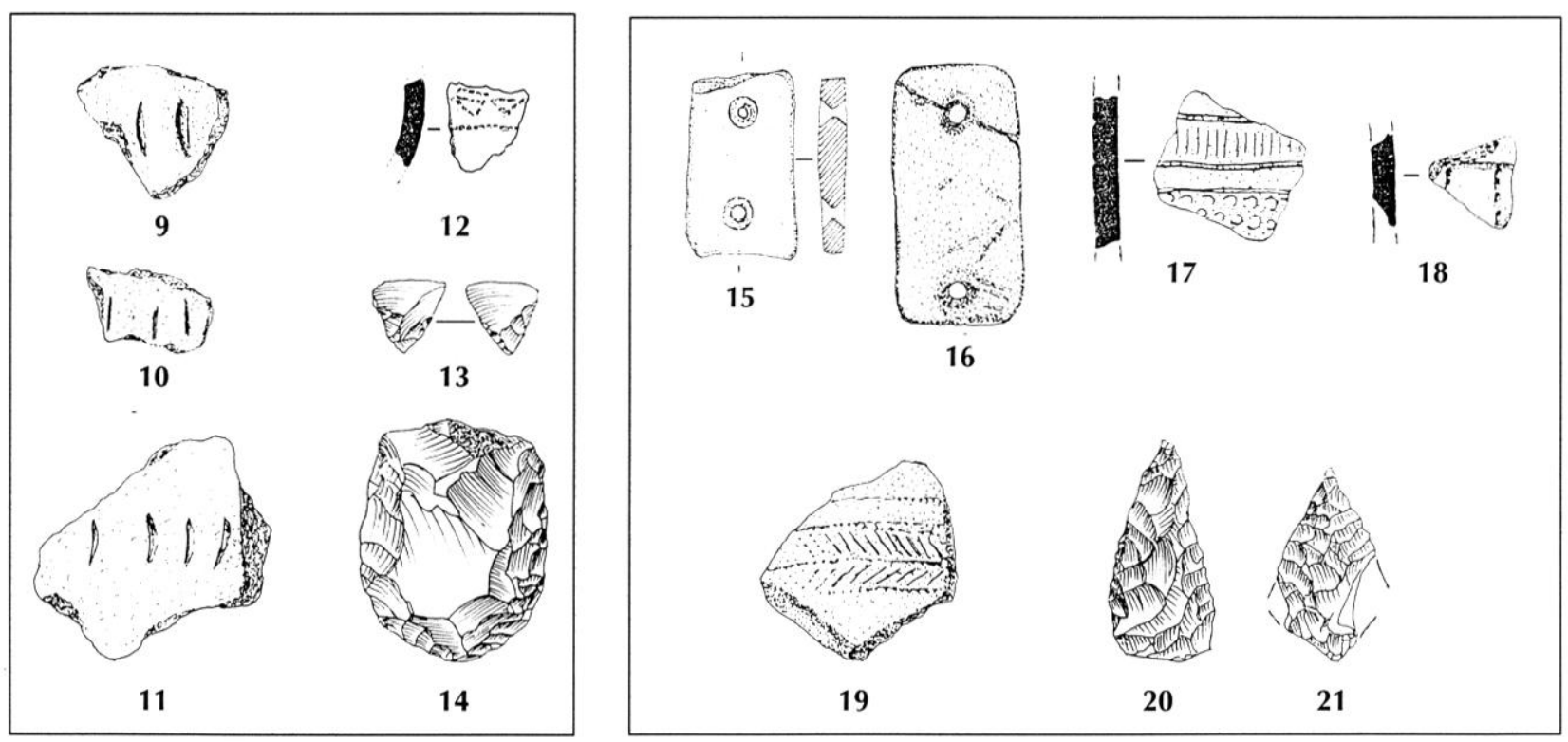

Fig. 58 - Mobilier campaniforme provenant de Saône-et-Loire et du Rhône: 1-8, site de Chassey, Le Camp (1, 2, 6-8, collection Loydreau; 3-5, fouilles J.-P. Thévenot de La Redoute, niveau 5; dessin F. Ducreux, O. Gaiffe, A.-C. Gros et J.-P. Thévenot); 9-14, Varennes-le-Grand, La Maison Blanche (dessin A.-C. Gros); 15, Saint-Romain, Le Verger (dessin A.-C. Gros, d'après S. Grappin); 16, grotte d'Azé (dessin A.-C. Gros, d'après photo de Dravet); 17, Saint-Georges-de-Reneins, Boitrait (dessin A.-C. Gros, d'après D. Philibert); 18, Saint-Rémy, Le Moulin de Droux (dessin A.-C. Gros); 19-21, Igé, La Berge du Talenchant (dessin A.-C. Gros, d'après A. Barthélemy). 


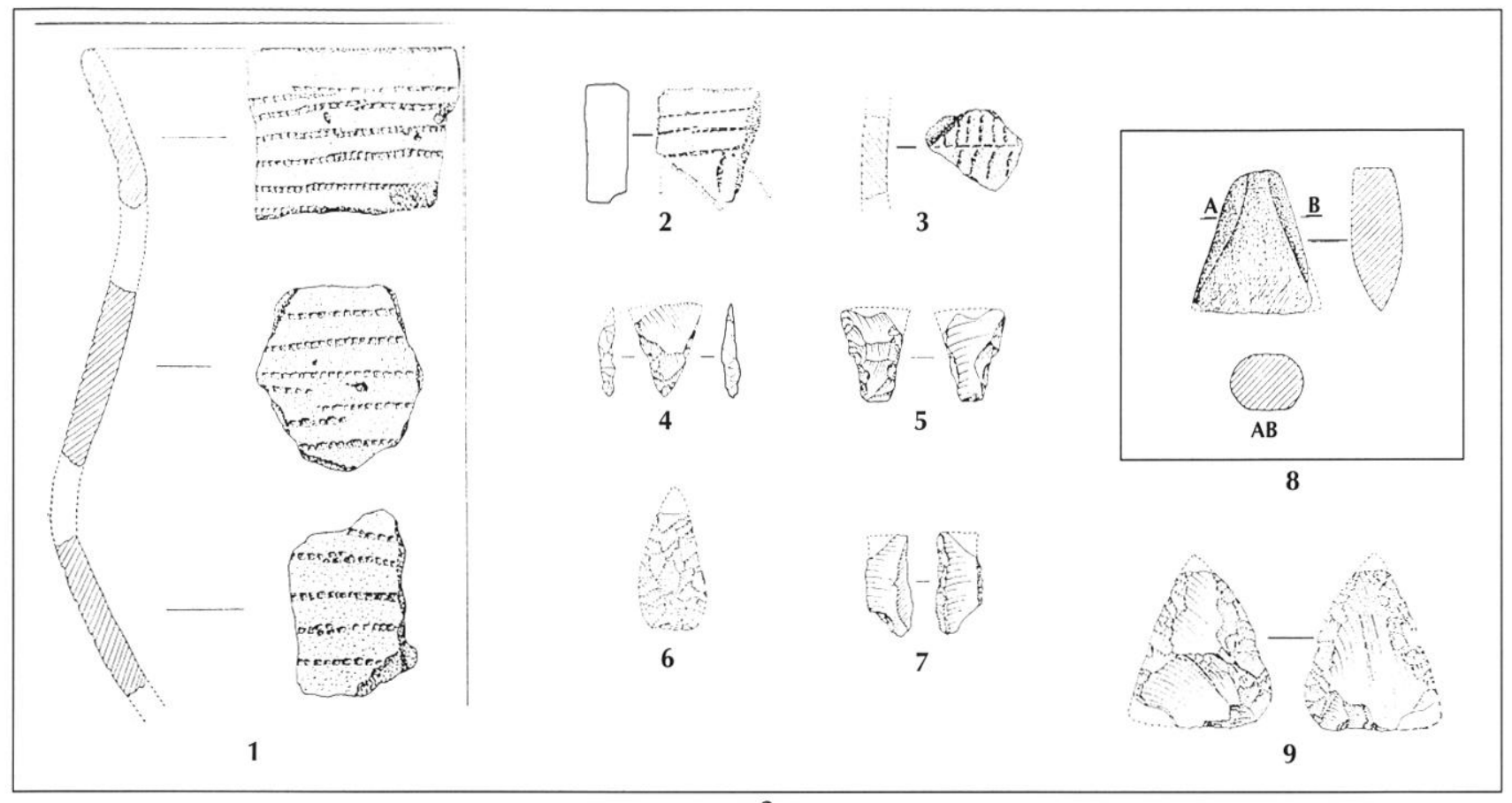

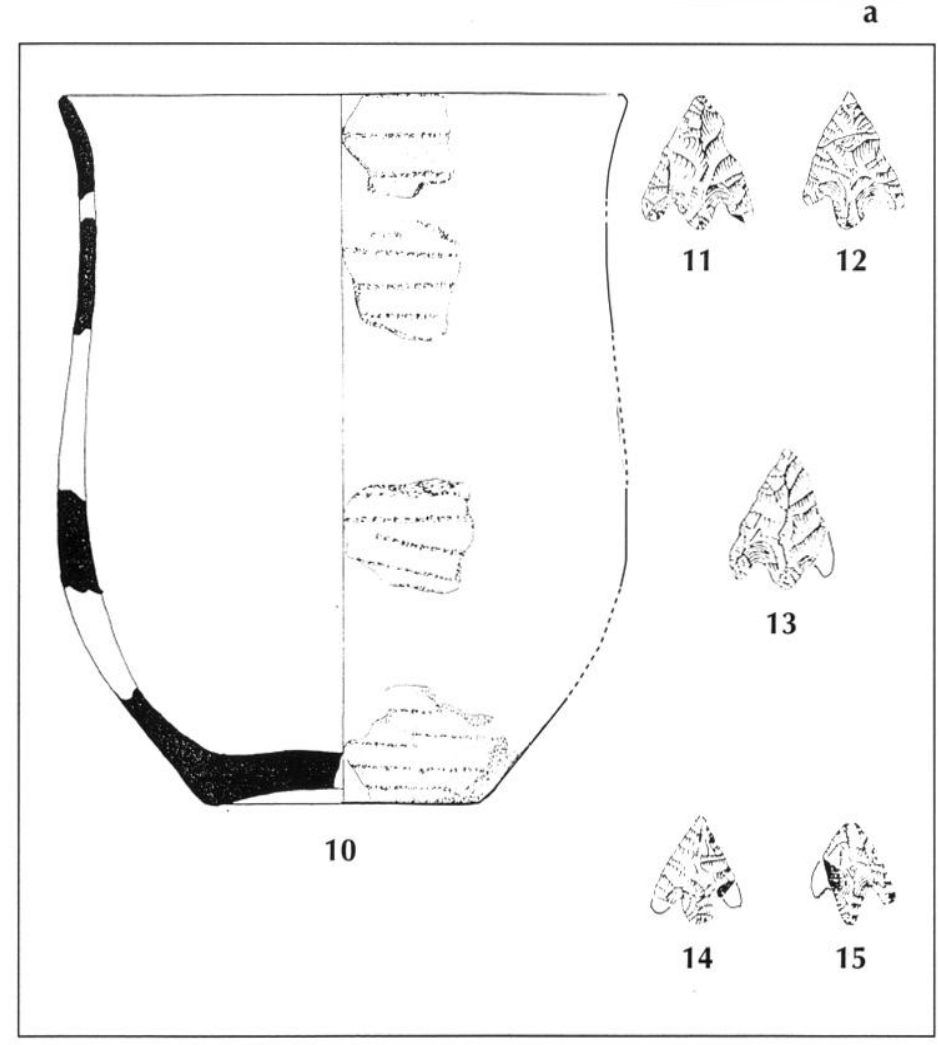

b
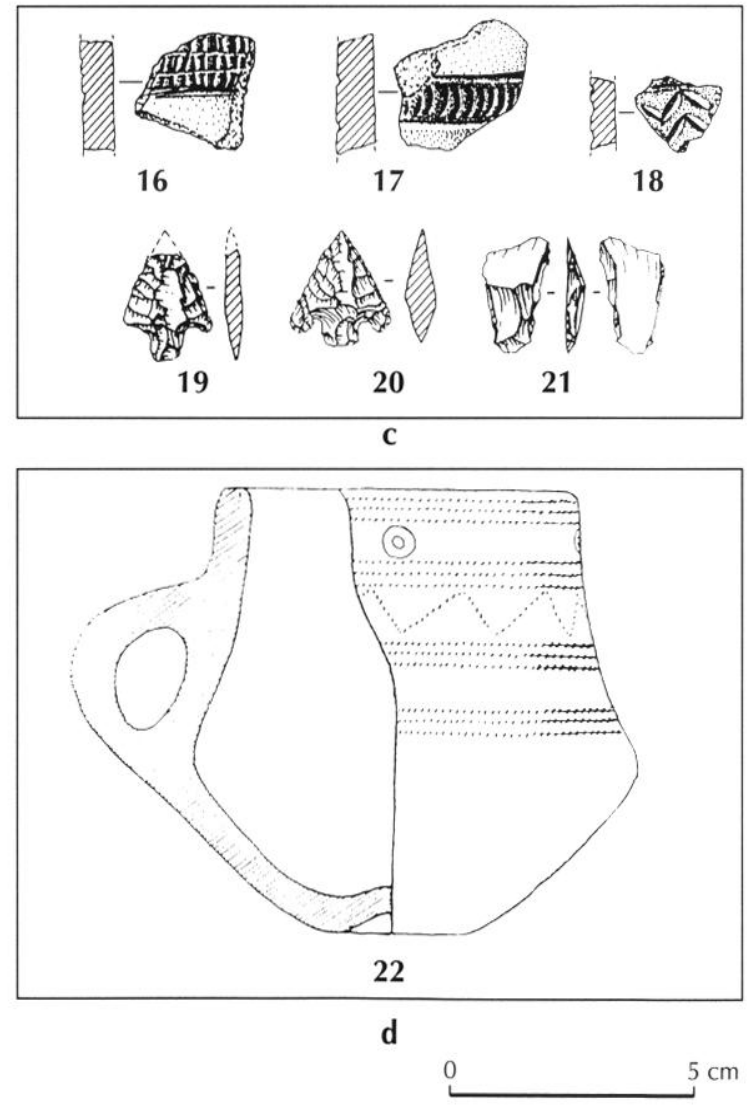

Fig. 59 - Mobilier campaniforme provenant de Saône-et-Loire: a, 1-9, Crissey, Le Mont (dessin A.-C. Gros); b, 10-15, Saint-Marcel, Le Breuil (dessin L. Bonnamour); c, 16-21, Tournus, La Croix Léonard (dessin J. Duriaud); d, 22, La Truchère (dessin G. Gaudron). 

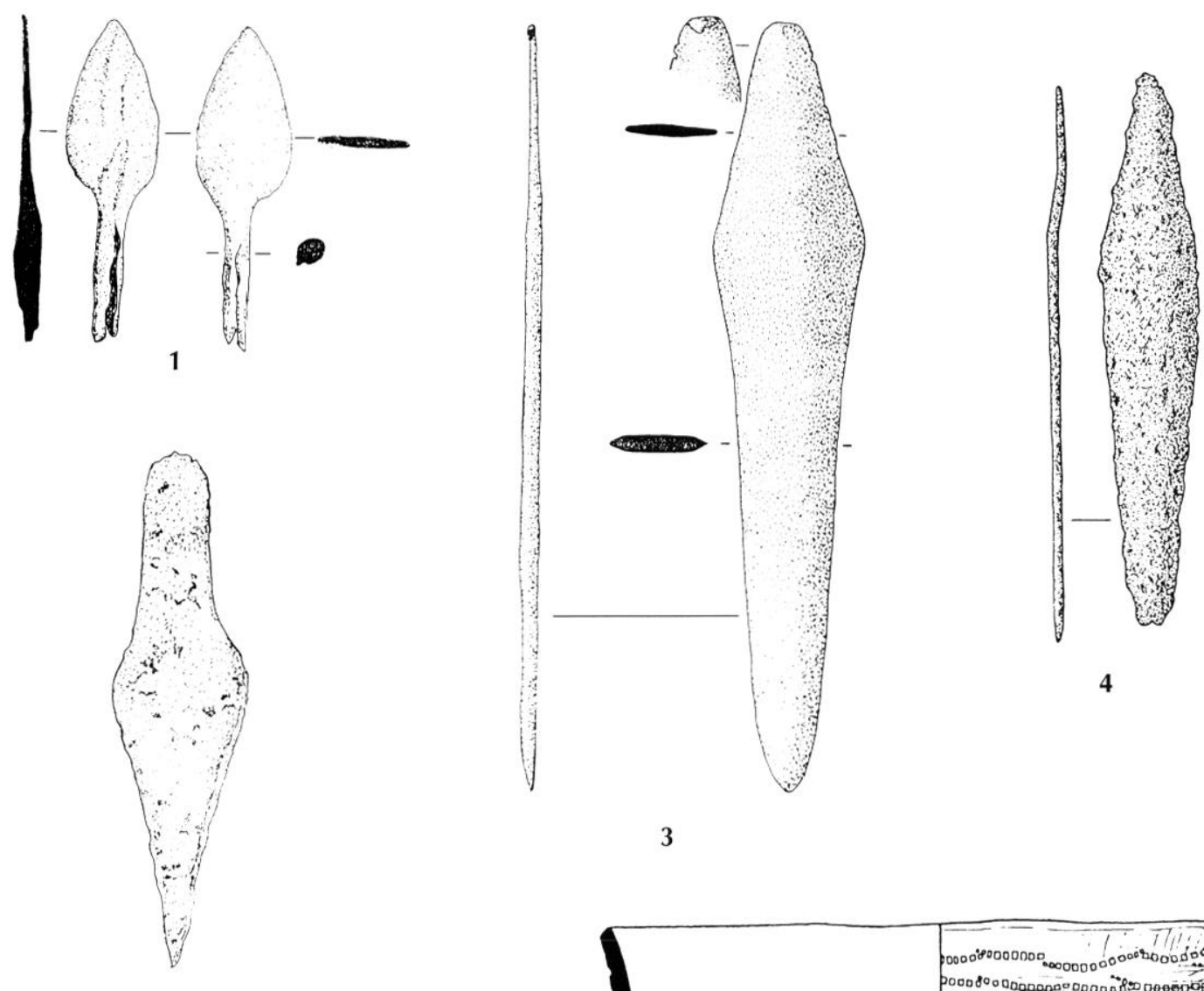

4

3

2

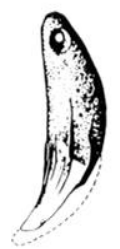

5

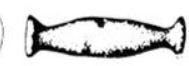

(1)

6

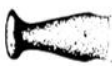

0

7

$5 \mathrm{~cm}$

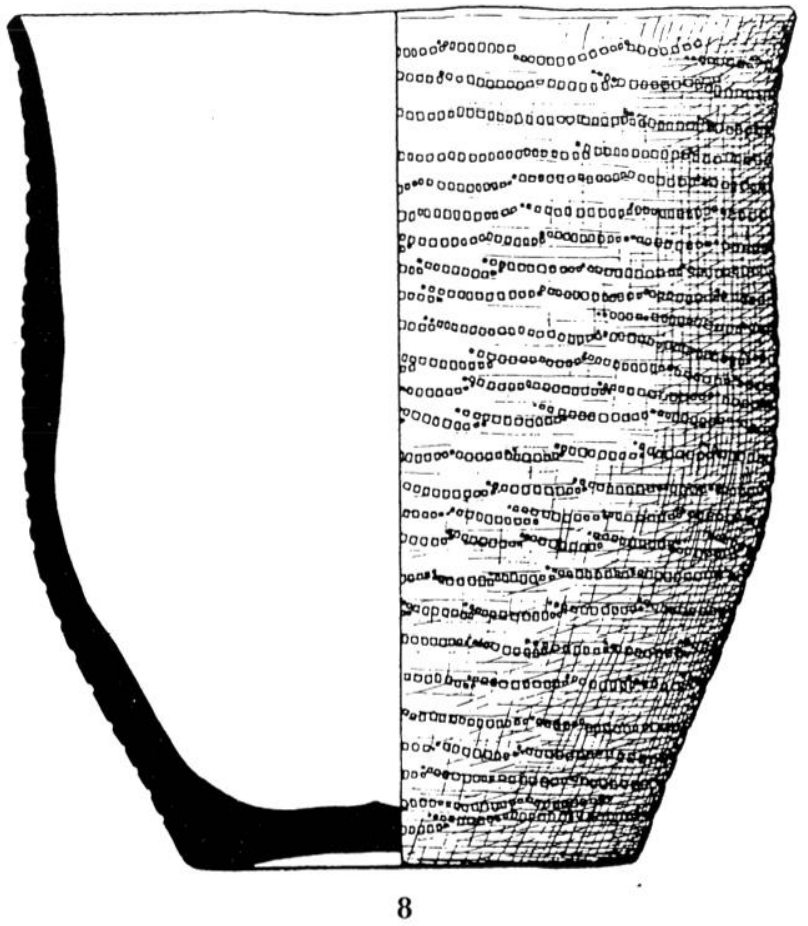

Fig. 60 - 1-4, mobilier métallique provenant de sites campaniformes de Sâ̂ne-et-Loire: 1, pointe de Palmella des Lacs à Epervans (dessin C. Michel); 2, poignard trouvé dans la Saône à Chalon-sur-Saône (dessin E. Eurard); 3, poignard tronvé dans la Sâ̂ne à Gergy (dessin A. Paglia); 4, poignard des Varennes à Dracy-le-Fort (dessin A. Paglia); 5-8: mobilier de la sépulture de Vertempierre à Chagny (dessin J.-P. Thévenot). 


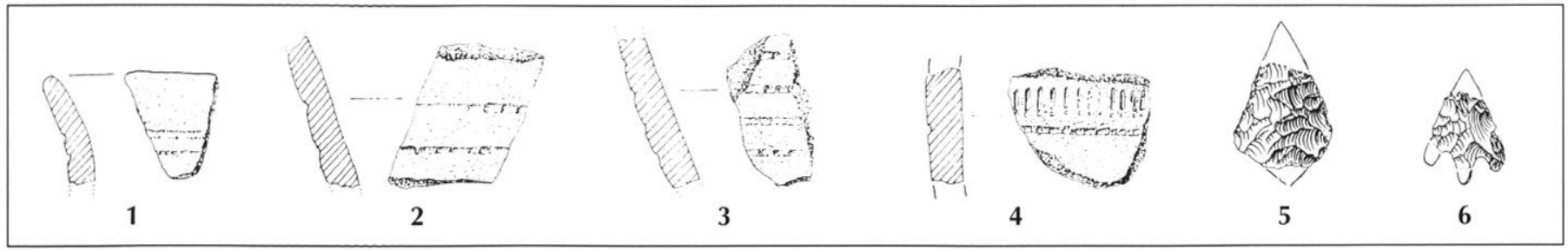

a

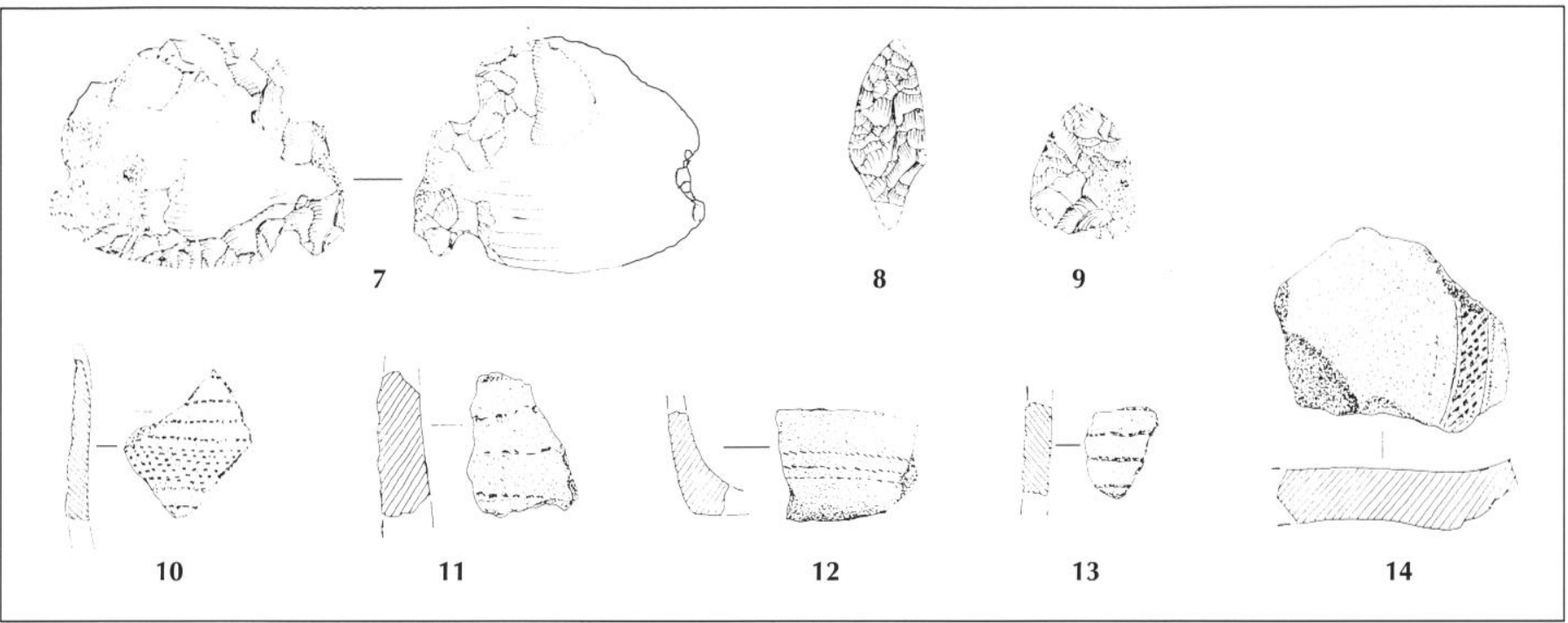

b

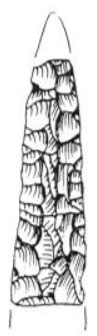

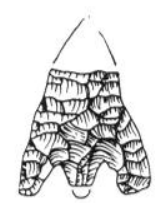

16

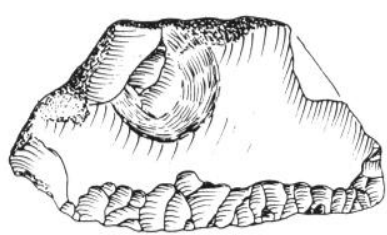

17
15
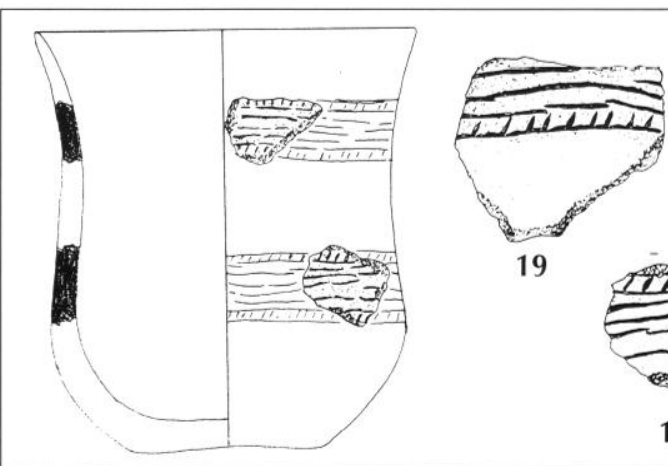

19

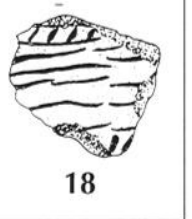

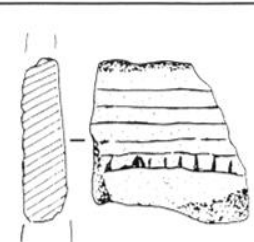

20

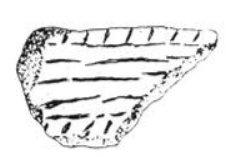

21

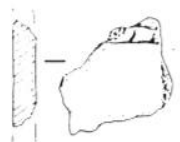

22

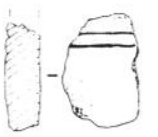

23 $(-\cdots)$

24

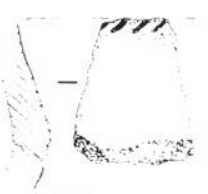

25

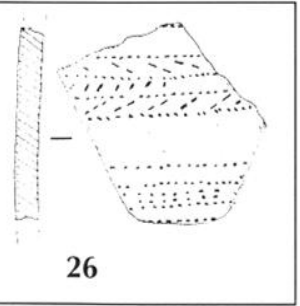

d
0 $5 \mathrm{~cm}$

Fig. 61 - Mobilier campaniforme provenant de Gergy et d'Ouroux-sur-Saône: a, Gergy' (1-3, 5-6, La Maladière; 4, La Prime) (dessin C. Michel); b, 7-14, Ouroux-sur-Saône, Carrière des Boulets (dessin C. Michel); c, 15-19, Ouroux-sur-Saône, Le Grand Bois et Le Taillis; d, Ouroux-surSaône, sans contexte (dessin A.-Ch. Gros). 
parfaitement identique à celui du Grand Bois (fig. 62). Plusieurs dizaines de tessons sont décorés, dans la majorité des cas au peigne. On remarque dans ce lot des fragments appartenant à un gobelet orné de bandes parallèles et de tracés verticaux, le tout exécuté très finement à l'aide d'un peigne à dents carrées. Le décor international et les impressions se retrouvent ici comme à Boitrait. Signalons également qu'il a été trouvé un petit fragment d'oxyde de cuivre qui pourrait être de la malachite selon les analyses réalisées.

Plottes (commune de Tournus), Beauvois. La prospection et la fouille de cette station de surface, implantée sur les collines du Tournugeois, à environ $4 \mathrm{~km}$ de la Saône, ont permis de détecter une occupation du Néolithique moyen qui a perduré dans une phase finale du Néolithique. On y a signalé un tesson orné d'un décor incisé en échelle (Duriaud, 1997b).

Saint-Georges-de-Reneins, Boitrait (Rhône). Très proche des limites du département de Saône-et-Loire, le site de Boitrait se trouve sur une éminence sablonneuse, à $1000 \mathrm{~m}$ environ de la Saône. Une fouille a permis d'observer qu'une couche unique contenait, avec une dizaine de microlithes géométriques, 13 armatures de types variés (tranchantes, à base droite ou concave, à pédoncule et ailerons). Parmi les tessons, un fragment de gobelet campaniforme est orné d'une bande d'incisions verticales et d'une bande d'impressions circulaires, séparées par une zone non décorée (fig. $58, \mathrm{n}^{\circ} 17$ ). Bien qu'à l'époque de la fouille, ce site ait été attribué au Tardenoisien III (Philibert, 1967), nous le considérons comme tout à fait caractéristique du Chalcolithique en val de Saône.

Saint-Marcel, Le Breuil. Ce site, découvert et étudié par L. Bonnamour, est certainement lié à un habitat de plein air, comme ceux des terrasses de la Saône (Bonnamour, Moine, 1963). À $600 \mathrm{~m}$ de l'habitat de La Noue et à $1200 \mathrm{~m} \mathrm{du}$ cours actuel de la rivière, il se trouve en terrain sablonneux, à $176 \mathrm{~m}$ d'altitude. L'industrie lithique est identique à celle découverte sur les sites d'Ouroux, Crissey et Gergy. La céramique comprend un bol à mamelon et des tessons d'un gobelet campaniforme orné de lignes horizontales effectuées par impression d'un peigne à dents carrées (fig. 59b).

Saint-Rémy, Le Moulin de Droux. Cet important habitat chasséen est situé lui aussi sur un monticule sablonneux (179 m d'altitude), en bordure de la petite rivière de la Corne et à $800 \mathrm{~m}$ du cours actuel de la Saône (site en cours de publication). Occupé dès le Mésolithique, il offre de rares vestiges chalcolithiques, cependant on peut rattacher au Campaniforme un minuscule fragment de vase décoré d'une ligne incisée à laquelle se raccordent des traits verticaux exécutés au peigne (fig. $58, \mathrm{n}^{\circ} 18$ ).

Tournus, La Croix Léonard. Bien que situé sur les collines du Tournugeois, ce site n'est qu'à 1500 m environ de la Saône. Les fouilles de J. Duriaud ont permis de retrouver les restes d'un foyer associé à une industrie lithique variée. La céramique comprend une trentaine de tessons campaniformes décorés par incisions de lignes, d'échelles et de chevrons emboîtés (Duriaud, 1997a; fig. 59c).

Varennes-le-Grand, La Maison Blanche. Il s'agit des restes d'un site des berges de la Saône qui n'a malheureusement pas fait l'objet de prospections régulières. A.-C. Gros y a recueilli, après une crue, quelques pièces sur la plage et dans la berge. Parmi celles-ci, figuraient un petit tesson campaniforme décoré de triangles imprimés au peigne, intercalés entre deux lignes parallèles, une armature tranchante, un grattoir et trois tessons de céramique commune décorés d'impressions inguiformes (fig. 58, n ${ }^{\text {os }} 9$ à 14). L'ensemble appartient de toute évidence au même niveau chalcolithique (Gros, Gros, 1968).

Vergisson, grotte des Tasnières. A. Jeannet a signalé un tesson campaniforme trouvé lors des fouilles de cette cavité, qui a été occupée à différentes époques (Jeannet, 1970).

\section{Les sites funéraires}

Bouze-lès-Beaune, Les Croconnets. Le site funéraire de Bouze-lès-Beaune, occupé du Néolithique au Bronze ancien, se caractérise par une nécropole tumulaire associée à deux alignements de sépultures en cistes. Cet ensemble funéraire jouxte un probable habitat où des tessons affleurent en surface du sol. Deux tumulus, situés au lieu-dit Les Croconnets (tumulus 3 et 4 ), ont livré du mobilier campaniforme. Dans le tumulus $n^{\circ} 3$, un gobelet décoré de lignes horizontales imprimées au peigne, deux armatures en silex, un fragment de lame, sept perles en calcite, un dentale, une canine de carnivore perforée et des fragments de grès, ont été découverts. Le tumulus $\mathrm{n}^{\circ} 4$ a livré des tessons campaniformes (sans autre précision) mêlés à des éléments lithiques et de la parure. Enfin, signalons que le tumulus n" 2 comportait cinq V-boutons (Baise, 1996).

Chagny, Vertempierre. Cette sépulture est située près de Chagny, à proximité du débouché de la Dheune dans la plaine de Saône. Fouillée par le docteur Variot en 1912, la publication a été réalisée par J.P. Thévenot (Thévenot, 1961). C'est la seule sépulture individuelle connue en Bourgogne à posséder un monument funéraire sous un probable tumulus. La sépulture comprenait un gobelet 


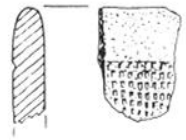

1

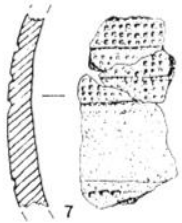

7

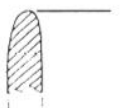

13

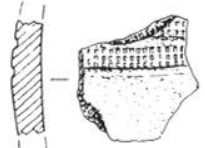

2

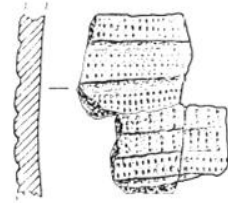

8

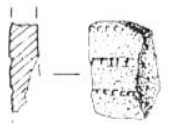

14

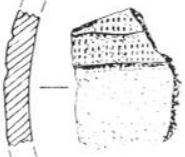

3
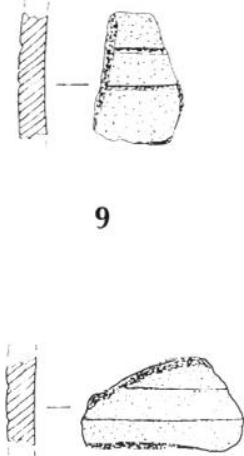

15

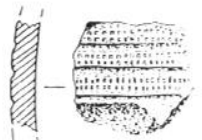

4

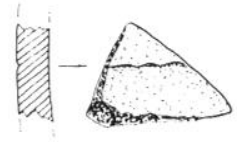

10

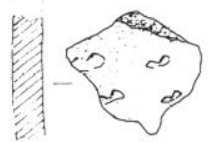

16

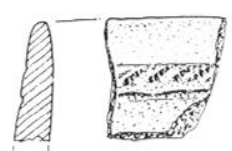

5

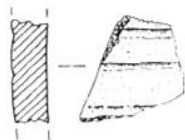

11

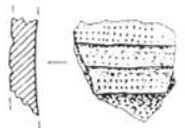

6

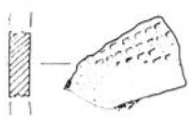

12

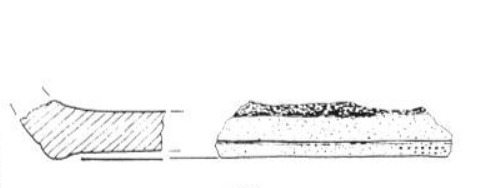

18

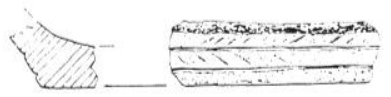

19

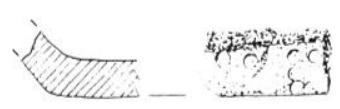

20

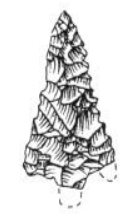

21

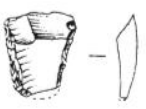

25

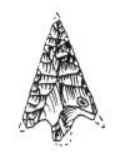

22

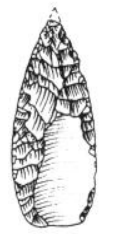

26

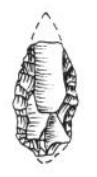

23

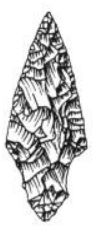

27

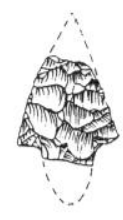

24

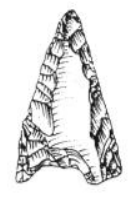

28

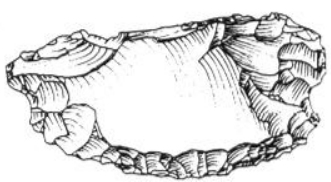

17

Fig. 62 - Mobilier campaniforme du site d'Ouroux-sur-Saône, Le Petit Bois: 1-16, 18-20, céramique (dessin C. Michel); 17, 21-30, mobilier lithique (dessin C. Bontemps).

décoré de lignes horizontales réalisées au peigne (Salanova, 2000). À proximité du vase, plusieurs éléments de parures en os ont été découverts (fig. $60, \mathrm{n}^{\text {os }} 5$ à 8 ).

Volnay, dolmen de La Pierre Brîlée ou La Grange au Vaget. Cette tombe mégalithique n'a livré qu'une pointe de flèche à pédoncule et ailerons équarris (Baise, 1996).

\section{Découvertes isolées}

Chalon-sur-Saône, la Saône. Un poignard en métal cuivreux, dit " en cuivre rouge " dans la publication d'origine (Cabrol, 1939), a été dragué dans la Saône "vers Chalon-sur-Saône " (fig. 60, n ${ }^{\circ}$ ). Ce poignard, d'un type caractéristique du Campaniforme, est très proche de celui mis à jour dans la tombe de Soyons en Ardèche (Blanc, 1958).

Gergy, la Saône. À la hauteur de Gergy, la drague a ramené du lit de la rivière un poignard losangique de $159 \mathrm{~mm}$ de long en métal cuivreux (non analysé), qui présente des traces de martelage et un tranchant nettement biseauté (Armand-Calliat, 1962; Bonnamour, 1969). Cette lame est comparable à celles découvertes en Languedoc (fig. 60, $\left.\mathrm{n}^{\circ} 3\right)$.

La Truchère, la Saône. Les dragages de la rivière ont anciennement ramené une tasse, décorée dans la partie 
supérieure de quatre bandes de trois lignes horizontales alternant avec des espaces remplis d'impressions circulaires concentriques, d'une ligne de chevrons, et d'un espace non décoré (Gaudron, 1956). L'ornementation de ce vase étant de type barbelé, il est à classer dans un Bronze ancien épicampaniforme (fig. 59d).

La carte de répartition des éléments campaniformes en Chalonnais montre le rôle essentiel de la Saône. Mis à part quelques incursions dans la Côte chalonnaise, comme Chassey, les habitats sont implantés à moins de $1000 \mathrm{~m}$ de son cours, dans une zone déjà occupée depuis le Mésolithique. L'analyse des décors, fossiles directeurs du Campaniforme, a depuis longtemps montré les influences du groupe rhénan. Cependant, certains éléments indiquent une influence méridionale, non seulement au niveau de la céramique (décor en échelle incisée proche des styles pyrénéen et provençal) mais aussi au niveau du mobilier métallique (pointe de Palmela d'Épervans, poignard de Chalon-sur-Saône proche des exemplaires de La Balance dans le Vaucluse et de Soyons en Ardèche).

O et A.-C. G.

\section{Le Campaniforme dans le Dijonnais}

\section{Les découvertes récentes}

En Bourgogne, le Dijonnais (Côte-d'Or) est la région qui a livré les plus nombreux témoignages sur le Chalcolithique ces dernières années. La plupart des sites se trouvent en général dans la périphérie immédiate de l'agglomération de Dijon. Ce phénomène est imputable à deux facteurs:

- l'intensification des travaux d'aménagement routiers et urbains dans ce secteur;

- la situation de Dijon au débouché de l'Ouche, voie naturelle vers la Seine et la Loire (fig. 63).

Il est raisonnable de penser que ces deux raisons se conjuguent pour faire de la région dijonnaise un secteur archéologique sensible, le dynamisme urbain actuel contribuant à une série de découvertes importantes.

\section{L'habitat}

L'habitat chalcolithique dans la région dijonnaise demeure mal connu. Quelques découvertes récentes et trouvailles inédites viennent éclairer la plaine dijonnaise et la vallée des Tilles. Elles ne concernent que des sites matérialisés par quelques rares structures, souvent noyées dans un plan contenant des structures protohistoriques plus récentes. Sur les plateaux calcaires de la Côte bourguignonne, l'habitat en grotte et la réoccupation de sites de hauteur sont les formes d'habitat les plus représentées (fig. 63). Les fouilles, en général anciennes, n'ont guère laissé de témoignages exploitables.

Quétigny, Les Allées Cavalières. Ce site a été découvert à l'occasion d'une campagne de diagnostic. Il se situe à l'est de l'agglomération dijonnaise, sur les pentes d'un coteau de faible hauteur dominant le ruisseau du Cromois, affluent de la Norge, qui alimente la vallée des Tilles et le bassin hydrographique de la Saône. Deux structures de type fosse ont livré un mobilier archéologique essentiellement composé de céramique (fig. $57, \mathrm{n}^{\text {os }} 1$ à 6 ). Ces structures se situent dans le périmètre d'un fossé, délimitant une zone ovoïde de $15 \mathrm{~m}$ sur $7 \mathrm{~m}$, et sont associées à des trous de poteaux très nombreux. Certains, situés près du fossé, semblent former les traces d'un bâtiment. Plusieurs autres structures comportant de la céramique datée du Hallstatt étant présentes dans le secteur, il nous est impossible d'évoquer une datation précise pour le fossé et le bâtiment qui ne contiennent aucun mobilier (Saint-Jean-Vitus, 2000).

Saint-Apollinaire, Sur le Petit Pré. Ce site d'habitat doit sa découverte aux travaux routiers de la déviation Arc-sur-Tille - Dijon. Il se localise au fond d'une petite vallée, près d'anciennes zones humides aujourd'hui comblées (Ducreux, Labeaune, 1999). Aucun cours d'eau ou ruisseau n'est associé à ce vallon. Le site est formé de structures de type trous de poteaux formant des alignements pouvant caractériser les traces de bâtiments. Le remplissage charbonneux des poteaux et les fragments de torchis contenus dans ces structures peuvent confirmer cette hypothèse. Les structures proprement dites sont recouvertes d'un niveau de faible épaisseur provenant vraisemblablement de l'altération du sol originel. Le mobilier associé à ce niveau est rare. Il se compose de céramique et d'industrie lithique datables du Chalcolithique, réparties dans plusieurs zones du site, ainsi que de céramique protohistorique plus récente. Le mobilier chalcolithique est surtout constitué de céramique non décorée, à cordon préoral (fig. 64a). Une analyse radiocarbone, réalisée sur un charbon de bois issu d'un trou de poteau (structure 7), a donné une date de 2467-2203 av. J.-C. (Ly 1884 [T. oz-1009] : $3875 \pm 40$ BP), associant cette structure à l'occupation chalcolithique.

Pluvet, Larrivoux. Situé dans la vallée des Tilles près de Genlis, le site de Pluvet a été occupé du Néolithique moyen jusqu'à la période gallo-romaine. L'occupation chalco- 


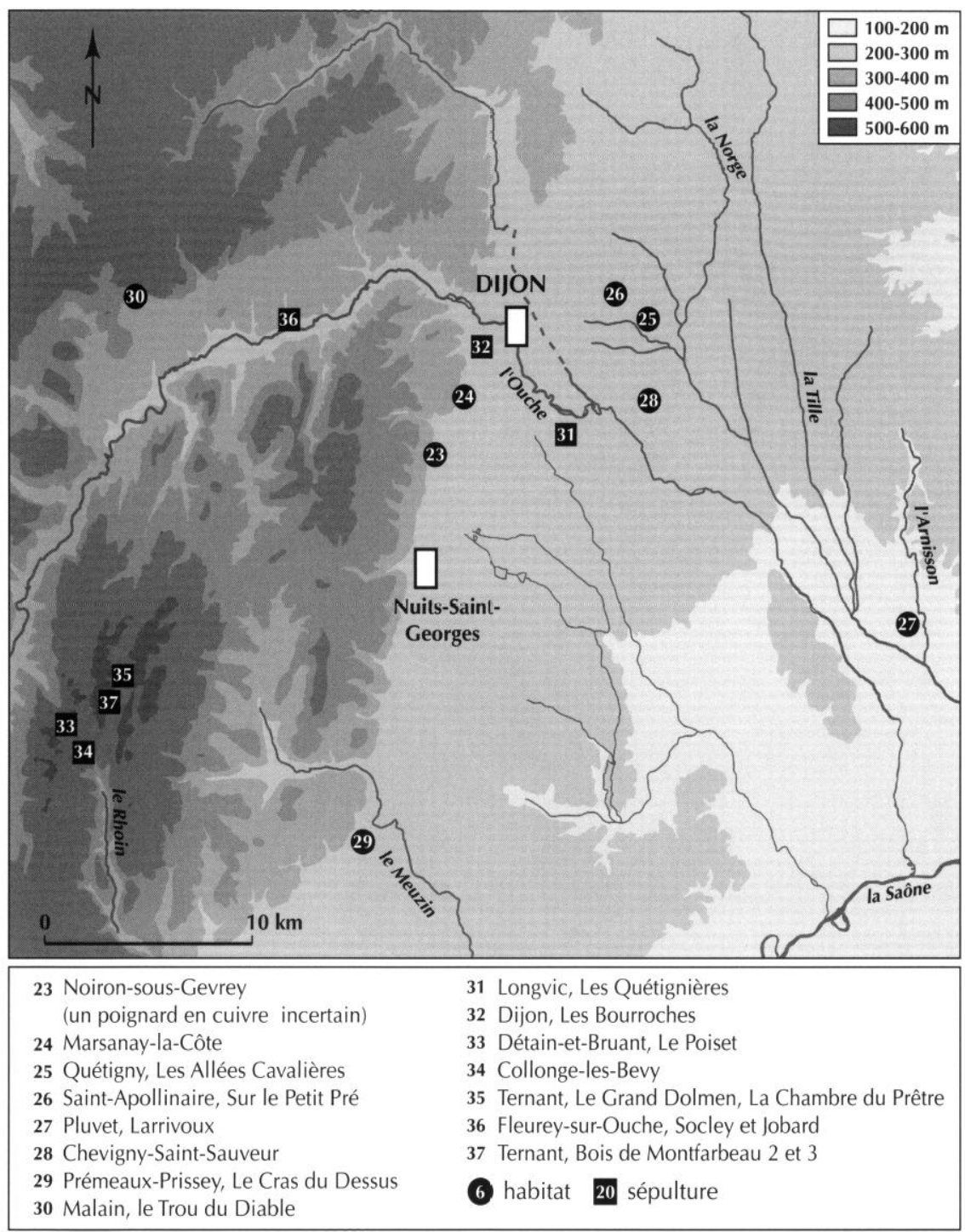

Fig. 63 - Carte du Campaniforme dans le Dijonnais (carte F. Ducreux).

lithique n'est pas très dense et se résume à quelques fosses relativement distantes les unes des autres. Les trous de poteaux, formant des bâtiments situés dans le même secteur, ne sont pas datables avec précision, puisqu'une occupation hallstattienne est attestée dans la même zone. Le mobilier livré par les fosses n'est pas très nombreux, mais bien conservé. Il s'agit pour l'essentiel de céramiques retrouvées écrasées sur le fond des fosses. Un vase à cordon préoral décoré sur la panse de coups d'ongle (fig. 64b) est comparable à un vase découvert à Saint-Aubin (Jura), associé à du mobilier campaniforme. L'industrie lithique est mal représentée et ne concerne que de très rares éclats de taille.

Chevigny-Saint-Sauveur [F. J. et Y. V.]. Ce gisement se situe au sud-est de l'agglomération dijonnaise, sur un terrain plutôt vallonné, ne disposant pas de cours d'eau. Les struc- tures ayant livré du mobilier chalcolithique sont plutôt rares, tout juste une ou deux (Virlogeux, 2002).

Le projet d'extension de la ZAC Excellence 2000 sur la commune de Chevigny-Saint-Sauveur (Côte-d'Or) concernait une surface d'environ 40 hectares. Préalablement aux travaux d'aménagement, une campagne d'évaluation s'est déroulée au cours de l'été 2000, suivie d'une fouille de sauvetage, de janvier à mai 2001. Le diagnostic, effectué sur l'ensemble de la surface concernée par le projet, a permis de mettre en évidence un ensemble de vestiges de natures et d'intérêts divers, allant de la fin de la période néolithique à l'époque moderne et s'étendant sur environ 15 hectares. L'intérêt principal du site s'est toutefois porté sur les périodes gauloise et gallo-romaine, pour lesquelles les témoins étaient plus nombreux et mieux structurés, 


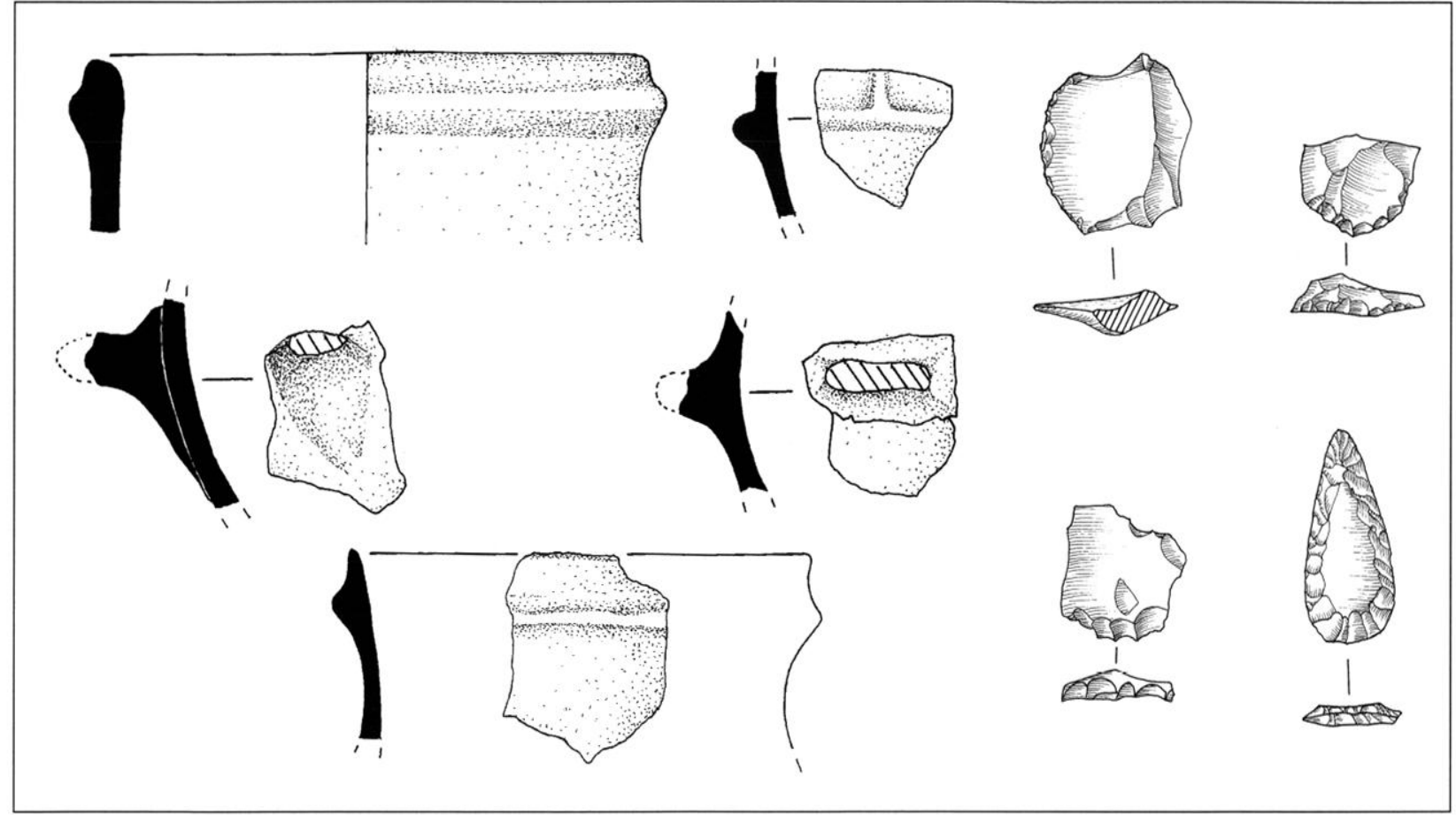

a

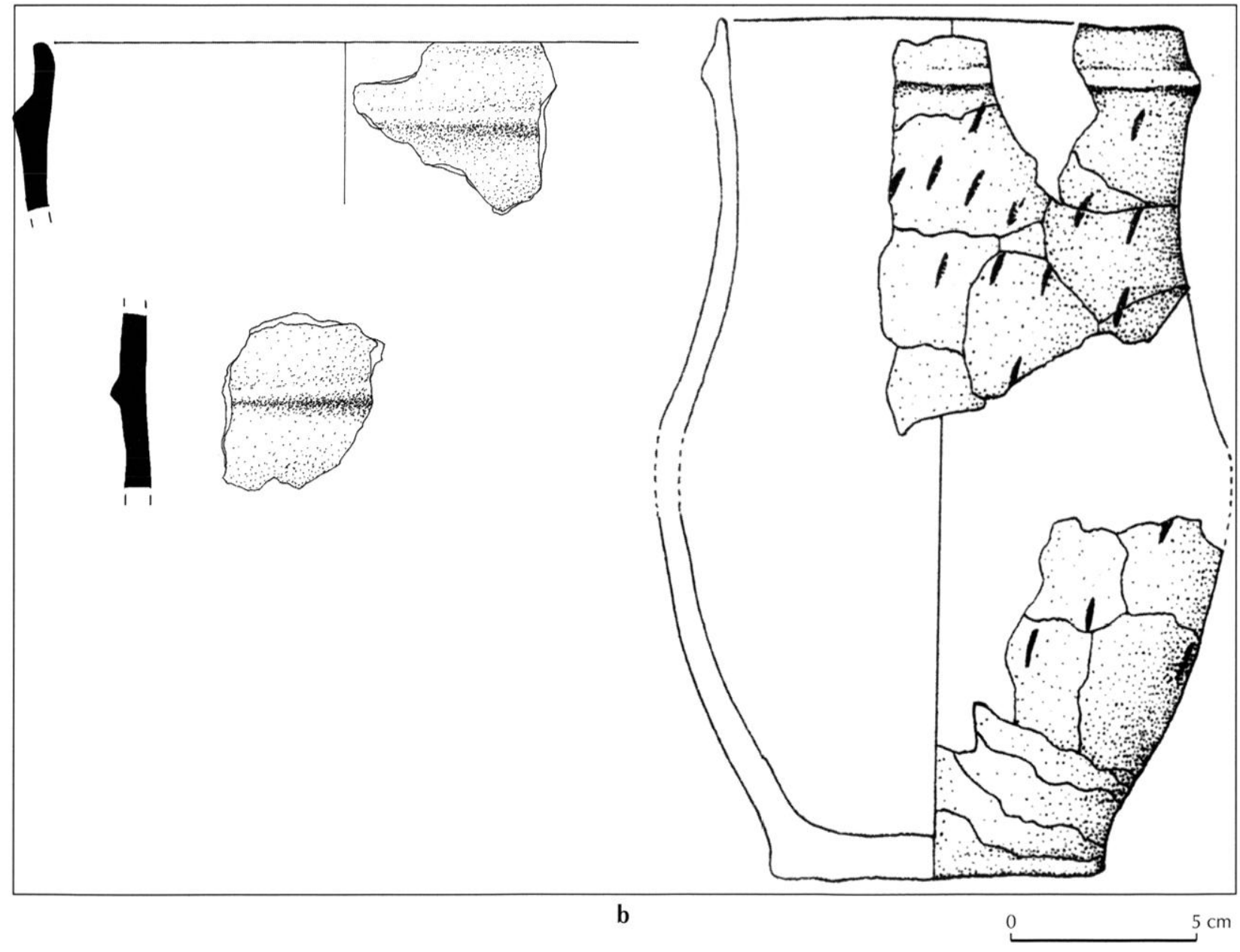

Fig. 64 - Mobilier campaniforme des sites: a, de Saint-Apollinaire, Le Petit Pré; b, de Pluvet, Larrivoux (dessin F. Ducreux, INRAP). 
permettant ainsi une étude plus approfondie. Les vestiges minoritaires, tel qu'un ensemble de structures chalcolithiques, n'en demeurent toutefois pas moins intéressants, et les découvertes de cette période sont encore trop peu nombreuses pour être négligées (fig. 65).

Seulement six structures $(2173,4507,4585,4586,4601$, 4610) ont été attribuées sans ambiguïté au Chalcolithique ${ }^{12}$.

La structure 2173, de plan ovalaire et aux parois subverticales, atteint une profondeur de $1,50 \mathrm{~m}$. Son remplissage stratifié est composé de plusieurs couches de teinte dominante grise. Un lot de tessons de céramique chalcolithique a été découvert à une cinquantaine de centimètres de profondeur, dans un niveau riche en charbons de bois. Bien que sa profondeur soit relativement faible, la forme de ce creusement ainsi que le faciès de son remplissage évoque plus un puits qu'un silo.

Les structures 4507 et 4610 , excavées en forme de cuvette, ont livré très peu de céramique. En revanche, les "fosses " 4585, 4586 et 4601 se sont révélées plus intéressantes. La présence d'une concentration de fragments de céramique et d'un tranchant de hache ont permis de mettre en évidence une occupation antérieure à un niveau gaulois présent sur plusieurs milliers de mètres carrés. Toutefois, le plan de la structure devinée n'a pu être relevé avec précision. Ainsi, la fosse 4586 n'a-t-elle été appréhendée nettement qu'en coupe. Il en a été de même pour la structure 4585 dont les limites exactes n'ont pu être observées. Il ne semblait s'agir que d'un amas de mobilier. Son plan est très approximatif. La structure 4601 est également apparue sous la couche gauloise. Elle se présente en plan sous la forme d'une vaste étendue avec des concentrations de charbons de bois en arc de cercle. Des limites nettes n'ont pas pu être cernées, même après plusieurs décapages successifs. Une tranchée nord-sud a donc été pratiquée pour essayer de percevoir si nous étions en présence d'un creusement ou non. Il semble s'agir d'une grande fosse incluant des parties sur-creusées.

Le mobilier recueilli se compose essentiellement de fragments de céramique. Mais les structures 2173, 4586 et 4585 ont également livré du matériel de mouture et la structure 4601, un tranchant de hache en roche verte.

La totalité du mobilier céramique représente 764 tessons pour un poids de $5,5 \mathrm{~kg}$ environ. Les structures 2173,4585

12. Malgré le faible nombre de structures décrites ici, la numérotation du Rapport final d'opération a été conservée afin d'éliminer tout risque de confusion. et 4601 ont fourni l'essentiel du matériel. La pâte de ces céramiques est de couleur orangée contenant en forte proportion des inclusions siliceuses (quartz, feldspath, mica). On peut observer à l'œil nu une grande variabilité dans la taille des cristaux contenus dans ce dégraissant. La présence de paillettes de mica en grande proportion est surtout remarquable dans la céramique recueillie dans les structures 4585,4586 et 4601 . L'épaisseur des parois (environ $1 \mathrm{~cm}$ ) ne varie guère d'un fragment à l'autre. La surface des récipients devait être régularisée, mais cela n'est observable que sur quelques fragments dont la surface n'a pas été altérée. Aucune restitution de profil complet ne peut être présentée car le remontage s'est avéré difficile. L'étude se base donc essentiellement sur les éléments remarquables, principalement du décor. On dénombre (fig. 65) :

- 5 fonds plats dont un présente, semble-t-il, une impression en forme de cupule;

- 2 bords non associés à un décor;

- 1 bord avec une perforation préorale;

- 1 bord avec une perforation préorale placée au-dessus de deux cordons lisses horizontaux;

- 1 bord avec un cordon horizontal nettement situé sous le rebord;

- 1 bord avec un cordon horizontal situé au sommet de la panse ;

- 1 bord avec un cordon horizontal nettement situé sous le rebord associé à des impressions à l'ongle ;

- de nombreux fragments de panse avec un décor d'impression à l'ongle disposé en lignes horizontales, devant appartenir à un unique exemplaire.

Ces éléments sont caractéristiques de la céramique dite d'accompagnement dont la typologie et la répartition se mettent en place depuis quelques années (Besse, 1996b, p. 165-180).

En effet les perforations préorales (Besse, 1996b, p. 169, type 7 ), les perforations préorales situées au-dessus d'un cordon lisse (Besse, 1996b, p. 169, type 8), un cordon horizontal nettement situé sous le rebord (Besse, 1996b, p. 169, type 5) et les décors à l'ongle (Besse, 1996b, p. 169, type 9) appartiennent au registre des principaux types de la céramique d'accompagnement campaniforme. On peut remarquer, dans la série de Chevigy-Saint-Sauveur, l'absence de gobelets décorés souvent associés à cette céramique.

Dans les régions de Bourgogne et de Franche-Comté, les récentes découvertes ayant livré de la céramique d'accompagnement campaniforme nous permettent certaines 


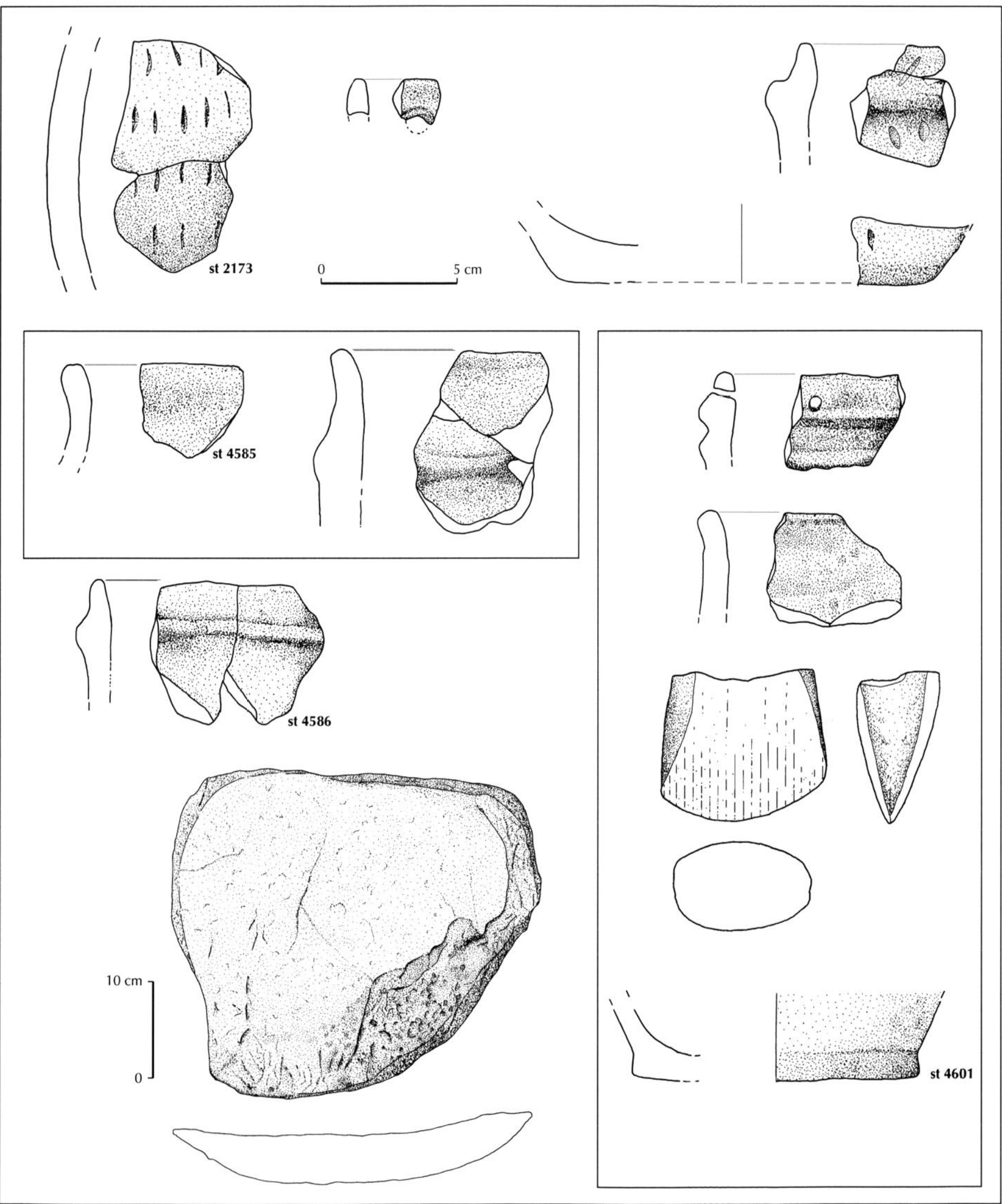

Fig. 65 - Mobilier campaniforme du site de Chevigny-Saint-Sauveur (dessin F. Jeudy). 
comparaisons. Ce sont les sites de La Noue à Saint-Marcel, (région chalonnaise), des Allées Cavalières à Quétigny, (région dijonnaise) et de À Daupharde à Ruffey-sur-Seille, (Jura bressan). Les vases avec un cordon horizontal nettement situé sous le rebord sont bien représentés, notamment sur les sites de Saint-Marcel et Ruffey-sur-Seille. Le décor d'impressions à l'ongle se trouve sur au moins un vase de Saint-Marcel et sur un autre vase provenant de Quétigny. Ce dernier permet aussi d'établir une comparaison avec le fragment de bord orné d'un cordon préoral surligné d'impression à l'ongle et souligné d'impression en cupule provenant de la structure 2173. Seul le site de Ruffeysur-Seille a livré des vases avec des perforations préorales situées ou non au-dessus d'un cordon horizontal. On peut cependant remarquer la particularité du fragment provenant de la structure 4601 avec la présence de deux cordons horizontaux situés sous une perforation.

L'absence de gobelet décoré, normalement associé à cette céramique d'accompagnement, ne permet pas une approche chronologique fine. Les éléments en notre possession nous fournissent une fourchette chronologique large, couvrant la phase finale du Campaniforme.

En ce qui concerne l'industrie lithique, un tranchant de hache en roche verte a été découvert associé à d'autres éléments mobiliers (structure 4585). La matière première étant très altérée, il est difficile de déterminer sa nature et sa provenance. Il s'agit d'un fragment de hache à section ovalaire dont les côtés sont piquetés. Il mesure $4 \mathrm{~cm}$ de largeur et $2 \mathrm{~cm}$ d'épaisseur au niveau de la cassure. La longueur conservée n'est que de $3,1 \mathrm{~cm}$. Le piquetage visible sur les bords témoigne d'un emmanchement dans une gaine, probablement en bois de cerf. La fracture se situerait donc au ras de cette gaine, comme on l'observe fréquemment. Une meule dormante entière a été découverte dans le remplissage de la structure 4586. Elle a subi une altération par le feu. La structure 4585 a également livré quelques portions de meules. La fosse 4601 a livré quelques éclats de boucharde. Deux fragments de polissoir (ou aiguisoir?) ont été recueillis dans la fosse 4586 . Notons enfin la présence d'un galet de quartzite portant des traces d'utilisation, dans le remplissage de la structure 4585 .

Les vestiges sont trop peu nombreux pour appréhender l'organisation de cette implantation néolithique. L'existence d'un habitat à cet endroit est toutefois plus que probable. La présence du puits est un indice fort. La céramique commune associée à des éléments de mouture corrobore cette hypothèse.

\section{Les sites funéraires}

La plupart des sites sépulcraux connus dans le Dijonnais sont des réutilisations de coffres mégalithiques datés du Néolithique moyen ou final. Les inhumations campaniformes viennent dans ce cas se superposer aux inhumations précédentes. Les fouilles réalisées sur ces sites étant la plupart du temps anciennes et peu documentées, il est difficile dans ces contextes de préciser si les inhumations campaniformes sont individuelles ou collectives. L'inhumation individuelle est en revanche attestée en plaine dijonnaise. Deux sépultures individuelles forment, avec la sépulture sous tumulus de Vertempierre à Chagny (Saône-et-Loire), un groupe de structures funéraires de premier ordre du fait de leur rareté.

Longvic, Les Quétignières. La sépulture des Quétignières à Longvic a été découverte très récemment, à l'occasion d'une fouille préliminaire à la construction d'un complexe industriel. Il s'agit d'une sépulture en fosse quadrangulaire de petites dimensions (1,3 $\mathrm{m}$ de longueur pour $0,45 \mathrm{~m}$ de largeur et $0,1 \mathrm{~m}$ de profondeur), comportant le squelette d'une femme âgée de 45 ans ou plus (étude anthropologique réalisée par L. Staniaszek, in Labeaune et al., 2003), déposée sur le côté droit avec les membres inférieurs fléchis (fig. 66). Le squelette est orienté nord-est - sud-ouest, la tête est au nord-est. La partie inférieure de la sépulture est recoupée par une réduction postérieure au niveau des jambes de la défunte. D'après l'étude anthropologique de la structure funéraire, le corps de la défunte était contenu dans une enveloppe souple. Le mobilier accompagnant cette sépulture est constitué d'un vase de type gobelet très érodé, de 4 coquilles provenant de bivalves de la famille des glycyméridés (détermination assurée par C. Bémilli, in Labeaune et al., 2003) percées par frottement au niveau du crochet de la coquille, et d'un éclat de silex de bonne qualité, vraisemblablement contenu dans les mains du sujet (fig. 66). Le mobilier est comparable à celui de la tombe des Bourroches à Dijon, notamment en ce qui concerne la parure.

F. D., F. J. et Y. V.

\section{Les découvertes anciennes ${ }^{13}$}

\section{L'habitat}

Auxonne, L'Abergement-les-Auxonne. Près de la Saône, un site a livré un poignard du Grand-Pressigny, des armatures

13. Les illustrations disponibles pour les sites qui suivent sont toutes regroupées dans l'ouvrage de J. Bill (1973). 


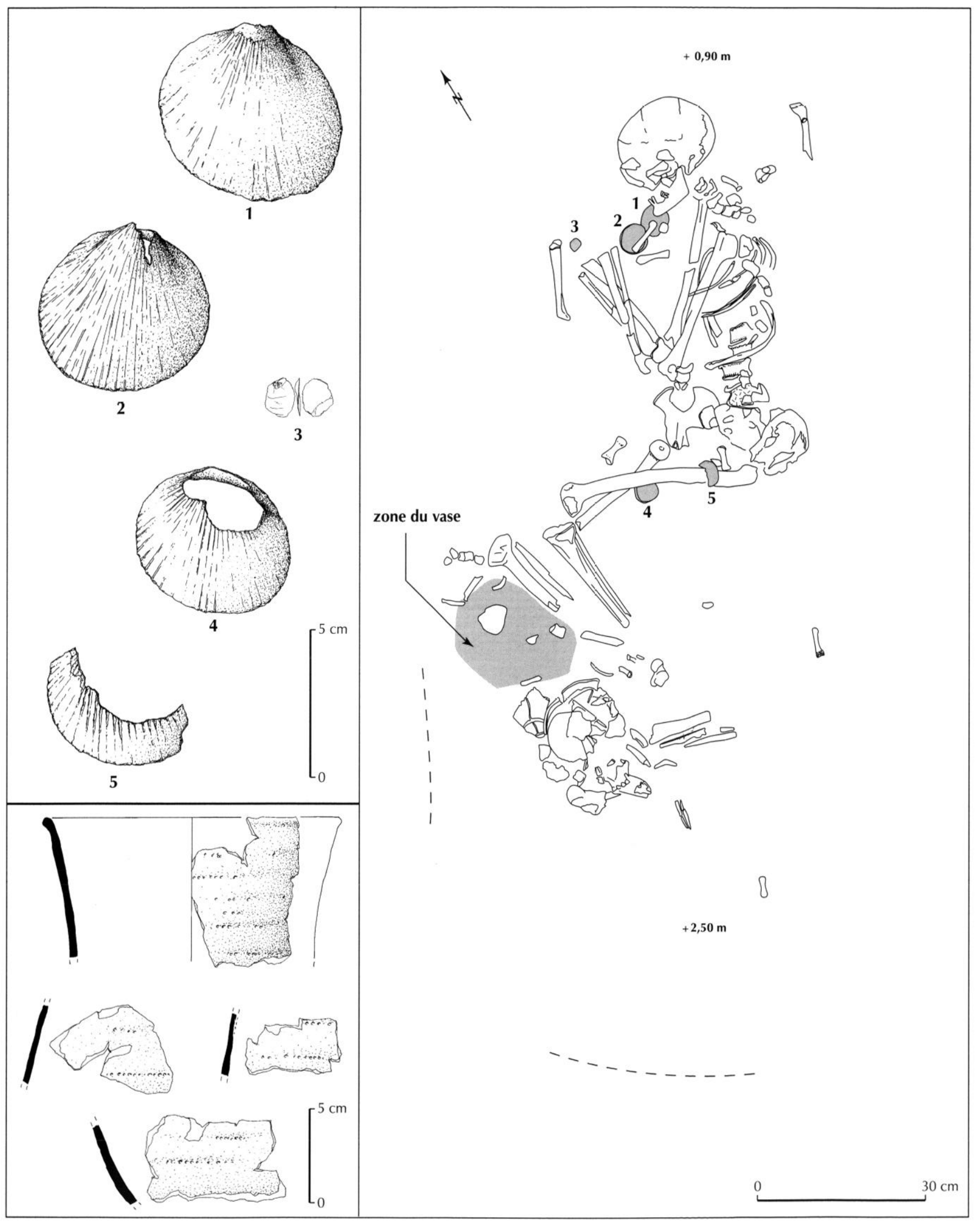

Fig. 66 - Plan et mobilier de la sépulture de Longvic, Les Quétignières (dessin F. Gauchet, INRAP). 
de flèche et quelques microlithes géométriques, ainsi qu'un tesson dont « la face extérieure est revêtue de deux séries de stries parallèles et irrégulières se rejoignant " (Bouillerot, 1932). La reproduction ne permet pas de déterminer la technique décorative employée sur ce tesson (impression au peigne ou à la cordelette).

Mâlain, Le Trou du Diable. Cette grotte, fouillée par P. Buvot, a livré un tesson campaniforme.

\section{Les sites funéraires}

Collonge-lès-Bévy. Ce coffre mégalithique renfermait deux V-boutons en os (Nicolardot, 1968).

Détain-et-Bruant, coffre mégalithique du Poiset. Cette sépulture mégalithique contenait 14 à 16 inhumations. Parmi celles-ci, un gobelet à décor de lignes horizontales imprimées au peigne et une pointe de flèche à pédoncule et ailerons ont été découverts (Guyot, 1940; Dorion et al., 1987).

Fleurey-sur-Ouche, tumulus $n^{\circ} 1$ de Socley. Cette sépulture, fouillée anciennement, a livré deux tessons campaniformes, l'un décoré de bandes hachurées décorées au peigne, l'autre orné de lignes horizontales imprimées à la cordelette (Nicolardot, 1968).

Fleurey-sur-Ouche, tumulus $n^{\circ} 9$ de Jobart. Un tumulus de la nécropole de Fleurey-sur-Ouche a livré un mobilier se rattachant au Campaniforme. Il s'agit d'un tesson campaniforme décoré d'une bande hachurée imprimée au peigne (Nicolardot, 1968).

Marcilly-Ogny, Champ du Saule 1 et 2. Le site de MarcignyOgny est localisé dans la région de l'Auxois, à plusieurs dizaines de kilomètres à l'ouest de Dijon. Deux sépultures ont livré chacune 19 et 121 tessons campaniformes. Dans le premier cas, ils correspondent à un gobelet campaniforme décoré de lignes horizontales, qui était associé à un V-bouton en tortue (Baise, 1996).

Prémeaux-Prissey, Cras du Dessus. Ce site, fouillé par le docteur Planson au début des années soixante-dix, a livré une petite fosse contenant 7 tessons campaniformes, correspondant à 2 vases: l'un orné de bandes de lignes imprimées au peigne, l'autre de lignes imprimées à la cordelette (Joly, 1968).

La Rochepot, La Chaume. Cette tombe mégalithique a livré des tessons campaniformes, sans autre précision (Joly, Puissegur, 1965).

La Rochepot, Les Épenottes. Cette tombe mégalithique a livré au moins 4 vases campaniformes fragmentés et une pointe de flèche à pédoncule et ailerons équarris (Joly,
Puissegur, 1965; Bill, 1973). L'un des tessons porte un décor incisé en échelle alors que les autres sont décorés au peigne de bandes hachurées, de lignes horizontales et de chevrons emboîtés.

La Rochepot, La Garenne. Cette tombe mégalithique a livré deux tessons campaniformes ornés de bandes hachurées imprimées au peigne (Joly, Puissegur, 1965; Bill, 1973).

Ternant, Le Grand Dolmen ou La Chambre du Prêtre. Cette sépulture mégalithique a livré quelques tessons de style campaniforme (Thévenot, 1978).

Ternant, tombes du Bois de Montfarbeau 2 et 3. Ces deux tombes mégalithiques ont livré respectivement 1 vase et 4 tessons campaniformes (Baise, 1996).

O. et A.-C. G., complété par F. D. et L. S.

\section{ÉTUDE DES SÉRIES DE COMPARAISONS}

Afin de ne pas créer de confusion avec les analyses de La Noue, la numérotation des groupes pétrographiques sera comprise entre I et $\mathbf{n}$.

\section{Site de la ZAC des Allées Cavalières à Quétigny (Côte-d'Or)}

Le site a livré 94 tessons, représentant $538 \mathrm{~g}$ de céramique. Le nombre d'individus est de 6 vases: 1 tesson décoré de bandes de croisillons, imprimées au peigne fin; 2 tessons à décor indéterminé; 3 tessons de panse imprimés au peigne très fin d'un décor de bandes hachurées contractées; 17 tessons d'un gobelet fin érodé, décoré de lignes réalisées par impression de peigne fin; 70 tessons appartenant à un vase à bord rentrant portant un cordon à section carrée, de $1,1 \mathrm{~cm}$ de large, décoré de chevrons incisés sur le cordon et, sur la panse, de traits verticaux incisés désorganisés (fig. 57).

Le gisement est localisé sur des terrains oligocènes, difficilement distinguables des épandages récents (Clair, 1982).

Les trois céramiques analysées ( $\mathrm{n}^{\text {os }} 46,47$ et 48 ) ont une matrice phylliteuse et cotonneuse. Les inclusions sont moyennement abondantes, émoussées et de taille comprise entre $20 \mu \mathrm{m}$ et $1,2 \mathrm{~mm}$. Le quartz est dominant. Le feldspath potassique et les micas sont toujours présents tandis que le plagioclase est rare. Les fragments de roches d'origine plutonique sont en quantité variable.

En fonction de la nature du cortège des autres inclusions, deux ensembles peuvent être distingués. 
Groupe I: à seuls éléments d'origine plutonique et métamorphique (vase $\mathrm{n}^{\circ} 46$ )

Les deux types de micas sont relativement abondants. Les lithoclastes d'origine plutonique sont moyennement abondants; ils sont constitués par l'association de minéraux quartzo-feldspathiques et micacés. Les fragments issus de roche d'origine métamorphique sont peu abondants; il s'agit de gneiss à biotite.

Groupe II: à éléments d'origine plutonique et sédimentaire (vases $n^{\text {os }} 47$ et 48 )

Le mica noir est abondant tandis que le mica blanc est plus rare. Les fragments de roches d'origine plutonique sont peu abondants, constitués comme le précédent groupe, de l'association de minéraux quartzo-feldspathiques et micacés. Quelques carbonates sont présents, plus abondants dans la pâte du vase $n^{\circ} 48$, constitués de fragments de sparites, de coquilles et de bioclastes indéterminables. Des éléments calcédonieux ou des silex sont également présents.

La pâte de ces deux vases est proche. L'origine de l'argile du vase $n^{\circ} 46$, décoré de croisillons, est à situer sur ou près d'un massif granito-gneissique à deux micas tandis que celle des individus $\mathrm{n}^{\circ} 47$ et 48 , respectivement décorés de lignes horizontales réalisées au peigne, se trouve en domaine sédimentaire carbonaté, mais comportant une alimentation homogène en éléments granitiques également à deux micas, pouvant d'ailleurs être identique à celle de l'autre groupe.

Les formations granitiques sont présentes à l'ouest (Morvan) et au sud-ouest (monts du Charollais), mais les plus proches se situent à l'est dans le massif de la Serre.

\section{Site de Larrivoux à Pluvet (Côte-d'Or)}

Le site de Larrivoux a livré 68 tessons, correspondant à un poids de $440 \mathrm{~g}$, et une boule d'argile (99 g). Trois vases peuvent être individualisés: 1 tesson de bord portant un cordon en " $V$ " de $0,8 \mathrm{~cm}$ de large, placé $2 \mathrm{~cm}$ sous le bord; 2 tessons d'un vase à cordon en " $U$ ", collé, de $0,7 \mathrm{~cm}$ de large (bord non conservé) ; 65 tessons d'un vase portant à cordon en " $U$ » modelé dans la masse de $0,6 \mathrm{~cm}$ de large, placé à $1,2 \mathrm{~cm}$ sous le bord, à fond plat et décoré d'impressions à l'ongle sur la panse (fig. 64b).

Ce gisement se situe au bord de la Tille, dans des formations alluviales quaternaires argilo-limoneuses (Clair, 1982).

Les deux individus céramiques analysés ( $n^{\circ} 49$ et 50) sont sensiblement différents même si leur matrice est phylliteuse et cotonneuse.
Groupe III: à éléments d'origine carbonatée, plutonique et métamorphique (vase $\mathrm{n}^{\circ} 49$ )

Seul le mica noir est présent. Il est abondant. Les inclusions sont moyennement abondantes, émoussées à usées et de taille essentiellement réduite $(<100 \mu \mathrm{m})$. Le quartz domine tandis que les deux types de plagioclases sont peu abondants. Les fragments de roches quartzofeldspathiques d'origine plutonique et métamorphique sont rares. Un des lithoclastes montre une structure granophyrique. Les carbonates, peu identifiables, sont relativement abondants.

Groupe IV: à seuls quartz et carbonates (vase $\mathrm{n}^{\circ} 50$ )

Les inclusions sont peu abondantes, émoussées à usées, de taille comprise entre $20 \mu \mathrm{m}$ et $1,2 \mathrm{~mm}$. Le quart $z$ domine le cortège formé également de quelques carbonates altérés par la cuisson.

L'origine de l'argile des deux vases se situe en domaine sédimentaire. La pâte du vase $\mathrm{n}^{\circ} 49$, décoré de coups d'ongle, renferme une fraction en éléments d'origine granito-gneissique à seul mica noir. Des origines identiques à celles des vases du groupe I peuvent être proposées. Le cortège minéralogique du vase $n^{\circ} 50$ portant un cordon est beaucoup plus réduit et la terre à l'origine de ce vase ne peut pas être située avec plus précision. Une origine locale est tout à fait envisageable pour ce vase.

Les deux vases renferment de la chamotte, en abondance pour le vase $n^{\circ} 49$ et en quantité plus réduite pour le $n^{\circ} 50$. Les micas noirs et les quartz sont abondants dans les grains de l'individu $n^{\circ} 49$, indiquant une possible origine identique des tessons broyés.

\section{Site de Corvée Rateau à Beaune (Côte-d'Or)}

Ce site a livré 9 tessons représentant $69 \mathrm{~g}$ de céramique. Le nombre d'individus est de 5 vases: 2 tessons appartenant à 2 vases décorés de lignes horizontales imprimées au peigne; 1 bord à cordon (section en " $U$ » collé, $1 \mathrm{~cm}$ de large) et portant des empreintes d'un outil bifide de part et d'autre du cordon; 1 tesson non décoré et 3 tessons appartenant à un fond plat (fig. 57).

Le site est localisé sur des terrains quaternaires alluviaux (Rémond et al., 1985).

Le seul individu céramique $\left(n^{\circ} 51\right)$, portant un cordon préoral, issu de ce site présente une matrice phylliteuse et cotonneuse. Les inclusions sont moyennement abondantes, émoussées à usées et de taille comprise entre $20 \mu \mathrm{m}$ et $3 \mathrm{~mm}$. Le quartz domine tandis que le plagioclase est peu 
représenté. Le mica noir est relativement abondant, tout comme les fragments de roches d'origine plutonique associant les différents minéraux.

L'origine de l'argile ne renfermant aucun élément d'origine sédimentaire est à rechercher dans un massif montagneux dans lequel affleure un granite à biotite. En effet, l'argile employée correspond à une altérite de ce type de roche. Vers l'ouest, les monts du Charollais renferment les plus proches granites à biotite, déjà rencontrés lors de l'étude du site de La Noue. Plus lointains, les granites du Morvan et de la Montagne de la Serre peuvent être aussi potentiellement envisagés.

Quelques grains de chamotte sont présents dans la pâte $\mathrm{du}$ vase. Ils renferment du quartz et du mica noir tout comme la matrice encaissante.

Tout comme pour La Noue, des terres issues du cristallin ont été employées pour la fabrication de quelques vases campaniformes ( $\mathrm{n}^{\text {os }} 46$ et 51 ) de la Côte-d'Or. Hormis peutêtre le site de Beaune, la distance de ces formations est plus importante que pour La Noue. Également, se retrouvent les argiles recueillies en domaine sédimentaire renfermant une fraction de cristallin ( ${ }^{\text {os }} 47,48$ et 49 ) qu'il est plus difficile de localiser faute d'éléments de comparaison, mais qui ne semblent pas affleurer dans le proche voisinage. Enfin, le dernier vase $\left(\mathrm{n}^{\circ} 50\right)$ a été probablement fabriqué avec une terre locale.

$$
\text { F. C. et L. S. }
$$

\section{La PLACE de La Noue}

\section{AU SEIN DU CAMPANIFORME BOURGUIGNON}

Le nombre de sites campaniformes découverts en Côted'Or et en Saône-et-Loire est à peu près égal. Cependant, dans l'un des départements le mobilier provient essentiellement de contextes funéraires (des sépultures mégalithiques fouillées anciennement), alors que dans l'autre il est issu de contextes domestiques, connus grâce aux prospections des berges de la Saône (tabl. XXIV).

\section{La céramique}

La série de La Noue a enrichi considérablement le corpus de Bourgogne orientale qui ne comptait jusque-là que 87 vases campaniformes (tabl. XXIV).

La comparaison de tous ces ensembles montre que La Noue s'insère parfaitement dans le répertoire régional (fig. 67). Les décors sont dominés par les panneaux de
Tabl. XXIV - Décompte du Campaniforme en Bourgogne orientale (hormis La Noue).

\begin{tabular}{|l|r|r|c|}
\hline \multicolumn{1}{|c|}{ Décompte } & $\mathbf{2 1}$ & $\mathbf{7 1}$ & Total \\
\hline Nb habitats & 7 & 16 & $\mathbf{2 3}$ \\
\hline Nb sépultures & 16 & 2 & $\mathbf{1 8}$ \\
\hline Nb déc. isolées & 2 & 3 & $\mathbf{5}$ \\
\hline Total Nb sites & $\mathbf{2 5}$ & $\mathbf{2 1}$ & $\mathbf{4 6}$ \\
\hline NMI vases & $\mathbf{5 3}$ & $\mathbf{3 4}$ & $\mathbf{8 7}$ \\
\hline
\end{tabular}

lignes horizontales et, dans une moindre mesure, par les bandes hachurées, les bandes d'échelles et les chevrons. Cette monotonie ornementale est caractéristique de la région qui développe un style propre et qui n'offre pas de corpus caractéristique du standard européen (Salanova, 2000). La Noue se distingue seulement par la présence (en petit nombre) de thèmes rares (triangles, croisillons) voire absents des autres sites (métopes, fermeture Éclair). Au niveau des techniques décoratives, l'homogénéité régionale est là encore surprenante: l'impression au peigne est quasi systématique. En revanche, les formes sont beaucoup plus diversifiées à La Noue et certains types de récipients n'existent que là (pichets et cruches, coupes polypodes).

Aucun cas d'association avec des vases du Néolithique final (ou Chalcolithique) local ni d'exemples de transferts stylistiques ou techniques ne peuvent être évoqués.

L. S.

\section{L'industrie lithique}

Le corpus lithique de La Noue est caractérisé par l'emploi de matières premières locales (silex du Secondaire et chaille) sur galets et sur petits rognons n'excédant pas la dizaine de centimètres.

À La Noue, la relative pauvreté des roches siliceuses employées et la monotonie des outillages recensés semble révéler un mode de gestion des matières et des artefacts autarcique et exempt de diffusion, d'échange et de contact avec les groupes campaniformes régionaux ou extrarégionaux. Pour preuve, les seules armatures de flèche identifiées (modèle triangulaire et trapézoïdal), d'obédience Néolithique moyen et final, sont encore fabriquées et utilisées à La Noue à la fin du III ${ }^{\mathrm{e}}$ millénaire. La perduration de ces modèles de flèche jusqu'au cours du Campaniforme, dans le Chalonnais semblerait témoigner d'une forme de syncrétisme avec les assemblages lithiques taillés de tradition locale. Ce syncrétisme se traduirait par la longévité de 


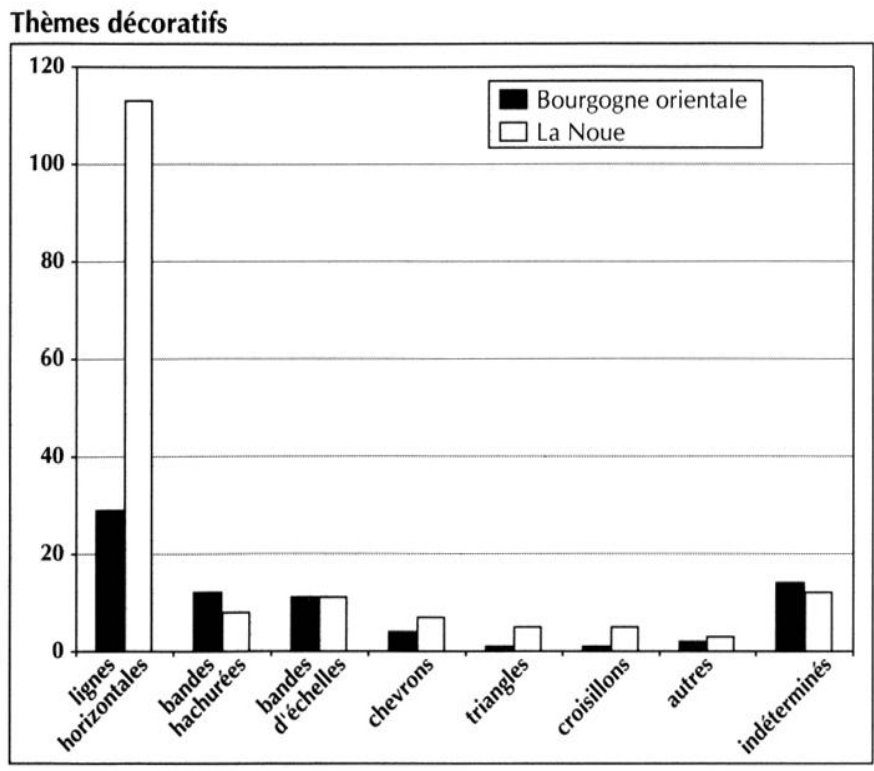

Techniques décoratives

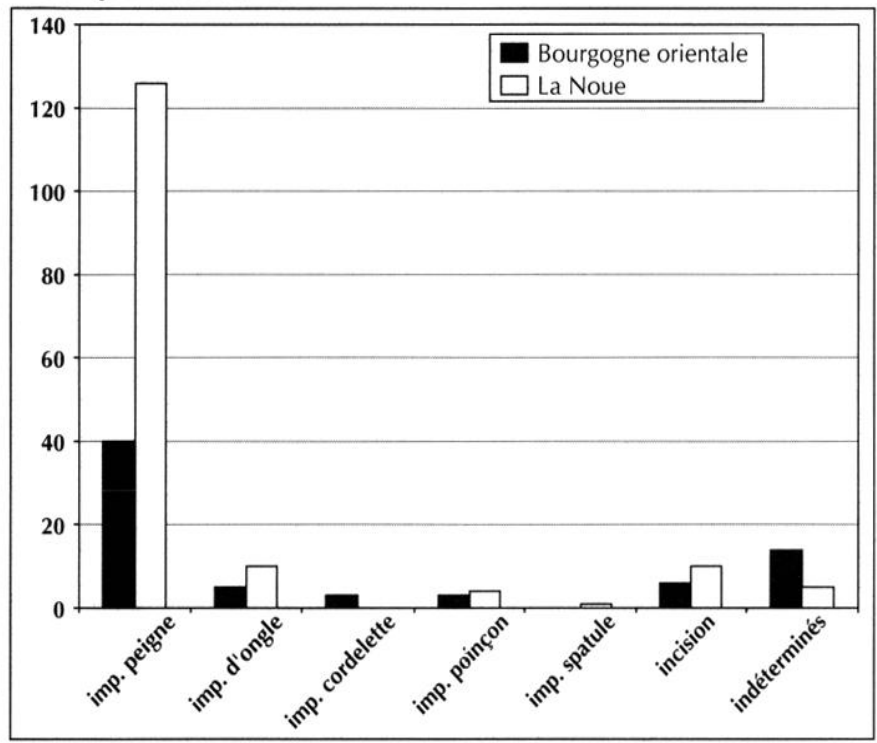

Formes des vases

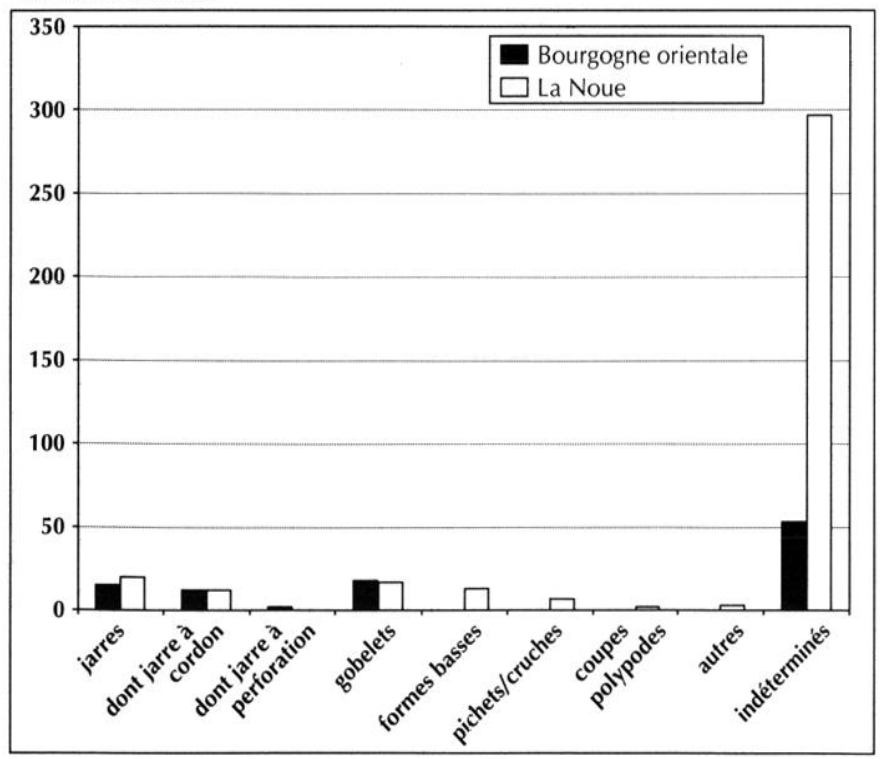

certains modèles typologiques et par des adoptions, voire plutôt des adaptations techniques, au cours du temps, d'un groupe culturel à l'autre.

Dans le même contexte du Campaniforme régional, on évoquera à titre de comparaison, l'exemple du site du Mont à Crissey, où un modèle d'armature de flèche triangulaire perçante, façonnée dans un silex traité thermiquement a été recensé. Ce modèle de flèche, probablement importé, est d'ailleurs associé à un débitage d'éclat et de lame produit à la percussion indirecte, au sein duquel certaines lames dépassent les $8 \mathrm{~cm}$ de long. Au Breuil, site localisé à $400 \mathrm{~m}$ environ de La Noue, les modèles d'armatures pédonculées à ailerons récurrents et les racloirs à encoches en silex exogènes sont présents parmi les outillages. Ces outils échangés sous forme de produits finis, dont plusieurs sont en silex du Grand-Pressigny, se retrouvent parmi les séries d'Ouroux-sur-Sâone, au lieu-dit Les Boulets. Ces quelques exemples soulignent la disparité typologique et culturelle entre la série de La Noue et d'autres séries régionales, même s'il s'agit d'échantillonnages issus de collections anciennes.

\section{S. S.}

\section{ÉLÉMENTS DE DÉFINITION DU GROUPE BOURGUIGNON-JURASSIEN}

\section{Historique DES RECHERCHES}

Dans les synthèses sur le Campaniforme français, les régions Bourgogne et Franche-Comté étaient généralement peu abordées du fait de l'indigence des données (groupe "rhéno-rhodanien " ou "Rhin-Rhône " [Riquet, Guilaine, Coffyn, 1963] «groupe du Rhône moyen » et du "CentreEst et de la Bourgogne" [Treinen, 1970]). Les influences centre-européennes et rhénanes, perceptibles dans le mobilier, avaient déjà été soulignées notamment avec l'abondance des décors de type Monsheim, c'est-à-dire des décors linéaires simples (Bill, 1973). A. Gallay a par la suite repris l'étude de cet ensemble, à partir du site de ChampVully (Gallay, 1986). C'est à lui que l'on doit l'appellation de " groupe bourguignon et jurassien ".

Suite à la découverte d'habitats campaniformes et de nombreuses céramiques communes associées aux vases campaniformes décorés, A. Gallay crée le complexe rhodano-

Fig. 67 - Comparaisons des séries céramiques de Bourgogne orientale et de La Noue. 
rhénan qu'il voit comme un développement autonome du Campaniforme dans la phase moyenne et récente de son évolution (Gallay, 1986). Ce complexe est essentiellement caractćrisć par des grandes jarres à profil en " $S$ ", munies de cordons préoraux et très rarement de décors imprimés à l'ongle (Gallay, 1986, p. 432). La céramique décorée est alors considérée comme du "matériel associé ". Au sein de ce complexe, A. Gallay distingue 5 groupes régionaux: le groupe rhénan, le groupe bourguignon et jurassien, le groupe de la France méridionale, le groupe atlantique et le groupe de l'Italie septentrionale. Depuis, les découvertes en contexte domestique se sont multipliées et les travaux de $\mathrm{M}$. Besse ont montré la très grande répartition en Europe de la céramique commune définissant à l'origine le complexe rhodano-rhćnan (Besse, 2003a, 2003b). La céramique commune ne suffit donc plus à définir une entité régionale, mais elle renvoie plus généralement à une identité culturelle adoptée sur une large échelle. Le terme de " complexe rhodano-rhénan " doit être abandonné puisque ses caractéristiques dépassent largement le cadre régional initialement défini (Bailly, Besse, 2004).

Au contraire, l'analyse de la céramique décorée permet de tracer des frontières au sein du vaste complexe campaniforme. Ainsi, c'est de la récurrence de certains décors céramiques que sont nés les groupes rhodano-provençal et pyrénéen. Dans le rcstc de la France, les décors céramiques campaniformes présentent une si forte variabilité ou au contraire une si grande monotonie qu'il est plus difficile de caractériser des ensembles régionaux. Néanmoins, l'étude des décors sur le site de Derrière le Château à Géovreissiat, et les comparaisons qui s'imposaient dans un contexte régional nous avaient permis d'esquisser les contours d'un ensemble stylistique cohérent, centré sur le Rhône et qu'il restait à définir (Salanova, 1997). Des connexions avec l'Italie centrale et la Provence avaient alors été mises en évidence.

Si la céramique décorée est un traceur efficacc, cllc ne saurait cependant suffire à définir ce groupe régional. En effet, nous disposons désormais d'un large spectre propre à sa culture matérielle, mais aussi de données socioéconomiques, certes encore embryonnaires, grâce à 3 habitats fouillés et étudiés récemment, bien conservés et relativement comparables au niveau de leur mobilier: Champ-Vully à Rances (Vaud, Suisse) (Gallay, Baudais, 1985; Besse dir., 2003), Noir Bois à Alle (Jura, Suisse) (Othenin-Girard, 1997) et Derrière le Château à

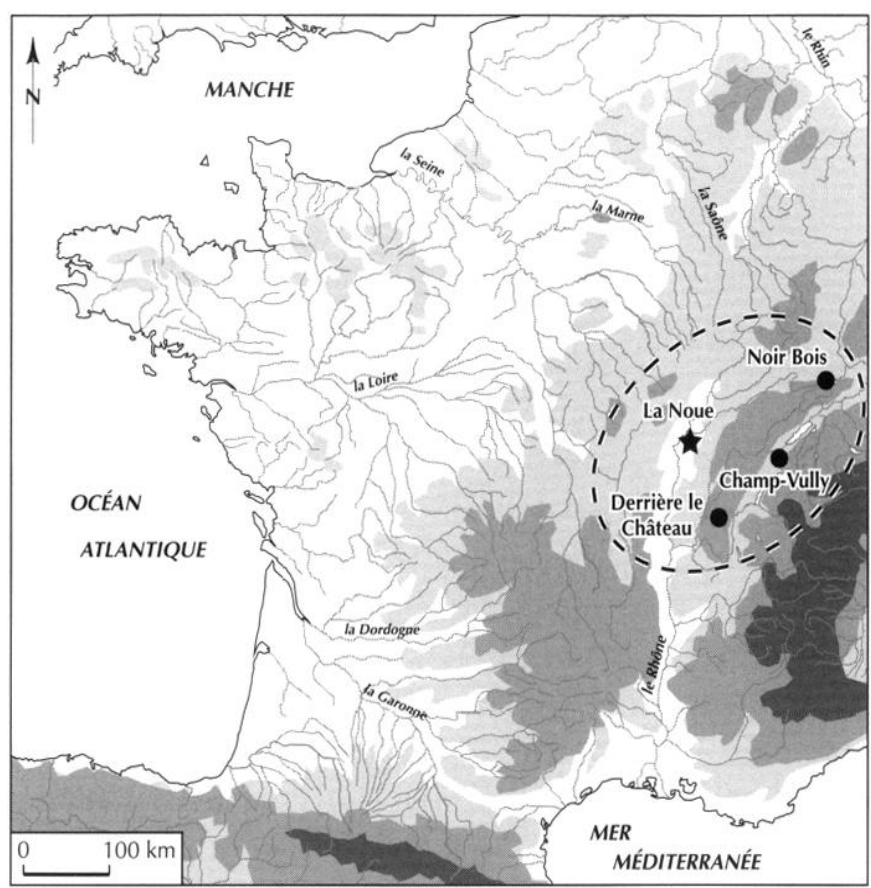

Fig. 68 - Localisation des trois sites de référence et du groupe campaniforme bourguignon-jurassien (carte L. Salanova).

Géovreissiat (Ain, France) (Salanova, 1997; Hénon, VérotBourrély, 1998; Besse, 2003). Avec le site de La Noue, ces trois sites représentent les ensembles domestiques les mieux connus pour le Campaniforme (fig. 68).

Nous baptiserons ce groupe "bourguignon-jurassien " en référence aux travaux déjà menés par A. Gallay (1986) qui avait tracé son contour géographique. Bien que peu esthétique, ce terme est préférable aux labels « RhinRhône " ou "rhodano-rhénan " qui renvoient explicitement à la céramique commune. Nous limiterons la discussion aux quatre habitats mentionnés ci-dessus, car ils fournissent des données variées mais contrôlables, dans la mesure où nous avons été impliqués dans leur étude. L'intégration de corpus funéraires fouillés anciennement ou de découvertes sans contexte précis, dont nous avons montré l'identité stylistique supra, nous semble inutile pour la démonstration. De même, la place de ce groupe dans un contexte chronologique plus large est pour le moment peu claire. En effet, le Néolithique final pré-campaniforme reste très mal connu dans l'est de la France, excepté dans les habitats des berges de lacs. Malheureusement, nous savons que ces habitats sont abandonnés avant ou au moment où le Campaniforme apparaît.

L. S. 


\section{APPROVISIONNEMENT EN MATIÈRES PREMIÈRES}

\section{Les matières premières siliceuses}

L'approvisionnement en matières premières siliceuses du site campaniforme de Champ-Vully-est à Rances (Vaud, Suisse) apparait comme un apport en roches majoritairement régional (Affolter, 2003; Bailly, 2003). Sur un total de 182 artefacts, $59 \%$ de silex régionaux, $16 \%$ de silex locaux, $7 \%$ de silex extra-régionaux et $18 \%$ de produits lithiques indéterminés ont été recensés. L'essentiel de l'industrie est fabriqué à partir des silex de la région de Bellegarde et de Seyssel $(41 \%)$, ce qui induit la quête et le transport de ces roches siliceuses, le long d'axes nord-sud, sur des distances atteignant au moins une centaine de kilomètres, de la zone d'approvisionnement au site de Champ-Vully-est.

À Géovreissiat, l'occupation campaniforme du site de Derrière le Château a livré un total de 1037 artefacts, comptant 501 outils et 536 produits de débitage, les nucléus étant inclus dans ce décompte (Bailly, 2002a). $68 \%$ du total du débitage et $45 \%$ du total de l'outillage sont des silex du Crétacé, dont l'approvisionnement correspond à un potentiel de sources variées, localisées à plus de $5 \mathrm{~km}$ du site. Si l'on tient compte du panel des ressources siliceuses recensées, force est de constater la prépondérance de silex d'origine locale et régionale à Géovreissiat. Deux outils en silex du Grand-Pressigny appartiennent peut-être à ce corpus, mais leur contexte archéologique de découverte ne permet pas de les intégrer (Bailly, 2002a, p. 169).

Le site de Noir Bois à Alle (Jura, Suisse) a livré un corpus de 1165 artefacts, dont 189 outils et 976 produits de débitage, y compris les nucléus, ont été dénombrés (Detrey, 1997). $63 \%$ du corpus des artefacts sont produits en silex du Kimméridgien local et la majeure partie des roches siliceuses importées provient de Devellier (32\%), qui se situe également dans le canton du Jura suisse. Il s'agit d'un silex marin en position secondaire, qui est considéré comme un approvisionnement exogène, situé à plus de $10 \mathrm{~km}$ en terrain plat, ou à moins de $5 \mathrm{~km}$ en terrain accidenté depuis le site. Les outils importés sur de longues distances, c'est-à-dire de plus d'une cinquantaine de kilomètres, sont plus rares et ne totalisent que $3 \%$ du total de l'industrie (Affolter, 1997).

De tels transports et circulations des matières ne sont effectivement pas constatés à La Noue, puisque la recherche des silex, en relation ou non avec les réseaux d'approvisionnement des argiles, n'excède pas la vingtaine de kilomètres depuis la périphérie septentrionale du site. Aucun silex pressignien n'apparaît parmi la série lithique du Chalonnais, ni d'ailleurs aucun autre silex importé ou échangé à plus d'une centaine de kilomètres du site. Ce constat plaide en faveur d'une autonomie des occupants campaniformes de La Noue, en ce qui concerne l'approvisionnement en roches siliceuses.

Cette apparente « autonomie " du groupe de La Noue peut toutefois être nuancée par l'emploi de techniques variées, tant celles qui servent à produire les supports de taille que celles qui sont utilisées pour le façonnage des outils. Les connaissances techniques requises, plus ou moins complexes et élaborées, qui nécessitent des investissements marqués à divers degrés, ne peuvent à l'évidence être le fait de tailleurs isolés, privés de contacts avec d'autres spécialistes. Les savoir-faire mis en pratique sur le site sont ici multiples et il semblerait donc imprudent d'expliquer que les connaissances techniques soient exclusivement transmises de génération en génération et d'imaginer que les artisans tailleurs de La Noue soient dénués de tout contact avec d'autres spécialistes. De plus, la récurrence entre la série lithique étudiée et entre la plupart des industries campaniformes, éloignées géographiquement et caractérisées par l'emploi majoritaire (mais pas exclusif!) de matière première locale ainsi que par la prédominance des mêmes types d'outils, constitue un "dénominateur commun culturel " où la diffusion des idées, des techniques et des échanges des savoir-faire et des biens sont de mise entre groupes sédentarisés et itinérants à la fin du III $^{e}$ millénaire. $\mathrm{Si}$ les occupants du site ne semblent pas avoir pratiqué une économie d'échange à partir de certains produits lithiques (armature, poignard, lame exogène...), ces mêmes occupants et probablement certains spécialistes ont peutêtre entretenu des contacts avec d'autres groupes campaniformes en terme d'échange d'idées et de connaissances techniques.

De tels mécanismes de diffusion ont été mis en évidence. à partir de la circulation des vases campaniformes (Salanova, 2000, p. 179), où l'adoption de modes et de styles céramiques identifiée dans certaines régions diffère dans d'autres contextes où des cas de transferts techniques, à partir de contacts avec des populations étrangères, ont été observés. Ce modèle interprétatif peut s'appliquer à la série lithique taillée de La Noue, en rapport avec le transfert des techniques de taille et des besoins en outillages requis, trait culturel caractéristique des groupes campaniformes de même fonds commun; la part de la tradition locale parmi 
les principales composantes de l'industrie lithique étant toutefois toujours présente à La Noue.

Sur la base des 301 silex recensés, la production " autarcique " du groupe campaniforme de La Noue apparaît comme prépondérante par rapport à une économie d'échange, qui caractérise, par exemple, le site campaniforme de Champ-Vully à Rances, en Suisse occidentale, de même obédience culturelle. L'approvisionnement régional ou sub-local en roche dure des corpus taillés des sites de Géovreissiat et d'Alle, est comparable à celui qui a été observé à La Noue, à la différence près que, sur les deux premiers sites, quelques silex, même s'ils sont rares, proviennent d'origines lointaines, induisant des contacts avec des groupes campaniformes éloignés.

S. S.

\section{Les matières premières argileuses}

Dans l'est de la France et en Suisse occidentale, quelques sites campaniformes de plein air ont déjà fait l'objet d'analyses pétrographiques en lames minces. Il s'agit, en France, du site de Derrière le Château (Géovreissiat et Montréal-laCluse, Ain) (Convertini, 1997b) et, en Suisse, des sites de Noir Bois (Alle, canton du Jura) (Convertini, 1997a; Convertini, Othenin-Girard, 1997) et de (hamp-Vully-est (Rances, canton de Vaud) (Convertini, 2003). Hormis Noir Bois, les deux autres gisements ont également fait l'objet d'une étude par L. Salanova.

Même s'il existe parfois des cas particuliers, la céramique décorée et commune a été fabriquée avec des argiles similaires dans chaque site. Au sein des terres exploitées, il existe toujours une production exogène minoritaire associée à une composante locale dominante. À Noir Bois, un groupe de vases décorés a été fabriqué pour $75 \%$ d'entre eux dans une zone distante d'une cinquantaine de kilomètres. Sur le site de Derrière le Château, ce sont uniquement des vases décorés qui ont été fabriqués dans une zone relativement éloignée. Quant à Champ-Vully est, faute d'échantillons argileux de référence, il n'est pas possible de déterminer la part des vases exogènes, mais elle existe probablement. Les sites de La Noue, des Allées Cavalières et de Corvée Rateau, avec une fraction de vases étrangers à l'environnement local, montrent la même tendance que les sites du Jura. Néanmoins, contrairement à Noir Bois et à Derrière le Château, les céramiques de La Noue fabriquées avec les argiles les plus éloignées correspondent aussi bien à des vases décorés qu'à des récipients communs. Il semble donc que le statut de ces vases soit différent de celui des vases exogènes présents dans les sites jurassiens.

À Champ-Vully-est, une forte corrélation entre certaines argiles (groupes $\mathrm{A}$ et $\mathrm{B}$ ) et des types de motif décoratif (respectivement décor à la spatule ou à l'ongle et lignes horizontales au peigne) a été mise en évidence. Ce type de situation n'a pas été rencontré dans les deux autres sites, ni à La Noue.

La pâte des céramiques de tous les sites renferme des grains de chamotte, mais leur introduction n'est jamais systématique. En effet, selon les gisements, c'est un pourcentage qui peut varier entre $46 \%$ (Champ-Vully-est) et $95 \%$ (Noir Bois). La Noue, avec $52 \%$ de céramiques chamottées, est proche du site de Champ-Vully. On constate que ces particules ont été indifféremment introduites dans la céramique décorée et commune. Sur les sites de Derrière le Château et de Champ-Vully, aucune relation n'apparaît entre présence ou absence de chamotte et type d'argiles, pas plus qu'avec la nature de l'ornementation ou le morphotype. À Noir Bois, la chamotte est quasi-exclusive et les deux vases n'en renfermant pas sont décorés au peigne et, surtout, ont une origine exogène. Ce constat a pu être élargi à la majorité des vases étrangers recueillis sur le site grâce à une étude exhaustive de la céramique à la loupe binoculaire. Sur le site de La Noue, quelques cas de vases du même type, fabriqués avec des argiles locales, peuvent montrer de fortes affinités avec la chamotte, mais également l'inverse.

Seule la céramique de Derrière le Château renferme des carbonates anguleux ajoutés dans l'argile. Ces particules sont abondamment présentes dans les pâtes des récipients des cultures du sud de la France. La situation géographique et les antécédents culturels de cette zone jurassienne sont probablement à l'origine de cette pratique. En effet, le sud du massif du Jura, mais également la partie médiane, ont souvent fait partie de la sphère d'influence méridionale (Convertini, 2001) tandis que la partie jurassienne septentrionale, le plateau suisse et la Bourgogne n'en font pas partie. D'ailleurs, Derrière le Château et I a Noue ont livré quelques céramiques décorées similaires à celles qui sont fabriquées sur les sites campaniformes du sud-est de la France. L'analyse n'a pas révélé une origine méridionale des argiles employées mais l'utilisation de terres locales ou régionales. Ces productions de type méridional ont donc été confectionnées dans le Jura et en Bourgogne ce qui implique des relations directes ou indirectes entre des groupes géographiquement très éloignés. Néanmoins, des productions étrangères attestées, témoins de déplacements 
Thèmes décoratifs

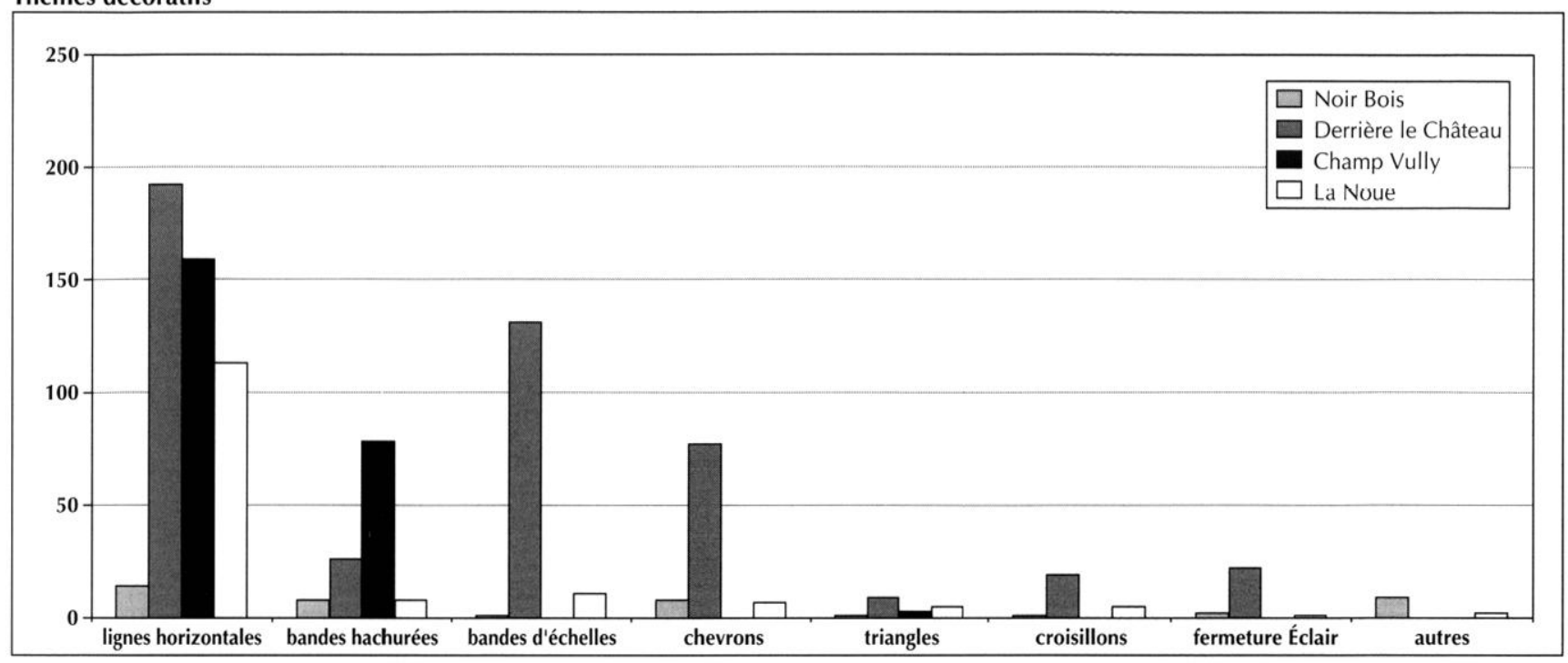

Techniques décoratives
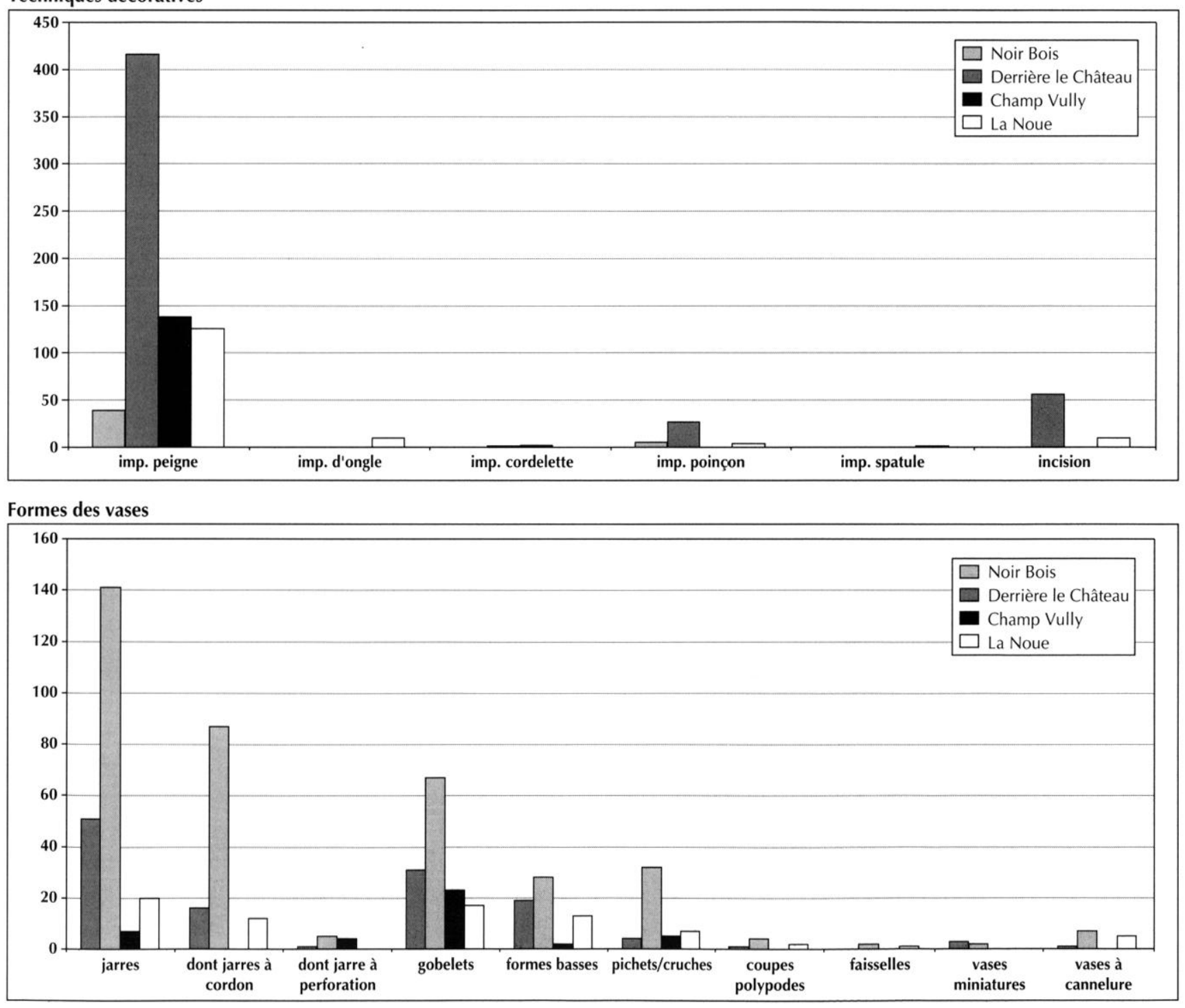

Fig. 69 - Comparaison des corpus céramiques des sites de Noir Bois, Derrière le Château, Champ-Vully et La Noue. 
et d'échanges, sont parvenues sur chacun des sites, même si l'on ignore encore par quels biais.

F. C.

\section{LE MOBILIER}

\section{Composition des batteries de cuisine}

La composition des spectres céramiques des quatre sites de référence peut faire l'objet de comparaisons, même si les effectifs ne sont pas équivalents (tabl. XXV): la série de Derrière le Château est de loin la plus fournie, mais également la moins homogène ${ }^{14}$.

$\mathrm{Au}$ niveau des décors, le répertoire des quatre sites est dominé par les lignes horizontales (fig. 69). Les bandes hachurées apparaissent en nombre significatif uniquement à Champ-Vully. Les thèmes en échelle, en chevron et en croisillon ne sont bien représentés qu'à Derrière le Château. Les "fermetures Éclair " sont présentes à Noir Bois et à La Noue, mais c'est à Derrière le Château que ce thème est le plus fréquent. Enfin, à Noir Bois, l'agencement des décors se distingue de celui des autres sites par la relative fréquence des éléments verticaux dans le remplissage des bandes.

Au niveau des techniques décoratives, le peigne est majoritaire sur les quatre sites. L'impression de cordelette n'est attcstéc avec certitude qu'à Champ-Vully, mais en faible nombre. Sur ce site, les peignes utilisés pour le tracé des décors ont des dents plus fines (Salanova, 2003).

$\mathrm{Au}$ niveau des formes, les jarres et les gobelets sont les plus répandus. Les jarres à perforations préorales, absentes de La Noue, sont présentes sur les autres sites mais en faible quantité. Seule la série de Noir Bois comprend des jarres à cordon digité. Les formes basses (bol, écuelle, jatte), les vases à cannelure et les coupes polypodes apparaissent en faible nombre sur tous les sites, sauf à Champ-Vully. Enfin, les faisselles et les vases miniatures sont anecdotiques dans les sćries de Noir Bois, Derrière le Château et La Noue.

Plusieurs de ces attributs céramiques évoquent des affinités culturelles extra-régionales. En ce qui concerne les décors, le quatuor classique se compose de lignes horizontales, échelles, bandes hachurées et chevrons. Cette monotonie des décors, déjà soulignée pour la Bourgogne,

14. La série céramique de Derrière le Château comporte en effet un pourcentage non négligeable de vases du Bronze ancien dont plusieurs vases à décor barbelé (Salanova, 1997; Besse, 2003a). Nous avons naturellement écarté ces vases des décomptes.
Tabl. XXV - Effectifs céramiques des sites de référence. du groupe bourguignon-jurassien.

\begin{tabular}{|l|r|}
\hline \multicolumn{1}{|c|}{ Site } & Nombre de tessons \\
\hline Noir Bois & 6146 \\
\hline Derrière le Château & 50400 \\
\hline Champ-Vully & 2586 \\
\hline La Noue & $\mathbf{2 5 9 2}$ \\
\hline
\end{tabular}

est également caractéristique du Campaniforme jurassien. Certains thèmes renvoient cependant à des décors centreeuropéens: les métopes à La Noue et l'agencement vertical des remplissages de bandes à Noir Bois (Othenin-Girard, 1997). La série de Derrière le Château comprend, quant à elle, des décors typiquement méridionaux: fonds rayonnants incisés, décors incisés-estampés en "fermeture Éclair » (Salanova, 1997). L'unique exemplaire de «fermeture Éclair » de La Noue pourrait témoigner d'une influence similaire. Ce type de décor est cependant fréquent en Bavière et dans le sud-ouest de l'Allemagne (Strahm éd., 1995). Si plusieurs éléments bourguignons, céramiques et métalliques, sont rapprochés d'exemplaires du sud de la France, à La Noue cette influence reste peu sensible.

En ce qui concerne les formes, les travaux de M. Besse nous apprennent que les cruches et pichets, les vases à anse horizontale et les coupes polypodes sont caractéristiques de l'Europe centrale (Besse, 2003a, 2003b). Les vases à cannelure sont au contraire rattachés au domaine du nord de l'Europe continentale (Pays-Bas, nord de l'Allemagne et nord de la France).

Le classement des sites de référence, selon leurs degrés d'affinités avec les différentes influences culturelles, montre que le site de Champ-Vully se distingue des trois autres sites (tabl. XXVI). Son assemblage ne renvoie qu'au fonds commun européen du Campaniforme et est certainement plus ancien que celui des trois autres sites. La Noue et Noir Bois ont en commun une forte influence d'Europe centrale, tant au niveau des formes que des décors. Cette influence est également perceptible dans les formes de Derrière le Château mais, sur ce site, les influences méridionales sont clairement attestées au niveau d'un petit lot de vases décorés.

D'une façon générale, l'assemblage céramique de La Noue est à classer dans un ensemble intermédiaire entre celui de Noir Bois, dont les décors sont plus proches de ceux d'Europe centrale, et celui de Derrière le Château, dont certains décors renvoient au monde méditerranéen.

L. S. 
Tabl. XXVI - Cilassification culturelle des siles de référence du groupe bourguignon-jurassien. (1) décor classique pour la région: lignes horizontales, bandes hachurées ou remplies d'échelles, cheorons; (2) thèmes supplémentaires: triangles ou croisillons; (3) formes d'Europe centrale: pichets et cruches, coupe polypodes, vases à anse horizontale; (4) décor d'Europe centrale: métopes ou remplissage vertical des bandes; (5) décor méridional: décor incisé-estampé, fond rayonnant, thème en fermeture Éclair. $X$, présence; $X X$, présence en grand nombre.

\begin{tabular}{|l|c|c|c|c|c|}
\hline \multicolumn{1}{|c|}{ Site } & $\begin{array}{c}\text { Décor } \\
\text { classique (1) }\end{array}$ & $\begin{array}{c}\text { Thèmes } \\
\text { supplémentaires (2) }\end{array}$ & $\begin{array}{c}\text { Formes d'Europe } \\
\text { centrale (3) }\end{array}$ & $\begin{array}{c}\text { Décor d'Europe } \\
\text { centrale (4) }\end{array}$ & $\begin{array}{c}\text { Décor } \\
\text { méridional (5) }\end{array}$ \\
\hline Champ-Vullly & $X X$ & $X$ & $X$ & & \\
\hline Noir Bois & $X X$ & $X$ & $X X$ & $X X$ & \\
\hline La Noue & $X X$ & $X X$ & $X X$ & $X$ & $X$ \\
\hline Derrière le Château & $X X$ & $X X$ & $X X$ & & $X X$ \\
\hline
\end{tabular}

\section{Les caractéristiques techniques et typologiques lithiques}

Si à La Noue deux chaînes opératoires ont été observées, l'une avec nucléus et l'autre sans, en particulier dans les locus $2,4,5$ et 6 pour la production de supports corticaux, les techniques de tailles sont variées puisque la percussion directe, indirecte et plus exceptionnellement, la pression, ont été employées. Un seul nucléus a été préchauffé avant la taille, ce qui pourrait être le fait d'un spécialiste, s'agissant là d'un cas isolé. Les produits de taille et les supports sélectionnés pour le façonnage des outils présentent une large palette de modules, de l'éclat à la fine lamelle à la lame courte. L'outillage est marqué par l'absence d'éléments microlithiques, des micro-denticulés et par celle de pièces foliacées, fréquents en plus ou moins grandc proportion parmi les séries campaniformes de référence (Detrey 1997, 2003; Bailly, 2002b; Billard et al., 1991; Furestier, 2002). Les produits de débitages recensés ne comptent pas non plus d'éléments d'amincissement ni d'éclats tangentiels, confirmant l'absence de pièces à retouches bifaciales sur le site. Ceci, à l'exception de deux éclats corticaux issus du locus 2, qui pourraient s'inscrire dans une chaîne opératoire de produits d'épannelage de pièce foliacée. L'emploi opportuniste d'éclats de première venue semble avoir été pratiqué occasionnellement, mais ceci ne peut être vérifić qu'à partir d'analyses tracéologiques complémentaires.

De même que pour la plupart des séries campaniformes, les grattoirs $(n=32)$ et les pièces esquillées $(n=9)$ sont majoritaires au sein de l'outillage de La Noue, par contre les racloirs à encoches et les armatures de flèches pédonculées à ailerons font défaut dans le corpus étudié. Ceci pouvant correspondre à une composante culturelle propre aux campaniformes de La Noue et à une sélection des outillages spécialisés en fonction des activités réalisées au sein des maisonnées. Une corrélation peut être ainsi faite entre l'absence de matière première exogène, d'outil importé, et d'armature de flèche pédonculée sur le site, en contexte d'habitat.

À Champ-Vully (Ranccs, Vaud, Suisse), sur 37 outils recensés, les grattoirs $(n=12)$ et les armatures de flèche $(\mathrm{n}=5)$ prédominent, puis viennent les pièces esquillées $(n=4)$ et les burins $(n=3)$. Seul un racloir double appartient à cette série. Les pièces à enlèvements marginaux $(n=10)$ et à enlèvements latéraux irréguliers $(n=3)$ ne sont pas comprises dans ce décompte, car il s'agit d'outils de moindre signification culturelle. À noter que les supports sélectionnés pour la confection des outils de Champ-Vully est sont rarement corticaux et qu'aucun d'entre eux n'est façonné sur éclat d'entame (Bailly, 2003).

Parmi les 501 outils du corpus de Derrière le Château (Ain), les grattoirs $(n=82)$ et les pièces esquillées $(n=46)$ prédominent. Viennent ensuite les outils à coches et les pièces denticulées $(n=30)$, les armatures de flèche $(n=22)$, les pièces bifaciales $(n=9)$ et les racloirs $(n=8) .144$ outils à enlèvements marginaux ont été recensés. Les grattoirs regroupent une grande variété de modèle, mais seuls trois d'entre eux sont obtenus sur lame. Les outils du site de Derrière le Château, peu investis techniquement, de même qu'à La Noue, sont généralement obtenus sur éclat $(n=119)$ et $45,8 \%$ d'entre ces outils sont fabriqués à partir de produits de plein débitage (Bailly, 2002b).

Sur le gisement de Noir Bois à Alle (Jura, Suisse), ce sont les pièces esquillées $(n=100)$ qui prévalent sur un total de 189 outils. Viennent ensuite les grattoirs $(n=18)$, les pièces à denticulation fine $(n=9)$, les racloirs $(n=8)$, les encoches $(n=7)$, puis les armatures de flèche et les troncatures qui comptent 5 exemplaires chacune. 25 éclats à retouches irrégulières ont également été recensés (Detrey, 2002).

Proportionnellement à ces trois sites de référence, la série de La Noue s'insère tout à fait parmi l'ordre d'apparition décroissant des outils, à ceci près que sur le site du 
Chalonnais, les armatures de flèches ne comptent que deux exemplaires. Le seul distal de lame de poignard recensé à $\mathrm{La}$ Noue s'apparente à un modèle également fragmenté, identifié sur le site de Combe en Vaillard à Chenevez (Detrey, 2002, p. 194, fig. 13, $\mathrm{n}^{\circ} 16$ ).

La prédominance des grattoirs et des pièces esquillées parmi les outillages du groupe campaniforme bourguignonjurassien souligne les caractères récurrents des industries lithiques, d'un site à l'autre. Sur certains gisements où les proportions s'inversent, les pièces esquillées sont majoritaires par rapport aux grattoirs, comme à Noir Bois et à Combe en Vaillard, en Suisse occidentale (Detrey, 1997).

Bien que les grattoirs prennent une part importante parmi les séries lithiques de la fin du IIIe millénaire (Billard et al., 1991 ; Furestier, 2002; Fillion, 2002), le manque de standardisation des supports est constant. Si la récurrence des grattoirs parmi les industries campaniformes est peutêtre plus la marque d'activités identiques qu'un simple phénomène typologique (Detrey, 2003, p. 199), ce constat peut, par extension, également s'appliquer aux cas des pièces esquillées. Ces derniers outils peuvent effectivement être fabriqués à partir de supports non standardisés, ou peuvent correspondre à des nucléus ou à des grattoirs réemployés.

\section{S. S.}

\section{LES ARCHITECTURES ET L'ORGANISATION DES SITES}

La partie étudiée du site de La Noue affiche une organisation spatiale dans laquelle les unités d'habitation prennent la forme de surfaces quadrangulaires disposées les unes derrière les autres, sur au moins trois rangées orientées nord-sud.

L'orientation et l'architecture des bâtiments semblent répondre à une adaptation au milieu naturel, certainement parallèle à la berge d'un bras secondaire ou mort de la Saône prolongeant l'actuel lac des Orlans. Associée à ces secteurs habités, une zone externe dispose d'une organisation caractérisée par des structures en creux et un récipient enterré. L'architecture proposée est celle de plates-formes édifiées sur des cadres de bois à divisions internes sur lesquelles un plancher est disposé. L'ossature des bâtiments est ensuite édifiée sur cette base par assemblage de type tenons - mortaises.

Si les formes d'habitat demeurent mal connues en Bourgogne de la fin du Néolithique au Bronze ancien, quelques exemples de sites récemment fouillés dans le domaine alpin et jurassien laissent entrevoir des comparaisons satisfaisantes.

\section{L'habitat de Champ-Vully à Rances (canton de Vaud, Suisse)}

Cet habitat, qui présente une stratigraphie s'étalant du Néolithique moyen au Bronze moyen, présente dans la phase d'occupation datée du Campaniforme, une architecture et une organisation spatiale proche du site de La Noue. Trois bâtiments, apparemment édifiés sur des cadres de bois et disposés les uns derrière les autres sur deux travées parallèles sont repérables dans la partie fouillée du site (Gallay, Baudais, 1985). Le gabarit des bâtiments est de dimensions moins importantes que pour le site de La Noue $(3,5 \mathrm{~m}$ de largeur et $6 \mathrm{~m}$ de long pour Champ-Vully; 6,5 m de largeur pour $10 \mathrm{~m}$ de longueur pour La Noue). Cet ensemble s'associe à un bâtiment sur poteaux interprété comme un grenier. La différence de dimensions peut s'expliquer par la présence de subdivisions remarquées à l'intérieur des bâtiments de La Noue (locus 2), n'existant apparemment pas à Champ-Vully.

\section{L'habitat de Derrière le Château à Géovreissiat (Ain, France)}

Ce site comporte une série de bâtiments construits sur poteaux, de modules semblables à ceux de La Noue ( $5 \mathrm{~m}$ de largeur sur $11 \mathrm{~m}$ de longueur). Des greniers sur poteaux sont associés aux bâtiments.

\section{L'habitat de Zurich Mozartstrasse (canton de Zurich, Suisse)}

Ce site, daté du Bronze ancien présente un type d'architecture semblable à celui de La Noue et de Champ-Vully. Une série de bâtiments accolés les uns aux autres et disposés sur trois travées parallèles ont été construits sur des cadres de bois mortaisés (Ruoff, 1996). Ces bases sont posées à même la craie lacustre. Les dimensions des bâtiments varient de $4 \mathrm{~m}$ de largeur pour $7 \mathrm{~m}$ de longueur pour le plus petit, à $5 \mathrm{~m}$ sur 10 pour le plus grand. La fouille de l'habitat de Zurich, implanté en milieu humide, a permis la découverte d'éléments architecturaux permettant de reconstituer partiellement l'architecture des bâtiments (bâtiments à toitures à deux pans). Un bâtiment d'orientation différente, interprété comme un grenier, se trouve en limite de l'habitat. 
Tabl. XXVII - Caractéristiques des habitats structurés campaniformes et du Bronze ancien dans les domaines jurassien et alpin.

\begin{tabular}{|c|c|c|c|c|c|}
\hline Site & Architecture & Structures associées & Dimensions des bâtiments & Datation & Contexte géologique \\
\hline $\begin{array}{l}\text { Saint-Marcel } \\
\text { La Noue }\end{array}$ & cadres de bois & $\begin{array}{l}\text { poteaux } \\
\text { fosses dépotoir } \\
\text { vases enterrés }\end{array}$ & $11 m-7 m$ & Campaniforme & $\begin{array}{l}\text { sol alluvionnaire } \\
\text { proximité d'une berge } \\
\text { milieu humide? }\end{array}$ \\
\hline $\begin{array}{l}\text { Rances } \\
\text { Champ-Vully } \\
\text { Niveau 4b }\end{array}$ & cadres de bois & $\begin{array}{l}\text { poteaux } \\
\text { fosses }\end{array}$ & $6,5 m-3,5 m$ & Campaniforme & $\begin{array}{l}\text { replat morainique } \\
\text { galets, graviers } \\
\text { milieu sec }\end{array}$ \\
\hline $\begin{array}{l}\text { Rances } \\
\text { Champ-Vully } \\
\text { Niveau } 4 \mathrm{a}\end{array}$ & cadres de bois & $\begin{array}{l}\text { poteaux } \\
\text { grenier }\end{array}$ & $6,5 m-3,5 m$ & $\begin{array}{l}\text { Campaniforme } \\
\text { Bronze ancien }\end{array}$ & $\begin{array}{l}\text { replat morainique } \\
\text { galets, graviers } \\
\text { milieu sec }\end{array}$ \\
\hline $\begin{array}{l}\text { Zurich } \\
\text { Mozartstrasse }\end{array}$ & cadres de bois & $\begin{array}{l}\text { grenier } \\
\text { sur cadre de bois }\end{array}$ & $\begin{array}{l}4,5 m-6 m \\
5 m-10 m\end{array}$ & Bronze ancien & $\begin{array}{l}\text { berge de lac } \\
\text { craie lacustre } \\
\text { milieu humide }\end{array}$ \\
\hline Géovreissiat & poteaux & $\begin{array}{l}\text { grenier } \\
?\end{array}$ & $5 m-11 m$ & $\begin{array}{l}\text { Campaniforme } \\
\text { Bronze ancien }\end{array}$ & vallon sec perché \\
\hline
\end{tabular}

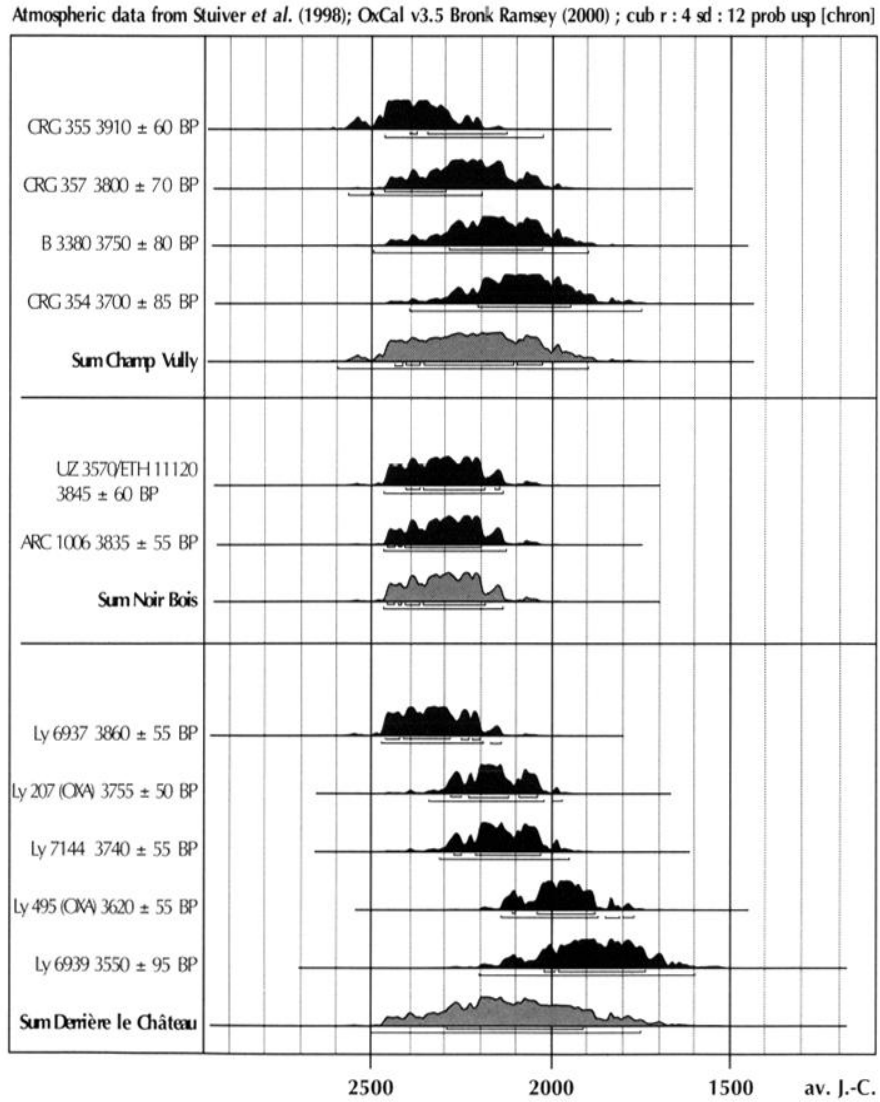

Fig. 70 - Datation du groupe bourguignon-jurassien. Sum: somme des probabilités.

Le tableau XXVII montre une forte utilisation de l'architecture sur cadre de bois dans les domaines jurassien et alpin du Campaniforme au Bronze ancien. Au-delà d'une adaptation au milieu fréquemment humide dans lequel sont implantés ces habitats, il est possible d'interpréter ce phénomène en terme culturel. Le site de Champ-Vully, édifié en milieu sec et qui dispose malgré tout d'une architecture sur cadre de bois évoluant du Campaniforme au Bronze ancien, en est l'illustration. Il semble que ce type d'architecture soit abandonné au profit de la technique plus classique des poteaux dès le Bronze moyen sur le site de Champ-Vully.

F. D.

\section{Datations absolues}

Les datations réalisées sur les trois sites de référence montrent que ces habitats sont tardifs dans la séquence du Campaniforme (2400/2300-2200/1900 av. J.-C.), même Champ-Vully qui a pourtant livré un assemblage céramique différent renvoyant au standard du Campaniforme européen (fig. 70). Ces datations s'insèrent dans le schéma proposé à l'échelle européenne: les dates les plus anciennes proviennent du sud-ouest de l'Europe; l'est de la France et la Suisse sont atteints tardivement par le Campaniforme. (Bailly, Salanova, 1999).

Quant au site de La Noue, rien n'autorise à penser que sa place chronologique soit différente de celle de Noir Bois et de Derrière le Château; il est même très probable que ces trois sites soient contemporains.

L. S.

\section{CARACTÉRISTIQUES DU GROUPE BOURGUIGNON-JURASSIEN}

Le groupe bourguignon-jurassien est désormais défini sur la base solide d'habitats étudiés récemment. 
Champ-Vully à Rances montre des différences avec les autres ensembles, tant au niveau de la culture matérielle qu'au niveau économique. Nous imputons ces différences à un facteur chronologique: l'habitat de Rances nous semble plus ancien que les trois autres sites. Noir Bois à Alle, Derrière le Château à Géovreissiat et La Noue à SaintMarcel partagent au contraire un certain nombre de points communs qui sont au cœur de la définition du groupe bourguignon-jurassien.

Les habitats se présentent sous la forme de petits villages dont aucun n'a été fouillé intégralement. Les unités d'habitations, de forme quadrangulaire, sont toutes de petit module. Elles ne se distinguent en rien des modèles connus aux époques antérieures et postérieures dans la région. Le spectre matériel est cependant différent. Cette rupture dans la culture matérielle plaide en défaveur d'une évolution continue ancrée dans les traditions locales. Nous refusons cependant de n'interpréter cette rupture qu'en termes ethniques: la gestion des matières premières montre bien que les populations établies dans ces habitats connaissent parfaitement leur environnement.

En ce qui concerne les aspects matériels, l'industrie lithique est dominée par les grattoirs et les pièces esquillées. Le spectre céramique est composé de gobelets, et dans une moindre mesure de formes basses, ornés par impression de peigne à l'aide d'ın répertoire ornemental restreint (lignes horizontales en thème unique, échelles, bandes hachurées et chevrons). La céramique commune est caractérisée par les types les plus fréquents à l'échelle de l'Europe occidentale: jarres à cordons et formes basses (bol, écuelle, jatte). Dans l'industrie lithique comme dans la céramique, les attributs du groupe bourguignon-jurassien sont relativement ubiquistes dans le Campaniforme européen. Seule leur association récurrente et monotone est significative.

Les variations enregistrées dans la culture matérielle de ces trois sites sont imputables à des influences externes perceptibles dans le cortège céramique et dans les analyses de matériaux. Au nord de la région, les influences centreeuropéennes sont plus virulentes, comme l'illustre le corpus de Noir Bois à Alle. Au sud, l'assemblage de Derrière le Château à Géovreissiat montre davantage d'affinité avec les groupes campaniformes méridionaux. La Noue à SaintMarcel, situé géographiquement entre les deux précédents sites, est peu atteint par le courant méridional. En revanche, les influences d'Europe centrale y sont sensibles.

Nous avions déjà souligné, dans le cadre d'une autre étude, que certains assemblages provençaux présentent les mêmes caractéristiques (Salanova, 1997). La série découverte à La Balance en Avignon en est la plus claire illustration: coupe d'obédience méridionale ornée de décor monotone de lignes et de chevrons. Cet exemple montre le rôle dynamique, récepteur mais aussi émetteur, du groupe bourguignon-jurassien au carrefour de deux courants majeurs dans le Campaniforme européen: l'Europe centrale et la Méditerranée.

L. S.

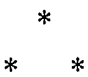

Le bon état de conservation du site de La Noue permet un renouvellement considérable des connaissances sur le Campaniforme bourguignon et, bien au-delà, sur les habitats campaniformes en général.

Les observations de terrain et les études de mobilier s'accordent sur la relative homogénéité du site. Contrairement au mobilier céramique pour lequel des remontages ont été réalisés d'un locus à l'autre, le matériel lithique taillé est exempt de raccord d'une maisonnée à l'autre. Le corpus de silex est néanmoins très homogène. Il est impossible de préciser la durée d'occupation du site, mais aucun élément ne plaide en faveur d'une durée d'occupation longue.

Nos conclusions penchent en faveur d'un habitat à caractère durable, mais son occupation a pu être discontinue, selon un rythme saisonnier. Au moins deux locus (2 et 6) comportent des assemblages lithiques témoignant d'activités spécialisées, peut-être en rapport avec la présence de cet habitat. L'analyse des argiles met en évidence un groupe de vases exogènes, reliant le site de La Noue aux habitats situés en rive droite de la Saône et implantés en hauteur. Une complémentarité économique entre les deux zones expliquerait la situation particulière de La Noue.

En effet, bien que les analyses paléoenvironnentales fassent défaut, plusieurs indices plaident en faveur d'inondations sur le site. Les effets de parois, nettement lisibles sur le terrain, vont dans ce sens, de même que le remplissagc discontinu de certaines fosses dépotoirs. Les silex, très rarement patinés, présentent un état de conservation exceptionnel qui induit un recouvrement assez rapide du site. L'absence de flottage et de courant est de mise pour l'ensemble des vestiges. Il est probable qu'une crue rapide et ultime ait maintenu le mobilier dans un cadre, ce qui sous-tend une architecture en bois, fondée sur sablières basses. La submersion et l'abandon du site se sont sans doute déroulés dans un délai bref, expliquant la bonne conservation des structures et du mobilier. 
L'architecture et l'organisation spatiale de La Noue se retrouvent dans l'aire jurassienne à la même période. La série céramique s'insère totalement dans le groupe campaniforme bourguignon-jurassien. Sur la base des artefacts siliceux, l'hypothèse qui consisterait à qualifier La Noue de site de contrôle, de diffusion et d'échange avec les groupes campaniformes voisins, peut être écartée, contrairement à ce qui a été proposé pour le gisement de Champ-Vully à Rances (Bailly, 2003). Concernant la céramique, la plupart des récipients trouvent certes des comparaisons en Bourgogne orientale. Toutefois, un ensemble de vases, bien que fabriqué régionalement, est caractéristique du domaine oriental et septentrional de l'Europe campaniforme. Ces vases sont concentrés dans les deux locus qui ont également livré des assemblages lithiques liés à une activité spécialisée. Nous serions tentés d'y voir un lien et d'envisager l'installation d'artisans spécialisés d'origine extra-régionale sur le site de La Noue.

La comparaison des données dans un cadre régional large permet de poser les bases de la définition d'un nouveau groupe du Campaniforme régional. Ce groupe «bourguignon-jurassien " est mieux connu en contexte domestique. Il présente un spectre matériel homogène et relativement monotone. Sa position stratégique le long de l'axe Saône-Rhône doit expliquer son rôle de vecteur d'influences méditerranéennes et centre-européennes. Ses contours, conditionnés par les travaux de terrain, restent cependant à délimiter plus précisément. Sa frontière méridionale notamment semble se fondre dans le groupe provençal. De même, les mécanismes liés à sa formation restent peu clairs. Certains éléments s'intègrent parfaitement dans la tradition régionale, mais le domaine de la culture matérielle marque une franche rupture. Si une partie, toujours minoritaire, des vases découverts dans les habitats du groupe bourguignon-jurassien s'avère exogène, l'arrivée d'une population étrangère ne peut dans aucun des cas connus expliquer à elle seule leur implantation. Dans l'est de la France, manquent les jalons entre l'abandon des rives des lacs et l'édification de ces habitats terrestres pour cerner toutes les étapes de la fin du Néolithique. Les études environnementales les plus récentes montrent toutefois que ces deux épisodes sont consécutifs à un phénomène climatique majeur qui a très probablement eu des répercussions sur l'occupation du sol et sur les réseaux d'échange (Delhon et al., à paraître). Sans être totalement déterminant, ce phénomène, dont les conséquences ont dû varier selon les milieux, pourrait expliquer les différences notoires repérées dans la nature des occupations campaniformes d'un bout à l'autre de l'Europe. Si dans le groupe bourguignon-jurassien le Campaniforme marque effectivement un certain renouveau des implantations domestiques, ce qui a été interprété par A. Gallay en termes migrationnistes, rien de tel n'est sensible dans les vestiges campaniformes d'autres zones, comme la péninsule Ibérique. Ce polymorphisme interdit toute tentative d'interprétation globale du Campaniforme qui doit sans cesse être décomposé pour en cerner toutes les subtilités.

Collectif 


\section{BIBLIOGRAPHIE}

\section{AFFOLTER J.}

1997 : " Approche typologique et technologique de l'industrie lithique ", in OTHENIN-Grin B. (DIR.), Le Campaniforme d'Alle, Noir Bois (Jura, Suisse), Porrentruy, Office du Patrimoine historique et Société jurassienne d'émulation (Cahiers d'archéologie jurassienne, 7), p. 116-121.

2003 : "L'industrie lithique taillée: la provenance des matières siliceuses ", in BEsst M. (DIR.) : Le Campaniforme de Champ-Vully a Rances (Vaud, Suisse), rapport, Université de Genève, p. 18-19.

\section{argant J., Chastel J., Ferrier C.}

1996 : "La moyenne vallée de la Saône il y a 6000 ans, archéologie et paléoenvironnement ", Revue archéologique de l'Est, 47, p. $35-45$.

\section{ARmand-Calliat L.}

1962 : "Poignard en cuivre et objets métalliques de l'âge du Bronze, trouvés en Chalonnais ", Revue archéologique de l'Est, XIII, p. 289-298.

\section{ARNOLD D. E.}

1985 : Ceramic Theory and Cultural Process, Cambridge University Press, Cambridge, $268 \mathrm{p}$.

\section{Astruc L.}

1997 : « Analyse tracéologique de quelques artefacts en silex ", in OtHENIN-Grinis B. (DIR.) : Le Campaniforme d'Alle, Noir Bois (Jura, Suisse), Porrentruy, Office du Patrimoine historique et Société jurassienne d'émulation (Cahiers d'archéologie jurassienne, 7), p. 121-127.

\section{BAILLY M.}

1997-1998: "L'industrie lithique taillée de Derrière-le-Château: intérêts et paradoxe ", Archéologia, hors série 9, p. 50-54.

2002a: "Du Néolithique final à l'âge du Bronze ancien en Bassin rhodanien, une première approche du statut des productions lithiques ", in BaIlLY M., Furestitr R., Perrin T. (DIR.): Les Industries lithiques taillees holocènes du Bassin rhodanien: problèmes et actualités, Actes de la table ronde de Lyon, 8-9 déc. 2000, Montagnac, éd. Monique Mergoil, (coll. Préhistoires), p. 51-68.

2002b: La Flèche et l'éclat: production et consommation des outillages lithiques taillés de la fin de Néolithique au dérnt de l'âge du Bronze entre Saône et Rhône (2600-2000 au. J.-C.), thèse de doctorat en Archéologie, univ. de Besançon, 2 vol., 507 p. et 1 vol. ill.

2003 : "L'industrie lithique taillée: Analyse et typologie ", in BESSE M., Le Campaniforme de Champ-Vully à Rances (Vaud, Suisse), rapport, Université de Genève, p. 19-25.

\section{BaILly M., Besse M.}

2004 : « Le complexe campaniforme rhodanorhénan: suite et fin? " in DakTevelle $\mathrm{H}$. (F.D.): : Actes des $5^{\circ}$ rencontres méridionales de Préhistoire récente Auvergne et Midi, ClermontFerrand, nov. 2002, Cressensac, Préhistoire du Sud-Ouest, Suppl. 9.

\section{Bailiy M., Salanova L.}

1999 : " Les dates radiocarbone du Campaniforme en Europe occidentale: analyse critique des principales séries de dates ", in Évin J., OBeri.IN C., ${ }^{44} \mathrm{C}$ el Archéologie, Actes du $3^{2}$ congrès innervationl, Lyon, 6-10 avr. 1998, Mémoires de la Société préhistoriques française, XXVI, et suppl. à la Revue d'Archéométrie, p. 219-224.

\section{BAISE L.}

1996 : Approches architecturales des sépultures collectives mégalithiques de Bourgogne: l'exemple de la Cöte-d'Ur, mém. de DEA., univ. de Bourgogne, Dijon, 2 vol., 51 p., 40 pl.

\section{Barge-Mahieu $\mathbf{H}$}

1989 : « L'habitat perché et les cabanes campaniformes des Calades (Orgon, Bouchesdu-Rhône) ", in Enceintes, habitats ceinturés, sites perchés du Néolithique au Bronze ancien dans le sud de la France et les régions voisines, Mémoires de la Société languedocienne de préhistoire, 2, p. 231-237.

\section{BESSE M.}

1996a: Le Campaniforme en France: analyse de la céramique d'accompagnement, Oxford, BAR, International Series, 635,56 p., 115 fig., 26 pl. h. t.

1996b: «Types et origines potentielles de la céramique d'accompagnement du Campaniforme en France ", in Mordant C., GaIfFE O. (ÉD.): Cultures et sociétés du Bronze ancien en Europe, Actes du $117^{\circ}$ congrès national des sociétés savantes, Clermont-Ferrand, 1992, Paris, éd. du Comité des travaux historiques et scientifiques, p. 165-180.

2003a: L'Europe du $3^{*}$ millénaire avant notre ère: les céramiques communes au Campani- forme, Lausanne, Cahiers d'archẹologie romande, 94, 223 p., $1 \mathrm{CD}$

2003b: "Les céramiques communes des Campaniformes ", Gallia Préhistoire, 45, p. 205-258.

BESSE M. (DIR.)

2003 : Le Campaniforme de Champ-Vully à Rances (Vaud, Suisse), rapport, Lniversité de Genève.

BILL J.

1973 : Die Glockenbecherkultur und die frühe Bronzezeit in französischen Rhonebecken und ihre Beziehungen zur Südwestschweiz, Basel, Société suisse de préhistoire et d'archéologie, 111 p., 15 cartes, $63 \mathrm{pl}$.

Billard C., Bourhis R., Desfosses Y., Évin J., Huault M.-F, Lefebve D., PauletLOCARD M.-A.

1991: " Nouveaux sites campaniformes de la basse vallée de la Seine. Présentation et localisation des sites, I. L'habitat des Florentins à Val-de-Rueil (Eure) ", Callia Préhistoire, 33, p. 140-171.

\section{BLanc A.}

1958: « Découverte d’une sépulture campaniforme sur la commune de Sovons (Ardèche) ", Cahiers rhodaniens, 5, p. 52-54.

\section{BonNamour L.}

1969 : L'Âge du Bronze au musée de Chalon-surSaône, Mâcon, 31 p., 8 pl.

1975 : "Chroniques archéologiques", Mémoires de la Société historique et archéologique de Chalon, XIV, p. 27-28.

Bonnamour L., Marinval P., Marguerie D., Lambert G.-N., LaVier C., Poulain T.

1990 : "Les habitants du Bronze final III dans la vallée de la Saône au sud de Chalon-surSaône et le Gué-des-Piles ", in Un monde villageois. Habitat et milieu naturel en Europe de 2000 à 500 av. J.-C., Lons-le-Saunier, p. $97-111$.

\section{Bonnamour L., Moyne G.}

1963 : " Un nouveau site chalcolithique dans le Val de Saône: Le Breuil à Saint-Marcel (S.et-L.) ", Revue archéologique de l'Est, XIV, p. 141-149.

BonTEMPS C.

1997 : Ouroux-sur-Saône "Le Petit Bois", 
Document final de synthèse d'évaluation archéologique, Dijon, SRA Bourgogne, 49 p. non foliotées, 50 fig. et 2 p. de tabl.

\section{Bonvalot J., Fleury R., Gri ard J.-P.}

1984 : "Chalon-sur-Saône ", carte au 1/25000, Orléans, éd. du Bureau de recherches géologiques et minières, p. 63.

1984 : « Notice explicative ", Carte géologique de la France $(1 / 50000)$, feuille Chalonsur-Saône (579), Orléans, Bureau de recherches géologiques et minières, $63 \mathrm{p}$., Carte géologique par Bonvalot J., Gelard J.-P., Menot J.-C., Fleury R. (1982).

\section{BOUILLEROT R.}

1932 : " La période néolithique à l'Abergement-les-Auxonne ", Mémoires de la Commission des Antiquités de la Côte-d'Or, XIX, 1927-1932, p. 58-68.

\section{BOURa F.}

1993 : " Découverte d'un site d'habitat campaniforme à Vandières (Meurthe-etMoselle): premiers résultats ", in $L e$ Néolithique du nord-est de la France et des régions limitrophes, Actes du XIIr colloque interrégional sur le Néolithique, Metz, 10-12 oct. 1986, Paris, éd. de la Maison des sciences de l'Homme (Documents d'archéologie française, 41), p. 165-172.

\section{Cabrol. A.}

1939 : "Bronzes des dragages de la Saône ", Bulletin de la Société préhistorique franşaise, X, p. 2.

\section{Chastel J., Jallet F., Nourissat S.}

2003 : " Mésolithique et Néolithique (10000-2200 avant J.-C.) 》. in Poux M. (DIR.), Lyon avant Lugdunum, catalogue d'exposition, Lyon, éd. Infolio, p. $36-45$

\section{Clair A.}

1982 : " Notice explicative ", Carte géologique de la France $(1 / 50000)$, feuille Dijon $(500)$, Orléans, Bureau de recherches géologiques et minières, 52 p., Carte géologique par Clatr A., Vermi P., Puisséciur J.-J.

\section{Colomer A., Coularou J., Gutherz X.}

1990 : Boussargues (Argelliers, Héraull). Un habitat ceinturé chalcolithique: les fouilles du secteur ouest, Paris, éd. de la Maison des sciences de l'Homme (Documents d'archéologie française, 24), 220 p., 132 fig.

\section{Combier J.}

1959 : "Informations archéologiques, Circonscription de Lyon ", Gallia Préhistoire, II, fasc. 1, p. 23.

1962 : " Informations archéologiques, Circonscription de Lyon ", Gallia Préhistoire, V, fasc. 1, p. 300-302.

\section{Convertini F.}

1996 : Production et signification de la céramique campaniforme à la fin du IIT millénaire av. J.-C. dans le Sud et le Centre-Ouest de la France et en Suisse occidentale, Oxford, BAR, International Series, 656, 362 p., 111 fig., 71 tabl., 2 pl. h. t.

1997a: "Origine des ressources argileuses ", in OTHENIN-GIRARD B. (DIR.), Le Campaniforme d'Alle, Noir Bois (Jura, Suisse), Porrentruy, Office du Patrimoine historique et Société jurassienne d'émulation (Cahiers d'archéologie jurassienne, 7), p. $70-84$.

1997b: " La céramique campaniforme sous le microscope " in "Rhône-Alpes A 404: L'énigmatique civilisation campaniforme ", Archéologia, hors-série 9, p. 56.

2001 : "Production de la céramique campaniforme dans l'axe Rhin-Rhône ", in Nicol.IS F. (ÉD.) : Bell Beakers Today, Pottery, People, Culture, Symbols in Prehistoric Europe, Proceedings of the International Colloquium, Riva del Garda, Trento, 11-16 May' 1998, Provincia autonome di Trento (Italia), Servicio beni culturali, Ufficio beni archeologici, p. 547-560.

2003 : " Les matériaux: analyse en lames mines de vases ", in BFSSF. M. (DIR.), Le Campaniforme de Champ-Vully à Rances (Vaud, Suisse). rapport, Université de Genève, p. 6-13, 3 fig.

Convertini F., Othenin-Girard B.

1997 : "Analyse techno-culturelle ", in OTHFNIN-GIRARD B. (DIR.), Le Campaniforme d'Alle, Noir Bois (Jura, Suisse), Porrentruy, Office du Patrimoine historique et Société jurassienne d'émulation (Cahiers d'archéologie jurassienne, 7), p. 85-91.

Courel L., Arene J., Feys R., Gand G., Menot J.-C., Cornet J., Guillaumet J.-P.

1989 : " Notice explicative, Carte géologique de la France $(1 / 50000)$, feuille Le Creusot (552) ", Orléans, Bureau de recherches géologiques et minières, 54 p., Carte géologique par Fournigurt J., Courfi. L., Gand G., Menot J.-C., Fhis R., ARFNF. J., GUHLLAUMET J.P. (1988).

\section{CourTors L.}

1971 : Description physico-chimique de la céramique ancienne: la céramique de Chypre au Bronze récent, thèse de $3^{\mathrm{e}}$ cycle, univ. de ClermontFerrand, $182 \mathrm{p}$.

Del.hon C., Salanova L., Bfrger J.-F., Thrébault S.

À paraître: «Évolution du milieu et du climat au Campaniforme: nouvelles hypothèses ", in Actes du 129 congrès des Sociétés historiques et scientifiques, Besancon, avr. 2004, thème 5 " Emprises, déprises et rythmes agricoles à l'âge du Bronze ».

\section{DETREY J.}

1997 : « Approche typologique et technologique de l'industrie lithique ", in OTHenin-Genin B. (DIR.), Le Campaniforme d'Alle, Noir Bois, Porrentruy, Office du patrimoine historique et Société jurassienne d'émulation (Cahier d'archéologie jurassienne, 7), p. 95-115.

2002 : " Mise en évidence d'une composante régionale dans l'industrie lithique campaniforme d'Ajoie (Jura Suisse) ", in Bail..y M., Furestier R., Perrin T. (DIR.), Les Industries lithiques taillées holocènes $d u$ bassin rhodanien, problèmes et actualités, Actes de la table ronde de Lyon, 8-9 déc. 2000, Montagnac, éd. Monique Mergoil, (coll. Préhistoires), p. 181-204.

2003 : "Un exemple de gestion des matières premières au Campaniforme: l'industrie lithique du site d'Alle, Noir Bois (Jura Suisse)". Bulletin de la Société préhistorique française, 100, 2, p. 393-405.

Dorion J., Buvot P., Gauthey J.

1987 : "Le coffre mégalithique du Poiset", Revue archéologique de l'Est, XXXVIII, p. $443-456$.

\section{DUCREUX F, AmRane Y.}

1996 : La Déviation de la RN 73 à Saint-Marcel. Sauvetage des sites de La Noue et Sur le Bief, Document final de synthèse, Dijon, SRA Bourgogne.

DUCREUX F., LABEAUNE R.

1999 : Liaison Arc-sur-Tille - Dijon, Rapport de diagnostic, Dijon, SRA Bourgogne.

\section{DufaY-Galan A.}

1996 : "Le Néolithique moyen de la grotte de Molle-Pierre, Pavilly-Mandelot (Côted'Or): un ensemble NMB ", in $L a$ Bourgogne entre les bassins rhénan, rhodanien et parisien: carrefour ou frontière? $18^{\circ}$ colloque interrégional sur le Néolithique, Dijon, 25-27 oct. 1991, résumés des communications, Dijon, SRA Bourgogne, p. 23. 
DuRIAUd J.

1997a: " La station campaniforme de La Croix Léonard (Tournus) ", in 30 ans d'archéologie en Saône-et-Loire, catalogue d'exposition, Dijon, Comité départemental de la recherche archéologique, p. $80-81$.

1997b: "Les stations néolithiques de BoisDerrière et Beauvois ", in 30 ans d'archéologie en Saône-et-Loire, catalogue d'exposition, Dijon, Comité départemental de la recherche archéologique, p. 78-79.

\section{ÉCHALLIER J.-C.}

1984 : Éléments de technologie céramique et d'analyse des terres cuites archéologiques. Méthodes et techniques, Lambesc, Documents d'archéologie méridionale, 3 , $39 \mathrm{p}$.

\section{FiLLION J.-P.}

2002 : "Campaniforme et industrie lithique. Les sites de "En Chatanay" et "En Lachat" dans le bassin bellegardien (Ain) ", in Bail.ty M., Furfestier R., Perrin T. (Dir.), Les Industries lithiques taillées holocènes $d u$ bassin rhodanien, problemes et actualités, Actes de la table ronde de Lyon, 8-9 déc. 2000, Montagnac, éd. Monique Mergoil (coll. Préhistoires), p. 155-165.

Fleury R., Gelard J.P. et collab.

1983 : Notice explicative, Carte géologique de la France $(1 / 50000)$, feuille Chagny (553), Orléans, Bureau de recherches géologiques et minières, 81 p., Carte géologique par Fl.eury R., GeI.ARD J.-P., Menot J.-C., Pascal A., Bonvalot J., Ciozier L., Thévenot J.-P., Delance J.-H., Amiot M., Beauduc P. (1982).

\section{FOUÉRÉ P.}

1994 : Les Industries en silex entre Néolithique moyen et Campaniforme dans le Nord $d u$ bassin aquitain: approche méthodologique, implications culturelles de l'économie des matière premières et du débitage, thèse de doctorat, univ. de Bordeaux-I, $\mathrm{n}^{\circ} 1188$, 547 p., 163 fig., 139 pl. h. t.

\section{FoUt́ré P., ROGER J.-M.}

2002 : « Le matériel lithique du site campaniforme de Maupas (Calvisson, Gard) ", in Bailly M., Furestier R., Perrin T. (Dir.), Les Industries lithiques taillées holocènes du bassin rhodanien, problemes et actualités, Actes de la table ronde de Lyon, 8-9 déc. 2000, Montagnac, éd. Monique Mergoil (coll. Préhistoires), p. 225-237.

\section{FURESTIER R.}

2002 : « Y-a-t-il une production spécifique de support d'outil chez les Campaniformes du Sud-Est de la France? Premières caractérisations pour la Provence ", in BaII.IY M., FirestIER R., PERRIN T. (DIR.), Les Industries lithiques taillées holocènes du bassin rhodanien, problèmes et actualités, Actes de la table ronde de Lyon, 8-9 déc. 2000, Montagnac, éd. Monique Mergoil (coll. Préhistoires), p. 167-179.

\section{Gallay A.}

1986 : " Autonomie du campaniforme rhodano-rhénan, la question de la céramique domestique ", in Drmoul.E J.-P., Gull.Aine J. (DIR.), Le Néolithique de la France. Hommage à G. Bailloud, Paris, éd. Picard, p. 431-446.

\section{Gallay A., Baudais D.}

1985 : "Rances, Champ-Vully Est (Vaud, Suisse) ", in Première céramique, premier métal: du Néolithique à l'âge du Bronze dans le domaine circum-alpin, catalogue d'exposition du musée de Lons-le-Saunier (oct. 1985-mars 1986), Lons-le-Saunier, Musée municipal d'Archéologie, p. 99-108.

\section{Gallay A., Baudais D., Boisset C.}

1980 : " Rances, distr. D'orbes, VD. CampVully ", Annuaire de la Société suisse de préhistoire et d'archéologie, 63, 1980, p. 233-236.

\section{GaRDINI M.}

2000 : "Permanence et déplacements du lit mineur de la Saône: l'exemple de la région châlonnaise ", in BONNAMOUR L. (DIR.), Archéologie des fleuves et des rivières, Paris, éd. Errance, p. 11-14.

Gaudron G.

1956 : "Vase inédit de l'âge du Bronze ", Bulletin de la Société préhistorique fransaise, 53, p. $464-465$.

Gosselain O. P.

2002 : Poteries du Cameroun méridional. Styles techniques et rapports à l'identité, Paris, CNRS Éditions (coll. CRA Monographie), 26, 254 p., 162 fig.

\section{Gros A.-C.}

1966 : « Découverte d'une importante station préhistorique dans la plaine de Crissey (Saône-et-Loire) ", Mémoires de la Sociêté historique et archéologique de Chalon-sur. Saône, XXXVIII, p. 15.

Gros A.-C., Gros O.

1968 : " La céramique caliciforme dans la vallée de la Saône ", Terre Vive, 4, p. 9-15.
Gros A.-C., ThÉvenot J.-P.

1957 : “Une station néolithique inédite à Ouroux-sur-Saône ". Mémoires de la Société historique et archéologique de Chalon-surSaône, XXXIV, fasc. 2, p. 102-107.

\section{GuYot E.}

1940 : "Dolmen et site de Ternant ", Bulletin archéologique du Comité des travaux historiques (1934-1935), p. 443-456.

\section{HÉNON P., VÉrot-BourRÉLY A.}

1998 : “ Habitats néolithiques, protohistoriques et occupations historiques du site de "Derrière-le-Château", à Géovreissiat et Montréal-La-Cluse ", Ain, France, in Frère-SAUTOT M.-C. (DIR.) : Paléométallurgie des cuivres, Actes du colloque de Bourg-en-Bresse et Beaune, 17-18 oct. 1997, p. $207-249$.

\section{JEANNET A.}

1970 : "Quelques objets en pierre polie du Mâconnais ", Bulletin de la Société préhistorique fransaise, 67 , p. 522-529.

\section{JOLIBERT B.}

1988 : Le gisement campaniforme de Muret, Toulouse, EHESS (Archives d'écologie préhistoriques, 8), $134 \mathrm{p}$.

\section{JoLY J. ABBÉ}

1968 : " Compte-rendu de la circonscription de Bourgogne ", Gallia Préhistoire, XI, fasc. 2, p. 378-379.

1970 : «Quelques précisions sur les tumulus et abris situés à l'est de Fleurey-sur-Ouche ", Revue archéologique de l'Ouest, XXI, fasc. 1-2, p. 121-128.

Joly J. ABbé, PuissÉgur J.J.

1965 : « Les tombes mégalithiques de la Côted'Or ", Congrès préhistorique de France, XVT session Monaco, 1959, Paris, Société préhistorique française, p. 688-702.

Kосн B., Boissonnas J., Feys R., Alabouvette B., Vincent P.-L., Cornet J.

1976 : Notice explicative, Carte géologique de la France $(1 / 50000)$, feuille Montceaules-Mines (578), Orléans, Bureau de recherches géologiques et minières, $42 \mathrm{p}$., Carte géologique par BoIssonnas J., Fers R., Aiabouvette B., Vincent P.-L., CORNET J. (1976).

Labeaune R., Ducreux F., Staniaszek L.

2003 : Longvic-sur-Onges, "Les Quétignières ", Rapport final d'opération, 2 vol., Dijon, SRA Bourgogne. 
LETTERLÉ F., VerRon G.

1986 : "Un site d'habitat campaniforme à Diguleville (Manche) ", Revue archéologique de l'Ouest, suppl. 1, p. 237-252.

\section{LONGIN R.}

1971 : "New méthode of collagen extraction for radiocarbon dating ", Nature, 230, p. 241-242.

NICOLARDOT J.-P.

1968 : La Protohistoire de la montagne beaunoise, thèse dactylographiée, univ. de Bourgogne, Dijon.

\section{OTHENIN-GIRARD (DIR.)}

1997 : Le Campaniforme d'Alle, Noir Bois (Jura, Suisse), Porrentruy, Office du Patrimoine historique et Société jurassienne d'émulation (Cahiers d'archéologie jurassienne, 7), $208 \mathrm{p}$.

\section{Pétrequin P. (DIR.)}

1997 : Chalain station 3 (3200-2900 av. J.-(.) -III- Les sites littoraux nóolithiques de Clairvaux-Les-lacs et de Chalain (Jura), Paris, éd. de la Maison des sciences de l'Homme, (coll. Archéologie et culture matérielle), $765 \mathrm{p}$.

\section{Philibert D.}

1967 : Une station tardenoisienne dans le Beaujolais a Boitrait, commune de SaintGeorges-de-Reneins (Rhône), Document des laboratoires de géologie de la faculté des sciences de Lyon.

\section{RAMSex C. B.}

2000 : "Comments on "The Use of Bayesian Statistics for ${ }^{14} \mathrm{C}$ dates of chronologically ordered samples: a critical analysis" ", Radiocarbon, 42, 2, p. 199-202.

RÉmond C. avec la collab. de Blanalt J.-C., BIGOT M.

1985 : Notice explicative, Carte géologique de la France $(1 / 50000)$, feuille Beaune (526), Orléans, Bureau de recherches géologiques et minières, 53 p., Carte géologique par Rimond C., Gunot B., BRIOTET H., BRIZARD F., MENot J.-C., Rogues (;., Sant-AMant F. (1984).

\section{Riquet R., Guilaine J., Coffyn A.}

1963 : "Les Campaniformes français ", Giallia Préhistoire, VI, p. 63-128.

\section{ROINÉ N.}

1992 : Déviation de la $R N 73$, diagnosíic archéologique, Dijon, SRA Bourgogne.
RoupIL J.-L.

1992 : « L'habitat du Bois Sacré à Saint-Cômeet-Maruejols (Gard) ", in BARcikMantlic H. (DIR.), le Campaniforme dans le midi de la France: origine et identité culturelle, catalogue d'exposition, monastère de Notre-Dame de Beauregard (Orgon), Marseille, éd. EPA, p. 55-57.

\section{Ruorf U.}

1996 : " Meilen-Schellen et ZurichMozartstrasse, deux sites lacustres au bord du lac de Zurich ", in MORDonNT C., GalfF: O. (ÉD.): Cultures el sociétés $d u$ Bronze ancien en Europe, Actes du $117^{\circ}$ congrès national des Sociétés savantes, Clermont-Ferrand, 1992, Paris, éd. du Comité des travaux historiques et scientifiques, p. 199-210.

\section{SaINT-Jean-Virtus B.}

2000 : Quétigny, les Allées Cavalières, Rapport de diagnostic, Dijon, SRA Bourgogne.

\section{SaINTOT S.}

1999 : « Les armatures de flèches en silex de Chalain et de Clairvaux ", in PÉtrroutn P. (DIR.), "Parures et flèches du Néolithique final à Chalain et Clairvaux (Jura): une approche culturelle et environnementale ", Gallia Préhistoire, 40, p. 204-241.

\section{SALANOva $\mathbf{L}$.}

1996 : Des Cloches et des coquillages... Fabrication et ornementation des vases campaniformes en France, thèse de doctorat en PréhistoireEthnologie-Anthropologie, univ: Paris-I, 2 vol., $1009 \mathrm{p}$.

1997 : La Céramique décorée du sile campaniforme de Derrière-le-Château (Géoureissiat, Ain), rapport, Lyon, SRA Rhône-Alpes.

2000: La Question du Campaniforme en France et dans les iles anglo-normandes: productions, chronologie et rôles d'un standard céramique, Paris, éd. du Comité des travaux historiques et scientifiques (Documents préhistoriques) et Société préhistorique française, $391 \mathrm{p}$.

2003 : " La céramique décoréc ", in Besse M. (DIR.), Le Campaniforme de ChampVully à Rances (Vaud, Suisse), rapport, Université de Genève, p. 13-15.

\section{SÉara F., Ganard V. (DIR.)}

1996: Les Gisements de Choisey "Aux (hampins" et de Ruffey-sur-Seille "À Daupharde " (Jura), Etudes des occupations mésolithiques, néolithiques et protohistoriques de denx sites de plaine alluviale, Document final de synthèse de fouille préventive, Besançon, SRA Franche-Comté, $569 \mathrm{p}$

Sigaud F.

1991 : "Un couteau ne sert pas à couper mais en coupant. Structure, fonctionnement et fonction dans l'analyse des objets ", in 25 ans d'études technologiques en Préhistoire, $X I$ rencontres internationales d'archéologie et d'histoire d'Antibes, 18-20 oct. 1990, Juanles-Pins, éd. APDCA, p. 21-34.

STRAHM C. (ÉD.)

1995: Das Glockenbecher-Phänomen. Ein Seminar. Institut für Ur- und Frühgeschichte der Universität Freiburg im Brisgau (Freiburger archäologiche Studien, 2), $409 \mathrm{p}$.

StUIVER M., Reimer P. J., Bard E. ET al. 1998 : «INTCAL 98 Radiocarbon Age Calibration, 2400-0 cal BP ", Radiocarbon, 40,3 , p. $1041-1083$.

THÉVENOT J.-P.

1961 : "Le tumulus 1 de Vertempierre à Chagny (Saône-et-Loire) ", Revue archéologique de lEst, XII, fasc. II, p. 164-169.

1973 : Le Village préhistorique d'Ouroux-sur-saône, Travaux du Centre de recherches de Solutré, 1, 174 p., 91 fig.

1978 : " Information archéologiques ", Gallia Préhistoire, 21, fasc. 2, p. 580.

1990 : «Le Camp de Chassey et le Néolithique de Bourgogne orientale ", in Die Erstern Bayern, Zurich, I andesmuseum, 2 vol., p. 197-204.

Thévenot J.-P., Strahm C., Pétrequin P., BocQuet A., Schifferdecker. F., Beeching A., Gallay A., Bill J.

1976 : "La civilisation Saône-Rhône ", Revue archéologique de l'Est, 27, p. 331-420.

Treinen F.

1970 : "Les poteries campaniformes en France ", Gallia Préhistoire, XIII, fasc. 1. p. 53-106; fasc. 2, p. 263-332.

Trémeau B.

1984 : "Le site naturel de Chalon-sur-Saône ", Mémoires de la Société d'histoire et d'archéologie de Chalon-sur-Saône, 53.

VIRLOGEUX Y. (DIR.)

2002 : Chevigny-Saint-Sauveur (2l) ZAC "Excellence 2000 ". Fouille de sausetage archéologique. Établissements ruraux gaulois et gallo-romains, Rapport final d'opération, INRAP, 297 p. (vol. 1), 289 pl. (vol. 2). 